MANFRED BOGEN

ROLAND KUCK

JENS SCHRÖTER (HRSG.)

\title{
Virtuelle Welten \\ als Basistechnologie für Kunst und Kultur?
}

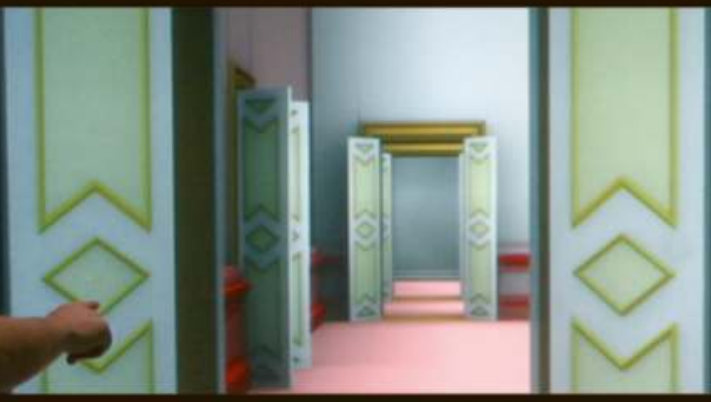

Eine Bestandsaufnahme 
Manfred Bogen, Roland Kuck, Jens Schröter (Hrsg.)

Virtuelle Welten als Basistechnologie für Kunst und Kultur? 
Die Reihe »Medienumbrüche« wird herausgegeben von Peter Gendolla. 
Manfred Bogen, Roland Kuck, Jens Schröter (Hrsg.)

Virtuelle Welten

als Basistechnologie für Kunst und Kultur?

Eine Bestandsaufnahme 
Diese Arbeit ist im Kulturwissenschaftlichen Forschungskolleg 615 der Universität Siegen entstanden und wurde auf seine Veranlassung unter Verwendung der von der Deutschen Forschungsgemeinschaft zur Verfügung gestellten Mittel gedruckt.

\section{Bibliografische Information der Deutschen Nationalbibliothek}

Die Deutsche Nationalbibliothek verzeichnet diese Publikation in der Deutschen Nationalbibliografie; detaillierte bibliografische Daten sind im Internet über http://dnb.d-nb.de abrufbar.

\section{(C) 2009 transcript Verlag, Bielefeld}

\section{(9) $\Theta \Theta \Theta$}

This work is licensed under a Creative Commons Attribution-NonCommercial-NoDerivatives 3.0 License.

Umschlaggestaltung: Kordula Röckenhaus, Bielefeld Umschlagabbildung: Das Titelbild zeigt Petros Kapakos in der virtuellen Galleria Borghese (dargestellt in dem TwoView-Display des Fraunhofer IAIS).

Druck: Majuskel Medienproduktion GmbH, Wetzlar

ISBN 978-3-8376-106I-I

Gedruckt auf alterungsbeständigem Papier mit chlorfrei gebleichtem Zellstoff.

Besuchen Sie uns im Internet: http://www.transcript-verlag.de

Bitte fordern Sie unser Gesamtverzeichnis und andere Broschüren an unter: info@transcript-verlag.de 


\section{Inhalt}

Gundolf Winter

Virtualität oder zur Inszenierung von Raumbildlichkeit

in der italienischen Wandmalerei

Jens Schröter

Die Ästhetik der virtuellen Welt

Überlegungen mit Niklas Luhmann und Jeffrey Shaw 25

Steffi Beckhaus

Zwischen real und digital: Intuitive, reichhaltige und

freudvolle Schnittstellen 37

Mario Doulis/Doris Agotai/Hans Peter Wyss

Spatial Interface

Wahrnehmungsfelder und Gestaltungsansätze im Virtuellen Raum 55

Christian Spies

Zwischen Kabinett und Second Life

Bildräume und Bilderräume 65

Markus Wacker/Matthias Lehmann/Ralph Stelzer

MySpace - Virtuelle Realitäten in dreidimensionalen Räumen 87

Tim Jansen/Roland Kuck

Interaktion in virtuellen Welten 99

Cornelius Weidner/Rolf Kruse

Die Begehung virtueller Realitäten

Severin Todt/Christof Rezk-Salama/Andreas Kolb

Virtuelle Rekonstruktion und interaktive Exploration der

Schlossanlage Dillenburg

Roland Kuck/Matthias Krauß/Gerold Wesche

Prozedurale Modelle 139

Autoren 



\section{Gundolf Winter}

\section{Virtualität oder zur Inszenierung von Raumbildlichkeit in der italienischen Wandmalerei}

Virtuelle Welten hat es immer schon gegeben, sind keine Erfindung des späten zwanzigsten und einundzwanzigsten Jahrhunderts, auch wenn es so scheinen mag, sondern sind ganz konkret die Mitrealität einer jeden künstlerischmalerischen Betätigung und solcherart bereits im Paläolithikum zu finden. ${ }^{1}$ Allerdings meint man heute mit virtuellen Welten etwas Anderes, Spezifisches, eben nicht nur allgemein fiktive Welten, sondern gerade solche, die mit unserer konkreten Wirklichkeit nahezu identisch scheinen, das heißt in ihrer Erscheinungsweise verwechselbar, die doch vom Faktischen her nichts weiter sind als Projektionen.

Aber auch für diese ,unsere‘ virtuellen Welten gibt es malereitechnisch gesehen Vor- und Frühgeschichten, von denen ich die frühneuzeitliche auswähle, da deren Basistechnologie eine weitgehend augentäuschende Erfahrungswirklichkeit veranlasste, die solcherart vielleicht Hinweise auch für aktuelle Überlegungen zu Prinzipien der Virtualität liefern könnte; und dies vielleicht um so mehr, als schon in der italienischen Wandmalerei des 15. Jahrhunderts nicht nur Bildräume zum Sehen entwickelt, sondern Raumbilder zum Gehen entworfen wurden Raumbilder, die den Betrachter in eine andere, neue, scheinhafte oder virtuelle Welt entführen, indem sie ihn mobilisieren, zum Gehen motivieren. ${ }^{2}$ Diese Bewegung, das Sich-Bewegen in der virtuellen Welt, d.h. in den Raumbildern, meint eine Mobilisierung des Betrachters, die vom Konzept (vielleicht auch von der Ideologie) her beim (traditionellen) Bildraum gerade nicht vorgegeben war. Zumindest war sie bei jenem klassisch geometrisch-projektiven Verfahren, das noch heute von vielen als Vorläufer der technischen Raumsimulationen verstanden wird, nicht vorgesehen: Bei der klassischen Perspektivkonstruktion ${ }^{3}$.

1 Zumindest dann, wenn man annimmt, dass virtuell als Eigenschaft einer Sache verstanden werden kann, die nicht in der Form existiert, in der sie zu wirken scheint, aber in ihrem Wesen und ihrer Wirkung einer real existierenden Sache entspricht. Solcherart wäre z.B. die Tierwelt von „Lascaux“ eine virtuelle.

2 So ist für die italienische Malerei der frühen Neuzeit aber auch sonst nicht allein das Tafelbild für Raumbildlichkeit verantwortlich, sondern in gleicher Weise die Wandund Freskomalerei - wie ja das erste, zentralperspektivisch exakt konstruierte Bild der Neuzeit ein Wandgemälde ist, Masaccios „Trinitätsfresko“; zum Trinitätsfresko siehe Huber: Das Trinitätsfresko von Masaccio und Filippo Brunelleschi in S. Maria Novella.

3 Ideologisch erweist sich die Perspektive auch als Symbol für eine strikt hierarchisch geordnete Gesellschaft: Man unterwirft perspektivisch sich die Welt, die auf den Augenpunkt hin ausgelegt ist und verfügt so über eine stabile Ordnung qua Rationalität; vgl. z.B. Arnheim: Kunst und Sehen, S. 287; siehe auch Damisch: Origine de la perspective; Jay: Downcast Eyes. 
Besonders deutlich wird das in der sog. „,novella di grasso“, einer Anekdote aus dem Leben Filippo Brunelleschis, dem Baumeister der Domkuppel in Florenz und „Erfinder“ der Perspektivkonstruktion. ${ }^{4}$ Normalerweise wird diese Anekdote zur Belustigung erzählt, um Brunelleschi als einen cleveren Kopf, guten Rechner und witzigen Zeitgenossen zu profilieren; mir scheint jedoch, dass sich darin auch etwas Bedenkliches, nämlich das Perfide der Perspektivkonstruktion und ihrer systematischen Ausrichtung auf einen - einzigen - Standpunkt hin ausdrückt. Der Erzählung zufolge hat Brunellschi den Florentiner Tischler Manetto Ammannatini, genannt „il grasso“, als Dummkopf vorgeführt, indem er / Brunelleschi - systematisch - alle Bekannten des Tischlers dafür gewann, den Tischler als einen Anderen auszugeben, was solange durchgehalten wurde, bis der Tischler schließlich selbst glaubte, ein Anderer / der Andere zu sein und - als Höhepunkt der Posse - sogar für dessen Trinkschulden aufkam.

Von dieser Anekdote (zur Charakterisierung Brunelleschis) kann man manches auf Brunelleschis wichtigste Erfindung, die Perspektivkonstruktion des Bildraums, übertragen. ${ }^{5}$ Denn wie bei der Geschichte vom fetten Tischler wird bei der klassischen Perspektivkonstruktion scheinbar Unmögliches möglich bzw. wirklich gemacht gerade dadurch, dass alles und Alle auf einen ganz bestimmten Standpunkt, einen einzigen Standort hin ausgerichtet werden; ${ }^{6}$. Man kann offenbar alles erreichen, wenn alles von diesem Standpunkt und nach diesem Standpunkt hin geordnet ist, selbst einen Wechsel der Identität. Die Identität des fetten Tischlers wurde in eine andere überführt aufgrund einer angenommenen Vorgabe, eines Standpunktes, die bzw. den Alle einhielten, so dass selbst der Betroffene sich der damit verbundenen Wirkung als Wirklichkeit nicht entziehen konnte. In ähnlicher Weise vermeint man bei einem perspektivisch konstruierten Bild - das auf den einen und einzigen Standpunkt hin berechnet ist - in einen Raum zu schauen, glaubt jene wie durch ein Fenster gesehene Wirklichkeit als die eigentliche Wirklichkeit wahrzunehmen, die doch nur bildliche Täuschung, nur die Wirkungsweise des räumlich Wirklichen wiedergibt, den ,actual fact“, nicht aber das Faktische, den ,factual fact“" 7

4 Berichtet wird die Anekdote von Manetti: Vita di Filippo Brunelleschi, dem ersten Brunelleschi-Biographen, der in der „Lebensbeschreibung“ den Schlüssel zum Verständnis der Anekdote anbietet; hier zitiert in der Übersetzung von Floerke: Die fünfundsiebzig italienischen Künstlernovellen der Renaissance, S. $141 \mathrm{ff}$.

5 Zu Brunelleschis „Perspektivtafeln“ siehe Pizzigoni: Filippo Brunelleschi.

6 Zur Perspektivkonstruktion und ihrer historischen, geistesgeschichtlichen und symptomatischen Bedeutung siehe Panofsky: Die Perspektive als „symbolische Form“ siehe auch Boehm: Studien zur Perspektivität, Philosophie und Kunst in der frühen Neuzeit; und neuerdings Belting: Florenz und Bagdad.

7 Zur Begrifflichkeit siehe Albers: Interaction of Color, S. 117f. 


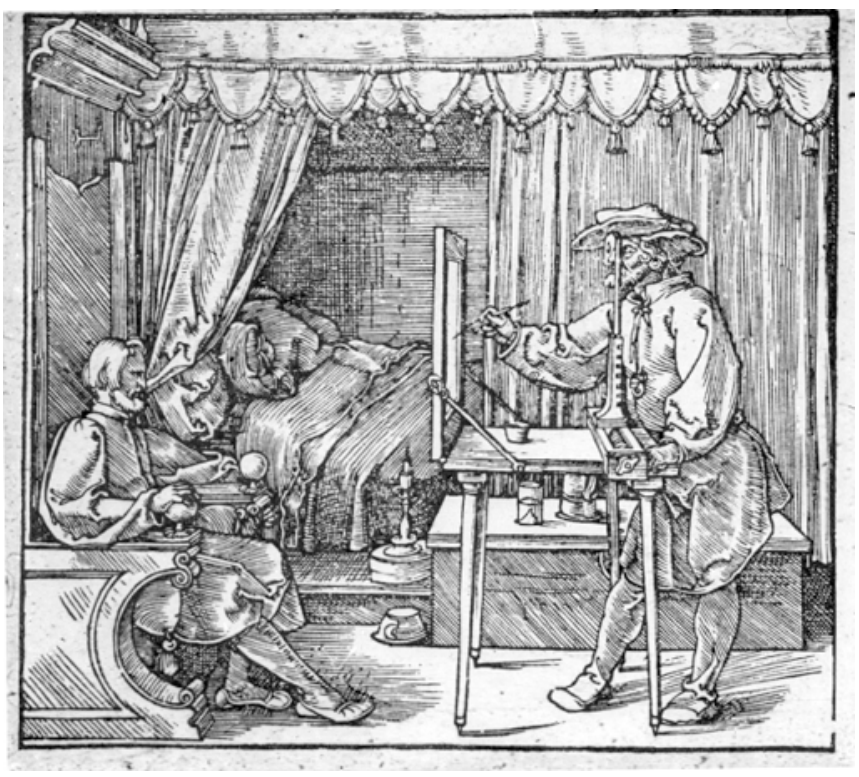

Abbildung 1: Albrecht Dürer, Der Zeichner des sitzenden Mannes, Holzschnitt, aus: Unterweisung der Messung

Was dieser zwanghaften Positionierung des - immobilen - Betrachters qua Perspektivkonstruktion konkret zugrunde liegt, kann vielleicht Dürers „Reproduktionsmaschine“ (Reproduktion der Wirklichkeit qua Perspektive) deutlich machen, die er seinem Zeichner in der „Unterweisung der Messung“"von 1525 an die Hand gegeben hat $^{8}$ : Dürers Graphik zeigt zunächst einen Innenraum; im Hintergrund befindet sich eine Lagerstätte, links eine männliche Figur in einem Thronsessel, die von einem Zeichner rechts - um in Dürers Worten zu reden gerade „abkonterfeit“ wird. Zur Erleichterung dieses Abkonterfeiens hat Dürer dem Zeichner eine Maschine zur Verfügung gestellt, welche drei wesentliche Voraussetzungen für die Malerei als Reproduktion von Wirklichkeit in Technik umsetzt. ${ }^{9}$ (Abb. 1 u. 2)

Der Zeichner steht an einem Tischchen, auf dem an der vorderen Kante in aufrechter Stellung eine Bildfläche angebracht ist, die aus einem Holzrahmen mit durchsichtigem Malgrund besteht. Sie vertritt das „Fenster“, durch das Welt gesehen wird und in welcher die sich in ihr abzeichnenden Formen der

8 Dürer: Unterweisung der Messung, Abb. S. 64, Text S. 62.

9 Jedenfalls ist an eine Wiedergabe gedacht, die im einzelnen sicher genauer und weniger idealisierend als Albertis Fensterblick ausfallen dürfte, auch wenn Dürer diese (künstlerische) Ansicht der Gegenstände aus der Natur heraus reißen möchte; siehe Dürer: Schriftlicher Nachlass, S. 295. 


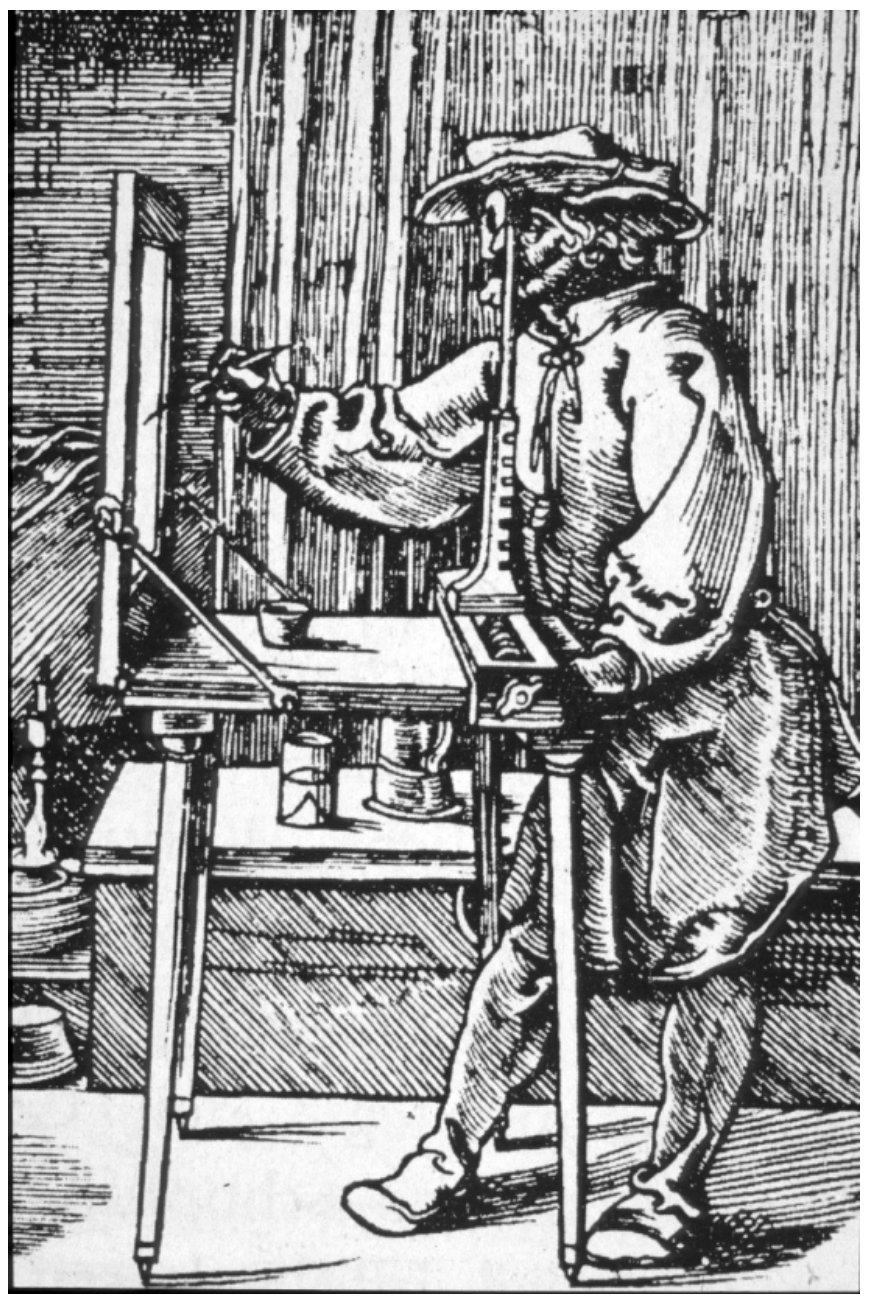

Abbildung 2: Albrecht Dürer, Detail 
zu sehenden Gegenstände, nachgezeichnet werden. An der gegenüberliegenden Kante des Tischchens ist ein bewegliches Querholz vermittels einer Zwinge festgeschraubt, auf dem wiederum ein aufrecht stehender Holzstab befestigt ist, der am oberen Ende eine Scheibe trägt, in welche ein Loch gebohrt ist, durch das der Zeichner mit einem Auge auf bzw. durch die Bildscheibe blickt. Die Maschine macht durch diese Technik deutlich, welche Voraussetzungen das Sehen erfüllen muss, damit Malen als Reproduktion von Wirklichkeit erscheinen und damit das Bild als ein auf Welt geöffnetes Fenster verstanden werden kann.

Es darf erstens nur mit einem einzigen und unbewegten Auge gesehen werden - dem entspricht die Vorrichtung der Scheibe mit dem Loch am aufrechten Stab rechts; dann darf nur in einem fixierten Abstand zur Bildfläche gesehen werden - dem entspricht das festgestellte Querholz; und schließlich darf nur so gesehen werden, dass die Sehpyramide (jene geometrische Figur, die sich ergibt, wenn verschiedene Punkte der zu betrachtenden Gegenstände mit dem Auge des Betrachters durch Linien, den sogenannten Sehstrahlen, verbunden werden) durch die Fläche der Glasscheibe gerade durchschnitten wird - dem entspricht die aufrechte Bild- oder Fensterfläche am linken Ende des Tischchens. Gemäß diesen Vorraussetzungen - so die Konstruktion - werde das Bild auf der Glasscheibe mit jenem übereinstimmen, das beim Sehen auf der Netzhaut des Auges entsteht. ${ }^{10}$ Übereinstimmen würde damit die Erscheinungsweise der Gegenstände in der zweidimensionalen Fläche mit ihrer Erscheinungsweise im dreidimensionalen Raum, da sie jeweils, das zweidimensionale Bild wie das Vorbild im dreidimensionalen Raum, unter den gleichen Sehbedingungen erfahren würden. Damit wäre dann auch die Täuschung perfekt, und wir können - so scheint es - in eine neue, andere Wirklichkeit eintreten.

Das erste in dieser Art neuzeitliche, also zentralperspektivisch exakt durchgeführte Bild der Kunstgeschichte - und hiermit komme ich zur italienischen Wandmalerei - zeigt die Bildwirklichkeit nicht nur ,wie durch ein Fenster“, also nicht wie durch ein Loch in der Wand, durch das man eine andere Wirklichkeit erblickt, sondern wie durch ein Portal; denn es öffnet sich ein ganzer Kapellenraum, ein Anschauungsraum, der den konkreten Betrachterraum erweitert, in den man auch vermeint, hineingehen zu können ${ }^{11}$ (Abb. 3); zumindest lädt dazu der Altartisch ein und weitere Elemente, auch wenn man dann der Aufforderung instinktiv doch nicht nachkommt. Nicht wegen der Gefahr, vor die Wand zu laufen, sondern weil die Kapelle bereits durch die Trinität mit Maria und Johannes besetzt scheint. Dieses erste, zentralperspektivisch korrekt kon-

10 Dies wäre zumindest der Effekt, wenn man Albertis Fenster-Metapher ernst nehmen würde. Zum „geöffneten Fenster“ als Metapher für das neuzeitliche Bild siehe Winter: Durchblick oder Vision.

11 Das betrifft nicht nur die gemalte Kapelle, sondern vor allem auch den realen Altar, der den Übergang von Faktischem und Fiktivem verschleiert bzw. fließend macht. 


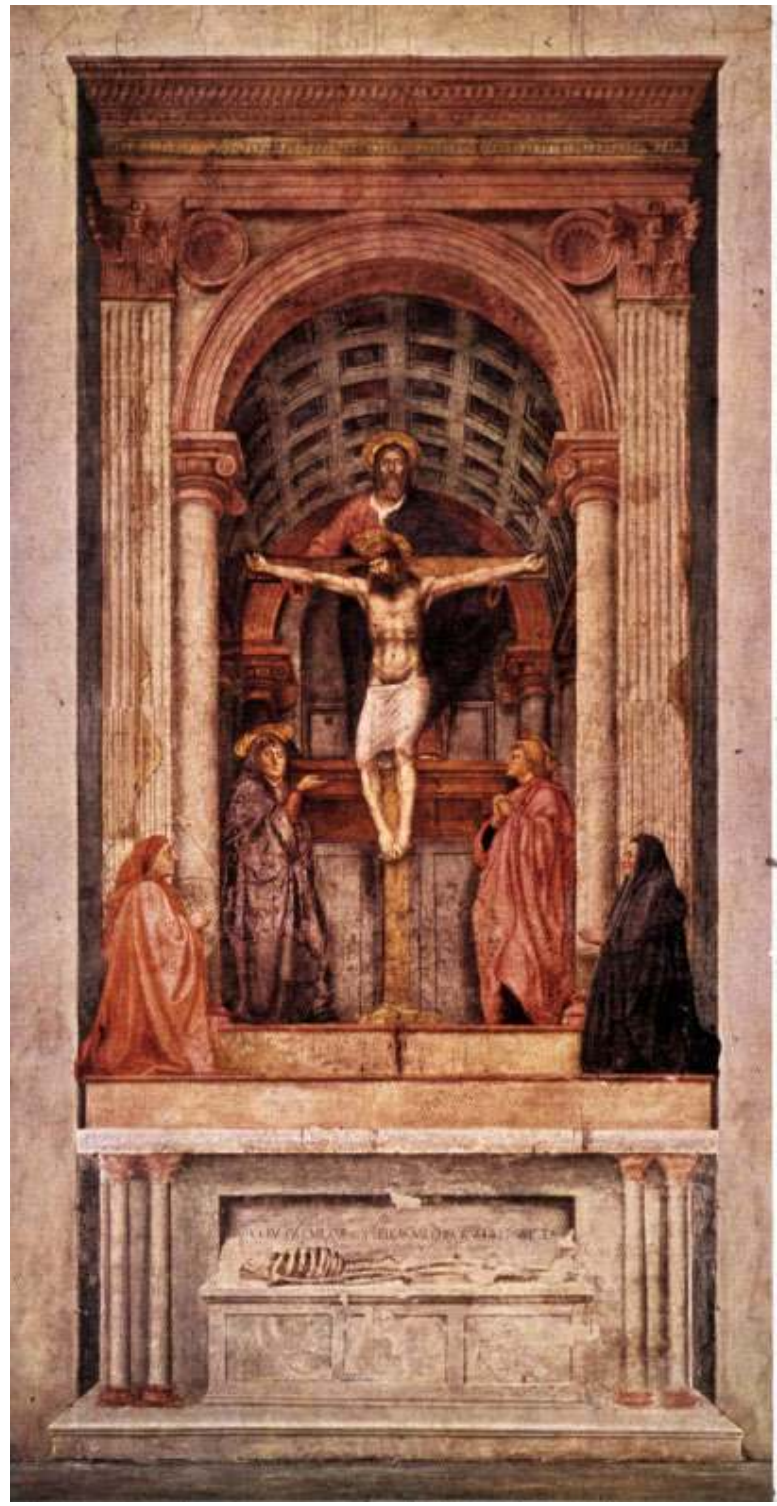

Abbildung 3: Masaccio, Trinität, Fresko, S. Maria Novella, Florenz 
struierte Bild der Kunstgeschichte, das Trinitätsfresko in Santa Maria Novella in Florenz, malte Masaccio 1428 in vierundzwanzig Tagwerken ${ }^{12}$. Aber Masaccio malte nicht ohne Hilfe bzw. Unterstützung. Seinem Freund Brunelleschi, der die zentralperspektivische Konstruktion zum ersten Mal zwischen 1415 und 1420 in Florenz demonstriert hatte, werden hierbei wesentliche Anregungen und Motive zugeschrieben. So verrät nicht nur die gemalte Architektur - Brunelleschi war vor allem Architekt -, sondern auch ihre Ausbildung als Kapellenarchitektur seine Handschrift, wenn er nicht überhaupt für die Raumerweiterung verantwortlich zu machen ist. ${ }^{13}$

In dieser Abbildung sehen wir Masacccios Fresko - zumindest habe ich eine Rekonstruktion versucht - in einem seiner ursprünglichen Umgebung nahe kommenden Kontext ${ }^{14}$ (Abb. 4), wodurch deutlich werden soll, um was es bei der perspektivischen Raumerweiterung zugleich immer auch und von Anfang an ging, nämlich um die perfekte Imitation von Wirklichkeit, nicht nur um eine neue oder erweiterte Wirklichkeit, sondern um virtuose Vortäuschungen, um die Zauberkünste des Spiegels. Und - es ging um Macht, um die Beherrschung von Welt (oder Welten), d.h. um ihre Feststellung von einem so und nicht anders, und zwar von mir gesetzten Standpunkt aus. ${ }^{15}$ Von diesem her muss alles erfahrbar, logisch und klar geordnet sein, dann herrsche ich über Raum und Zeit. Zumindest für jenen kurzen Augen-Blick, in dem ich selbst diesen Standpunkt einnehme, mich ihm unterwerfe, ein immobiler Betrachter werde. Mit der Konsequenz, dass die neue Machtvollkommenheit, die Herrschaft über Raum und Zeit und - wie wir beim fetten Tischler gehört haben - Identität erkauft werden muss mit einem kaum zu überbietenden Zwang, der meinen Standpunkt, meine Blickweise und die damit verbundene Systematisierung des Sehens betrifft.

Gleichwohl hat schon Masaccio in der künstlerischen Anverwandlung der Perspektiv-Konstruktion eigentlich keinen immobilen Betrachter für seine Darstellung vorgesehen, sind wir jedenfalls nicht durch die Konstruktion der Raumerweiterung an eine Stelle gebannt, sondern gibt es deutlich Toleranzen im Hinblick auf die Mobilität des Betrachters. Dies hat wiederum mit der

$12 \mathrm{Zu}$ Masaccio und dem Trinitätsfresko siehe Hertlein: Masaccios Trinität; Kemp: Masaccios Trinità im Kontext; Johannides: Masaccio und Masolino, S. $177 \mathrm{ff}$.

13 Andererseits scheint die Ausstattung der Kapelle vom Werk eines anderen Freundes angeregt zu sein, von Donatellos Grabmal Papst Johannes' XXIII im Baptisterium von Florenz; zu Donatellos Grabmal siehe Poeschke: Die Skulptur der Renaissance in Italien, S. 97f.

14 Die „Umgebung“ ist natürlich nicht ursprünglich, denn sie ist die von S. Croce und nicht von S. Maria Novella. Aber es ging um die ursprüngliche Umgebung mit Grabkapellen, Epitaphien und Altären, die so ehemals auch in S. Maria Novella zu finden war, jetzt aber eher in S. Croce anzutreffen ist.

15 Siehe Panofsky: Die Perspektive als „symbolische Form“, S. 123 f. 


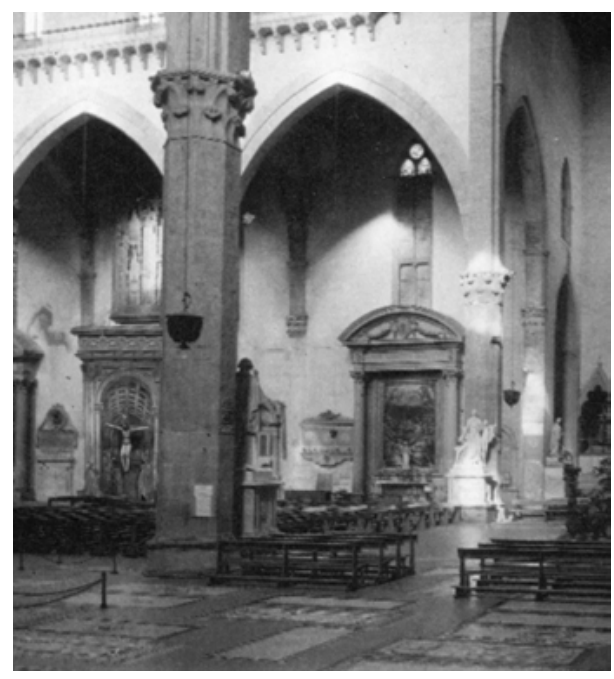

Abbildung 4: Trinitätsfresko von Masaccio nach S. Croce, Florenz, verlagert. (Fotomontage, Winter)

Art der Raumkonstruktion zu tun, die Masaccio untersichtig angelegt hat, so dass wir die Fußpunkte der Kapelle, nämlich die entscheidenden Schnittstellen zwischen Fußboden und aufgehenden Wänden, nicht sehen können. Mit diesem ,Trick' sehen wir zwar in einen tatsächlichen, nämlich exakt konstruierten (Kapellen-)Raum, aber der konkrete Bezugspunkt zur gemalten Architektur des ,realen' (Kirchen-)Raums ist nicht sichtbar, sondern nur erschließbar und öffnet von daher die Möglichkeit von Toleranzräumlichkeit.

Dies mag im vorliegenden Fall einer lediglich seitlichen Raumerweiterung vielleicht nebensächlich erscheinen, hat aber erhebliche Konsequenzen für Rauminszenierungen, wie sie im Anschluss an Mantegnas „Camera degli Sposi“ im profanen und sakralen Rahmen verstärkt in Erscheinung treten. ${ }^{16}$ Denn dort befindet man sich dann innerhalb, in Mitten des virtualisierten Raumes. Dieser ist nicht lediglich eine partielle Erweiterung des konkreten und damit Bildraum, sondern vollständig virtualisiert und damit Raumbild. Seit Mantegna haben wir es folglich zunehmend mit Raumbildern zu tun, in denen sich der Betrachter konkret aufhält und dadurch auch zunehmend in ein Dilemma gerät. Denn entweder folgt er der vorgegebenen Raumkonstruktion - also der Perspektive und ihrem Fluchpunktrigorismus - und steht immobil, d.h. er verhält sich zum Raum (wie bei einem Bild), nicht im Raum. Oder er folgt den Gegebenheiten des

$16 \mathrm{Zu}$ Mantegna und der „Camera degli Sposi“, die zunächst und bis ins 17. Jahrhundert „Camera picta“ hieß, siehe De Nicolò Salmazo: Andrea Mantegna, S. 161ff.; Cordaro: La camera degli sposi. 
- wenn auch in seiner Ausdehnung, Anordnung usw. - vorgetäuschten Raums, dann möchte er sich - wie bei jedem konkreten Raum - bewegen. Was aber wiederum aufgrund der Raumkonstruktion nicht möglich erscheint, es sei denn, sein Bewegungswunsch wäre von der Raumkonstruktion des virtuellen Raums mitberücksichtigt worden, die dann aber keine Ein-Fluchtpunktperspektive sein könnte, was wiederum Schwierigkeiten bereiten dürfte, da wir es ja bei der augentäuschenden Raummalerei immer mit vermischten Raumwirklichkeiten zu tun haben. ${ }^{17}$

Mantegnas „Camera degli Sposi““ von 1474 zeigt einen solch“virtuellen Raum (Abb. 5), in dem wir uns konkret ,mittendrin` befinden. Dabei haben wir es hier nicht nur mit räumlichen Erweiterungen nach allen Seiten, sondern auch nach oben hin zu tun, sehen wir hier (zum ersten Mal) in extremer Untersicht Personen, Putten und einen bedenklich balancierten Blumenkübel gegen den Himmel (Abb. 6), in den wir hinaufschauen, während die Personen und Putten auf uns herunterschauen, um so die Dimensionen des Oben und Unten zu aktualisieren. Auch die Weitung zu den Seiten hin zeigt sich verstärkt in Raumtiefe und Raumtäuschung, da sich die Wände auf verschiedene Raumschichten öffnen, wobei die Wandarchitektur durchweg, bis auf wenige faktisch-konkrete, also steinerne Architekturelemente (Konsolen) gemalt ist: Pilaster, Marmorsockel und Deckenkonstruktion sind Schein, Augentäuschung. Aber dieser Bildzauber ist doch - und deutlicher noch als bei Masaccios Trinitätsfresko - nicht auf einen Augenpunkt hin festgelegt, auch an der Decke nicht, sondern polyfokal organisiert, nämlich so, dass ein mobiler Betrachter vorausgesetzt wird. ${ }^{18}$

Selbst wenn man - um Analogien zu den heutigen virtuellen Räumen aufzumachen - dann nicht wirklich von einem im Raumbild programmierten Betrachter sprechen kann, weist Mantegna doch unterschiedlich „angemessene“ Standpunkte für die verschiedenen Seiten und die Decke aus, muss sich der Betrachter bewegen, gleichsam mitspielen, wenn er an der „Begegnung“ teilhaben oder sich in den „Hofstaat“ einreihen will; er muss sich bewegen, Einzelnes in den Blick nehmen, und kann dies auch, ohne dabei die Gesamträumlichkeit, den Gesamtraum zu verlieren. So findet sich das Ganze im selbständig Einzelnen

17 Dieser Übergang ist für die traditionellen Scheinwelten typisch, die sich aus dem Realen entwickeln und mit diesem auch immer Verbindung halten. Dies ist in den neuen virtuellen Welten anders: Die Immersion möchte umfassend sein, was nur unter Ausschluss des Realen möglich sein dürfte. Neuerdings gibt es Bestrebungen, im Rahmen der ,,augmented reality“ Realität in die Virtualität miteinzubeziehen; zur „augmented reality“ siehe Bimber/Raskar: Spatial Augmented Reality.

18 Was der Illusion des Eintauchens in eine andere, fiktive Welt freilich keinen Abbruch tut; es kommt nicht zu ,Brüchen', weil die Orientierung am dekorierten Ganzen nie verloren geht; dies im Unterschied zu Giovanni Paccagnini, der nur einen einzigen Standpunkt annimmt: Paccagnini: La camera degli sposi. 


\section{Gundolf Winter | Virtualität}

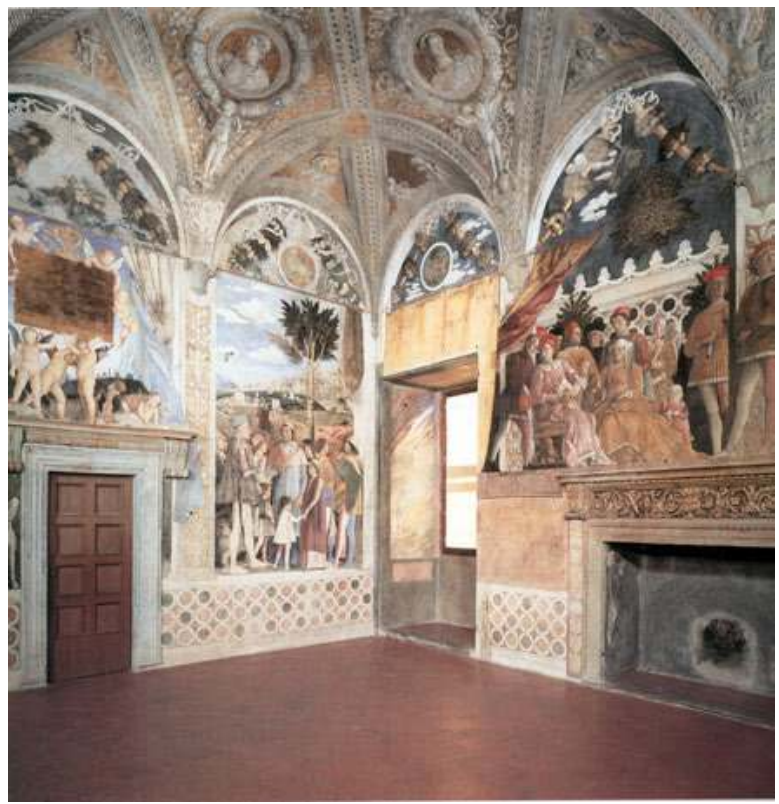

Abbildung 5: Andrea Mantegna, Camera degli Sposi, Fresko, Castello di S. Giorgio, Mantua

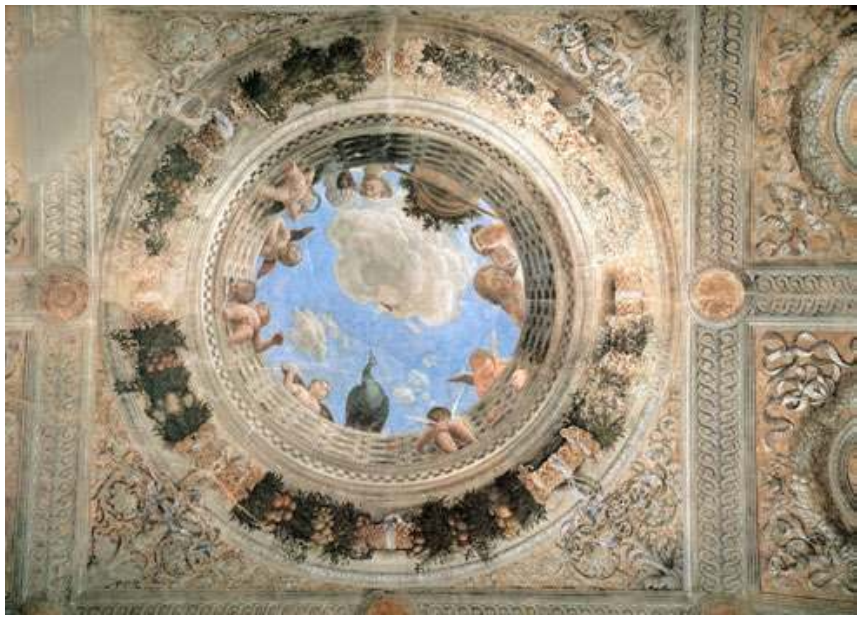

Abbildung 6: Andrea Mantegna, Camera degli Sposi, Deckengemälde, Mittelfeld 
und auch einzeln Konzipierten mitpräsent, wie sich das selbständig Einzelne ebenso im Gesamten aufgehoben enthalten weiß. ${ }^{19}$ Der Betrachter kann sich seinen Weg suchen, aber er wird vom Ganzen begleitet, das zentral, von der Mitte her, optisch gesteuert wird. Der reale Raum ist malerisch gefasst und dabei neu geordnet, ausgerichtet (durch die gemalte Architektur) und auch weitergeführt dergestalt, dass hier eine virtuelle Umgebung geschaffen wurde, deren Neuartigkeit darin besteht, dass sie sich als die wirkliche auszugeben sucht, zumindest in der Weise, dass entscheidende Elemente der wirklichen Umgebung des Palastes als räumlich und zeitlich verstreute und mit jeweiligen Ansprüchen versehene als örtlich und aktuell fundierende unmittelbare raumbildliche Präsenz gewinnen.

Der malerisch erzeugte virtuelle Raum der „Camera degli Sposi“ mit seiner mehrdimensionalen Erweiterung wird kurz darauf von Coreggio dadurch gesprengt, dass er die Dimension der Höhe freisetzt, so dass wir den Himmel tatsächlich - auch in religiöser Dimension - offen sehen und das heißt: In eine andere Welt, eine andere, neue Räumlichkeit blicken. ${ }^{20}$ Verschiedene Welten öffnet uns auch Michelangelos „Sixtinadecke“, Welten, die sich an der gemalten Architektur ausrichten, die sich wohl auf die faktische der Kapellenarchitektur beruft, diese aber nicht fortsetzt, sondern frei und neuartig weitermalt. ${ }^{21}$ Die gemalte Architektur gibt dann den Rahmen für verschiedene Ausblicke, Anblicke und Einblicke, die - solcherart differenziert - gleichwohl eines gemeinsam haben: Sie sind gemalt und als diese gemalten Anblicke, Ausblicke und Einblicke konkret Bilder. Sie dienen als illusionistische Erweiterungen des Raumes, wobei aber weniger eine wie immer zu denkende perspektivische Raumdarstellung für die Bildräume bestimmend ist, sondern die Dynamik der in ihnen erscheinenden Körper; dies auch bei den Einblicken, also den eigengerahmten, eigenperspektivischen Bildern der Decke. ${ }^{22}$

Für Michelangelo ist folglich die Bewegung entscheidend: Hatte Coreggio, bildlich inszeniert, die Bewegung nach oben angestoßen, so haben wir hier, ebenfalls bildlich inszeniert, Bewegungen in alle Richtungen hin orientiert, ge-

19 Was dem Schönheitsideal der Renaissance nach Alberti entspricht; siehe Alberti: Zehn Bücher über die Baukunst, S. 492: „Die Schönheit ist eine Art Übereinstimmung und ein Zusammenklang der Teile zu einem Ganzen, das nach einer bestimmten Zahl, einer besonderen Beziehung und Anordnung ausgeführt wurde, wie es das Ebenmaß, das heißt das vollkommenste und oberste Naturgesetz fordert".

20 Etwa im Kuppelfresko der Kathedrale von Parma; zu Corregio siehe Ekserdjian: Corregio, S. $241 \mathrm{ff}$.

21 Zu Michelangelos Sixtina-Decke siehe De Tolnay: The sistine ceiling; Kuhn: Michelangelo. Die sixtinische Decke, S. 45ff.; De Vecchi: Die sixtinische Kapelle, S. 82ff.

22 Dies ist aber für die gesamte italienische Wand- und Deckenmalerei zu sagen, dass sie sich in ihrer räumlichen Illusionsbildung selten auf die reine Raumkonstruktion verlässt, sondern dabei immer auch und bei Michelangelo in entschiedener Weise auf den menschlichen Körper baut. 
rahmt zwar, aber nicht synchronisiert: Dieses Gegeneinander verlangt wieder den mobilen Betrachter, der sich zu verschiedenen Stellen zu begeben hat, von denen er dann jeweils die Ausblicke, Einblicke und Anblicke am besten sehen kann; solcherart wird Bewegung programmiert, allerdings nur die Bewegung; ihre Einbindung in ein Gesamt wird nicht vorgenommen: Kunsthistorisch gesprochen ein bereits manieristisches Konzept. Entsprechend erscheint das iterative Element der Deckenfresken stärker als der einheitliche Architekturrahmen, findet der Betrachter keinen wirklichen Stand- oder Ruhepunkt für die Erfahrung des Gesamten. ${ }^{23}$ Das Gesamte muss aus den einzelnen Blickpunkten und den mit ihnen verbundenen Seherfahrungen zusammengesetzt, das Übergreifende in der Vorstellung erzeugt werden: Im An-, Aus- oder Einblick ist es nicht zu haben. Der mobile Betrachter stellt sich so einer eminent gedanklichen Herausforderung.

Was radikal im Unterschied zu Andrea Pozzos Arbeiten in S. Ignazio zu sehen ist, die im Rahmen einer Illusionsmalerei, der Quadraturmalerei, nicht nur eine räumlich organisierte Einheitssetzung von unterschiedlichen Bildformen und Bildprogrammen, sondern zugleich eine Einheitssetzung von Immanenz und Transzendenz unter der Gesetzmäßigkeit des Empirischen zu erreichen suchen $^{24}$ (Abb. 7 u. 8). Damit zielt Pozzo auf eine virtuelle Gesamtschau, auf eine universelle Einheitssetzung als Ereignis im Raum der Kirche. Um die Himmelssphäre unter den Bedingungen des Empirischen zu fassen, war es notwendig, den konkreten Ordnungsrahmen des Empirischen, hier die Architektur des Sakralraums, auch für den Himmel verbindlich zu erklären. Infolge dessen folgt Pozzos Malerei den konkreten Vorgaben des Raumes, dessen Ordnungsstruktur die Architektur besorgt. Wir haben es folglich primär mit Architekturmalerei zu tun, welche die gebaute Architektur weiterführt, illusionistisch in einer (gemalten) Attika und weiterführenden Architekturgliedern derart, dass es - wenn man an der richtigen Stelle steht, die freundlicherweise durch eine gelbe Marmorscheibe im Fußboden angegeben ist - den Anschein hat, als setze sich die Architektur der Kirche trotz Decke und faktischem Tonnengewölbe fort und gebe schließlich sogar den räumlichen Rahmen für die Erschließung des Jenseitigen bzw. des Göttlichen. ${ }^{25}$

23 Die Ruhelosigkeit und - damit verbunden - der Verlust der prästabilierten Harmonie der Renaissance macht sich hier gerade auch im Vergleich mit Mantegnas Raummalerei mit Nachdruck bemerkbar.

24 Zum Konzept seiner Decken- und Wandmalerei siehe Schöne: Zur Bedeutung der Schrägansicht für die Deckenmalerei des Barock; Kerber: Andrea Pozzo; Kerber: Anamorphose und Illusion im Werk Andrea Pozzos; Burda-Stengel: Andrea Pozzo und die Videokunst.

25 Eine so weitgehende, Übereinstimmung und das heißt Synthese von Real- und Idealbzw. Illusionsräumlichkeit hatte es bislang nicht gegeben. 


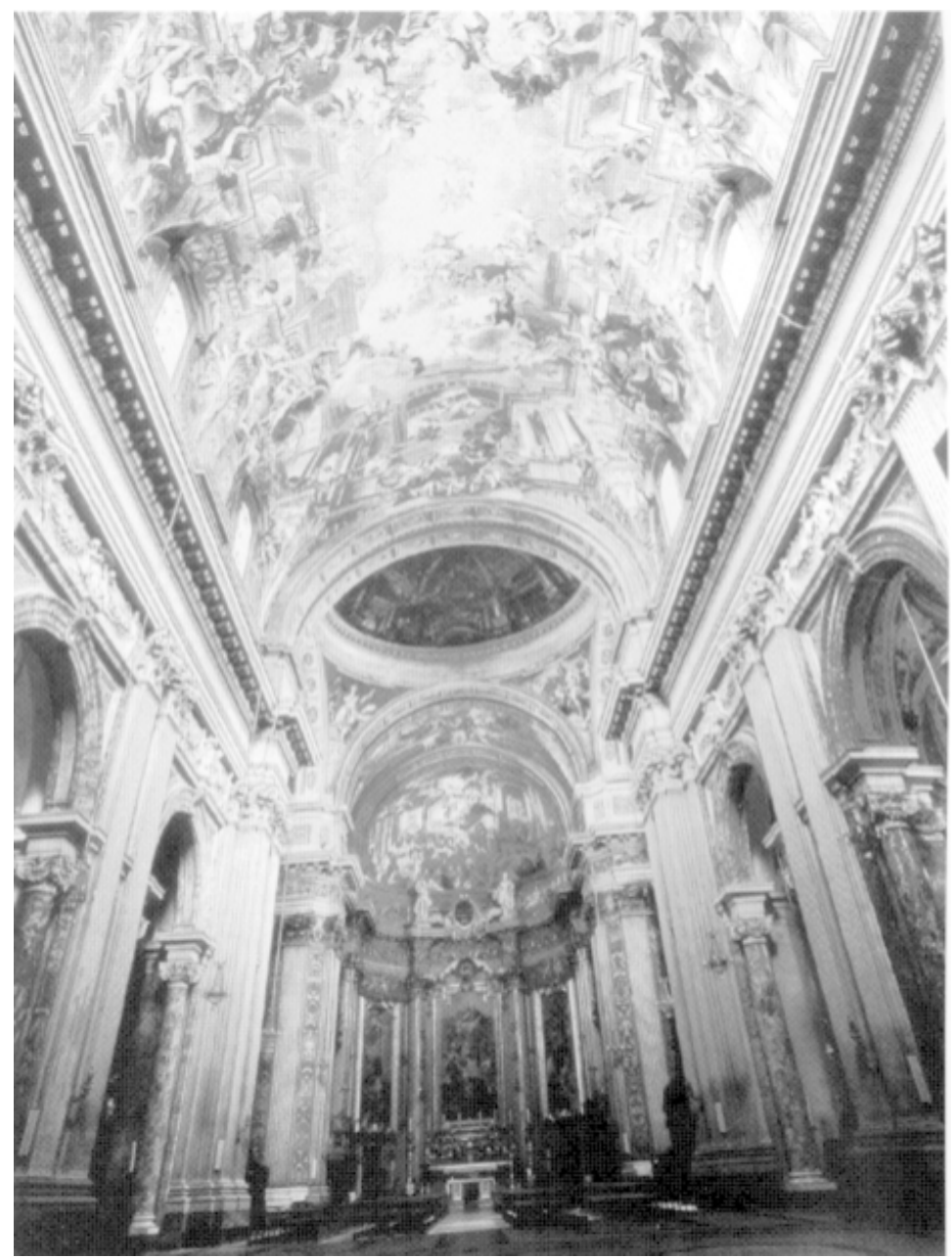

Abbildung 7: Andrea Pozzo, S. Ignazio, Rom, Deckenmalerei, (Blick zum Chor) 


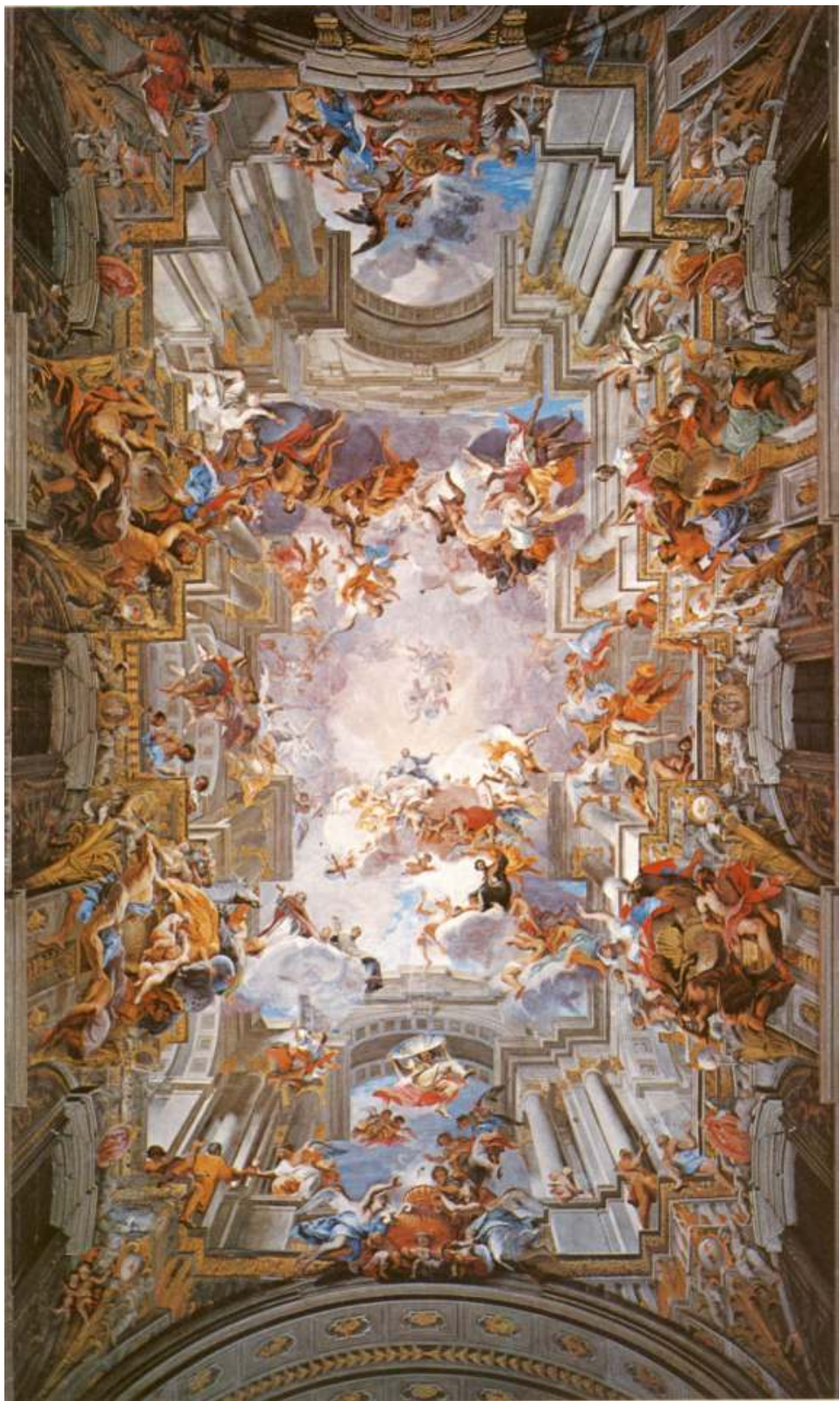

Abbildung 8: Andrea Pozzo, S. Ignazio, Rom, Deckenmalerei, (Langhausgewölbe) 
Freilich, diese so intensiv und unmittelbar erfahrbare Einheit von Immanenz und Transzendenz, und - damit verbunden - die größtmögliche Machtentfaltung des Blicks und folglich des Bildlichen wird erkauft mit der größtmöglichen Feststellung des Blicks bzw. des Betrachters. Pozzo richtet alles wieder auf einen einzigen Standpunkt aus, gibt nun tatsächlich sein Raumbild für diesen einzig möglichen Standort, verlangt erneut, wenn auch unter ganz neuen, barocken Bedingungen, den immobilen Betrachter. Nur wenn der Betrachter den Gegebenheiten der Perspektive rückhaltlos folgt, sich ihnen unterwirft, wird ihm die universelle Sicht zuteil, sieht er im Augenblick das Sich-Verbinden von Immanenz und Transzendenz, die universelle Einheit; konkret also dann, wenn der Mensch zum Auge verkommt, seine somatische Existenz verliert, wenn sich der Blick vom Körper trennt.

In der kunsthistorischen Auseinandersetzung mit den virtuellen Räumen hat Pozzos Deckenfresko eine besondere Bedeutung bekommen: An diesem äußerst suggestiven, bezeichnender Weise für eine Jesuitenkirche gemalten, bildräumlich organisierten Raumbild wurden Perspektiven und Paradigmen der virtuellen Realität diskutiert ${ }^{26}$. Dem Dilemma, dass wir es hier mit einem Raumbild zu tun haben, das gleichwohl - wie sonst allein der Bildraum der perspektivischen Konstruktion - den immobilen Betrachter verlangt, wollte, so scheint mir zumindest, Burda-Stengel in seiner Pozzo-Studie dadurch entkommen, dass er versuchte, den mobilen Betrachter wieder einzuführen, ${ }^{27}$ und zwar als einen, der den ,Betrug“ der Decke zunächst einsehen soll, bevor er dann - mit dem richtigen Bewusstsein ausgestattet - die Täuschung nach der Enttäuschung erleben darf. Eine etwas schwierige (jesuitische?) Konstruktion, der zufolge ein Raum zu 99\% aus gemalten Irrtümern besteht, die nur dazu dienen sollen, das 1\% einnehmende, vorgetäuschte Ergebnis tatsächlich als Täuschung zu erkennen.

Doch scheint das Ergebnis höchst aufschlussreich für einen Vergleich mit den „Künstlichen Umgebungen“, da in S. Ignazio sowohl die Exaktheit der Augentäuschung als auch die Bedingung für ihre Ermöglichung und damit das Dilemma der augentäuschenden künstlerischen Umgebungen ganz allgemein in aller Klarheit hervortreten: Pozzo inszeniert den Illusionsraum einer universellen Einheit, gibt diese perfekt künstliche Umgebung als künstlerische, die aber nur von einem Punkt aus zu erfahren ist. Entfernt man sich von diesem Punkt, wird der Einheitsraum zerstört, treten die Teile auseinander, bleibt der Deckenraum als falscher Bildraum auf der Strecke. Auch mit diesem Bild der Zerstörung sieht sich der immobile Betrachter in S. Ignazio, wenn er denn doch mobil wird, konfrontiert. $^{28}$

26 Vgl. Burda-Stengel: Andrea Pozzo und die Videokunst, S. 109ff. u. S. 131 f.

27 Siehe Burda-Stengel, a.a.O. S. 9 ff.

28 Aber er ist ja vom Konzept, der Quadraturmalerei, als immobiler Betrachter vorgesehen; alles andere wären moderne Missverständnisse, wie Kerber bündig darlegt: Kerber: Anamorphose und Illusion im Werk Andrea Pozzos, S. 199f. 
In den heutigen künstlichen Umgebungen geschieht im Prinzip das gleiche: Hier gilt freilich die Regel: Mit jedem Punkt der interaktiv geregelten neuen Raumansicht wird die alte, vorherige ausgeblendet, zerstört. Dies aber bleibt verborgen, ist nicht zu sehen, da jede neue Sichtweise absolut gesetzt wird, folglich Zerstörungen im rein Präsentischen der jeweiligen Raumpräsentationen nicht vorkommen können, da sie immer schon geschehen sind - wir werden an jeder Stelle zum „fetten Tischler“ gemacht. Bei Pozzo sehen wir die Zerstörung der künstlichen Umgebung, die bei ihm freilich passieren muss, weil sein Raumbild ein Hybrid darstellt, reale Architektur, der konkrete Kirchenraum, den Illusionsraum integriert, ja der alles umfassende Illusionsraum sich aus dem konkreten Kirchenraum allererst heraus entwickelt. Wobei dieser Bezug zur Kirche, zum Ort des Geschehens natürlich immer mitgemeint war. In den virtuellen Umgebungen unserer Tage würden wir an dieser Stelle - in einem 3x3 Meter dimensionierten Raum - vor die Wand laufen. Das Dilemma der virtuellen Realität, das Dilemma des Raumbildes, das den mobilen Betrachter ermöglicht, weil jede Stelle den Betrachter immobilisiert, wird so souverän überspielt. ${ }^{29}$

Neuere Überlegungen scheinen darauf abzuzielen, in den künstlichen Umgebungen auch diesen Zusammenhang mit der konkreten Umgebung abzubilden, also auch den Übergang oder die Gleichzeitigkeit von konkreter und simulierter Wirklichkeit herzustellen. ${ }^{30}$ Für die Wand- und Deckenmalerei immer schon vorgegebenes Thema wäre es interessant zu erfahren, wie mit jenen, im Medium der Malerei vorgestellten Problemen in den neuen Medien bzw. künstlichen Umgebungen ,technisch“ umgegangen werden kann.

\section{Literatur}

Albers, Josef: Interaction of Color. Grundlegung einer Didaktik des Sehens, Köln 1970.

Alberti, Leon Battista: Zehn Bücher über die Baukunst, übers. u. komm. v. Max Theuer, Wien und Leipzig 1912, Reprint Darmstadt 1975.

Arnheim, Rudolf: Kunst und Sehen. Eine Psychologie des schöpferischen Auges, Berlin-New York 1978.

Belting, Hans: Florenz und Bagdad. Eine westöstliche Geschichte des Blicks, München 2008.

29 Was zu einer immer nur fragmentarischen, ausschnitthaften Wirklichkeitsvorstellung führt.

30 Zumindest ist dies im Rahmen der ,augmented reality“ Projekte angestrebt; zu Projekten dieser Art siehe Haller/Billinghurst/Thomas: Emerging Technologies of Augmented Reality. 
Bimber, Oliver/Raskar, Ramesh: Spatial Augmented Reality: Merging Real and Virtual Worlds, Wellesley, Mass. 2005.

Boehm, Gottfried: Studien zur Perspektivität, Philosophie und Kunst in der frühen Neuzeit, Heidelberg 1969.

Burda-Stengel, Felix: Andrea Pozzo und die Videokunst, Berlin 2001.

Cordaro, Michele (Hrsg.): La camera degli sposi, Mailand 1992.

Damisch, Hubert: Origine de la perspective, Paris 1993.

De Nicolò Salmazo, Alberta: Andrea Mantegna, Köln 2004.

De Tolnay, Charles: The sistine ceiling, Princeton 1969.

De Vecchi, Pierluigi: Die sixtinische Kapelle, Freiburg-Basel-Wien 1996.

Dürer, Albrecht: Schriftlicher Nachlass, hrsg. v. Hans Rupprich, Band 3, Berlin 1969.

Dürer, Albrecht: Unterweisung der Messung, Neudr. der Ausg. von 1525, 2. Auflage. Nördlingen 1983.

Ekserdjian, David: Corregio, New Haven u. London 1997.

Floerke, Hans.: Die fünfundsiebzig italienischen Künstlernovellen der Renaissance, München und Leipzig 1913.

Haller, Michael/Billinghurst, Mark/Thomas, Bruce: Emerging Technologies of Augmented Reality: Interfaces and Design, Hershey 2007.

Hertlein, Edgar: Masaccios Trinität. Kunst, Geschichte und Politik der Frührenaissance in Florenz, Florenz 1979.

Huber, Florian: Das Trinitätsfresko von Masaccio und Filippo Brunelleschi in S. Maria Novella, München 1990.

Jay, Martin: Downcast Eyes, The denigration of vision in twentieth-century French thought, Berkeley 1993.

Johannides, Paul: Masaccio und Masolino. A complete catalogue, London 1993.

Kemp, Wolfgang: Masaccios Trinità im Kontext, in: Marburger Jahrbuch für Kunstwissenschaft, 1986, Nr. 21, S. 45ff.

Kerber, Bernhard: Andrea Pozzo, Berlin - New York 1971. 
Kerber, Bernhard: Anamorphose und Illusion im Werk Andrea Pozzos, in: Winter-Bilder, Festschrift für Gundolf Winter, Siegen 2003, S. $197 \mathrm{ff}$.

Kuhn, Rudolf: Michelangelo. Die sixtinische Decke, Berlin-New York 1986.

Manetti, Antonio Di Tuccio: Vita di Filippo Brunelleschi, preceduta da „La novella del Grasso“, Mailand 1976.

Paccagnini, Giovanni: La camera degli sposi, Mailand 1957.

Panofsky, Erwin: Die Perspektive als „symbolische Form“, in: Oberer, H./ Verheyen, E. (Hrsg.): Aufsätze zu Grundfragen der Kunstwissenschaft, Berlin 1974, S. $99 \mathrm{ff}$.

Pizzigoni, Attilo: Filippo Brunelleschi, Zürich und München 1991.

Poeschke, Joachim: Die Skulptur der Renaissance in Italien. Donatello und seine Zeit, Band 1, München 1990.

Schöne, Wolfgang: Zur Bedeutung der Schrägansicht für die Deckenmalerei des Barock, in: Festschrift für Kurt Badt, Berlin 1961, S. 144ff.

Winter, Gundolf: Durchblick oder Vision. Zur Genese des modernen Bildbegriffs am Beispiel von Robert Delaunays „Fenster Bildern“, in: Pantheon, Internationale Zeitschrift für Kunst, Heft 1 1984, S. 34ff. 


\section{Jens Schröter}

\section{Die Ästhetik der virtuellen Welt: Überlegungen mit Niklas Luhmann und Jeffrey Shaw}

\section{Das Phantasma}

Ab 1987 läuft im amerikanischen Fernsehen die Science Fiction-Serie Star Trek: The Next Generation. Eine der originellsten Einfälle ihrer Macher ist das so genannte ,Holodeck'. Das Holodeck ist ein Raum an Bord der Raumschiffe (und Raumstationen), in welchem eine begehbare, audiovisuell absolut ,realistische“, berührbare und olfaktorisch sowie gustatorisch erfahrbare Computersimulation erschaffen werden kann. Die ,Bilder' der Objekte sind einfach weitere Vorkommnisse der Objekte selbst. Diese Simulationen dienen als Entertainment, aber auch für Sport oder Kampftraining. In verschiedenen Episoden wird dargestellt, welche Gefahr vom Holodeck ausgehen kann, wenn die Sicherheitsvorrichtungen ausfallen und sich die Tür des Holodecks nicht mehr öffnen lässt: Schon in der dreizehnten Episode The Big Goodbye (USA 1987) kommt dabei jemand zu Tode. Anfang der 1990er Jahre war das Holodeck nicht nur eine populäre Fernsehfiktion, sondern mehr noch ein - wie bestimmte Formen der Techniksoziologie formulieren würden ${ }^{1}$ - ,Leitbild' der Forschung an virtuellen Räumen. Ein technisches und designerisches Handbuch über VR von 1993 benennt ganz selbstverständlich das Holodeck als ,ultimate goal‘ der VR-Forschung. ${ }^{2}$

Der Soziologe Niklas Luhmann schrieb in seinem Buch Die Kunst der Gesellschaft von 1995: „Der Raum macht es möglich, daß Objekte ihre Stellen verlassen. [...] Der Raum hat sein Prinzip darin, daß eine Stelle durch nur ein Objekt besetzt sein kann. “3 Durch die Materialität der simulierten Objekte ist auch diese letzte Bedingung im Holodeck gegeben. Das Holodeck bietet mindestens in dem Sinne eine virtuelle Welt, dass man sich erstens wirklich in ihr befindet und dass sie zweitens die materielle Widerständigkeit dessen bietet, was ,wir ${ }^{\star}$ die ,Welt ${ }^{\star}$ nennen. Doch in Wirklichkeit gibt es kein Holodeck und wird vielleicht niemals eines geben. Die ,virtuellen Welten“ mit denen wir es heute und in absehbarer Zukunft zu tun haben, sind anders.

1 Vgl. Dierkes/Hoffmann/Marz: Leitbild und Technik.

2 Vgl. Hamit: Virtual Reality and the Exploration of Cyberspace, S. 48/49.

3 Luhmann: Die Kunst der Gesellschaft, S. 181 und 182. Die von Luhmann ebenfalls diskutierte Zeit sei hier ausgeklammert. 


\section{Der virtuelle Raum, der reale Raum und die Ästhetik}

Was bedeutet virtuell? Es gibt eine Reihe von Vorschlägen, um diese Frage zu beantworten. Ich schlage hier eine an die Geschichte des Computers angelehnte Definition vor. Der Begriff ,virtuell' wird im Diskurs der Informatik zuerst im Kontext der Forschung an virtuellen Speichern (virtual memory) in den frühen 1960er Jahren verwendet. Virtuelle Speicher operieren mit der Trennung des logischen Adressraums vom tatsächlichen materiellen Speicherraum. Diese Dissoziation von logischer Struktur und materieller ,Basis‘ ist der Kern des Virtuellen. Die wissenschaftlich oder militärisch genutzte Computersimulation eines realen Objekts oder Prozesses besteht darin, dass mathematisch formalisierbare Strukturen von der Materialität des Objekts durch Vermessungen und Formalisierungen ,abgelöst ${ }^{6}$ werden, um dann als Grundlage eines virtuellen, approximativen und modifizierbaren Modells zu dienen. ${ }^{4}$ Das Modell prozessiert in Rechnern und wird auf verschiedenen Displays dargestellt. ${ }^{5}$

Ein virtueller Raum wäre also die von Materialität abgelöste, formalisierte, approximative, modifizierbare und in einem Rechner operative Struktur von Stellen, auf der sich Objekte bewegen können und die auf verschiedenen Displays dargestellt wird. Ein solcher Raum ist also buchstäblich Medium der „Messung und Errechnung von Objekten“6, wie Luhmann in etwas anderem Sinne bemerkt. Alle bisherigen Raumstrukturierungsverfahren ${ }^{7}$ können im Prinzip formalisiert und als virtuelle Struktur von Stellen simuliert werden. Genau das passiert, wenn man z.B. aus einem Grundriss der Villa Borghese in Rom ein virtuelles Modell eben dieser Villa erzeugt. Doch insofern das virtuelle Modell eben die Materialität des Modellierten per definitionem hinter sich lässt, ändert sich gerade die Räumlichkeit des Raums. In der so genannten wirklichen Welt bieten Wände qua ihrer Materialität - die auch im Luhmann im Kopf hat, wenn er schreibt: „Nur die Objekte selbst erschweren Bewegung ${ }^{\star 8}$ - Widerstand. Nicht so in einem virtuellem Modell z.B. der Villa Borghese. Nichts hindert im Prinzip den Blickpunkt daran, auch durch Wände, Boden und Objekte zu gleiten. Virtuelle Welten sind mithin erstens keine Welten, weil der Widerstand

4 Die Ablösung von der Materialität bezieht sich natürlich nur auf das virtuelle Objekt im Verhältnis zum Realobjekt, nicht aber auf die Hardware, die jedem Rechenprozess zugrundeliegt und z. B. die Rechnergeschwindigkeit determiniert. Vgl. Schröter: Computer/Simulation und Gramelsberger: Im Zeichen der Wissenschaften.

5 Vgl. zum Begriff des Displays bei Computern Thielmann/Schröter: Display II. Digital.

6 Luhmann: Die Kunst der Gesellschaft, S. 179.

7 Vgl. Luhmann: Die Kunst der Gesellschaft, S. 182 zum Verfahren der Grenzziehung.

8 Luhmann: Die Kunst der Gesellschaft, S. 182. 
der Objekte, also eine Segmentierung der Stellen, erst etabliert werden muss, statt immer schon vorausgesetzt werden zu können.

Zweitens aber sind virtuelle Welten daher keine Welten im konventionellen Sinne, da sie - anders als das Holodeck - in der Regel auf einem 2D oder mithilfe der Stereoskopie auf Pseudo-3D erweiterten Display erscheinen. Der Raum der so genannten virtuellen Welten ist nicht wie der Raum der Welt, der uns umgibt, sondern etwas, das vor uns erscheint. Natürlich wäre es auch anders denkbar, Virtual Reality war genau die Idee die Betrachter zu umgeben, aber der Konflikt zwischen der propriozeptiven Selbstwahrnehmung und der visuellen Wahrnehmung führte oft zu Schwindel und Unwohlsein - Simulatorkrankheit. Daher bleibt der Raum der virtuellen Welt in der Praxis letztlich eine Art Bildraum - und ist zumeist nach denselben zentralperspektivischen Regeln organisiert, wie schon der Bildraum der Malerei seit der Renaissance oder der Bildraum fotografischer Medien.

Bei so genannten virtuellen Welten muss man immer den Bildraum auf dem Display und den realen Raum der Betrachter zusammen untersuchen. Bei den im IAIS Fraunhofer verwendeten Displays wie der L-SHAPE etwa ${ }^{10}$ ist die systematische Verbindung von virtuellem Bildraum und Realraum offensichtlich. Vor dem Display gibt es eine Zone, die selbst als Projektionsfläche verwendet werden kann und die man daher nur mit Schlappen betreten darf. Diese Zone, in der die Betrachter sind, kann mithin je nach Modus selbst Teil des Bildraums sein. In diesem Falle kommt es zur Interferenz zwischen dem materialen Stellensystem und dem virtuellen Stellensystem. Während keine zwei Schlappen am selben Ort sein können, können sich sehr wohl eine Schlappe und projiziertes virtuelles Objekt am selben Ort befinden. Dieses Zusammentreffen muss nicht dem Zufall überlassen bleiben, sondern man könnte die Frage nach einer Gestaltung stellen, die solche Interferenzen des virtuellen und realen Stellensystems und der in ihnen lokalisierten Objekte gezielt nutzt. Diese Gestaltung müsste noch etwas beachten: Unabhängig davon, ob der Boden selbst zugleich Bild ist, bildet der Raum vor dem Display eine Arena der Betrachterbewegung. Die Betrachter können, sofern das Bild interaktiv veränderbar ist, sich wie in einem Tanz hin und her, vor und zurückbewegen und das Bild auszuloten. In der Praxis stehen die Betrachter jedoch oft ratlos und verwirrt herum. Dies zeigt: Die reale Betrachterbewegung ist Teil der virtuellen Welt. Eine Gestaltung der virtuell-realen Interferenz muss beachten, dass sie die Betrachterbewegung nicht unbotmäßig einschränkt, sondern vielmehr erst angemessen stimuliert. Wenn sie Betrachterbewegung erzielt, kann sie diese lenken und strukturieren (siehe 4.). Die Segmentierungen im virtuellen Raum und die im realen Raum können also in ein spezifisches Verhältnis gesetzt

9 Vgl. Husserl: Ding und Raum.

10 Vgl. Fraunhofer IAIS: TwoView/L-Shape. 
werden. Wenn ein solches Verhältnis dem Zweck dient, die Wahrnehmung der virtuellen Welt auf sich selbst zu lenken - also als Wahrnehmung der Wahrnehmung der Beziehung von realem und virtuellem Raum - dann ist die entsprechende Gestaltung ästhetisch. Zur Computersimulation bemerkte Martin Seel einmal: „Eine ästhetische Komponente kommt dabei erst ins Spiel, wenn es in der Ausführung und Wahrnehmung einer simulativen Darstellung nicht vorwiegend um das Simulierte, sondern zusätzlich oder auch vorwiegend um die Art der Simulation geht. “11

\section{Jeffrey Shaw: The Golden Calf (1994)}

Im Folgenden möchte ich exemplarisch eine solche ästhetische Gestaltung untersuchen, Jeffrey Shaws interaktive Installation The Golden Calf / Das goldene Kalb von 1994. Der 1944 in Melbourne, Australien geborene Shaw ist einer der wichtigsten Medienkünstler. Die Installation The Golden Calf besteht aus einer konischen, im Querschnitt viereckigen und ca. $1 \mathrm{~m}$ hohen Säule, auf der ein Farbdisplay liegt, welches mit der Säule durch ein dickes schwarzes Kabel verbunden ist. Das Farbdisplay zeigt einen abstrakten, leeren Raum, in der sich eine vergleichbare virtuelle Säule befindet. Auf der virtuellen Säule steht ein virtuelles goldenes Kalb. ${ }^{12}$ Das titelgebende goldene Kalb ist natürlich eine Anspielung auf die berühmte Bibelstelle Exodus 32, 1-4:

1 Als das Volk sah, dass Mose noch immer nicht vom Berg herabkam, versammelte es sich um Aaron und sagte zu ihm: Komm, mach uns Götter, die vor uns herziehen. Denn dieser Mose, der Mann, der uns aus Ägypten heraufgebracht hat - wir wissen nicht, was mit ihm geschehen ist.

$[\cdots]$

3 Da nahm das ganze Volk die goldenen Ohrringe ab und brachte sie zu Aaron.

4 Er nahm sie von ihnen entgegen, zeichnete mit einem Griffel eine Skizze und goss danach ein Kalb. Da sagten sie: Das sind deine Götter, Israel, die dich aus Ägypten heraufgeführt haben.

[...]

19 Als Mose dem Lager näher kam und das Kalb und den Tanz sah, entbrannte sein Zorn. Er schleuderte die Tafeln fort und zerschmetterte sie am Fuß des Berges. ${ }^{13}$

11 Seel: Ästhetik und Aisthetik, S. 25/26.

12 Das Kalb ist allerdings eher eine ausgewachsene Kuh.

13 Siehe Bibelwerk: Die Bibel. 


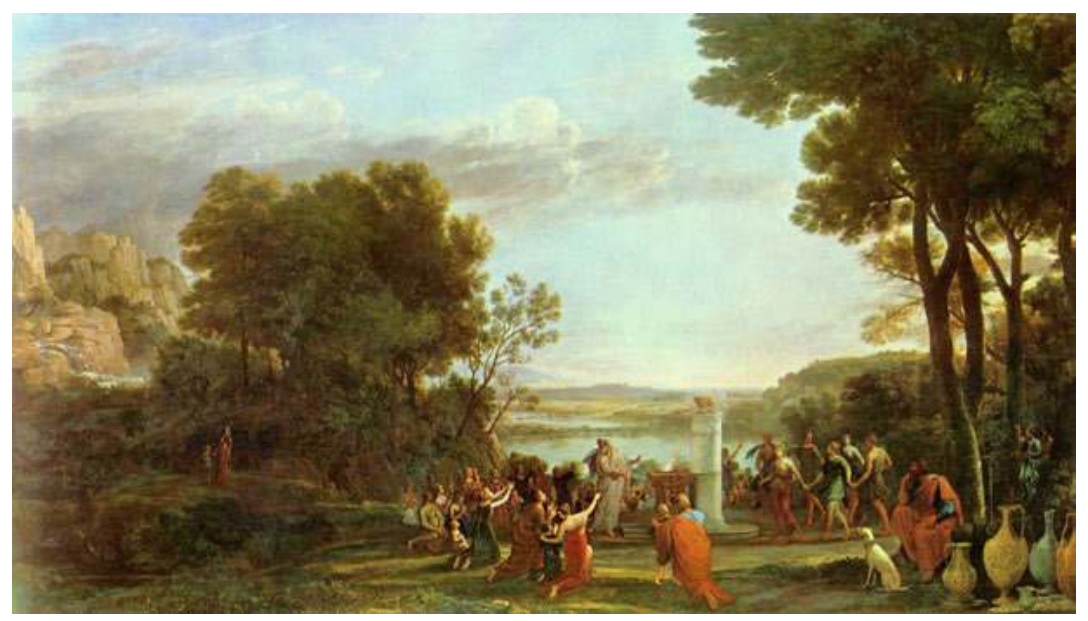

Abbildung 1: Claude Lorrain, Landschaft mit der Anbetung des goldenen Kalbes

Diese Bibelstelle wurde in verschiedenen Werken der Kunst verarbeitet, so z.B. in Claude Lorrains Landschaft mit der Anbetung des goldenen Kalbes. ${ }^{14}$

Doch während die Betrachter von Lorrains Bild weitgehend unbewegt das Bild betrachten dürften - von gelegentlichen Annäherungen, um die Textur des Gemäldes in Augenschein zu nehmen, abgesehen -, bewegen sich die Betrachter von Shaws Installation intensiv. Gleich den Israeliten tanzen sie um das Kalb. Doch anders als bei Lorrain befindet sich das Kalb gar nicht in demselben Raum, wie jene, die um es tanzen. Es ist in einem anderen Raum, der gleichwohl den realen Raum der Betrachter überlagert.

Die Betrachter (oder Benutzer?) haben ein zentralperspektivisch organisiertes fenestra aperta (Alberti) in der Hand. Anders als in der Malerei hängt es nicht distanziert an der Wand. Die Betrachter bewegen das Fenster und versuchen so das System der virtuellen Stellen, das sich außerhalb des Displays unsichtbar über den realen Raum erstreckt, auszuloten. Man kann versuchen, den virtuellen mit dem realen Raum zur Deckung zu bringen. Man kann versuchen, den Blick auf die wirkliche Säule mit dem auf die virtuelle Säule zu korrelieren, doch das wird nicht gelingen, schon weil die virtuelle Säule weniger konisch als die reale Säule ist. Die Räume sind getrennt - und doch verbunden, wie das dicke schwarze Kabel deutlich macht. Es begrenzt mit seiner Länge die mögliche Entfernung der Betrachter, wodurch die reale Säule den Punkt im Realraum markiert, auf den die gesamte Interferenz bezogen bleibt. Das Display, das Kabel und die reale Säule sind Objekte, deren Stellen nicht von anderen Objekten besetzt werden können, während das virtuelle Kalb virtuell genau dort steht, wo im Ausgangs- und

14 1653, Öl auf Leinwand, $142 \times 248$ cm, Kunsthalle Karlsruhe. 

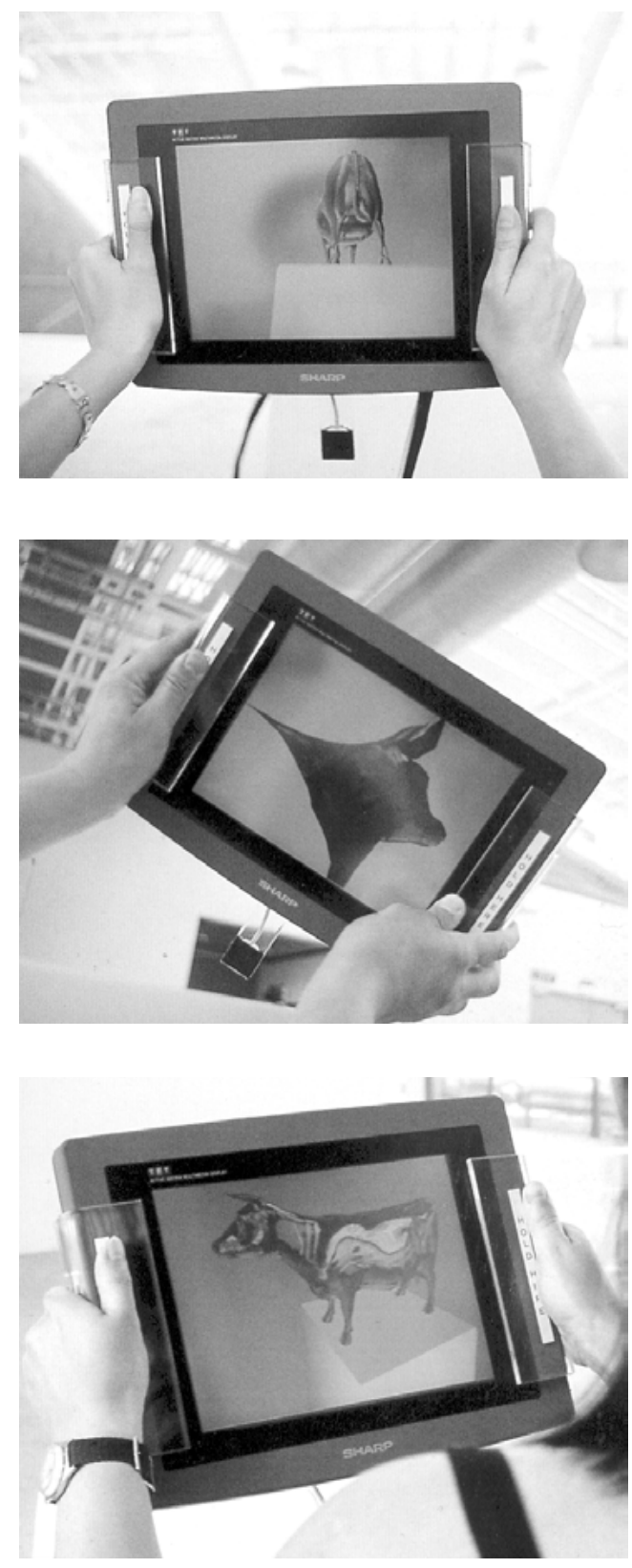

Abbildung 2: Nutzungssequenz von The Golden Calf ${ }^{15}$ 
Endzustand der Installation realiter das Display ruht. Das Display und das Kalb nehmen an diesen Extrempunkten, vor und nach dem Tanz der Betrachtung, denselben Raum ein. Die Reflexion auf die Beziehung zwischen dem realen Umraum und dem virtuellen Raum wird noch dadurch gesteigert, dass auf dem virtuellen goldenen Kalb Reflexionen des jeweiligen Aufstellungsortes der Installation zu sehen sind. Shaw macht digitale Fotos des Umraums, die je nach Blickwinkel der Betrachter auf das virtuelle Modell gemappt werden. Das Kunstwerk liefert eine „Imitation der Differenzstruktur von Raum“, wie man mit Luhmann sagen könnte. ${ }^{16}$ Die Betrachter werden genötigt, den virtuellen Raum und seine Relation zum realen Raum und ihre Bewegungen darin selbst zu beobachten - ein „selbstbezügliche[s] Erscheinen“, wie Seel es nennt. ${ }^{17}$

Das goldene Kalb selbst ist Teil der Demosoftware der Silicon Graphics Workstation, die der Installation zugrunde liegt - und verweist insofern selbstreflexiv auf die technisch-institutionelle Quelle der Computergraphik. Zugleich ist die historische Vor-Schrift, aus der das Motiv stammt - Exodus 32 - ja selbst eine Reflexion auf den Kampf zwischen den Medien Bild und Schrift. Das Judentum als Schriftreligion - nicht umsonst heißt noch die Bibel Heilige Schrift - kämpft in Form der von Gott selbst geschriebenen Tafeln gegen den Götzen, der ein Bild ist. Aaron ,zeichnete mit einem Griffel eine Skizze und goss danach ein Kalb'. So gesehen ist der Tanz, den die Betrachter um das virtuelle goldene Kalb aufführen, ein Tanz um den Götzen des virtuellen Bildes. 1994 als Shaw diese Installation erarbeitete, waren die aufs Maßloseste übersteigerten Utopien um die Virtuelle Realität, die sich besonders am Anfang der 1990er Jahre nicht nur im Holodeck, sondern auch in vielen anderen Verlautbarungen zeigten, noch nicht ganz verklungen. ${ }^{18}$ Shaw zeigt in der Verbindung der Reflektion auf die Differenzstruktur zwischen realem und virtuellem Raum mit der Kritik an den Utopien um den virtuellen Raum, dass letzterer eben keine ganz andere virtuelle ,Welt' ist, sondern Teil der realen Welt, in der es von der je und je verschiedenen Gestaltung abhängt, was wie den Betrachtern begegnet. Denn schließlich ist das virtuelle goldene Kalb ein Bild, das auf der operativen Schrift des Pro-Gramms, was ja nichts anderes als Vor-Schrift bedeutet, beruht. Diese Vor-Schrift verlängert sich in jene auf dem Display selbst, die den Betrachtern erklärt, was sie zu tun haben, um die Installation überhaupt benutzen zu können.

15 Aus Dinkla et al.: InterAct!

16 Luhmann: Die Kunst der Gesellschaft, S. 184. Vgl. Qvortrup: Cyberspace as Representation of Space Experience

17 Seel: Vor dem Schein kommt das Erscheinen, S. 781.

18 Vgl. Schröter: Das Netz und die Virtuelle Realität, S. 152-276. 

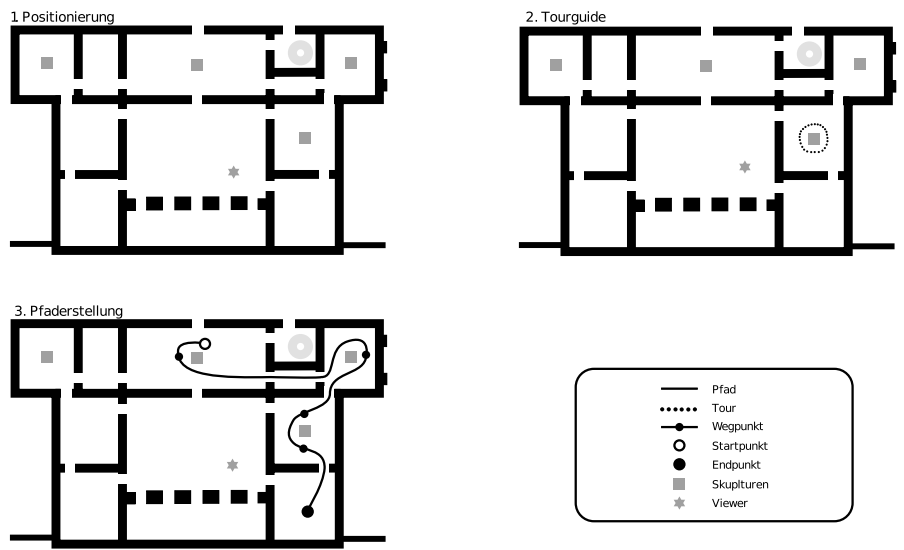

Abbildung 3: Abbildung eines Steuerungsmenus für den virtuellen Raum am Beispiel der virtualisierten Villa Borghese ${ }^{19}$

\section{Der virtuelle Betrachter und die Atmosphäre}

Shaws Installation verweist aber nicht nur auf die Differenz und Interferenz von realem und virtuellem Raum, sondern auch auf jene von virtuellem und realem Betrachter. Zunächst leuchtet das kaum ein. Denn bei Shaw, wie z.B. auch bei der freien Interaktion mit der L-SHAPE, wechselt der Blickpunkt auf die virtuellen Objekte mit der Bewegung der Betrachter. Realer Blick und virtueller Blickpunkt werden eins. Doch: Ich hatte darauf hingewiesen, dass Shaw digitalisierte Fotos des Umraums nutzt, um diesen im Kalb zu spiegeln. Die Betrachter werden dabei natürlich nicht mitgespiegelt, denn die Fotos entstehen vor der realen Betrachtungssituation. Zugleich aber spiegeln sich die Betrachter und je nach Lichtverhältnissen der reale Raum, in dem sie sich bewegen, in der glänzenden Scheibe des Displays. Shaw zeigt uns subtil eine Differenz zwischen realem und virtuellem Blick auf. Diese Differenz erlaubt wieder eine Reihe von Gestaltungen.

So kann der virtuelle Raum auch durch eher filmische Formen gestaltet werden. Die Interaktion kann auf ein Interface ausgelagert werden, auf dem Wege beschrieben werden, die eine kameraähnliche Fahrt durch den virtuellen Raum auslösen (siehe Abb. 3). Die Bewegung des virtuellen Blickpunkts wird von der realen Bewegung dissoziiert. ${ }^{20}$ Spätestens jetzt entfällt die Betrachterbewegung (weitgehend). Der Blick wird an einen virtuellen Betrachter delegiert, der zugleich

19 Jansen: Kollaborative Interaktion in virtuellen Umgebungen

20 Vgl. zu dieser Verwendung des Dissoziationsbegriffs Dobbe: Dispositive des Sehens. 
ein idealer Betrachter sein kann (aber nicht muß) - in dem Sinne, dass der ideale Betrachter Weisen der Betrachtung exemplifiziert. Die Einrichtung eines solchen idealen Betrachters kann zumal in kunsthistorischen Zusammenhängen als notwendig erachtet werden, insofern ein zentrales epistemologisches Verfahren der Disziplin Kunstgeschichte in der Produktion idealer Betrachter besteht, wie z.B. in meiner Analyse von Shaws Installation. Ich stützte mich dabei ja nicht auf den - etwa empirisch vermessbaren - Durchschnitt dessen, was faktische Museumsbesucher erfahren, sondern auf das, was man idealiter erfahren könnte. ${ }^{21}$ Insofern also in einem virtuellen Raum selbst Objekte dargestellt werden, die als ästhetische eine im Sinne Seels selbstbezügliche Wahrnehmung ermöglichen, kann es notwendig sein die Betrachtungsbedingungen, die von dem Objekt vorausgesetzt werden, mit darzustellen. Luhmann schreibt: „Auch eine Skulptur definiert den Raum um sie herum“ und näherhin: „Der imaginäre Raum wird nach außen projiziert in der Form von Einteilungen, die das Kunstwerk ihm vorschlägt. “22 Diese Veränderung des Raums durch Objekte auch an den Stellen, wo das Objekt nicht ist, nennt Luhmann die Atmosphäre: „Ein besetzter Raum lässt Atmosphäre entstehen. Bezogen auf die Einzeldinge, die Raumstellen besetzen, ist Atmosphäre jeweils das, was sie nicht sind, nämlich die andere Seite ihrer Form; also auch das, was mitverschwinden würde, wenn sie verschwänden.“ Und weiter: Atmosphäre „entsteht dadurch, daß jede Stellenbesetzung eine Umgebung schafft, die nicht das jeweils festgelegte Ding ist, aber auch nicht ohne es Umgebung sein könnte. “23 Die Verschiebung der Stellenrelationen durch Besetzung mit Objekten oder durch das Einrichten von Segmentierungen ist nicht unabhängig vom Betrachter. Er (oder Sie) ist der Bezugspunkt, von dem aus die Relationen sichtbar werden. Wird ein architektonisch-skulpturales Ensemble wie Berninis Gruppen in der Villa Borghese virtuell rekonstruiert, muss nicht nur die Umgebung, sondern auch eine Bahn (oder mehrere Bahnen) der Betrachter ideal rekonstruiert werden. Andernfalls verschieben sich die Stellen-Relationen - und mithin auch die Atmosphäre. Virtuelle Umgebung und virtueller Betrachter - die beiden Elemente der virtuellen Atmosphäre bedingen sich wechselseitig. Je mehr aber die Atmosphäre des virtuellen Raums stabilisiert wird, desto mehr trennt sich der virtuelle vom realen Raum und vom Tanz des Betrachters. Doch selbstredend können auch die Objekte im virtuellen Raum auf die Stellen des realen Raums und die Objekte in diesem bezogen werden. Auch diese Interferenz bildet eine Atmosphäre. In diesem Fall kann wiederum die Führung des Betrachters vom idealen-virtuellen Betrachter

21 Natürlich ist meine Beschreibung auch nur eine Annäherung an die potentielle Fülle des Erfahrbaren.

22 Luhmann: Die Kunst der Gesellschaft, S. 183 und 79. Vgl. in diesem Sinne auch schon Heidegger: Die Kunst und der Raum

23 Luhmann: Die Kunst der Gesellschaft, S. 181. 
mindestens teilweise auf die Führung des Betrachters durch Objekte im realen Raum verlagert werden.

\section{$5 \quad$ Fazit}

Die virtuelle Welt ist ein komplexes Ge-Bilde. Mit ,komplex‘ meine ich damit etwas Ähnliches, wie wenn man in der Mathematik von ,komplexen Zahlen`als Verbindung von reellen Zahlen und der imaginären Einheit i spricht. ${ }^{24}$ Denn die komplexe Raumbildlichkeit der virtuellen Welt besteht aus den Interferenzen von

1. realem und virtuellem Raum,

2. realen und virtuellen Objekten, sowie von

3. realem und virtuellem Betrachter.

Diese drei mal zwei Parameter können moduliert und zu Atmosphären verbunden werden. ${ }^{25}$ Nichts gibt im Prinzip vor, welche Modulationen vorzunehmen und welche Atmosphären herzustellen sind. Es ist zu vermuten, dass es bestimmte Modulationen und Atmosphären gibt, die den Betrachtern eine einfache und funktionale Erfahrung ermöglichen - wie in dem unmöglichen Grenzfall des perfekten Holodecks, in dem real und virtuell auf allen Ebenen einfach zusammenfallen. Andere Modulationen und Atmosphären komplizieren und vervielfältigen hingegen die Erfahrungen und führen zu einem ästhetischen „Abenteuer im Beobachten“26 (wie in der Installation Shaws). Das Leitbild des ,Holodecks' engt diese Vielfalt von Erfahrungen eher ein - wozu sollte eine virtuelle Welt denn genauso wie die ,reale Welt' sein, selbst wenn dies möglich wäre? Schon Virginia Woolf soll bemerkt haben: „Kunst ist keine Kopie der wirklichen Welt. Ein solch verdammtes Ding ist genug. “27 In der Tat: Statt die wirkliche Welt in einer, virtuellen Welt' $\mathrm{zu}$ wiederholen, ist es doch viel interessanter die vielfältigen Spielräume der Gestaltung auszuloten. Dies bleibt eine unerschöpfliche Aufgabe - für Informatiker wie für Künstler.

24 Vgl. Wikipedia: Komplexe Zahl.

25 Zusätzlich könnten auch akustische Parameter eine Rolle spielen, vgl. grundsätzlich Volmar: Die Anrufung des Wissens.

26 Luhmann: Die Kunst der Gesellschaft, S. 227.

27 Zit. in: Goodman: Sprachen der Kunst, S. 15. 


\section{Literatur}

Bibelwerk: Die Bibel, 〈URL: http://alt.bibelwerk.de/bibel/〉 - Zugriff am 17.01.2008.

Dierkes, Meinolf/Hoffmann, Ute/Marz, Lutz: Leitbild und Technik. Zur Entstehung und Steuerung technischer Innovationen, Berlin 1992.

Dinkla, Söke et al. (Hrsg.): InterAct! Schlüsselwerke interaktiver Kunst, Ausstellung Wilhelm Lehmbruck Museum Duisburg, Ostfildern 1997.

Dobbe, Martina: Dispositive des Sehens. Anmerkung zur Skulptur, in: Winter, Gundolf et al. (Hrsg.): Skulptur - zwischen Realität und Virtualität, München 2006, S. 103-124.

Fraunhofer IAIS: TwoView/L-Shape, 〈URL: http://www.iais.fraunhofer.de/ 645.html?\&L=0 - Zugriff am 16.12.2008.

Goodman, Nelson: Sprachen der Kunst. Entwurf einer Symboltheorie, Frankfurt a.M. 1995.

Gramelsberger, Gabriele: Im Zeichen der Wissenschaften. Simulation als semiotische Rekonstruktion wissenschaftlicher Objekte, in: Grube, Gernot et al. (Hrsg.): Schrift. Kulturtechnik zwischen Auge, Hand und Maschine, München 2005, S. 439-452.

Hamit, Francis: Virtual Reality and the Exploration of Cyberspace, Carmel 1993.

Heidegger, Martin: Die Kunst und der Raum, St. Gallen 1969.

Husserl, Edmund: Ding und Raum. Vorlesungen 1907, Hamburg 1991.

Jansen, Tim: Kollaborative Interaktion in virtuellen Umgebungen im kunsthistorischen Kontext, Diplomarbeit, FH-Nürnberg 2007.

Luhmann, Niklas: Die Kunst der Gesellschaft, Frankfurt a.M. 1995.

Qvortrup, Lars: Cyberspace as Representation of Space Experience: In Defence of a Phenomenological Approach, in: ders. (Hrsg.): Virtual Space. Spatiality in Virtual Inhabited 3D Worlds, London u.a. 2002, S. 5-24.

Schröter, Jens: Computer/Simulation. Kopie ohne Original oder das Original kontrollierende Kopie? in: Fehrmann, Gisela et al. (Hrsg.): OriginalKopie Praktiken des Sekundären, Köln 2004, S. 139-155.

Schröter, Jens: Das Netz und die Virtuelle Realität. Zur Selbstprogrammierung der gesellschaft durch die universelle Maschine, Bielefeld 2004. 
Seel, Martin: Vor dem Schein kommt das Erscheinen. Bemerkungen zu einer Ästhetik der Medien, in: Merkur, 47 1993, Nr. 9/10 (=Nr. 534/535), S. 771-783.

Seel, Martin: Ästhetik und Aisthetik. Über einige Besonderheiten ästhetischer Wahrnehmung, in: Recki, Birgit/Wiesing, Lambert (Hrsg.): Bild und Reflexion. Paradigmen und Perspektiven gegenwärtiger Ästhetik, München 1997, S. $17-38$.

Thielmann, Tristan/Schröter, Jens (Hrsg.): Display II. Digital, Marburg 2007.

Volmar, Axel: Die Anrufung des Wissens. Eine Medienepistemologie auditorischer Displays und auditiver Wissensproduktion, in: Thielmann, Tristan/ Schröter, Jens (Hrsg.): Display II. Digital, Marburg 2007, S. 105-116.

Wikipedia: Komplexe Zahl, 〈URL: http://de.wikipedia.org/wiki/Komplexe_ Zahl $\rangle$ - Zugriff am 17.01.2008. 


\section{Steffi Beckhaus}

\section{Zwischen real und digital: Intuitive, reichhaltige und freudvolle Schnittstellen}

\section{Der Mensch, die Schnittstelle und die virtuelle Welt}

Ein Mensch kann mit dem Computer nur über dessen Ein- und Ausgabeschnittstellen in Kontakt treten. Virtuelle Welten, unabhängig ob es sich um 3D Repräsentationen der realen Welt im Museums- und Kulturkontext oder um Spiele, Internet oder Office Anwendungen handelt, werden hauptsächlich visuell abgebildet und Interaktionen mit ihnen über Handeingabegeräte ermöglicht. Dies gilt auch für die meisten Systemkonfigurationen: Desktop Systeme (PCs) unterscheiden sich nicht wesentlich in ihren Ein- und Ausgabemodalitäten von einem Großteil der Systeme, die immersive Virtuelle Realitäten (VR) projizieren. Standard ist, dass die meisten virtuellen Welten sowohl bei PCs als auch in der VR nur über das Auge wahrgenommen und über die Finger bzw. die Hand beeinflusst werden.

Der Mensch als Rezipient und Akteur in der realen wie in der virtuellen Welt ist jedoch ein multisensorisches Wesen mit vielfältigen Fähigkeiten zur Rezeption und Aktion.

Unter diesem Blickwinkel beschäftigt sich die aktuelle Forschung am im.ve Labor des Departments Informatik der Universität Hamburg damit, Basistechnologien für virtuelle Welten zu erforschen und prototypisch Schnittstellen zu entwickeln, welche neben Funktionalität auch ein umfassendes, freudvolles und reichhaltiges Erlebnis des Menschen ermöglichen.

Dieser Beitrag motiviert zunächst unseren Ansatz reichhaltige Schnittstellen zu entwickeln. Er diskutiert menschliche Wahrnehmung der realen Welt im Unterschied zum Erleben virtueller Welten und die Rolle, die eine Schnittstelle in der Vermittlung zwischen beiden Welten spielt. Anschließend zeigen zwei Projekte beispielhaft, was mit intuitiv, freudvoll und reichhaltig gemeint ist. Beide wurden im Rahmen unserer Forschung zu interactive experiences entwickelt. Das erste Beispiel behandelt das ChairIO, eine sitzbasierte Schnittstelle zur Steuerung der Bewegung durch virtuelle Welten. Das zweite Beispiel zeigt anhand der GranulatSynthese Installation Möglichkeiten multisensorischer Steuerung interaktiver Tische. Beide Ansätze verbinden auditive, haptische und kinästhetische Elemente im realen Raum mit elektronischen Daten und virtuellen Welten im Computer. 


\subsection{Unsere Wahrnehmung der realen, materiellen Welt}

Die Welt um uns herum liefert uns reichhaltige sensorische Eindrücke. Wir sehen, hören, fühlen, riechen und schmecken - und das gleichzeitig und jederzeit.

Unsere bewusste Aufmerksamkeit ist dabei oft auf nur einen Ausschnitt dieses Angebots gerichtet. Wenn wir beispielsweise ein Buch lesen, dann fokussieren wir hauptsächlich auf die geschriebenen Worte, auf Sätze und deren Bedeutung. Visuell erfassen wir die Buchstaben und mental tauchen wir in die Erzählung ein. Dennoch beeinflusst das materielle Buch selbst unser Erleben: wir fühlen das Taschenbuch, den Folianten, sein Gewicht. Wir riechen die Blätter des alten oder neuen Buches und hören das Rascheln der Seiten beim Umblättern. Zur gleichen Zeit nehmen wir wahr, wie und worauf wir sitzen oder stehen, ob die Luft um uns herum warm oder kühl ist und in welcher Umgebung wir uns befinden. Zusammen mit vielen anderen sensorischen, kontextbezogenen und emotionalen Komponenten bestimmen diese Eindrücke potentiell unser Erleben - selbst wenn diese Informationen teilweise unwichtig sind oder unterbewusst wahrgenommen werden.

Worauf wir unsere Aufmerksamkeit lenken wollen, entscheiden wir persönlich in jedem Moment erneut und auf Basis des zur Verfügung stehenden Angebots und des aktuellen Interesses: auf die Geschichte im Buch, auf das Layout, auf Bilder oder auf Personen, die gerade den Raum betreten haben. Diese Entscheidungsfreiheit versucht ein Autor zu lenken, indem er Inhalt oder Präsentation optimiert; verhindern kann er sie jedoch nicht. Als aktive Teilnehmer in der realen wie der virtuellen Welt entscheiden wir frei und unerzwingbar, was wir aus der gegebenen Vielfalt eines Angebotes zu jeder Zeit wabrnebmen wollen.

\subsection{Die programmierte virtuelle Welt}

Die virtuelle Welt ist, im Gegensatz zur realen Welt, ein programmiertes geistiges Produkt. Sie ist in ihrer Beschreibung nicht materiell, sieht man von dem binären Code auf dem Speichermedium ab. Sie bleibt solange eine Vision, eine Idee, bis sie abgebildet wird. Materiell erfahrbar ist sie selbst jedoch nicht.

Damit also virtuelle Dinge berührbar, greifbar, beweglich, modifizierbar werden, muss dies zunächst explizit programmiert werden. ${ }^{1}$ Jedes Objekt, jede Materialeigenschaft, jedes Verhalten unter Berücksichtigung der physikalischen Gesetze muss erfasst und beschrieben werden. Die Vielfältigkeit, die wir in der realen Welt gewohnt sind, muss in der virtuellen Welt zunächst datentechnisch hinterlegt werden, damit sie anschliessend für die aktuelle Situation aufbereitet und dem Menschen vermittelt werden kann.

1 Schröter beschreibt dieses ausführlich in seinem Kapitel (S. 25ff.) 


\subsection{Die Schnittstelle als (einziger) Kontakt zur virtuellen Welt}

Die virtuelle Beschreibung, die diskret oder funktional im Computer vorliegt, muss also erlebbar gemacht werden. Wie perfekt auch immer die virtuelle Welt ausgedacht und dann modelliert sein mag, ihre Abbildung kann nur so gut sein, wie die eingesetzten Systeme. Sie ist abhängig von der Art, Qualität und Beschaffenheit der vermittelnden Displays, der Interaktionsgeräte und der Interaktionstechniken. Die Vermittlung der virtuellen Welt und ihre Abbildung auf die real erfahrbare Welt übernehmen in der Regel die zuvor beschriebenen Schnittstellen. Dies sind beispielsweise in Desktop Systemen ein Monitor für die visuelle Ausgabe, Maus und Keyboard für die Steuerung der Anwendung. Selbst technologisch fortgeschrittene Varianten immersiver stereo-projizierter Displays wie $\mathrm{CAVE}^{\mathrm{TM}}$, i-Cone ${ }^{\mathrm{TM}}$ oder ähnliche den Benutzer umgebende Installationen fokussieren auf visuelle Displays. Die immersiven Systeme werden gesteuert von standardisierten Hand-Eingabegeräten wie einem Flying Joystick. Akustische oder haptische Displays existieren, werden aber nur von Fall zu Fall eingesetzt.

Technische Einschränkungen in der Abbildung, beispielsweise Einschränkungen in Qualität, Auflösung, Wiederholrate, Verzögerung, Blickfeld, dem Interaktionsraum oder den unterstützen Modalitäten, beeinträchtigen auch unser Erleben erheblich. Wenn nur visuelle Informationen verfügbar gemacht werden, kann das Erleben der virtuellen Welt auch nur über den visuellen Sinneskanal erfolgen. Der technische Anteil der Display- und Interaktionsgeräte ist allerdings voll-sensorisch erfahrbar durch deren Geräusche, Wärme und Vibrationsentwicklung. Um die Aufmerksamkeit voll auf die präsentierte virtuelle Welt richten zu können und die wichtigsten inhaltlichen Informationen durchzulassen, bedarf es eines bewussten Ausblendens dieser oft dominanten Störungen.

Insgesamt kann man sagen, dass ein Computer mit den aktuellen technischen Möglichkeiten bislang eine nur sehr eingeschränkte Abbildung der Welt erreicht. Mit dem momentanen Stand der Technik kann keine voll-sensorischer interaktive virtueller Welten abgebildet werden und die Umsetzung der Vision von Star'Trek's Holodeck ist weit entfernt. Der Fokus aktueller Forschung im Bereich VR lässt auch nicht auf eine schnelle Entwicklung hoffen. Grundsätzlich ist überhaupt fraglich, ob ein Holodeck das letztendliche Ziel der Entwicklung virtueller Welten sein muss. ${ }^{2}$

\subsection{Perspektiven und Möglichkeiten}

Anders, als die technische Betrachtungsweise der letzten beiden Abschnitte, sähe die Sichtweise aus der Perspektive eines Menschen aus, der, wie im Abschnitt 1.1 untersucht wurde, mit all seinen Sinnen und Fähigkeiten arbeiten und erleben

2 Vgl. mit dem Kapitel von Schröter (S. 27) 
möchte. Aktuelle Schnittstellen sind weit davon entfernt, dem Menschen das Angebot an Informationen zu vermitteln, das er von seinem sonstigen Erleben in der realen Welt gewohnt ist. Dieses eingeschränkte Angebot an Schnittstellen oder deren Erlebnisqualität stellen auch aktuelle Anwendungsentwicklungen meist nicht in Frage. Selbst Design Methoden, die den Menschen in den Mittelpunkt der Entwicklung stellen ${ }^{3}$, hinterfragen meist nicht die Standardkonfigurationen und -benutzung der aktuellen Systeme, die in der Regel den Menschen, die Schnittstelle und die virtuelle Welt sauber trennen und eine Erfahrung der virtuellen Welt oder ihrer Steuerung nur von der Ferne und als geistige Übung zulassen. Die virtuelle Welt muss im Kopf entstehen, da sie nicht wie die reale Welt über die Sinne erfahrbar ist. Sie wird dadurch auch nur geistige Qualitäten annehmen können und negiert die voll-sensorischen Fähigkeiten und möglichen Bedürfnisse des Menschen. Hier ist die materielle Welt im Vorteil: Alles Materielle um uns herum präsentiert sich uns unmittelbar, vollständig und reichhaltig, während alles Virtuelle erst programmiert und abgebildet werden muss und die Abbildung selber neue unerwünschte Reize hinzufügt.

Da Computerarbeit allgegenwärtig in Beruf und Freizeit unser Leben bestimmt und immer öfter auch das Lernen und Arbeiten in virtuelle Welten verlagert wird, lohnt sich das Nachdenken über Alternativen.

Eine Möglichkeit wäre das bewusste Einbeziehen der realen Welt in die Installation und Interaktion. Hierdurch wird das Erleben für den Menschen automatisch sensorischer, potentiell intuitiver und bedienbarer. Während die virtuelle Welt lediglich durch die Vermittlung einer Schnittstelle mit deren eingeschränkten programmierten Möglichkeiten erfahren wird, sind alle Objekte der realen Welt automatisch voll präsent, multimodal und multisensorisch. Zugrunde liegt die Idee, Technik nicht nur als einen Vermittler und als potentielles Hindernis zu sehen, sondern Technik und reale, wirkliche, materielle Gegenstände aktiv mit in das Erleben der virtuellen Welt zu integrieren. Mit Bewegungssensoren in aktuellen mobilen Geräten und weiteren fortschrittlichen Technologien haben wir heute großes Potential, dem Computer wieder jene Beschränkungen zu nehmen, die ihm die Reduktion auf Auge und Hand auferlegt hat ${ }^{4}$. Indem man aktiv mit allen Komponenten umgeht und diese zu einem Gesamterlebnis komponiert, hat man die Möglichkeit multisensorische und multimodale Informationen zu vermitteln, ohne diese selbst in der virtuellen Welt modellieren zu müssen.

3 Vgl. User Centered Design in Rubin: Handbook of Usability Testing.

4 Dies gilt gleichermaßen für PC wie VR Systeme, obwohl die technologischen Möglichkeiten theoretisch durch die Tracking Geräte in VR Systemen vorher schon bestanden haben, jedoch vermutlich auch aus Kostengründen nicht konsequent eingesetzt wurden. 

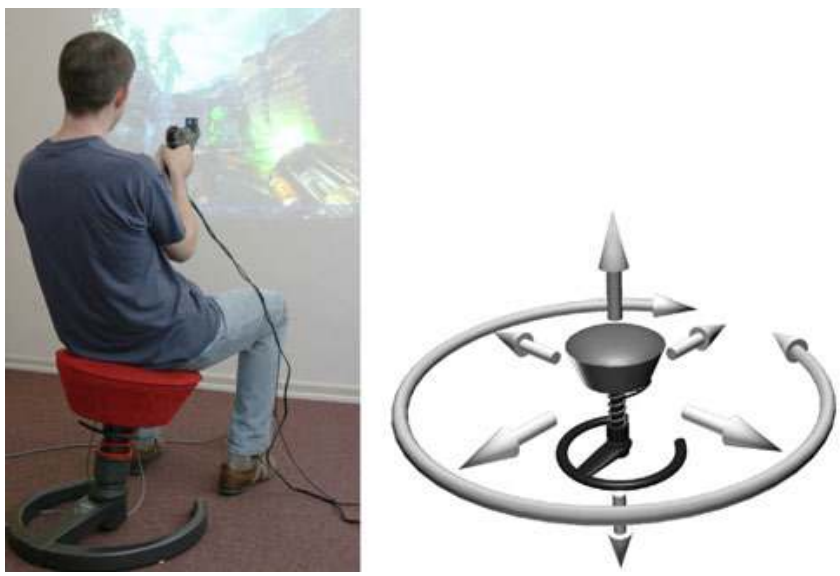

Abbildung 1: Das ChairIO als Spiele Device mit einem zusätzlichen Handeingabegerät. Rechts sind die Bewegungs- und damit Steuermöglichkeiten des ChairIO's dargestellt. Hier wird der Swopper ${ }^{\top \mathrm{M}}$ from aeris-Impulsmöbel $\mathrm{GmbH}$ als Basisstuhl des ChairIO eingesetzt.

Die folgenden zwei Beispiele demonstrieren erste Möglichkeiten in diese Richtung. Das erste befasst sich direkt mit der Steuerung virtueller Welten während das zweite neue mögliche Interaktionsparadigmen auf interaktiven Tischen aufzeigt.

\section{Das ChairlO - Steuerung in virtuellen Welten}

Ein Beispiel für eine Computerschnittstelle, die gleichermaßen für Desktop Systeme und große Projektionen eingesetzt werden kann, ist das ChairIO. Es steuert intuitiv die Bewegung durch virtuelle Welten und bietet gleichzeitig ein reichhaltiges Bewegungserlebnis für den Akteur selbst.

Das ChairIO ist eine sitzbasierte Computerschnittstelle, die aus einem mit Sensoren ausgestatteten beweglichen Bürohocker besteht. Die Sensoren nehmen die Bewegung des Hockers auf und übermitteln sie an den Computer. Der Computer übersetzt dann diese Informationen beispielsweise in Bewegung in einer 3D Welt oder in Steuerungsinformation für Spiele oder GoogleEarth. Abb. 1 zeigt das ChairIO im Einsatz als Steuerung eines Spiels. ${ }^{5}$

5 Das ChairIO als Steuerungsgerät eines First-Person-Shooter Spiels wird ausführlich beschrieben in Beckhaus/Blom/Haringer: A new gaming device for a First-PersonShooter und allgemeiner in Beckhaus/Blom/Haringer: ChairIO - the Chair-Based Interface. 


\subsection{Die Steuerung virtueller Welten mit einem Stuhl}

Als Stuhl verwenden wir den Swopper ${ }^{\mathrm{TM}}$ von der Firma Aeris. ${ }^{6}$ Dieser Bürostuhl hat eine bewegliche Mittelsäule, die federnd gelagert ist. Er funktioniert also vergleichbar einem stossdämpfer-gefedertem Joystick, auf dem man selbst sitzt. Man kann den Stuhl in beide horizontale Richtungen dynamisch auslenken und gleichzeitig auf ihm auf- und ab-federn. Die Sitzfläche rotiert frei, Feder und Gelenk des Stuhls können eingestellt und individuell für jede Person in Höhe und Dämpfung angepasst werden.

Ein solcher Stuhl ist ein idealer Kandidat, um Bewegung in virtuellen Welten zu vermitteln. Bewegung durch eine virtuelle Welt bedeutet für den Computer die Steuerung des Blickpunktes des Benutzers innerhalb der 3D Welt. Dazu braucht er zwei Fähigkeiten: einerseits das Umschauen - man steht an einem Punkt und dreht sich um die eigene Achse - und andererseits die Veränderung des Standortes - man bewegt sich auf der Bodenfläche vorwärts, rückwärts, seitwärts und gegebenenfalls auch auf und ab. Man benötigt als Benutzer also sowohl Kontrolle über die Rotation als auch über mehrere Richtungen.

Das ChairIO auf Basis des Swoppers unterstützt genau diese Freiheitsgrade durch die Auslenkung des Hockers und die Rotation der Sitzfläche. Schiebt man die Sitzfläche des Stuhles leicht vorwärts oder rückwärts, so bewegt man sich in der virtuellen Welt nach vorne oder nach hinten. Schiebt man sie leicht nach links oder rechts, bewegt man sich nach links oder rechts. Rotiert man den Sitz in die eine oder andere Richtung, dreht man sich in der virtuellen Welt so lange, bis der Stuhl wieder in die Geradeausrichtung zurückgestellt wird. Das funktioniert sowohl mit minimaler als auch mit starker Bewegung. Je weiter eine Auslenkung erfolgt, desto schneller bewegt man sich oder rotiert man. Man kann den Stuhl lediglich durch Hüftbewegungen steuern, muss daher nicht den ganzen Oberkörper einsetzen, wenn man dies nicht möchte. Man kann aber auch den ganzen Körper einsetzen und dynamisch mit dem Stuhl mitgehen.

Alle beschriebenen Bewegungen funktionieren auch in Kombination. Ein schnelles Kurven fahren, ein Sliding, entsteht durch Neigung des Stuhls um $45 \mathrm{Grad}$ nach vorne und zur Seite und gleichzeitiges Rotieren der Sitzfläche. Körperlich bewegt man die Hüfte einfach tangential zur Bewegungsrichtung, ähnlich wie beim Skifahren. Das Sliding durch die virtuelle Welt fühlt sich also ähnlich einer vergleichbaren Bewegung in der realen Welt an, bei der man durch die Fliehkraft in diese Richtung gedrückt werden würde.

6 aeris-Impulsmöbel GmbH: Der Swopper ${ }^{T M}$. 

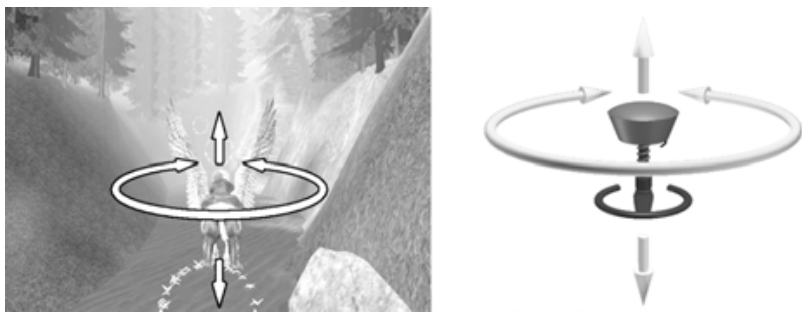

Abbildung 2: Vogelflug mit dem ChairIO. Das linke Bild zeigt die Ansicht des Spiels mit den eingezeichneten Steuerungsmöglichkeiten: Orientierung und Flügelschlag. Das rechte Bild zeigt die zugehörige Abbildung der Parameter auf das ChairIO.

\subsection{Das Erlebnis}

Neben den technischen Aspekten zeigt sich hier, wie das Erleben des Benutzers beim Steuern durch die virtuelle Welt beeinflusst wird :

- Die aktuelle Position des Stuhls wird im Sinne eines passiven Feedbacks über die eigene Körperposition zurückvermittelt an den Benutzer.

- $\quad$ Der Akteur sitzt auf dem Stuhl, kontrolliert die Bewegung des Stuhles mit seinem ganzen Körper und verschmilzt so mit Schnittstelle und Interaktion zu einem harmonischen Ganzen. Die Bewegung selbst ist harmonisch und dynamisch und wurde bereits mit Sambatanzen verglichen.

- Die Bewegung des Stuhls bringt den ganzen Körper bis in die Fußspitzen in Schwung und fördert die Beweglichkeit. Der Swopper ${ }^{\top \mathrm{M}}$ selber ermöglicht erst diese Art der Bewegung. Der Einsatz in Kombination mit dem Computer fördert und motiviert diese Bewegung dann aktiv. Statt über Stunden krumm vor dem Bildschirm des Computers zu sitzen und nur die Finger zu bewegen, wird hier der ganze Mensch aktiv gefordert und in seinem Bewegungstrieb unterstützt.

Der Benutzer verschmilzt also mit der Bewegung durch den virtuellen Raum bzw. der Steuerung der Bewegung im realen Raum. Er ist Teil der Installation, erhält direktes Feedback und hat ein Erlebnis sowohl in der virtuellen Welt in Form einer visuell vermittelten Reaktion jener Welt als auch in der realen Welt durch das passive Feedback des Stuhls und die eigene Körperposition.

Das ChairIO wurde von uns in verschiedensten Anwendungen zur Steuerung von virtuellen Welten und Spielen eingesetzt. ${ }^{7}$ Ein Beispiel, das einen Aspekt des möglichen Erlebens gut beschreiben kann, ist die Simulation eines Vogelfluges. Wir benutzten dazu eines der In-Game Spiele in „Harry Potter und der Gefangene von Azkaban ${ }^{T M}$ ، $(E A$ Games), bei dem der Nutzer normalerweise durch

7 Vgl. Beckhaus/Blom/Haringer: ChairIO - the Chair-Based Interface. 
Tastaturanschläge den Flügelschlag eines Hippogriff's und dessen Orientierung steuert. Harry, in dem Fall der Benutzer selbst, sitzt auf dem Hippogriff und soll es durch einen Ringparcour fliegen. Der Flügelschlag muss zeitlich passend ausgelöst werden, nämlich genau dann, wenn der Vogel mit dem Flügel schlagen kann. Hochfrequentes Klicken führt dabei bestenfalls zu erratischem Verhalten und geht verloren, denn solange der vorherige noch nicht abgeschlossen ist, kann kein Klick zu erneutem Flügelschlag führen. Löst man jedoch den Flügelschlag nicht aus, so gleitet der Vogel langsam sinkend vor sich hin bis zum Boden. Man muss also das richtige Timing, den richtigen Rhythmus treffen, um den Vogel in der richtigen Höhe zur rechten Zeit durch die Ringe gleiten zu lassen.

In unserer ChairIO Version, gezeigt in Abb. 2, wird ein Flügelschlag ausgelöst durch einmaliges Auf- und Abbewegen auf dem Hocker. Die Richtung kontrolliert man wieder durch leichte Rotation des Sitzes. Man bewegt sich also selbst auf und ab während der Flügel geschlagen wird. Hierdurch kommt man in eine Art Bewegungsfluss, der ähnlich dem des Vogels selber ist. Man schwingt im Gleichtakt mit dem Vogel und hat dabei das volle sensorische Feedback vom Auf und Ab, welches durch Ausbreiten der Arme noch verstärkt werden kann. Sobald man sich in dieser Weise einmal in die behäbige Flugcharakteristik des Vogels eingeschwungen hat, stellt sich ein flow-ähnliches, harmonisches, reiches Erlebnis ein, fast, als würde man selber fliegen.

\subsection{Die Benutzbarkeit}

Die Beschreibung hat am Beispiel des ChairIO's aufgezeigt, was reichhaltiges Erleben in Bezug auf Interaktion mit Computern durch bewusste Einbeziehung von realer Welt meint. Nun kann eingewendet werden, dass in unserer auf Zielerfüllung ausgerichteten Gesellschaft vor der Benutzerzufriedenheit zuerst die Funktionalität sichergestellt sein muss, also das möglichst effektive und effiziente Lösen einer Aufgabe. Die ISO 9241-11 von 1998 beschreibt die Gebrauchstauglichkeit: Das Ausmaß, in dem ein Produkt durch bestimmte Benutzer in einem bestimmten Nutzungskontext genutzt werden kann, um bestimmte Ziele effektiv, effizient und mit Zufriedenheit zu erreichen. Deshalb ist ein weiterer wichtiger Aspekt die Gebrauchstauglichkeit einer Schnittstelle in Bezug auf die Erfüllung der gestellten Aufgabe. Für das ChairIO haben wir in Benutzertests die Effektivität, Effizienz und Intuitivität der Schnittstelle überprüft und dabei nachweisen können, dass diese Schnittstelle selbst für Erstanwender sofort voll nutzbar war und diese ihre Aufgaben vollständig erledigen konnten. Ohne weitere Erklärung zur Funktionalität haben selbst Diejenigen, die zum ersten Mal auf einem ChairIO und in einer VR Welt waren, ohne Nachdenken komplexe Aufgaben gelöst, wie beispielsweise das Fahren um eine Säule bei gleichzeitigem Blick auf die Säule. Der Körper scheint intuitiv zu wissen, wie er sich bewegen muss. Das lässt sich möglicherweise so erklären, 
dass man mit dieser Art der Steuerung auf implizit bekannte Bewegungs- und Steuerungsmuster zurückgreift, beispielsweise auf das Bewegen beim Laufen oder Sitzen. Weiterhin scheint der Mensch sich gerne in diese Art der Bewegung und deren Erlebnis hineinziehen zu lassen.

\subsection{Diskussion}

Das ChairIO ist ein gutes Beispiel dafür, dass es möglich ist, sowohl präzise und intuitive Steuerungen der virtuellen Welt, als auch ein reichhaltiges Erleben der Benutzer in der realen Welt erfolgreich zu kombinieren. Die Studie und die Aussagen vieler sehr erfahrener VR Benutzer deuten sogar darauf hin, dass diese Art Interface eine der intuitivsten Möglichkeiten bei der Navigation durch 3D Welten sein könnte.

Das motiviert, weiterhin in sensorisch reichhaltige Schnittstellen zu explorieren, deren reale Komponenten nicht in den Hintergrund gedrückt sondern bewusst eingesetzt werden, da diese gerade durch ihre voll-sensorische Reichhaltigkeit und ihren Wiedererkennenswert ${ }^{8}$ ein großes Potential haben, auch intuitiv benutzbar sein zu können und dabei zusätzlich die Benutzer zufrieden oder sogar froh machen zu können.

\section{Die GranulatSynthese - Steuerung eines interaktiven Tisches}

Virtuelle Welten werden meist auf der Basis von Desktop Systemen oder immersiven Projektionssystemen diskutiert. Aktuelle Entwicklungen in der Informatik beziehen wieder verstärkt interaktive Tische ein, die bereits in den 90ern in Form der „Responsive Workbench“9 für virtuelle Realitäten existierten, inzwischen aber als monografische Multi-Touch Tische Verbreitung finden. ${ }^{10}$ Wenngleich die Möglichkeit der Interaktion mit einem TouchScreen dynamisch und intuitiv erscheint, so setzt sie dennoch den zuvor beschriebenen Trend fort, den Menschen und seine Aktivitäten nur auf Augen und Fingerspitzen zu reduzieren.

Deshalb stellt sich die Frage, wie auch bei dem Einsatz interaktiver Tische diese Reduktion überwunden werden könnte. Das im Folgenden vorgestellte künstlerische Projekt GranulatSynthese bietet dazu Ansätze.

\subsection{Die Installation}

Die „GranulatSynthese“ ist eine audio-visuelle-haptische Installation aus Vinylkörnern auf einem Rückprojektionstisch, wie in Abb. 3 zu sehen ist. Sie wurde

8 Vgl. Affordances in Gibson: The theory of affordances und Norman: Affordance, conventions, and design

9 Krüger/Fröhlich: The Responsive Workbench.

10 Han: Low-cost multi-touch. 
mit dem Eurographics John Lansdown Award for Interactive Digital Art 2007 ausgezeichnet. ${ }^{11}$ Bei diesem Interface dienen Vinylkörner als dreidimensionale Projektionsfläche und als Modelliermasse für eine aus haptischen und auditiven Komponenten bestehende Landschaft. Die Parameter zur Kontrolle des vom Computer errechneten audiovisuellen Inhalts resultieren aus der Größe und Form der offenen Tischoberfläche, d.h. aus den körnerfreien Flächen. Das Interface ist komplex in der realen und virtuellen Domäne: die Körner sind sowohl Interface mit akustischen, haptischen und spielerischen Eigenschaften als auch dreidimensionale Projektionsfläche. Zusätzlich erzeugen sie Geräusche in beiden Domänen: der vom Computer generierten und der realen. Indem der Nutzer aus dem Material heraus arbeitet, die Landschaft durch leichtes Klopfen glättet oder die Körner auf den Tisch fließen lässt, entstehen sowohl unterschiedliche reale, akustische und haptische Informationen als auch computergenerierte Geräusche, Musik und Bilder.

Das Arbeiten mit Körnern und granularem Material ist uns wohlbekannt, denn schon als Kinder haben wir Sandkästen und Strände durchwühlt und Sandkuchen, Burgen und ganze Landschaften gebaut. Der Sand eignet sich nicht nur als Baumaterial sondern bietet auch durch die haptischen Eindrücke einen speziellen Reiz. Die Eigenschaften des weich fließenden und dennoch formbaren haptischen Materials erklären die Freude am Spielen mit Sand und anderen feinkörnigen Stoffen. Es ist darüber hinaus bekannt, dass die taktile Stimulation einen positiven Einfluss auf das Lernen und die Entwicklung des Gehirns im Kindesalter und vermutlich darüber hinaus hat.

Bei der GranulatSynthese bewegt und formt der Nutzer das Material, indem er durch Anordnung von Materieansammlungen und freien Flächen eine Landschaft mit „Hügeln und Seen“ erschafft. Im Gegensatz zu der SandScape Metapher ${ }^{12}$, die den Sand zur Beschreibung einer dreidimensionalen Hügellandschaft verwendet, werden bei der GranulatSynthese Form, Größe und Position der freigelegten Tischoberfläche - also der „Seen“ - in eine Interface-Metapher integriert. Damit wird Form, Größe und Position einer freien Fläche zu einem eindeutigen, leicht reproduzierbaren Kontrollparameter zusätzlich zur Form der 3D Oberfläche.

Die Bilder unserer Installation werden von unten auf die Rückseite einer halbtransparenten Tischoberfläche projiziert. Das verwendete Vinyl-Granulat selbst ist durchsichtig, wirkt aber durch seine unterschiedliche Materialdicke bei der Modellierung der halbdurchsichtigen Oberfläche auf die von unten projizierten Bilder: freie Flächen zeigen deutlichere Bilder, wogegen bedeckte Bereiche verschwommener dargestellt werden. Die Installation erhält zusätzlich

11 Vgl. Duce: Computer Graphics Forum 26 [2007] und Beckhaus/Schröder-Kroll/ Berghoff: Back to the sandbox

12 Ishii et al.: Bringing Clay and Sand into Digital Design 
eine geheimnisvolle Komponente durch das leicht verschwommene, gefilterte, aber dennoch erkennbare Bild, sichtbar in Abb. 3 .

\subsection{Das Erlebnis}

Der Erlebnischarakter dieser Schnittstelle liegt im Ineinandergreifen von real und virtuell und seiner Kombination von akustischen, visuellen, haptischen und kinästhetischen Reizen. Das vorgestellte Interface verfügt über vielfältige sensorische Komponenten in der realen Domäne, die durch virtuelle Inhalte bereichert werden. In der realen Domäne sorgt die geschaffene Körnerlandschaft für einen visuellen Eindruck, der an japanische Zen-Felsengärten und Sandkisten aus Kindertagen erinnert. Der vom Computer erzeugte Inhalt verbindet sich mit dieser Landschaft zu einem einzigen visuell-akustischen Bereich und einer „glühenden“ Landschaft. Die echten Geräusche und der virtuelle Klangbereich vermischen sich zur Audiolandschaft und die projizierten Bilder verändern das Erscheinungsbild der realen Granulatlandschaften. Natürlich ist die Wirkung abhängig von den Eigenschaften der verwendeten Materialien. Andere Körnermaterialien, zum Beispiel Reis, verändern das besprochene Erscheinungsbild und vermitteln andere haptische Erfahrungen. Die Verwendung von duftenden Körnern, beispielsweise Kaffeebohnen ist möglich, und würde das Erlebnis um eine olfaktorische Komponente bereichern. Mit Geleekörnern könnte man sogar eine geschmackliche Komponente hinzufügen und zu einer essbaren Version der GranulatSynthese kommen. Diese Variante wäre allerdings hygienisch äußerst fragwürdig. Als Sushi Essplatz, mit angerichteten Sushi Teilen auf der gläsernen Tischplatte, wurde der Tisch jedoch bereits erfolgreich und hygienisch unbedenklich eingesetzt. Dies ist in Abb. 4 zu sehen, eine Tischprojektion ganz ohne Granulat und nur mit den visuell-auditiven und geschmacklichen Komponenten.

Der Spaß am Arbeiten mit der Granulatlandschaft ist einer der Hauptaspekte des Interface. Das Berühren der Körner ist eine höchst haptische Erfahrung und ähnelt der Berührung von Reiskörnern oder Kaffeebohnen. Auch ihre akustischen Eigenschaften sich vergleichbar. Das Material ist einerseits hart genug, um klare Geräusche zu erzeugen wenn es auf den Tisch geschüttet wird, andererseits fühlt es sich angenehm und warm an. Es animiert den Nutzer, die Hände durch die Körner wandern zu lassen und Hügel oder freie Flächen zu modellieren, was wiederum Geräusche erzeugt. Die Installation/Schnittstelle lädt zum Spielen mit den Körnern wie in einem Sandkasten ein. Tiefergehende Aspekte der Arbeit mit ihrem Potenzial als akustisches, musikalisches und bildgenerierendes Gerät können beim Ausprobieren entdeckt werden.

Das Schnittstelle stellt dem Nutzer implizite Kommunikationsmöglichkeiten mit der Installation und mit anderen Nutzern zur Verfügung. Da die Schnittstelle die gesamte Installation umfasst und nicht nur ein Benutzer ein einziges Kontrollinstrument in der Hand hält, kann jeder um den Tisch stehende Besucher 


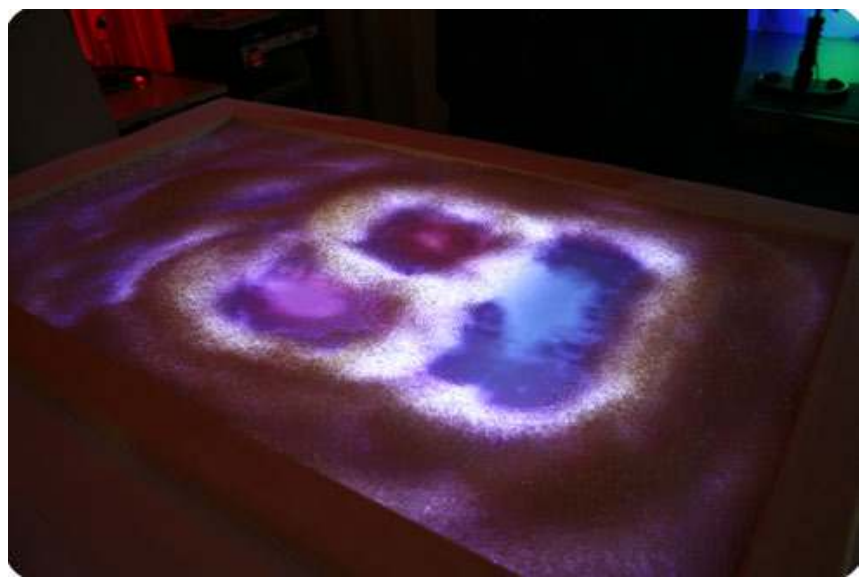

Abbildung 3: Die GranulatSynthese, eine audio-visuelle-haptische Installation, ein interaktiver musikalischer Sandkasten

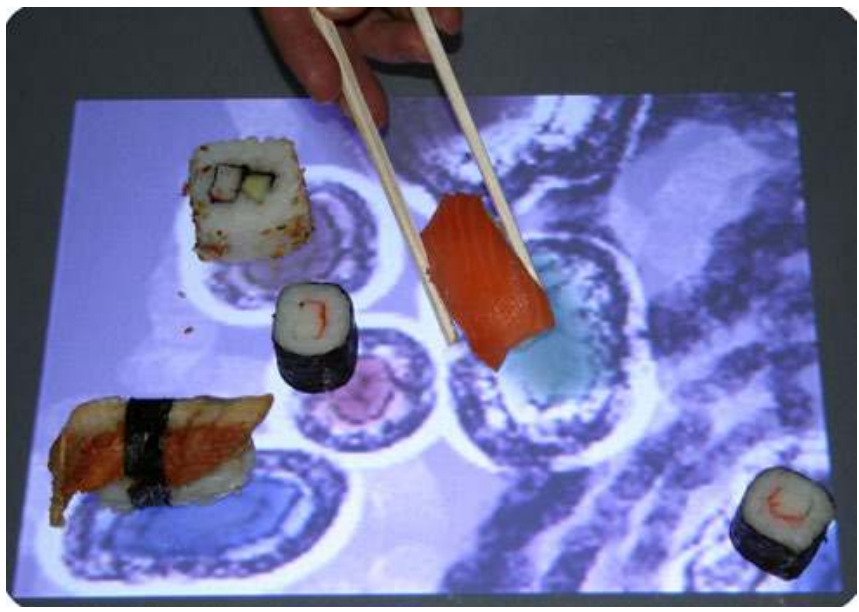

Abbildung 4: Die SushiSynthese. Ein audio-visuell-gustatorisches Erlebnis, bei dem Teile der Schnittstelle verzehrt werden können und die audio-visuelle Landschaft sich dadurch kontinuierlich verändert 
jederzeit in die Installation eingreifen und kooperativ mit den Anwesenden die Installation verändern. Die Kontrollparameter in dieser Installation sind nicht explizit und präzise dargestellt, sondern mit Intuition zu entdecken.

Benutzer treten häufig einfach zurück, um die Weiterentwicklung ihres audiovisuellen Kunstwerks oder das anderer Mitnutzer zu beobachten. Manche Besucher vertiefen sich selbstvergessen in das Spiel mit den Körnern, auch dann, wenn die Installation überhaupt nicht angeschaltet ist.

\subsection{Anwendungen und Interaktionsmetaphern}

Die GranulatSynthese-Installation bietet dem Benutzer den Rahmen zu Entdeckungen und kreativer Gestaltung. Die meisten Aspekte der jetzigen Installation sind sehr meditativ, der Besucher wird angeregt zu pausieren, um die Klangbilder auf sich wirken zu lassen. Die Installation verfügt jedoch auch erkennbar über Potenzial in weiteren Einsatzbereichen: als spielerisches und erforschendes Interface mit interaktiven Inhalten beim Geschichtenerzählen, als echtes musikund klängeproduzierendes Gerät, das sowohl physisch Klang erzeugt durch das verwendete Material als auch elektronisch durch die gesteuerten Parameter, oder als Modellierungswerkzeug von kontinuierlichen Parametern auf einer Fläche.

Die besprochene Schnittstelle wurde als künstlerische Installation entwickelt und vorgestellt, um neue Interaktionsideen auszuprobieren, deren Zugänglichkeit und Anziehungskraft zu überprüfen und um die Steuerbarkeit digitaler und kontinuierlicher Parameter zu testen. Dabei lassen sich direkt drei neue, auch allgemeingültige Interaktionsparadigmen für Tische erkennen, die mit vorherigen Schnittstellen nicht einfach möglich waren: Formbasierte, Mengenbasierte und Flächenbasierte Interaktion. ${ }^{13}$

\section{Formbasierte Interaktion}

Die Formbasierte Interaktion wurde bereits in der GranulatSynthese Installation eingesetzt. Die Form eines „Sees“ hat für den Computer eine Bedeutung. Auch Position und Größe dieser Form hat Bedeutung. Formen können vordefiniert sein oder frei umgesetzt werden. Abb. 5 zeigt sechs einfache mögliche Formen, von Schlangenlinie, über senkrechte, dreieckige, flache, runde, bis hin zur unregelmäßigen Form.

\section{Mengenbasierte Interaktion}

Bei der Mengenbasierten Interaktion werden die Regler aus der traditionellen Menüwelt über die auf ihnen liegende Menge an Granulat gesteuert. Am Beispiel eines Schiebereglers, verdeutlicht Abb. 6 das Konzept, das auf zweierlei Art

13 engl.: Shape-based, Amount-Based and Area-Based Interaction 


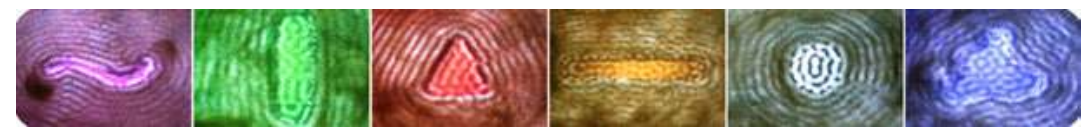

Abbildung 5: Formbasierte Interaktion: sechs Beispiele möglicher Formen

eingesetzt werden kann: der maximale Wert wird entweder bei komplett freigelegtem Regler oder bei komplett bedecktem Regler angezeigt. Die Abbildung zeigt den ersten Fall.

\section{Flächenbasierte Interaktion: Granulinge - das Spiel}

Eine besondere Ausprägung der Formbasierten Interaktion wurde in dem interaktiven Spiel „Granulinge“ auf der Tischoberfläche eingesetzt: die Flächenbasierte Interaktion. Bei dem Spiel definieren die freigelegten Flächen der Tischoberfläche die aktuelle Spielfläche und granulatbedeckte Flächen die Hindernisse.

„Granulinge“, Lemming-artige kleine Geschöpfe, müssen von ihrem Startpunkt aus über die granulatfreie Tischoberfläche zu einem vordefinierten Ziel gelangen. Sie bewegen sich stur geradeaus und ändern nur ihre Richtung, wenn sie an die Ränder der Fläche treffen. Sie werden dann wie ein Lichtstrahl auf einem Spiegel abgelenkt. Monster erscheinen unerwartet an verschiedenen Punkten der Spielfläche und fressen die dort vorbeikommenden Granulinge.

Die Benutzer gestalten das Spielfeld selbst, sodass sie den Weg der Granulinge bestimmen, die Grenzen der freien Flächen geschickt für ihre Ablenkung nutzen und einen freien Weg zum Ziel schaffen können. Gleichzeitig können diese freien Flächen jedoch auch von den Monstern erreicht werden. Die Aufgabe der Spieler ist nun das Spielfeld dynamisch so umzustrukturieren, dass die Granulinge zum Ziel gelangen, ohne von den Monstern gefressen zu werden, was man durch Ausgrenzung der Monster vom Spielfeld erreicht. Diese Art der Interaktion ist möglich, weil alle Benutzer gleichzeitig und sehr einfach an der Repräsentation des Spielfeldes arbeiten können.

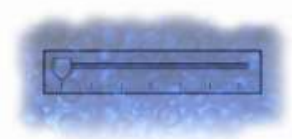

Regler 1: $0 \%$

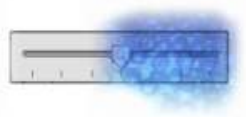

Regler 2: $50 \%$

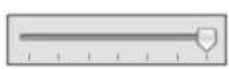

Regler 3: $100 \%$

Abbildung 6: Mengenbasierte Interaktion: Reglerwerte entsprechen dem Grad der Bedeckung 
Interessant bei diesem Spiel ist es, dass sich mehrere Spieler zusammen an dem Spiel und der Rettung der Lemminge beteiligen können. Wir haben in verschiedenen öffentlichen Demonstrationen wiederholt beobachten können, dass diese Art der Interaktion durch ihre Zugänglichkeit und Einfachheit unbeteiligte Zuschauer zu dynamischen Rettungsaktionen aktiviert hat, die zuvor passiv in zweiter Reihe standen.

\subsection{Diskussion}

Mit den hier vorgestellten ersten Anwendungen und Interaktionsparadigmen, zeigt sich die mit der GranulatSynthese Installation eingeführte Computerschnittstelle als ein Mittel, neue Möglichkeiten der Interaktion mit dem Computer zu entwickeln und neuartige Paradigmen zur intuitiven Kontrolle von Parametern eines Computers einzusetzen. Die hier vorgestellten Möglichkeiten haben bei Weitem nicht die Präzision, die man von klassischen Eingabemöglichkeiten gewohnt ist, dennoch bieten sie neue Dimensionen in der Art wie Parameter kontrolliert und erlebt werden können. Sie bringen die Steuerung des Computers in den realen Raum und geben den aktuellen Entwicklungen im Bereich der taktilen, be-greifbaren Schnittstellen ${ }^{14}$ neue Impulse.

\section{$4 \quad$ Fazit}

Computerschnittstellen sind üblicherweise so konzipiert, dass sie hinter der zu erfüllenden Aufgabe verschwinden. Sie sollen die Handlungen der Nutzer innerhalb der digitalen Domäne unterstützen, indem diese weder erkannt noch überhaupt als Teil der Anwendung gesehen werden. Die Forschung fokussiert auf eine Entwicklung rein virtueller Welten und auf eine Steuerung durch funktionale, aber stark reduzierte Eingabegeräte.

Aus der Perspektive des Menschen schlagen wir vor, reichhaltigere Abbildungsmöglichkeiten der virtuellen Welt zur Verfügung zu stellen und die reale Welt stärker und aktiv in die Schnittstelle zur virtuellen Welt einzubeziehen. Jüngste Fortschritte in der Mensch-Computer-Interaktion haben den Interaktionsraum durch die Integration von Eingabe und Ausgabe in die Domäne bereichert. In Augmented Reality-, Table Top- und Tangible InterfaceAnsätzen gehen die visuelle Projektionsoberfläche und der Interaktionsraum ineinander über, indem sie eine enge Bindung miteinander eingehen. Statt die virtuelle Welt durch ein Schlüsselloch zu betrachten, ermöglichen solche, die multisensorischen Eigenschaften realer Objekte dafür zu nutzen, dem Menschen wieder ein reichhaltigeres und freudvolleres Erleben in seiner täglichen

14 Vgl. auch die Diskussion neuer Interaktionsmodelle am Beispiel der tangible user interfaces in Ullmer/Ishii: tangible user interfaces. 
Arbeit und im Erfahren virtueller Welten zu ermöglichen. Wir glauben, dass sich durch diesen Ansatz gleichzeitig viele neue Möglichkeiten für höchst intuitive Mensch-Computer-Schnittstellen automatisch ergeben werden, eine der Grundvoraussetzungen dafür, dass virtuelle Welten überhaupt in Museen und in öffentlichen unbetreuten Installationen eingesetzt werden können.

\section{Danksagung}

Die Entwicklung der hier beschriebenen Projekte ist nur durch das Zusammenwirken verschiedener Personen möglich gewesen, denen ich sehr dafür danken möchte: Kristopher J. Blom, Matthias Haringer, Roland Schröder-Kroll, Martin Berghoff und ebenso den Studierenden der „Interaktive Medien“ Veranstaltung 2007/2008, in der neue mögliche Interaktionsmetaphern mit der GranulatSynthese untersucht wurden und das „Granulinge“ Spiel entwickelt wurde.

\section{Literatur}

aeris-Impulsmöbel GmbH: Der Swopper ${ }^{\top M}$, http://www.aeris.de letzter Zugriff: Dez, 2008.

Beckhaus, Steffi/Blom, Kristopher J./Haringer, Matthias: A new gaming device and interaction method for a First-Person- Shooter, in: Computer Science and Magic 2005, GC Developer Science Track, Leipzig, Germany 2005.

Beckhaus, Steffi/Blom, Kristopher James/Haringer, Matthias; Carsten Röcker, Carsten Magerkurth und (Hrsg.): Kap. 10 In ChairIO - the Chair-Based Interface, Shaker 2007, 231-264.

Beckhaus, Steffi/Schröder-Kroll, Roland/Berghoff, Martin: Back to the sandbox: playful interaction with granules landscapes, in: TEI '08: Proceedings of the 2nd international conference on Tangible and embedded interaction, New York, NY, USA: ACM 2008, ISBN 978-1-60558-004-3, 141-144.

Duce, David: The John Lansdown Award 2007, in: Computer Graphics Forum, 26 2007, Nr. 4, 858-859.

Gibson, James J.; Shaw, Robert/Bransford, John (Hrsg.): The theory of affordances, Hillsdale, N.J.: Erlbaum 1977, 67-82.

Han, Jefferson Y.: Low-cost multi-touch sensing through frustrated total internal reflection, in: UIST '05: Proceedings of the 18th annual ACM symposium on User interface software and technology, New York, NY, USA: ACM 2005, ISBN 1-59593-271-2, 115-118. 
Ishii, Hiroshi et al.: Bringing Clay and Sand into Digital Design - Continuous Tangible user Interfaces, in: BT Technology Journal, 22 2004, Nr. 4, 287-299, ISSN 1358-3948.

Krüger, Wolfgang/Fröhlich, Bernd: The Responsive Workbench, in: IEEE Computer Graphics and Applications, 14 1994, Nr. 3, 12-15, ISSN 02721716.

Norman, Donald A.: Affordance, conventions, and design, in: interactions, 6 1999, Nr. 3, 38-43, ISSN 1072-5520.

Rubin, Jeffrey; Hudson, Theresa (Hrsg.): Handbook of Usability Testing: How to Plan, Design, and Conduct Effective Tests, New York, NY, USA: John Wiley \& Sons, Inc. 1994, ISBN 0471594032.

Ullmer, Brygg/Ishii, Hiroshi: Emerging frameworks for tangible user interfaces, in: IBM Systems Journal, 39 2000, Nr. 3-4, 915-931, ISSN 0018-8670. 



\section{Mario Doulis/Doris Agotai/Hans Peter Wyss}

\section{Spatial Interface. \\ Wahrnehmungsfelder und Gestaltungsansätze im Virtuellen Raum}

\section{Zum Begriff „Spatial Interface“}

Die Diskussion über virtuelle Welten - über technologische Bestrebungen einerseits, künstlich erzeugte Information der physiologischen Disposition unseres Wahrnehmungsapparats anzugleichen, sowie die inhaltliche Gestaltung des Interaktionsraums andererseits - geschieht vor dem Hintergrund eines Paradigmenwechsels, den Sybille Krämer wie folgt beschreibt: „[...] wie wir denken, wahrnehmen, kommunizieren hat immer auch Folgen für die Art und Weise, in der unsere Umwelt für uns zur Welt wird, in der sich die Vorstellung über das, was für uns wirklich ist und was Wirklichkeit heisst, ausbildet und verdichtet". ${ }^{\text {" }}$ Sie geht von der These aus, dass Medien unsere Erfahrungen, unser Denken und Kommunizieren prägen, aber auch die Bildung unserer Wirklichkeitskonzepte nachhaltig verändern. Dabei formuliert sie die Frage, wie sich unsere Wirklichkeitsvorstellungen im Zusammenhang mit der durch neue Medien eröffneten Virtualisierung neu formen.

Daran anknüpfend betrachten wir die Virtualisierung zunächst als Beziehung zweier unterschiedlich beschaffener Räume, die sich gegenseitig beeinflussen, determinieren und vermeintlich gesicherte Raumqualitäten in Frage stellen. Der virtuelle Raum artikuliert sich dabei über einen liminalen Ort, eine Schnittstelle, welche im Zusammenhang mit Virtual Reality als 3D User Interface bezeichnet wird. Dieses Interface ist unter anderem Gegenstand von Untersuchungen im Bereich Mensch-Maschine-Interaktion, in der Presence-Forschung oder im Interface Design.

Folgen wir jedoch der Frage, welche räumlichen Qualitäten über dieses Interface vermittelt, welche Rezeptionsmodalitäten durch die Interaktion geschaffen werden, führt uns dies unweigerlich zu einer Modellvorstellung, welche das Interface weniger als Stelle oder konkreten Ort, sondern vielmehr als konstituierende Dimension begreift. Diese Vorstellung reicht über die Verbindung von materiell und immateriell, von real und virtuell hinaus ${ }^{2}$ und beschreibt das Interface als Wahrnehmungskonstrukt, welches die Parameter Raum, Ort, Technologie und Erfahrung miteinander verknüpft und damit einen erweiterten Bezugsrahmen öffnet. Dieses Konzept fassen wir unter dem Begriff des „Spatial Interface“ zusammen, der erstmals in der Arbeit „Mapping the World“ genauer

1 Vgl. Krämer: Medien - Computer - Realität, S. 14.

2 Vgl. Bonsiepe: Interface - Design neu begreifen. 
erörtert wurde. ${ }^{3}$ Hierbei geht es um eine thematische Verknüpfung von Aspekten der Kartographie mit aktuellen Entwicklungen im Bereich des User Interface Design. Um das Aufzeigen räumlicher Bezüge also, die nicht nur geographische sondern insbesondere auch logische und semantische Aspekte menschlicher Lebenswelten thematisieren.

\section{Spatial Interface}

In der Post-PC Ära werden Computer- und Netzwerktechnologie zunehmend in räumliche Kontexte integriert - sei dies (1) durch 3D-Interfaces im Bereich Virtual Reality und Augmented Reality, die eine räumliche Interaktion mit Computern ermöglichen, sei dies (2) durch die Anbindung an globale Datennetze wie das Internet, oder sei dies (3) durch portable Geräte, deren Nutzung nicht mehr an einen festen Arbeitsplatz gebunden ist. Darüber hinaus werden sie Bestandteil von alltäglichen Gegenständen wie Rollläden oder Türschlössern. Und Tätigkeiten wie Telefonieren, Fotografieren, Musik hören oder das Lösen eines Billets am Fahrkartenautomat sind, genau betrachtet, Interaktionen mit „Computern“. Die realen Orte, an denen dies geschieht, werden Teil eines „räumlichen Interfaces“, das sich dadurch auszeichnet, dass es uns sehr vertraut ist, nämlich in Form unserer Alltagswelt. Der hier beschriebene Raum bildet das Basisbezugssystem des Menschen. Er ist wohl der wichtigste menschliche Erfahrungsbereich. In ihm und durch ihn orientiert sich der Mensch, verortet Objekte und stellt so seine Umweltbezüge her. Raum beschreibt die Anordnung und Ausdehnung von Menschen und gegenständlichen Körpern.

Diese „natürlichen“ Eigenschaften des physikalischen Raums stellen unserem alltäglichen Denken und Handeln eine massgebliche Grundlage zur Verfügung.

Raum darf jedoch nicht nur als physikalische Grösse gesehen werden, sondern muss auch mediale und kulturelle Raumkonzepte berücksichtigen. Deshalb stellen wir einen Raumbegriff ins Zentrum, der gekennzeichnet ist durch sowohl materielle wie auch symbolische Komponenten. Erst mit einem solchen Raumbegriff kann untersucht werden, wie der Mensch sich und seine Umwelt sinnlich erfahrbar und sinnhaft erfassbar macht. ${ }^{4}$

Das Spatial Interface wird zum Raumkonstrukt, in dem die strikte Grenze zwischen Mensch und Maschine, Alltagsgegenstand und Schnittstelle, Infrastruktur und Interaktion aufgehoben wird - sowohl technisch, als auch in der Wahrnehmung des Betrachters. Es ist interaktives Abbild von inneren und äusseren Positionen des Menschen zu und in seinem Alltag.

3 Vgl. Zwimpfer/Doulis: Mapping the World.

4 Vgl. Doulis: Raum als Interface, S. 16-17. 


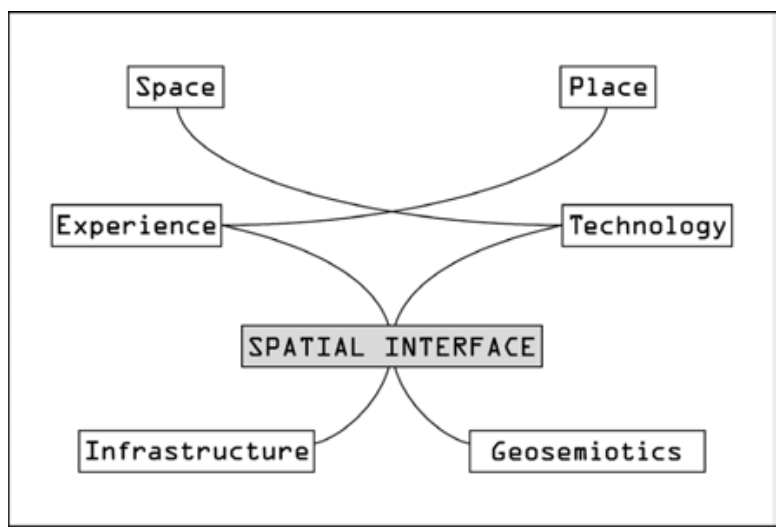

Abbildung 1: Schema des Spatial Interface und seiner raumspezifischen Merkmale ${ }^{3}$

An dieser Stelle sei erwähnt, dass, in Abgrenzung zum „Ubiquitous Computing“, das Mark Weiser in seinem Aufsatz „The Computer for the TwentyFirst Century“ beschreibt ${ }^{5}$, beim „Spatial Interface“ nicht die Integration von (Computer-) Technologie in den Alltag im Fokus der Betrachtung steht, sondern die Handlungsspielräume und -strategien, die sich daraus für den Menschen ableiten lassen. Dies zielt nicht nur auf die Nutzung des „ubiquitären Angebots“, sondern auch auf dessen Ignorierung oder gezielte Verweigerung. Die Suche nach Formen der „Sichtbarkeit technologischer Allgegenwart“ wird daher zum zentralen Thema der Gestaltung.

In einer ersten Annäherung haben wir deshalb wesentliche Aspekte des Spatial Interface zusammengetragen und einzuordnen versucht. Wir betrachten diese Aspekte und deren (teilweise komplementäre) Interdependenz auch als raumspezifische Merkmale, die sowohl auf theoretischer, als auch auf praktischer Ebene als Bezugsrahmen für die Bestimmung von Interface dienen (s. Abb. 1). Es entsteht ein flexibler Verbund von Elementen, welche aus unterschiedlichen Forschungskontexten stammen.

Im vorliegenden Modell wird „Space“ durch Technologie definiert, der mit einer äusseren oder intersubjektiven Sichtweise eine Position zugewiesen wird, welche sich geometrisch beschreiben und geographisch zuordnen lässt. Mit „Place“ hingegen wird die Betrachterposition in Bezug zum gesamten „Bezugssystem“ definiert, die den subjektiven, persönlichen Erfahrungshintergrund berücksichtigt und eine innere Sichtweise vermittelt. Die gekreuzte Verknüpfung mit den Begriffen „Experience“ und „Technology“ verweist auf die Rekombinierbarkeit der Zusammenhänge.

5 Vgl. Weiser: The Computer for the Twenty-First Century. 
Hieraus lassen sich zwei Begriffe bestimmen, die als Pole betrachtet werden können, zu denen sich alle Aspekte in Bezug setzen lassen. Während unter „Infrastructure“ in erster Linie alle technischen Aspekte zusammengefasst sind, werden unter dem Begriff „Geosemiotics“ Ansätze vereint, die realen Orten ihre Bedeutung zuweisen und Handlungsspielräume definieren, die sich nicht nur an materiell-technischen Gegebenheiten orientieren, sondern sich unmittelbar über den soziokulturellen Kontext definieren. ${ }^{6}$

Ist im ersten Fall die materielle Welt als konkretes Bezugssystem zu verstehen, hat sie im zweiten Fall eine bedeutungsgenerierende Ebene, welche visuelle, interaktionsbedingte und ortsspezifische Zuordnungen vornimmt.

\section{Virtuelle Welten als Basistechnologie}

Bei der Annahme, dass Virtuelle Welten als Basistechnologie für den Einsatz im Bereich von Kunst und Kultur zur Verfügung stehen, wird die Gesamtkonstellation als Basistechnologie verstanden - das heißt als Technologie, deren Entwicklungspotential weitgehend ausgeschöpft und in der Reifephase angelangt ist. ${ }^{7}$ Davon kann bei Virtual Reality (VR) jedoch derzeit nicht ausgegangen werden. Nach wie vor werden VR-Installationen in unterschiedlichen Konfigurationen erprobt, sei dies in Bezug auf Hardwaresettings, Eingabegeräte und Interaktionstechniken oder sei dies bezüglich der Softwaresysteme und -applikationen sowie deren Anbindungen an Netzwerke oder dynamische Datenbanken. Trotzdem stellt sich im Bereich der Einzelkomponenten sehr wohl eine Standardisierung ein. Um diesem Sachverhalt gerecht zu werden, richtet sich unsere Forschungstätigkeit unter anderem nach der bestmöglichen Modularisierung der einzelnen Komponenten.

Das Projekt „4DIVE“ 8 des Instituts für 4D-Technologien i4Ds der Fachhochschule Nordwestschweiz, welches sich mit der Visualisierung von dynamischen Prozessen in der Bauindustrie beschäftigt und Methoden entwickelt, um raum-zeitliche Prozesse aufzuzeichnen und abzuspielen, zeigt an einem praktischen Beispiel, wie eine solche Modularisierung in einem angewandten Kontext umgesetzt wurde.

Ausgehend von einem 3D-Modell eines Bauwerks sowie zusätzlichen Planungsinformationen wurde ein so genanntes 4D-Modell erstellt, um sowohl den Planungs- als auch den eigentlichen Bauprozess zu unterstützen. Für den Einsatz in Virtual Reality wurde ein Softwaretool entwickelt, welches erlaubt, ein optimiertes, zeitlich animiertes Modell zu erstellen. Ein weiteres Tool er-

6 Vgl. Scollon/Scollon: Discourses in Place.

7 Vgl. Heinrich/Lehner: Informationsmanagement, S. 152 oder Specker: Modellierung von Informationssystemen, S. 200.

8 Vgl. Doulis et al.: 4DIVE. 

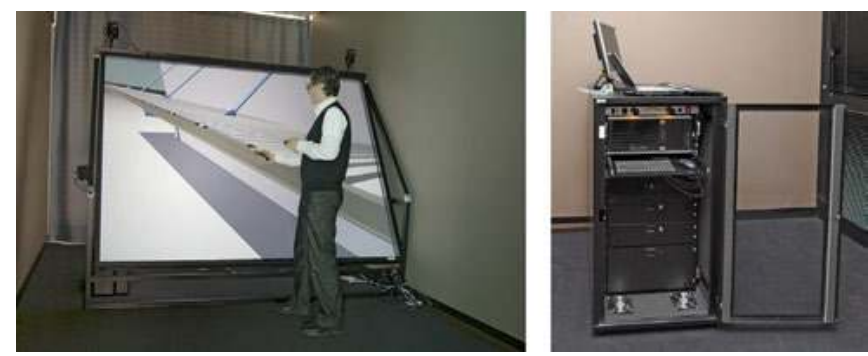

Abbildung 2: 4DIVE auf der mobilen VR-Anlage des i4Ds / 19"-Rack für die Aufbewahrung der technischen Komponenten der VR-Anlage

möglicht, die Navigation durch die räumlichen und zeitlichen Komponenten des Datenmodells als Pfade aufzuzeichnen und abzuspielen, sowie an jedem beliebigen Ort zu löschen oder neu aufzunehmen. Um eine einfache Benutzung zu gewährleisten wurden je ein Eingabegerät für die Interaktion im Virtuellen Raum sowie für das Aufzeichnen und Abspielen der räumlich-zeitlichen Pfade entwickelt.

Seitens der Software wurden modulare Komponenten mit definierten Schnittstellen implementiert, die sich an beliebige VR-Systeme sowie externe Applikationen oder Datenbanken anbinden lassen. So können sie auch in anderen Anwendungsbereichen eingesetzt werden.

Die Eingabegeräte sind modular in Bezug auf die Gehäusekonstruktion sowie die Austauschbarkeit und Rekonfigurierbarkeit aller technischer Komponenten. Dies erlaubt es Entwicklern, die Eingabegeräte einfach und kostensparend an die jeweiligen Anforderungen anzupassen oder unterschiedliche Konfigurationen zu evaluieren.

\section{VR-Studio i4Ds}

Wie bereits erwähnt, legen wir bei unseren Arbeiten grossen Wert auf die Entwicklung modularer Konzepte, die sich über einfache und stabile Schnittstellen zu komplexen Systemen unterschiedlicher Ausprägung zusammenfügen lassen.

Es lag nahe, auch unser VR-Studio anhand dieser Kriterien zu planen. Die Anlage ist daher so konzipiert, dass sie innerhalb kürzester Zeit an jeden beliebigen Ort transportiert und dort installiert werden kann. Das projektionsbasierte System verwendet Komponenten, mit denen auf den Einsatz spezieller Projektionsflächen verzichtet werden kann. Alle technischen Komponenten sind Platz sparend und transportgesichert in einem mobilen 19"-Rack untergebracht.

Für den festen Standort in unserem Labor haben wir darüber hinaus ein Experimentalsetting mit kippbarer Projektionsfläche realisiert, das es uns erlaubt, unterschiedliche Systeme einfach nachzustellen (gerade und schräge 
Wände unterschiedlicher Grösse sowie tischähnliche Systeme). Diese untersuchen wir auf ihre unterschiedlichen Qualitäten und Einsatzbereiche hin. Bei der Fertigung von Eingabegeräten greifen wir auf Rapid-Prototyping Verfahren zurück, die die Entwicklung qualitativ hochwertiger Funktionsmodelle bei kurzen Fertigungszeiten ermöglichen, was besonders bei der Erforschung neuartiger Interaktionstechniken und deren Evaluierung von zunehmender Bedeutung ist.

Unsere Entwicklungs- und Untersuchungsansätze erlauben, auf einen spezifischen Forschungsfokus einzugehen sowie schnell auf die Bedürfnisse des jeweiligen Kontexts zu reagieren.

\section{Von der Technologie zum Medium: Wahrnehmungsfelder im Virtuellen Raum}

Fassen wir Virtuelle Welten als Technologie auf, geht dieses Konzept von einer getrennten Sichtweise von technologischen und inhaltlichen Fragestellungen aus. In Anlehnung an die wechselseitige Abhängigkeit von Form und Funktion untersuchen wir jedoch, in welcher Art Inhalte durch Virtuelle Welten vermittelt werden. Dies führt uns zum Konzept des Spatial Interface, welches diese beiden Felder zusammenführt und die medialen Qualitäten in den Vordergrund stellt.

Die Ideengeschichte virtueller Kunst blickt auf eine Entstehungskette von Repräsentationsformen, Kulturtechniken und Illusionsmedien zurück, innerhalb derer Virtuelle Welten geschichtlich verortet werden können. Oliver Grau vertritt in „Virtual Art“ die These, dass Kunst in Virtuellen Welten nicht unabhängig als neues Medium betrachtet werden kann, sondern im Kontext einer Kulturgeschichte der Illusionsmedien steht. ${ }^{9}$ Er zeigt auf, wie die Herstellung von Illusionen, das Verwischen der Betrachtergrenze zum Bildraum gleichsam als anthropologische Konstante aufgefasst werden kann.

Künstlerische Ansätze reflektieren dabei entweder Virtuelle Welten als Medium oder sie hinterfragen die Technologie als Werkzeug und thematisieren deren Auswirkungen. Die Frage, welche Raumqualitäten Virtuelle Welten vermitteln, führt uns damit weg vom Technologiebegriff hin zu einem Verständnis von Virtuellen Welten als Medium, als vermittelnde Instanz, welche neue Wirkungsformen ermöglicht, neue Wahrnehmungsdispositionen von Raum schafft und damit an die eingangs zitierte These anknüpft, wie sich unsere Wirklichkeitsvorstellungen im Zusammenhang mit der durch neue Medien eröffneten Virtualisierung verändern.

Zentraler Bestandteil dieser Konstellation ist die Interaktivität, welche nicht nur ein wesentliches Merkmal dieses Mediums darstellt, sondern auch die Trennung von Inhalt und Technologie in Frage stellt. ${ }^{10}$ Interaktive Gestaltungs-

9 Vgl. Grau: Virtual Art.

10 Vgl. Tholen: Platzverweis, S. 111. 
formen konfrontieren uns mit der Frage, ob Wahrnehmung und Partizipation mit Bestandteil eines künstlerischen Werks sind. Dies schafft den Bezug zu Positionen aus der Medienkunst, ${ }^{11}$ zeigt aber auch auf, dass Inhalt und Technik sich gegenseitig bedingen. Die Interaktivität ist eng verknüpft mit dem Begriff der Immersion, die sich nun überlagert mit der Alltagswelt des Menschen. Diese Position vertreten auch Bolter und Gromala in „Windows and Mirrors“: ${ }^{12}$ Sie gehen von der Vorstellung von Fenstern und Spiegeln aus, um zu beschreiben, wie ein interaktives System beschaffen sein sollte, um eine konsistente mediale Erfahrung zu gewährleisten. Einerseits soll es dem User einen inhaltlichen Zugang ermöglichen, also transparent werden wie ein Fenster, und andererseits soll es ihm ein Gefühl der Präsenz vermitteln, indem es ihn stets mit den Inhalt in Bezug setzt, ihn also reflektiert wie ein Spiegel. Wichtig für das mediale Funktionieren eines Interfaces sei insbesondere der Rhythmus der Oszillation zwischen Fenster und Spiegelfunktion.

Wie die Brüchigkeit der Grenze zwischen diesen beiden Bereichen zum Feld künstlerischer Intervention, aber auch Gegenstand wissenschaftlicher Betrachtung wird, diskutieren Doulis und Simon in "Amalgamation“. ${ }^{33}$ Sie beziehen sich auf Fragen aus dem Produktedesign, um grundsätzliche Ansätze für die Interaktion in Virtual Reality Umgebungen aufzuzeigen. Dabei gehen die Autoren davon aus, dass diese aus einem realen Anteil - irgendeiner Art von Eingabegerät oder Aktion, z.B. einer Geste - und einem virtuellen Anteil, der Repräsentation eines spezifischen „funktionalen Verhaltens“ - eines virtuellen Objekts oder einer abstrakten Softwarefunktion - im Virtuellen Raum bestehen, die unmittelbar aufeinander Einfluss nehmen. Wichtig sei, das Verhältnis dieser Anteile zu bestimmen und auf den jeweiligen Kontext anzupassen. Dies umso mehr, da Interaktion mit und in virtuellen Welten nicht an physikalische und technische Bedingungen geknüpft ist und somit per se keinen aus dem Alltag bekannten Regeln folgen muss. Gleichwohl referenziert der Mensch in Virtual Reality stark auf seine Erfahrungen und Erwartungen in der realen Welt, was bei der Gestaltung berücksichtig werden muss. Auf diesen Erkenntnissen aufbauend wurden im Projekt SpaceActor von Studierenden des Studiengangs Industrial Design Interaktionstechniken und Eingabegeräte konzipiert und gestaltet, die den Aspekt der „Amalgamation“ untersuchten. ${ }^{14}$

11 Vgl. z.B. Gendolla/Schmitz/Schneider: Formen interaktiver Medienkunst oder Giannetti: Ästhetik des Digitalen.

12 Vgl. Bolter/Gromala: Windows and Mirrors.

13 Vgl. Doulis/Simon: The Amalgamation.

14 Vgl. Doulis et al.: SpaceActor. 

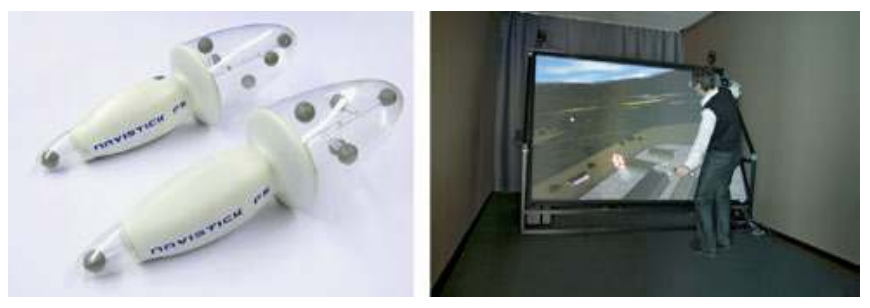

Abbildung 3: 3D-Eingabegeräte/Navigation in VR; Entwurf: Till Haldimann ${ }^{15}$

\section{Gestaltungsansätze im Virtuellen Raum}

Mit dem Erkenntnisinteresse im Bereich der angewandten Forschung nehmen wir medienimmanente Gestaltungsparameter auf und erschliessen damit ein Gestaltungspotential, welches an bestehende Konditionierungen anknüpft.

Unter dem Titel „Das unsichtbare Medium - zwischen Angleichung und Imagination. Untersuchungen zur Raumwahrnehmung in Virtuellen Umgebungen“ thematisiert eines unserer Projekte die bestehenden Forschungsbestrebungen aus dem VR-Bereich, welche darauf abzielen, eine vollständige Sinnestäuschung herbeizuführen. Es zeigt auf, wie die Verbesserung der künstlich erzeugten Information und deren Angleichung an die physiologische Disposition des Betrachters die Wahrnehmungsschwelle des Illusionsmediums möglichst tief herabsetzen soll. Die Intensität der transportierten Information wird damit maximiert, das Medium gleichsam unsichtbar.

Während die technische Entwicklung durch die VR- und Presence-Forschung vorangetrieben wird, liegt die Betrachtung der medialen Entwicklung für diesen Bereich bisher im Hintergrund. Die Ideengeschichte künstlerischer Immersionskonzepte zeigt jedoch, dass Virtuelle Umgebungen keine neuartige Erscheinung sind, sondern in der Tradition einer bildlichen Illusionsgeschichte stehen.

Mit dem vorliegenden Projekt knüpfen wir an dieses Problem an, greifen mediale Parameter auf, welche bislang abseits des technisch orientierten Diskurses standen und versuchen, diese mit Blick auf räumliche Wahrnehmungskonventionen in aktuelle 3D-Interface-Modelle einzuordnen. Wir werden Begrifflichkeiten wie Immersion, Illusion, Virtualität, Präsenz oder Aufmerksamkeit untersuchen und beobachten, wie sich Wahrnehmungsebenen dadurch verändern und welchen Stellenwert beispielsweise die Imagination innerhalb dieses Systems einnehmen kann. Viel versprechende Ansätze aus anderen medialen Bereichen wie Leerstellenfunktionen, Heterotopien, Hybridisierungen oder interaktive Narrationsstrategien können so innerhalb dieses Anwendungsfelds geprüft werden. Mit dieser Methode wollen wir ein Gestaltungspotential

15 Vgl. Doulis et al.: SpaceActor. 
erschliessen, welches neben technischen auch mediale Aspekte mit einbezieht und auf bestehende Konditionierungen zurückgreift. Neben der spezifischen Theoriebildung für den technik-, kultur- und designtheoretischen Diskurs sollen konkrete gestalterische Strategien für virtuelle Arbeitsumgebungen aufgestellt, erprobt und evaluiert werden.

\section{Resümee}

Aufbauend auf der These, dass sich unsere Wirklichkeitsvorstellungen im Zusammenhang mit der durch neue Medien eröffneten Virtualisierung verändern, spannt das Konzept des „Spatial Interface“ einen Bezugsrahmen zwischen der realen und der virtuellen Welt auf und beschreibt ein Wahrnehmungskonstrukt, welches die wechselseitige Abhängigkeit der Parameter Raum, Ort, Technologie und Erfahrung aufzeigt. Hierbei dienen uns die Begriffe „Infrastructure“ und „Geosemiotics“ als Pole, zwischen denen sich unsere Forschung bewegt.

Für den technologischen Bereich erklären die Beispiele von „4Dive“ und des Experimentalsettings des VR-Studios die Modularisierungsbestrebungen als Grundlage für die einfache Konzeption komplexer Systeme und Inhalte.

Mit dem Schritt von Technologiebegriff hin zum medialen Verständnis von Virtuellen Welten schliesslich ist die Wahrnehmung durch die Interaktivität geprägt. Welche gestaltungsrelevanten Konsequenzen sich daraus für konkrete Anwendungsszenarien ergeben, zeigt sich in unseren Ansätzen aus der angewandten Forschung, welche unterschiedliche Wirkungsbereiche des „Spatial Interface" untersuchen.

Wir versprechen uns von unserer Arbeit, dass sie technischen und künstlerischen Disziplinen gleichermassen eine Hilfestellung bietet, inhaltlich komplexe, virtuelle Erfahrungswelten zu gestalten, die einer breiteren Öffentlichkeit zugänglich gemacht werden können.

\section{Literatur}

Bolter, Jay David/Gromala, Diane (2003): Windows and Mirrors: Interaction Design, Digital Art, and the Myth of Transparency, Cambridge.

Bonsiepe, Gui (1996): Interface - Design neu begreifen, Mannheim.

Doulis, Mario (2004): Raum als Interface, Aarau: Diplomfestschrift 2004, Fachhochschule Aargau Gestaltung und Kunst.

Doulis, Mario/Simon, Andreas (2005): The Amalgamation - Product Design Aspects for the Development of Immersive Virtual Environments, in: Bullinger, A./B., Wiederhold/Meise, U./Mueller-Spahn (Hrsg.): Applied Technologies in Medicine and Neuroscience, Innsbruck, S. 51-58. 
Doulis, Mario/Vogel, Manfred/Pflüger, Jan/Rietmann, Marco/Raps, Michael (2007): 4DIVE - A 4D Interface for the Visualization of Construction Processes in a Virtual Environment, in: Proceedings of CONVR 2007, University Park PA, S. 28-39.

Doulis, Mario/Zwimpfer, Viktor/Pflüger, Jan/Simon, Andreas/Stern, Christian/Haldimann, Till/Jenni, Christoph (2006): SpaceActor - Interface Prototypes for Virtual Environments, in: Proceedings of IEEE 3DUI'06, Alexandria, S. 171-174.

Gendolla, Peter/Schmitz, Norbert M./Schneider, Irmela (2001): Formen interaktiver Medienkunst. Geschichte, Tendenzen und Utopien, Frankfurt a.M. 〈URL: http://www.medienkunstnetz.de/〉.

Giannetti, Claudia (2004): Ästhetik des Digitalen. Ein intermediärer Beitrag zu Wissenschaft, Medien- und Kunstsystemen, Wien.

Grau, Oliver (2003): Virtual Art: From Illusion to Immersion, Cambridge.

Heinrich, Lutz/Lehner, Franz (2005): Informationsmanagement. Planung, Überwachung und Steuerung der Informationsstruktur, München.

Krämer, Sybille (Hrsg.) (1997): Medien - Computer - Realität. Wirklichkeitsvorstellungen und Neue Medien, Frankfurt a. M..

Scollon, Ron/Scollon, Suzie Wong (2003): Discourses in Place. Language in the Material World, London.

Specker, Adrian (2001): Modellierung von Informationssystemen, Zürich.

Tholen, Georg Christoph (1994): Platzverweis. Unmögliche Zwischenspiele von Mensch und Maschine, in: Bolz, Norbert et al. (Hrsg.): Computer als Medium, München, S. 111-136.

Weiser, Mark (1991): The Computer for the Twenty-First Century, in: Scientific American, 265, Nr. 3, S. 94-110.

Zwimpfer, Victor/Doulis, Mario (2007): Mapping the World. Digital Qualities of Everyday Life, in: Vision Plus 12, Schwarzenberg. 


\section{Christian Spies}

\section{Zwischen Kabinett und Second Life. Bildräume und Bilderräume}

\section{Ein Blick nach Vorne}

Als die Dresdner der Gemäldegalerie im Mai des letzten Jahres auf der virtuellen Insel Dresden Gallery im Second Life auftauchte, geschah dies unter großer Beachtung und reger Berichterstattung (Abb. 1). Es war das erste Museum von internationalem Rang, das inmitten des großen Medienrummels um das Second Life eine virtuelle Dependance eröffnete; noch dazu eines, das sich als Gemäldegalerie im traditionellen Sinne versteht, die unmittelbar an seine Geschichte als höfische Kunstsammlung August des Starken anknüpft. Schließlich handelte es sich auch nicht um das Projekt irgendeines privaten oder gewerblichen Mitglieds des Second Life. Es war von den Dresdner Kunstsammlungen selbst initiiert, als Werbemaßnahme mit beachtlichen öffentlichen Mitteln vom Außenministerium und der Unterstützung der technischen Universität Dresden, von der es auch wissenschaftlich begleitet wird.

„Visit one of the world's most famous museums, the Old Masters Picture Gallery of the Dresden State Art Collections. There you can view great masterpieces of key importance for the history of art, such as Raphael's „Sistine Madonna“ or Giorgione’s „Sleeping Venus“. All the rooms of the museum have been reconstructed true to scale on the „Dresden Gallery“ island in Second Life and all 750 masterpieces in the permanent exhibition are on display."1

So lautet der Text auf der Eingangsseite zur virtuellen Galerie, für die AuBenansicht des Dresdner Zwingers und die Sempergalerie virtuell rekonstruiert worden sind (Abb. 2). Wie im realen Museum - so der Anspruch des Projekts könne man nicht nur alle Bilder an ihrem Stammplatz und im exakt nachgebildeten Rahmen sehen sondern auch ihr virtuelles Pendant mithilfe einer Audiotour durchwandern. Selbst im virtuellen Museumsshop mit Postern und Souvenirs sollen die Gewohnheiten eines üblichen Museumsbesuches nicht beeinträchtigt werden.

Bereits die ersten Einträge im offiziellen Gästebuch scheinen dieses virtuelle Museumserlebnis zu dokumentieren: „This was a nice trip to the Dresden Gallery without beeing there for real. Congratulation.“, heißt es im ersten Eintrag, „Traumhafte Architektur, wunderschöner Aufenthalt mit beeindruckender Kunst. Danke für die schönen Stunden :-)“ im zweiten. Die virtuelle Rekonstruktion der barocken Anlage des Zwingers mit Sempers Gemäldegalerie samt

1 Staatliche Kunstsammlungen Dresden. 


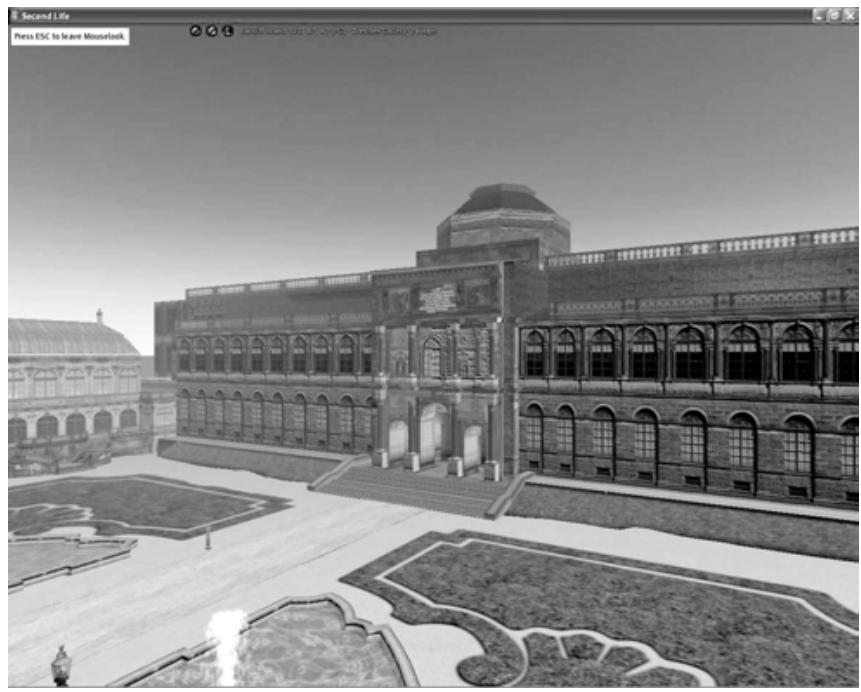

Abbildung 1: Gemäldegalerie Dresden im Second Life, Aussenansicht

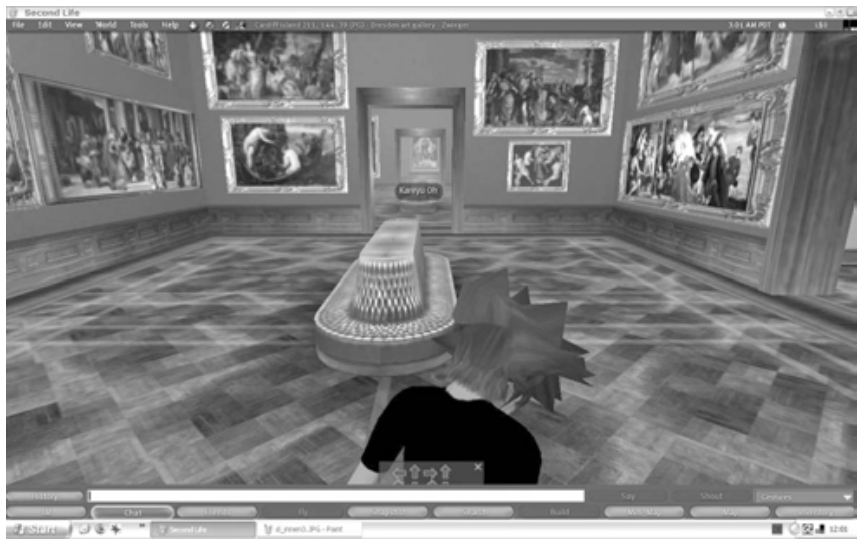

Abbildung 2: Gemäldegalerie Dresden im Second Life 
den rekonstruierten Ausstellungsräumen wird von den Besuchern tatsächlich in ihrer virtuellen Verdoppelung erfahren. Dort hält man sich auf und erlebt einen eindrücklichen Museumsbesuch, ohne tatsächlich in Dresden sein zu müssen.

Dass die reale Erfahrung der Gemälde im Galerieraum dabei in jeder Hinsicht auf die kleine Fläche des Computerdisplays reduziert wird, spielt allenfalls eine sekundäre Rolle. Dagegen wird das Second Life zum Maßstab der ,virtuellen Realität' erklärt. Denn natürlich ist man nicht so naiv, zu glauben, dass der der Besuch im Second Life tatsächlich mit dem Besuch in Dresden konkurrieren könnte. Nur zu gut kennt man die massenhaften Reproduktionen von Raffaels Sixtinischer Madonna, als dass man auf ihr Original verzichten möchte. Die bestechende Überzeugungskraft des virtuellen Museumsraums und die damit einhergehende Euphorie speist sich vielmehr durch den Kontext des Second Life, in dem die Verdoppelung der Realität per se abgesichert scheint.

Andere Kommentare im Gästebuch mögen dies abermals belegen. Die Besucher sind von der virtuellen Version der Gemäldegalerie nicht primär deshalb fasziniert, weil sie der realen Galerie so ähnlich ist, sondern weil sie sich so deutlich von den anderen Angeboten im Second Life unterscheidet. Im Unterschied zu den unzähligen Kommunikationsangeboten und Interaktionsräumen mit den oftmals mäßigen Animationen, wird die Dresdner Galerie mit der aufwendigen graphischen Umsetzung schnell als ernst zu nehmendes Angebot wahrgenommen, das sogar den Anspruch an ein vollständiges Museumserlebnis erheben kann.

Ein weiteres Argument für diese primäre Verankerung des Dresdner Projektes im Kontext des Second Life zeigt sich darin, wie schnell das Projekt mit dem nachgelassenen (Medien-)Interesse am Second Life wieder aus der öffentlichen Wahrnehmung verschwunden ist. Aller Kritik zum Trotz, die das Projekt zunächst auf den Plan gerufen hatte, scheint die Gefahr, die das virtuelle gegenüber dem realen Museumserlebnis darstellen könnte, erst gar nicht zu entstehen.

Weder das nachgelassene Interesse am Second Life, noch die kulturkritischen Bedenken gegenüber dem virtuellen Museums sollen jedoch im Folgenden mein Thema sein. Weder möchte ich hier für solche innovativen Projekte votieren, noch möchte ich in die vielfältigen kulturpessimistischen Warnungen einstimmen und ihr Misslingen vorzeichnen. Die econd Life Version der Dresdner Gemäldegalerie steht am Anfang dieser Überlegungen, da sie in einer langen Reihe von Beispielen einen der jüngsten Versuche darstellt, künstlerische Bilder durch neuartige Formen der Ausstellung auch auf ebenso neuartige Weise im Ausstellungsraum verfügbar und erfahrbar zu machen:

Im neuen Medium des Second Life, wird dabei auf eine herkömmliche Praxis des Ausstellens zurückgegriffen, um diese in die virtuelle Umgebung einzufügen. Dabei ensteht eine Differenz zwischen dem Ausstellen im vertrauten Museumsraum und in seiner virtuellen Rekonstruktion, in der die verschiedenen 
Bildebenen - vom Gemälde in der Ausstellung, über das Bild des Ausstellungsraums, bis zum virtuellen Datenraum - deutlich werden. Kurz gesagt: Einmal mehr reflektiert sich damit das Ausstellen von Bildern im eigenständigen Bild der Ausstellung, so wie dies auch bereits lange vor den virtuellen Bildern der Ausstellung der Fall gewesen ist.

\section{Ein Blick zurück}

Bereits die ersten Darstellungen der Dresdner Galerieräume im 19. Jh. (Abb. 3) oder anderer höfischer Sammlungen, die schon vorher mit den Gemälden der Liebhaber-Kabinette im 17. Jh. einsetzen, lassen sich dafür als unmittelbare Vergleichsbeispiele heranziehen. ${ }^{2}$ Zunächst könnte man anführen, dass solche Bilder in ähnlicher Weise als Werbung gedacht waren, wie auch die virtuelle Gemäldegalerie im Second Life. Sie sollten die eindrücklichen höfischen Sammlungen dokumentieren und waren als Belegstücke der feudalen Repräsentation gedacht. So kommen darin sowohl die gemalten Einblicke in die übervollen Ausstellungsräume als auch einzelne Gemälde zum Ausdruck (Abb. 4). Als Bilder der Sammlung waren sie - mit Victor Stoichita gesprochen - ,Katalog-Gemälde“, die anfangs sogar den geschrieben Katalog ersetzten. ${ }^{3}$ Der Galerieraum wird abgebildet, er wird selbst als Bild begriffen, das sich aus einer Vielzahl von Einzelbildern zusammensetzt und diese in sich vereint. Der abgebildete Raum übernimmt dabei die Funktion des Rahmens, er wird zum ,Super-Rahmen', innerhalb dessen sich ein Beziehungsnetz der einzelnen Gemälde ergibt. ${ }^{4}$ Gleichwohl muss sich darin das einzelne Bild dokumentieren. Es muss sich aus der Menge von Bildern wie die Figur aus dem Grund herauslösen lassen und den Blick des Betrachters auf sich ziehen, indem die Nachbarbilder in den Hintergrund ausgeblendet werden.

Im virtuellen Museumsraum mag ein solches selektives Fokussieren vergleichsweise einfach sein (Abb. 5, 6). Zumindest ist es in der zugrunde liegenden apparativen Struktur bereits angelegt. Der Betrachter sitzt alleine vor dem Display seines Computers und wird allenfalls von den Avataren der anderen Museumsbesucher abgelenkt. Er kann frei durch die Räume navigieren, seinen Blick konsequent steuern und mit den Zoomfunktionen des Programms jedes Bild und jeden Bildausschnitt fokussieren. An die Stelle einer festgelegten Distanz zu den Bildern und die Kontrolle durch das Museumspersonal sind nun allenfalls die Restriktionen der programmierten Parameter getreten. Damit sind die einzelnen Gemälde wie die gesamte Anordnung im Museumsraum weitgehend dem Belieben des Betrachters freigegeben.

2 Vgl. Harald Marx: Werke unsterblicher Meister aller Zeiten und Schulen, S. 7-35.

3 Victor I Stoichita: Das selbstbewusste Bild, S. 131.

4 Victor I Stoichita: Das selbstbewusste Bild, S. 125-126. 


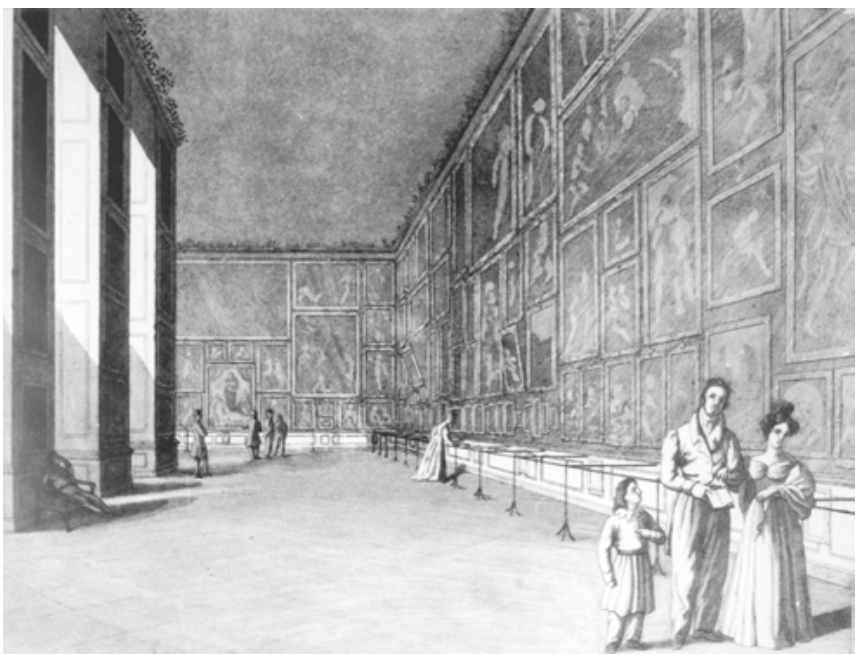

Abbildung 3: Gemäldegalerie am Jüdenhof, Dresden, um 1830

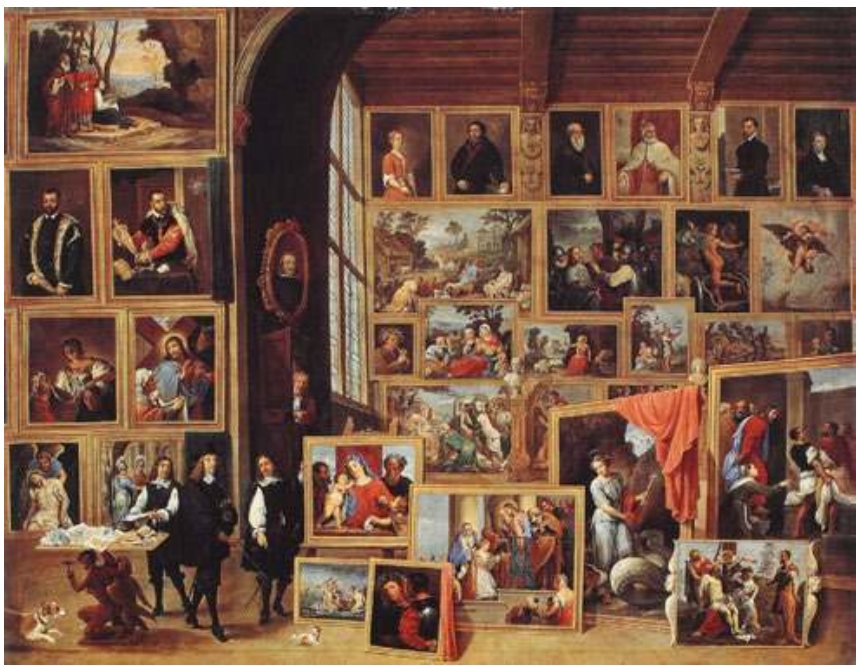

Abbildung 4: David Teniers d.J, Der Erzherzog Leopold Willhelm in seiner Gallerie in Brüssel, 1653 


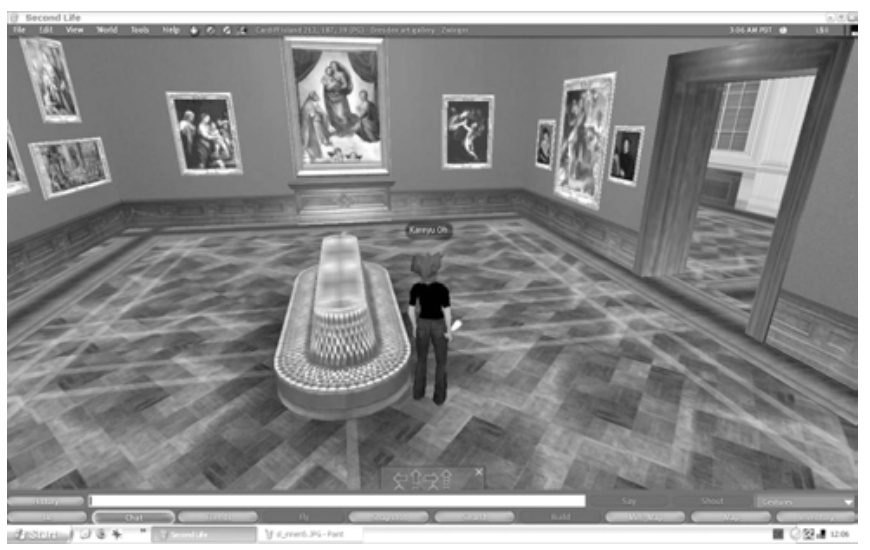

Abbildung 5: Gemäldegalerie Dresden im Second Life, Sixtinische Madonna

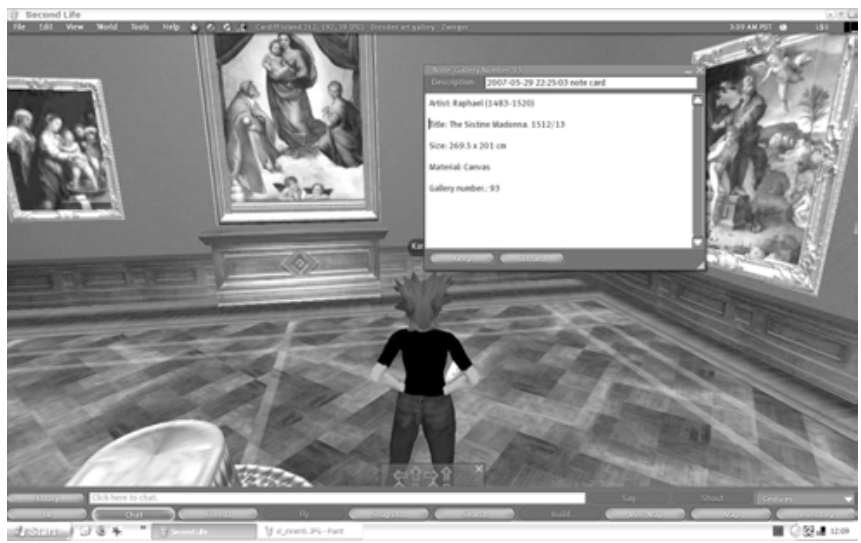

Abbildung 6: Gemäldegalerie Dresden im Second Life, Sixtinische Madonna 
In den gemalten Galeriestücken ist diese Verfügbarkeit freilich nicht gewährleistet. Es handelt sich um Abbildungen, auf denen nur Ausschnitte der Galerieräume für den Betrachter sichtbar werden. Wie bei den metallenen Abstandhaltern, die auf dem Stich der Dresdner Galerie von 1830 zu sehen sind, ist der Betrachter auf einen bestimmten Abstand zu den Gemälden festgelegt. Die einzelnen Bilder sind rasterartig auf der zweidimensionalen Bildfläche gruppiert. So entsteht eine Bilderwand, auf der die einzelnen in sich geschlossenen, transportablen Bildtafeln nun in ein dichtes Gesamtbild eingefügt werden. Nur durch die Positionierung im Zentrum oder an anderen ausgewiesenen Stellen treten einzelne Gemälde hervor. Ansonsten bleiben sie Fragmente eines umfassenden Tableaus.

Die Position des Besuchers, der sich im Second Life interaktiv durch den Museumsraum bewegt, wird im Galeriestück enorm über die dichte Bilderwand vorbestimmt. Sie zwingt zu einem Betrachten in einzelnen Fragmenten und verleitet dazu, über das einzelne Gemälde hinaus auf der zweidimensionalen Bildfläche in alle Richtungen Bezüge zu den anderen Gemälden herzustellen. Im virtuellen Museumsraum steuert der Betrachter seine linearen Bewegungen mit der Hand vor dem Computerbildschirm. Bei der gemalten Bilderwand ist er dagegen mit der Dichte von Gemälden auf der zweidimensionalen Bildfläche konfrontiert, die seine Bewegungen steuert.

Die Position im virtuellen Museumsraum, die der User über seinen Avatar steuert, kann er im gemalten Bild allenfalls über die dargestellten Staffagefiguren einnehmen (Abb. 7). Teils mag man sie als Modelle für unterschiedliche Betrachtertypen im dargestellten Galerieraum wahrnehmen. Sie fokussieren etwa mit kennerschaftlichem Blick einzelne Bilder, oder sie schreiten durch die Sammlungen, um diese nicht nur in ihrer Bandbreite zu dokumentieren sondern sich innerhalb der gezeigten Bilder auch selbst zu zeigen. Doch sind auch diese Figuren auf dem gemalten Bild festgestellt und repräsentieren einen Betrachter, der aus Sicht der aktiven User des Second Life zum passiven Gegenüber der ausgestellten Gemälde wird. Das Bild an der Wand und der Betrachter bilden zwei voneinander unabhängige Pole. Das Bild ist eine in sich abgeschlossene Einheit, der davor stehenden Betrachter eine andere.

Aus heutiger Sicht mag man dabei leicht übersehen, dass diese Vorstellung eines in sich abgeschlossenen Bildes, das der Betrachter konzentriert fokussieren kann, nicht nur für die gemalten Bilderwände des 18. Jh., sondern auch für die Besucher der höfischen Galerien nur bedingt zutrifft. In den reich dekorierten und dicht gehängten Galerieräumen des 18. Jh., die in Dresden zum Teil bis heute überdauert haben, handelt es sich vielmehr um eine Synthese von Bild und Raum, in die der Betrachter unmittelbar mit einbezogen ist (Abb. 8, 9). Dabei entsteht ein Gesamteindruck des Raums, in dem das einzelne Bild aufzugehen 


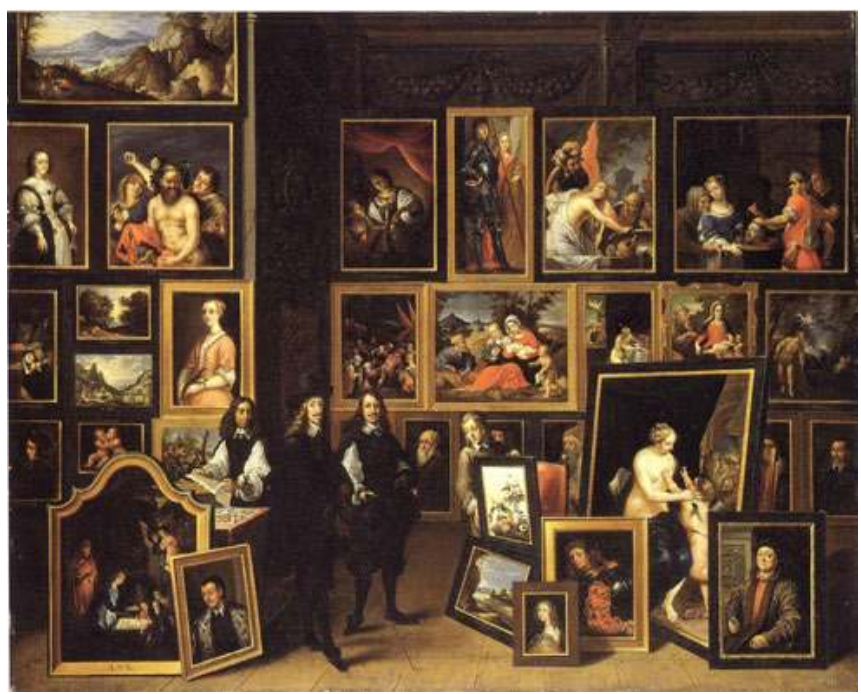

Abbildung 7: David Teniers d.J, Erzherzog Leopold Wilhelm in seiner Galerie in Brüssel, 1653

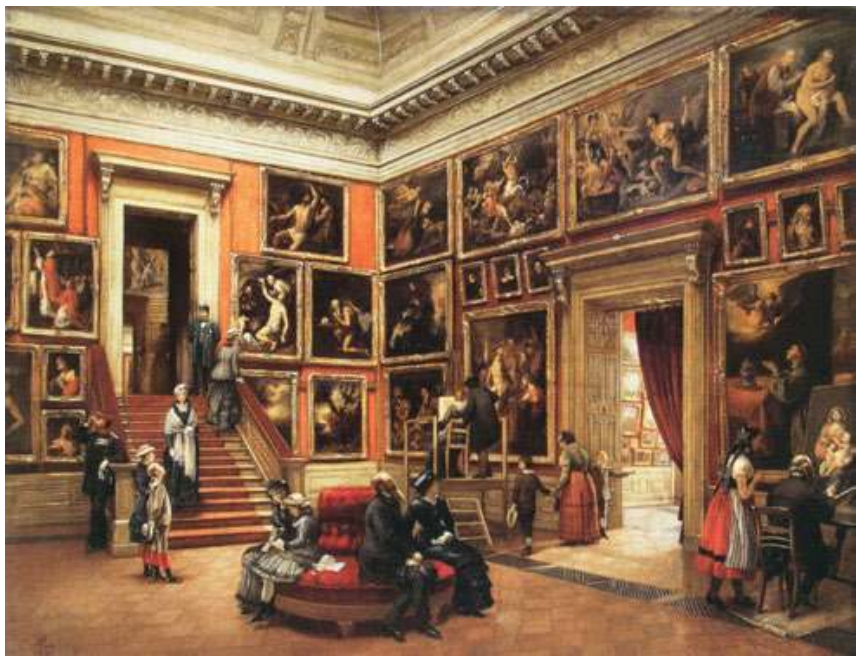

Abbildung 8: Louis Preusser, In der Dresdner Galerie, 1881 


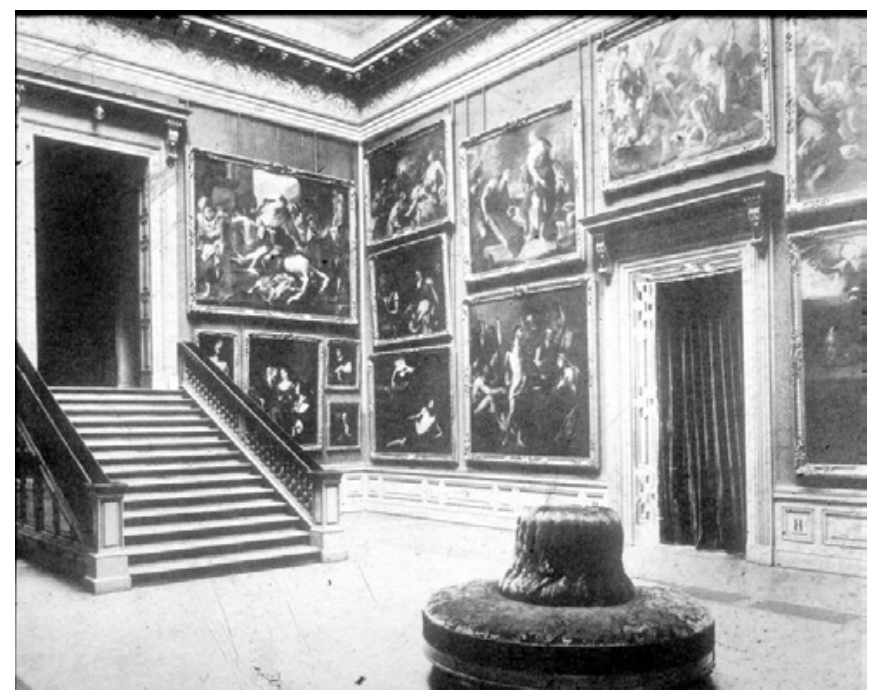

Abbildung 9: Gemäldegalerie Dresden Zustand um 1850

scheint und der sich dem Betrachter in einer Gesamtschau präsentiert. Einen solchen Eindruck beschreibt Goethe 1768 bei seinem ersten Besuch in der Dresdner Galerie, die damals noch im Johanneum untergebracht war:

Ich trat in dieses Heiligthum, und meine Verwunderung überstieg jeden Begriff, den ich mir gemacht hatte. Dieser in sich selbst wiederkehrende Saal, in welchem Pracht und Reinlichkeit bei der größten Stille herrschten, die blendenden Rahmen, alle der Zeit noch näher, in der sie verguldet wurden, der gebohnte Fußboden, die mehr von Schauenden betretenen als von Arbeitenden benutzten Räume gaben ein Gefühl von Feierlichkeit, einzig in seiner Art, das um so mehr der Empfindung ähnelte, womit man ein Gotteshaus betritt. ${ }^{5}$

Erst im Verlauf des späten 19. und 20. Jh. entwickelten sich jene neuen Ausstellungsprinzipien, die in der virtuellen Rekonstruktion mit der Synthese von Bild, Ausstellungsraum und Betrachterraum abermals umgekehrt werden. Mit der Malerei der Moderne war - vereinfacht gesagt - die Autonomisierung des Tafelbildes enorm gesteigert worden, so dass diese Autonomie nun auch bis in den Ausstellungsraum wirkte. Der abgebildete Illusionsraum wird radikal verschlossen. Das Bild selbst verdichtet sich auf seine zweidimensionale Fläche, die so auch zum Maßstab der Wand hinter dem Bild und für den gesamten

5 J. W. Goethe: Aus meinem Leben, S. 170. 


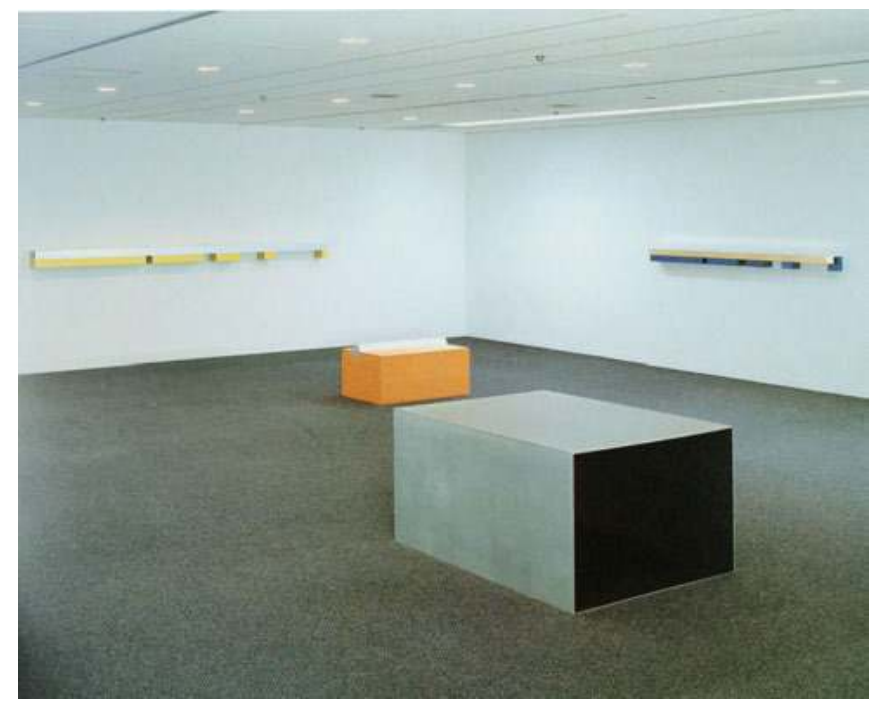

Abbildung 10: Ausstellungsansicht MOMA New York

Ausstellungsraum wird. Dabei geht es um eine mehrfache Abgrenzung, sowohl um eine Abgrenzung des Ausstellungsraums von der außerhalb liegenden Realität und noch mehr um eine Eingrenzung der Bildes auf seiner gerahmten Oberfläche. So wird das Bild nicht nur aus dem Gesamtgefüge eines dekorierten und dicht gehängten Galerieraums, sondern auch vom Betrachter losgelöst. Der ideale Raum tritt hinter das darin ausgestellte Bild zurück. Er schirmt es sogar von allem ab, was seiner autonomen Präsenz hinderlich sein könnte (Abb. 10).

Diese Entwicklung gipfelt schließlich im White Cube, dem heute bestens vertrauten leeren, weißen Galerieraum, der mit der Kunst der 1960er Jahre durchgesetzt worden ist. Mit ihm bildet sich der Topos des vermeintlich neutralen Ausstellungsraums heraus, der zum hermetischen Freiraum für das einzelne Bild oder Objekt wird. Einerseits soll dem ausgestellten Objekt damit zu seinem Recht verholfen werden. Andererseits wird genau diese weiße Kiste umso mehr zu einem sakralen Inszenierungsraum für das selbstbezügliche Kunstwerk. ${ }^{6}$ Die Feierlichkeit des Gotteshauses, von der Goethe beim Besuch der Dresdner Galerieräume spricht, liegt damit wieder unmittelbar nahe: Der

6 Diese paradoxe Umkehrung vom vermeintlich neutralen Rückzugsraum für das ausgetellte Objekt zum sakralen Inszenierungsraum ist erstmals in Michael Frieds einflussreichen Essay „Art and Objecthood“ von 1967 prägnant aufgezeigt und seitdem vielfach thematisiert worden. Vgl. Fried: Art and Objecthood, S.116147. Mit Brian O’Dohertys Essay „Inside the White Cube“ von 1973 wird dieser Ausstellungsraum dann auch historisch verortet. Vgl. O'Doherty: In der weißen Zelle. 
weiße Raum, so O’Doherty, erzeuge eine gesteigerte Präsenz, er habe „etwas von der Heiligkeit der Kirche, etwas von der Gemessenheit des Gerichtssaals, etwas vom Geheimnis des Forschungslabors“, das sich im schicken Design zu einem einzigartigen Kunstraum der Ästhetik verbinde. ${ }^{7}$

Im Unterschied zu den herkömmlichen Galerieräumen, wo die Betrachter in die Synthese des Gesamtraums eingehen, sind sie in einem solchen leeren Ausstellungsraum eigentlich gar nicht vorgesehen. Wenn bereits jeder Gebrauchsgegenstand, jeder Tisch und Stuhl, jeder Feuerlöscher oder gar jede Steckdose genauso auffallen, wie die ausgestellten Bilder und Skulpturen selbst, dann stört der Betrachter, der sich zudem noch in diesem Raum bewegt, umso mehr. Auch die Objekte selbst dürfen sich nicht gegenseitig in die Quere kommen, jedes braucht seinen eigenen Platz mit genügend Abstand zu den anderen. Der Betrachter ist, wie Brian O'Doherty schreibt, nur als Schauender willkommen, nur als körperloses Auge, das sich den Bedingungen des Bildes unterzuordnen hat. Sein raumgreifender Körper stört dagegen in diesem Ausstellungsraum, indem er in Konkurrenz zu den ausgestellten Objekten tritt und als bewegter Körper das feste Gefüge aus Raum und Bildern durchbricht. Entsprechend wird der fotografierte, menschenleere Galerieraum im installation-shot (Abb. 11) zum Paradigma dieses weißen Ausstellungsraums. So O’Doherty: „Das Bild eines weißen, idealen Raums entsteht, dass mehr als jedes einzelne Gemälde als das archetypische Bild der Kunst des 20. Jahrhunderts gelten darf.“"

Dieses fotografierte Bild der Ausstellung setzt sich nun weder aus vielen einzelnen Bildern zusammen, wie es im Galeriestück der Fall ist, noch ist es ein Bild, das der Betrachter beim Navigieren durch die virtuelle Galerie selbst herstellt. Nun stellt der weiße, hermetische Galerieraum den Rahmen dar; nicht nur für die einzelnen ausgestellten Bilder, sondern auch für das Gesamtbild, das auf diesem neutralen, weißen Grund entsteht und auf der Fotografie stillgestellt ist.

Mag man also die virtuelle Rekonstruktion der Dresdner Galerieräume im Second Life als Konkurrenz zu den Ausstellungsräumen des 19. Jh. verstehen, und mag man darin auch nur den mageren Abklatsch des realen Raum- und Bilderlebnisses sehen, dann ist sie erst recht eine Provokation für den White Cube der Moderne. Auf der zweidimensionalen Bildfläche des Monitors wird jede auratische Inszenierung im weißen Raum unweigerlich aufgelöst. Der Raum ist nun in die Obhut der User gegeben, die sich im Unterschied zu den menschenleeren Ausstellungsfotografien in Form ihrer Avatare in die virtuellen Bilder der Ausstellung einfügen.

7 O'Doherty: In der weißen Zelle, S. 9.

8 O’Doherty: In der weißen Zelle, S. 9. 


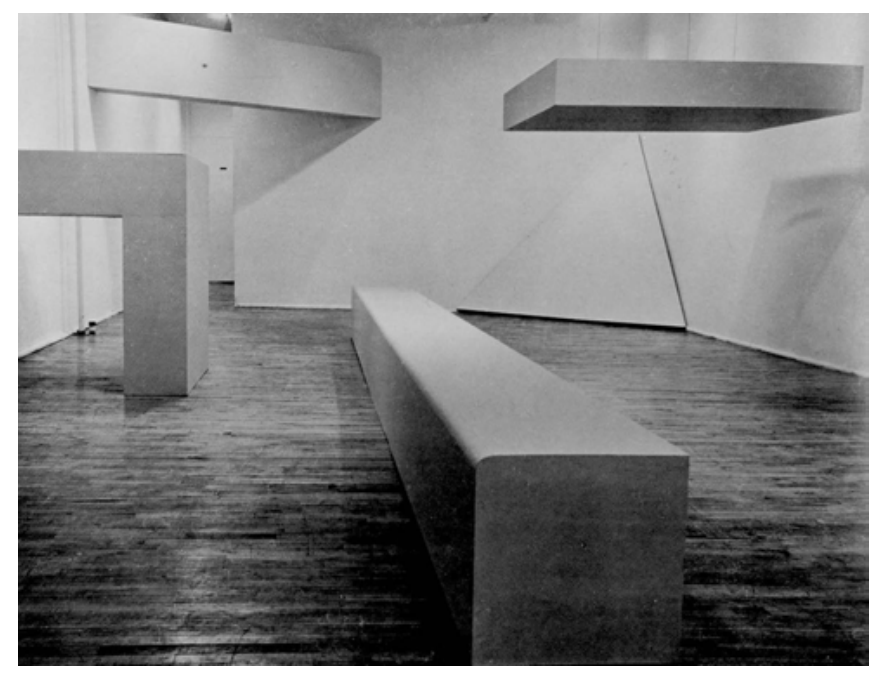

Abbildung 11: Robert Morris, Ausstellung in der Green Gallery 1964

Im Galeriestück wie im virtuellen Museumsraum werden die Bilder für den Betrachter je unterschiedlich auf neue Weise verfügbar gemacht. Über die Bildfläche des Gemäldes oder den virtuellen Raum auf dem Monitor wird er gewissermaßen an die Bilder herangeführt. Im Bild des White Cube ist dies dagegen nicht der Fall. Hier bleibt der Betrachter distanziert. Der Raum wird zum Tableau, das ihn unbeteiligt lässt und ihm allenfalls den passiven Blick von außen erlaubt. In den fotografierten, weißen Ausstellungsräumen seit den 1950er Jahren, an die wir uns mittlerweile alle so gut gewöhnt haben, erfülle sich eine Idealvorstellung, so O’Doherty. In diesem Bild der Ausstellung wird ein ,Idealraum' geschaffen, der - anderes als der reale Ausstellungsraum - auch einen idealen Betrachter fordert.

\section{Eine Alternative}

El Lissitzkys Ausstellungsräume der 1920er Jahre gehören zu keinem der drei bislang angesprochenen Typen. Vielmehr bilden sie eine Schnittstelle dazwischen, indem sie die unterschiedlichen Relationen von Bild und Betrachter zu einem Bilderraum vermitteln. Bereits bevor O'Doherty die weiße Zelle als archetypisches Bild der Kunst im 20. Jahrhundert identifizierte, hatte sich der russische Maler El Lissitzky davon verabschiedet. Einerseits stehen seine Ausstellungsräume zunächst in dieser Tradition des modernistischen Rückzugsraums. Andererseits wendet er sich jedoch davon ab, indem er zugleich auf Prinzipien der Bilderkabinette des 18. und 19. Jahrhunderts zurückgreift und dabei ein Gegenüber 


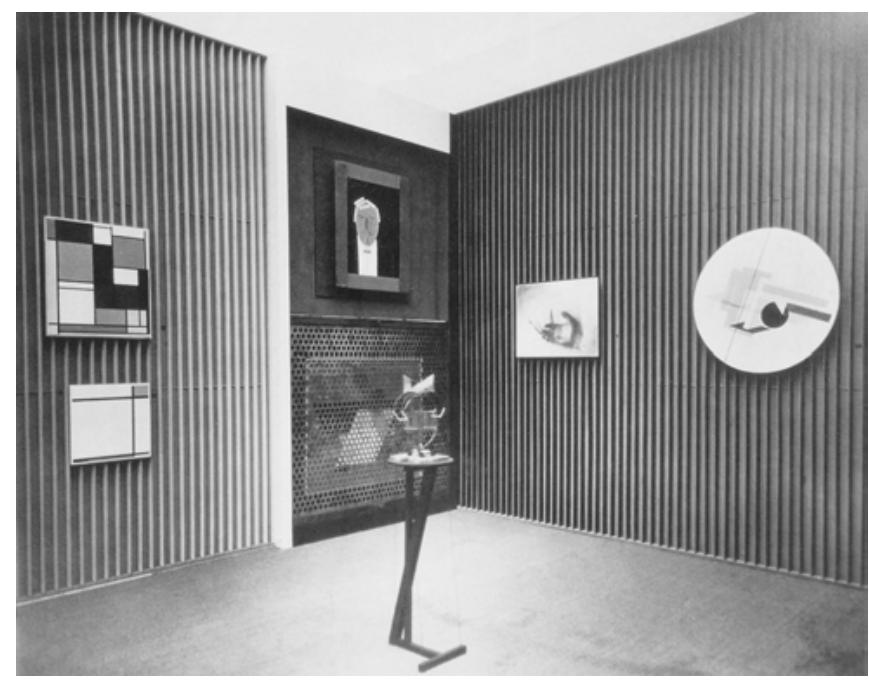

Abbildung 12: El Lissitzky, Abstraktes Kabinett, Internationale Kunstausstellung, Dresden 1926

von Raum und Betrachter entwickelt, das bereits an virtuelle Ausstellungsräume erinnert: Gemeint ist vor allem das Abstrakte Kabinett von 1926/27, das seinen Ursprung bezeichnenderweise ebenfalls in Dresden hatte (Abb. 12).

Im Rahmen der 1926 stattgefundenen Internationalen Kunstausstellung, auf der die aktuelle Kunst von Renoir bis Moholy-Nagy gezeigt wurde, gestaltete El Lissitzky einen der 56 Räume als Raum für konstruktive Kunst. Im zugehörigen Ausstellungskatalog sind lediglich die beiden Bilder verzeichnet, die Lissitzky in seinem Raum zeigte: das Runde Proun sowie das Fotogramm einer Hand. Der Raum selbst, in dem auch Bilder von Willi Baumeister, Naum Gabo, Alexei von Jawlensky, Laszlo Moholy Nagy, Oskar Schlemmer, Georg Muche, Piet Mondrian und Francis Picabia integriert waren, findet zunächst keine Beachtung. Zu weit geht er von den vertrauten Bildformen des Flächenbildes oder der Skulptur weg und wird ,nur' als Ausstellungsraum verstanden. ${ }^{9}$

Diese konventionelle Trennung von Ausstellungsraum und ausgestellten Objekten geht jedoch nicht nur an Lissitzkys Dresdner Raum sondern auch an seinen anderen künstlerischen Arbeiten denkbar weit vorbei: Lissitzky hatte seine künstlerische Laufbahn als Grafiker und Maler begonnen. Früh war er vom Suprematismus Kasimir Malewitschs beeindruckt. Doch auch diese Malerei, die mit der vorausgegangenen Tradition gebrochen hatte und selbstbewusst in dem Postulat ihrer Autonomie gründet, bleibt für ihn allenfalls eine Zwischenstation.

9 Im Vorwort des Kataloges wird Lissitzky lediglich für die „Ausgestaltung eines Raumes für konstruktivistische Kunst“ gedankt. 


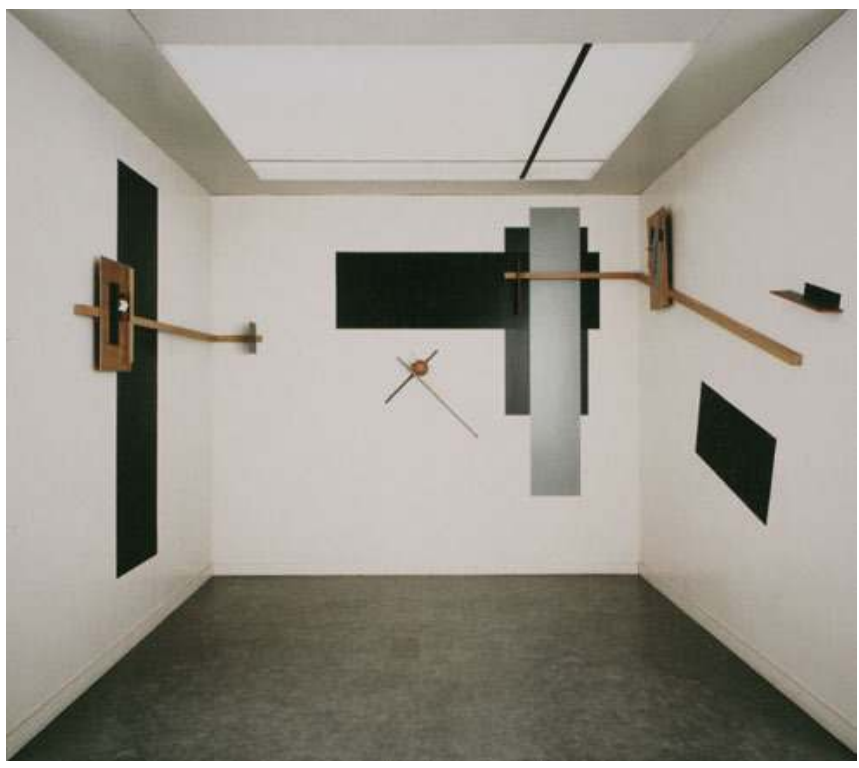

Abbildung 13: El Lissitzky, Proun Raum, Berlin 1923

Malewitschs Schwarzes Quadrat, die Ikone eines ,letzten Bildes', beschreibt Lissitzky als Platte, die „den engen Kanal der malerischen Kultur verschlossen hat." Doch sei damit keinesfalls ein definitiver Endpunkt gesetzt. Die Rückseite dieser Platte erweist sich für ihn zugleich als „mächtiges Fundament“ für eine neue Entwicklungslinie. ${ }^{10}$ Alle Splitter der Bildtradition, die zunächst noch darauf zu sehen waren, habe der Suprematismus mit seinen weißen Grundflächen hinweggefegt, um darauf den Weg in die Unendlichkeit einer neuen ,materiellen Kultur $^{6}$ freizuräumen. ${ }^{11}$

Lissitzkys Malerei dieser Zeit markiert diese neue Perspektive, von der er selber spricht. Er möchte nicht bei der zweidimensionalen Bildfläche stehen bleiben, die Malewitschs schwarzes Quadrat so demonstrativ vor Augen geführt hatte. Vielmehr möchte er sie von dort wieder in einem Raum öffnen. Dabei geht es jedoch nicht um einen herkömmlichen, zentralperspektivischen Bildraum, der durch die Bildfläche hindurch nach hinten geöffnet wird, sondern um einen realen Raum, der auf dieser Bildfläche nach vorne auf den Betrachter zukommt. Die die geometrischen Flächenformen des Suprematismus werden wieder zu dreidimensionalen Elementen, zwischen denen jedoch keine einheitliche zentralperspektivische Ansicht möglich ist. Auf ganz neue Weise wird das Bild nun zu einem ,Anschauungsobjekt': „Wir sahen, dass die Oberfläche der Leinwand

10 El Lissitzky: Proun, S. 25.

11 El Lissitzky: Der Suprematismus des Schöpferischen, S. 18. 
aufgehört hatte, ein Bild zu sein, vielmehr zu einem Gebäude wurde, das man wie ein Haus umschreiten, von oben betrachten und von unten untersuchen musste. Die einzige zum Horizont senkrecht stehende Bildachse erwies sich als zerstört. Wir haben die Leinwand zum kreisen gebracht und während wir sie drehen, schrauben wir uns selbst in den Raum hinein. “12

Von dieser Vorstellung aus ist es freilich nur ein kleiner Schritt, die zweidimensionale Bildfläche tatsächlich zu verlassen und das Bild in den dreidimensionalen Raum zu öffnen. Dann geht es um einen neuen Raum, der - wie Lissitzky schreibt - weder am Prinzip des Flächenbildes festhalte noch zusätzliche Bilder brauche: „Wir wollen den Raum als ausgemalten Sarg für unsere lebenden Körper nicht mehr“"13 schreibt er demonstrativ am Ende seines Manifests, das im Zusammenhang mit seinem 1923 entstandenen Proun-Raum steht (Abb. 13). In diesem Jahr war Lissitzky zur Großen Berliner Kunstausstellung eingeladen worden, wo er mit seinem weißen Proun-Raum erstmals keine Bilder mehr an der Wand zeigt, sondern einen gesamten Raum gestaltet. Dieser Raum erweist sich nun tatsächlich als eine dreidimensionale Version seiner Proun-Bilder: grauer Boden, weiße Decke und Wände, an denen die geometrischen Formen samt einigen Reliefelementen grösstenteils orthogonal angeordnet sind.

Schaut man sich die Fotografien des Proun-Raums an, dann mag man zunächst an die Bilder des White Cube denken. Jedoch kann man dabei leicht übersehen, dass Lissitzky diesen Raum keineswegs als menschenleer gedacht hat. Er hat ihn nicht nur unter Einbeziehung eines darin befindlichen Betrachters konzipiert, sondern hat sich diesen Betrachter immer auch als einen bewegten Körper im Raum vorgestellt. Die Bewegung ist dabei sogar durch die Raumgestaltung selbst motiviert. ${ }^{14}$ Lissitzky kalkuliert den als zeitgenössischen Ausstellungsbesucher ein, der von Bild zu Bild schreitet und in seinem Raum von Wand zu Wand herum gehen soll. In der dreidimensionalen Version verzichtet er deshalb auf ein ursprünglich auf dem Boden geplantes graues Rechteck, das dem Betrachter einen festen Standpunkt zugewiesen hätte. ${ }^{15}$ Am deutlichsten wird diese geplante Bewegung des Betrachters sicherlich in den Raumecken, wo einzelne lineare Reliefelemente von einer auf die nächste Wand fortgeführt sind. Im Kontrast dazu bilden die kleinen Reliefs an den einzelnen Wänden Fokuspunkte aus, vor denen der Betrachter stehen bleibt. Bei alledem bleibt der Berliner ProunRaum von 1923 letztlich doch ein in sich abgeschlossener Raum, ein durch transportable Stellwände hergestelltes Kabinett, das nicht zuletzt durch seine geringen Größenverhältnisse einen eher statischen Betrachter nahe legt.

12 El Lissitzky: Proun, S. 28.

13 El Lissitzky: Prounraum., S. 361.

14 Zur Bewegung in Lissitzkys Poun Raum vgl. Osswald-Hoffmann: Zauber... und Zeigeräume, S. $265 \mathrm{ff}$.

15 Blatt 6 der 1. Kestnermappe von 1923. 
Christian Spies |Zwischen Kabinett und Second Life

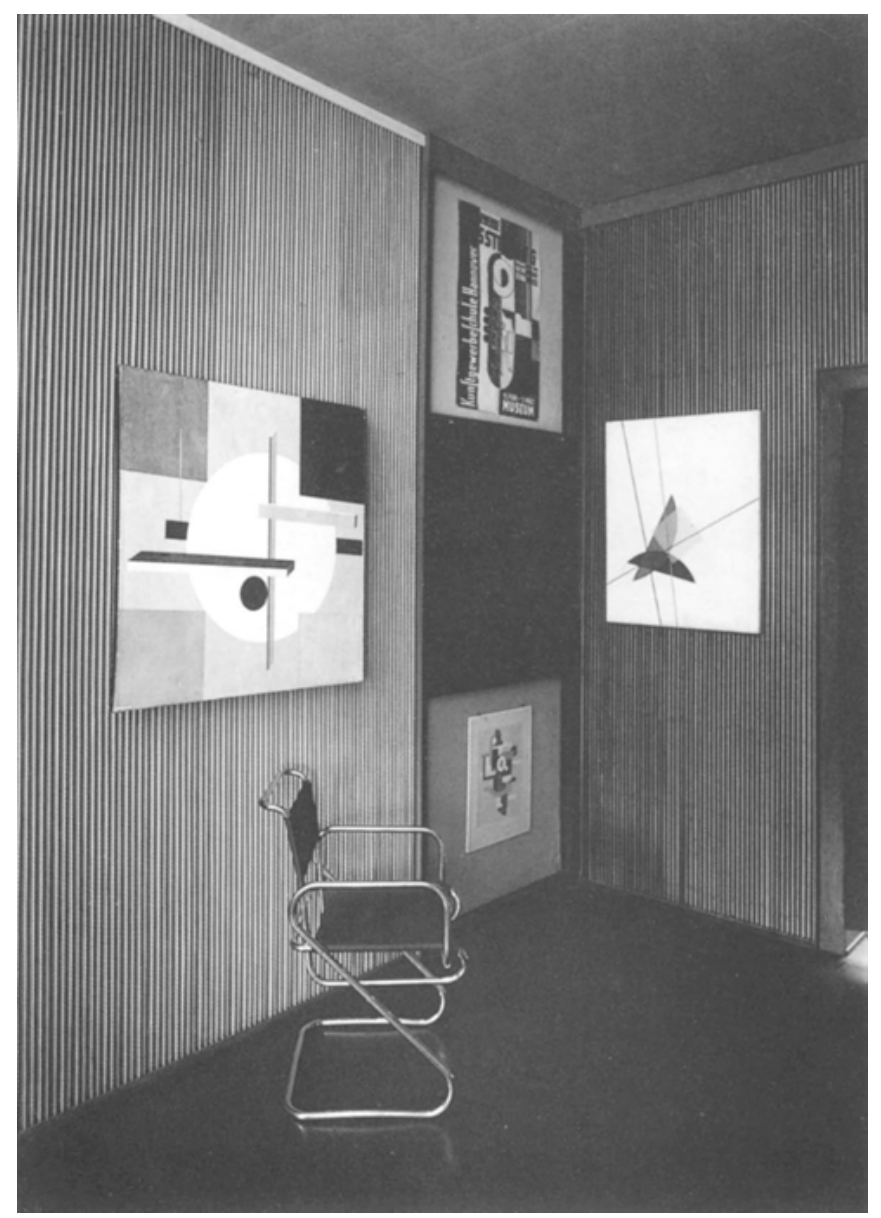

Abbildung 14: El Lissitzky, Abstraktes Kabinett, Internationale Kunstausstellung, Dresden 1926 
Christian Spies |Zwischen Kabinett und Second Life

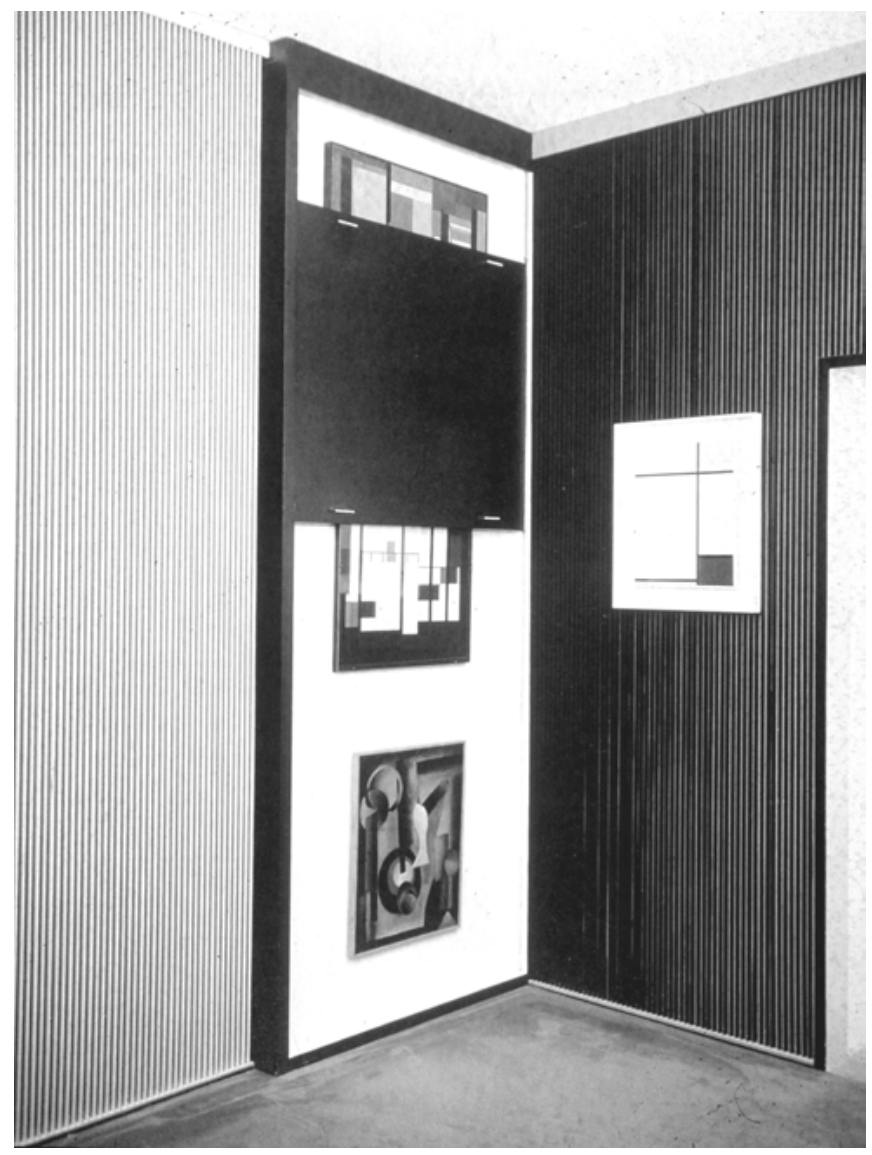

Abbildung 15: El Lissitzky, Abstraktes Kabinett, Rekonstruktion, Sprengel Museum, Hannover 
Bei dem 1926 für die Dresdner Internationale Kunstausstellung (Abb. 14, 15) entstandenen abstrakten Kabinett sind diese Begrenzungen deutlich weiter geöffnet: Der Raum ist zwar nur wenig größer, als der Berliner Raum. Er ist aber kein hermetisch abgeschlossener, selbstständiger Bildraum mehr, sondern er vereinigt Bilder unterschiedliche Künstler und mag damit zunächst einmal mehr als Ausstellungsraum unter vielen anderen erscheinen. Trotzdem bildet dieser Raum alles andere als eine neutrale, weiße Hülle für die darin ausgestellten Bilder. Die Wände sind in unterschiedliche Kompartimente gegliedert, in welche die ausgestellten Bildtafeln nahtlos eingefügt sind. Einzelne Wandfelder und Bildtafeln werden gruppiert. Dabei ergeben sich neue geometrische Formen auf den Wänden, die nun im Unterschied zum Proun-Raum nicht mehr eindeutig als Figuren auf einem Grund (Wand) gesehen werden können. Figur und Grund treten nun in ein Wechselverhältnis, so wie dies auch zwischen den Wandgliederungen und den einzelnen Gemälden der Fall ist.

Für den Betrachter, der seit den 1920er Jahren zunehmend an einen weißen, hellen Raum gewöhnt ist, mögen darüber hinaus vor allem die Farben der Wände irritieren, die in unterschiedlichen Schwarz-Weiß und Bunttönen gehalten sind. Einige Wandteile sind zudem mit einem Lamellensystem versehen, bei dem dünne Metallstreifen an die Wand montiert sind, die auf der einen Seite Weiß und auf der anderen Seite Schwarz lackiert sind. Bewegt sich der Betrachter vor diesen Lamellenstrukturen, dann verändert sich die Farbe der Wand mit jeder Bewegung. So wird die Wand als Hintergrund der Bilder, die üblicherweise einen festen Grund bildet, dynamisiert, worauf der Betrachter sogar selbst Einfluss hat.

An zwei weiteren Stellen wird diese selbstständige Rolle des Betrachters nochmals gesteigert, indem verschiebbare Lochbleche vor die ausgestellten Bildern montiert sind: Ist das Blech nach unten geschoben, liegt das obere Bild frei, ist es nach oben geschoben, bleibt das untere sichtbar. Durch die Löcher des Bleches hindurch bleiben aber auch Reste des jeweils verdeckten Bildes sichtbar, die sich abermals entsprechend der Bewegung des Betrachters verändern.

Im Unterschied zum Proun-Raum, wo der Betrachter durch die Raumgestaltung gelenkt wird und so zum bewegten Betrachter werden muss, kann der Betrachter im abstrakten Kabinett das Bild des Raumes selbst beeinflussen: Entweder greift er im direkten Sinne des Wortes ein, indem er die Lochbleche verschiebt oder aber auch indirekt, indem er sich durch den Raum hindurchbewegt. Jeweils kommt es zu einer Synthese von ausgestellten Bildern und Ausstellungsraum in einem Bildraum, in den der Betrachter als Körper sowohl integriert ist, und als Betrachtender zugleich davon distanziert bleibt.

Mit den Entwürfen für das Dresdner Kabinett lassen sich abschließend nochmals die Merkmale dieses Bilderraums zusammenfassen (Abb. 16, 17): Auf 
Christian Spies |Zwischen Kabinett und Second Life

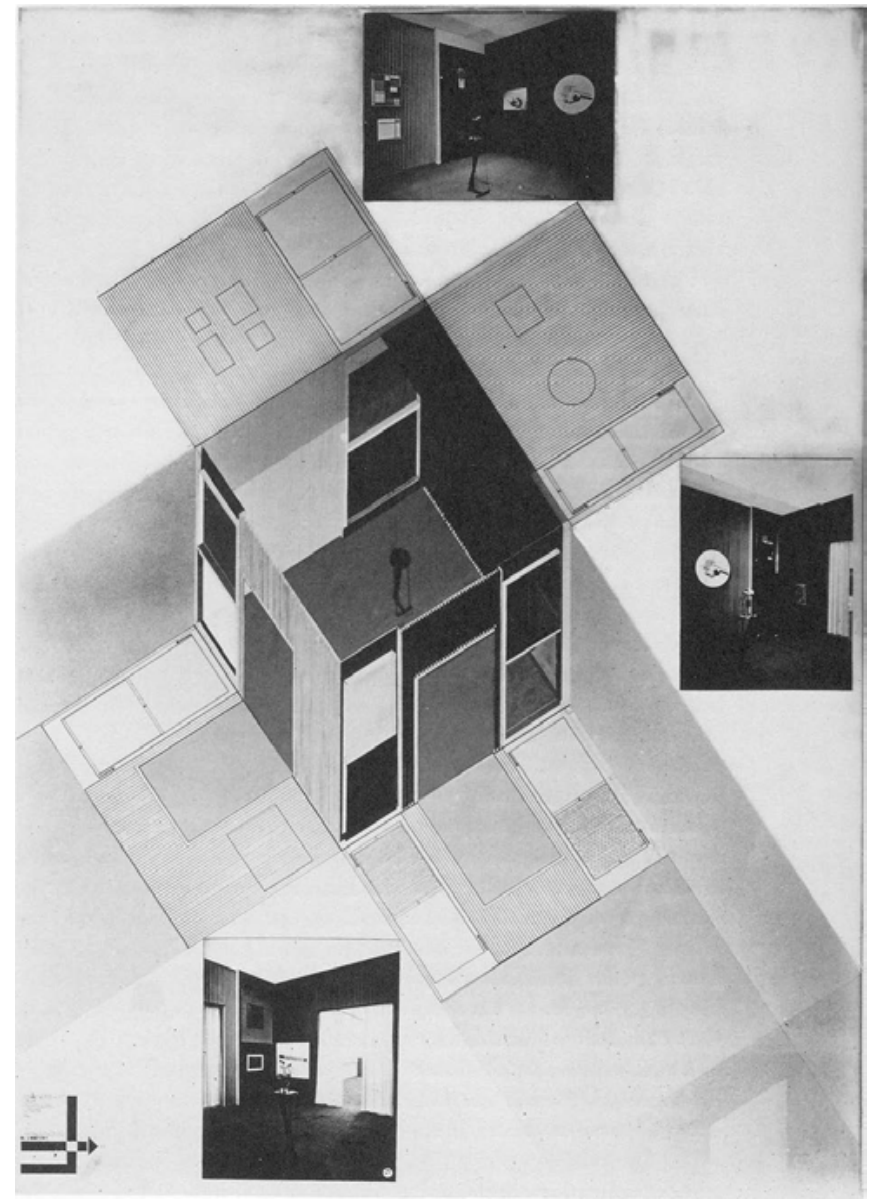

Abbildung 16: El Lissitzky, Abstraktes Kabinett, Internationale Kunstausstellung, Dresden 1926 


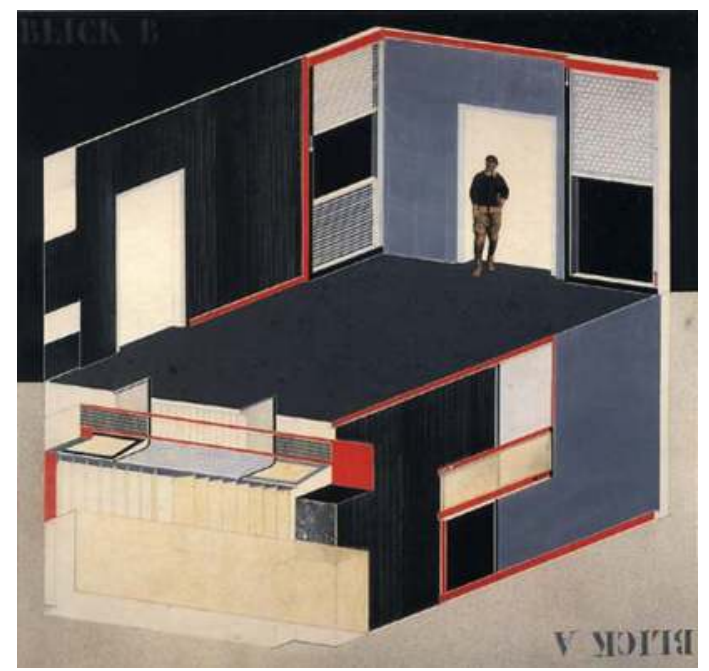

Abbildung 17: El Lissitzky, Abstraktes Kabinett, Niedersächsische Landesgalerie Hannover, Entwurf 1927

der quadratischen Entwurfzeichnung von 1927 ist der kastenartige Raum in einer axonometrischen Darstellung zu sehen. Zu dem Grundkörper aus Boden, Fensterwand und einer Längswand, die einer vertrauten Architekturzeichnung ähnlich ist, fügt Lissitzky auch die Eingangswand und die andere Längswand nach unten geklappt hinzu. So entwirft er seinen gesamten Raumkörper auf der zweidimensionalen Bildfläche. Um das Parallelogramm der grauen Bodenfläche entsteht mit den beiden Transformationen der Wände eine sechseckige Flächenform, die fest in das quadratische Bildformat eingefügt ist. Durch die weiße Bildfläche im oberen Teil und die schwarze im unteren werden die beiden Raumteile voneinander in ,Blick A` und ,Blick B` getrennt, so dass schließlich auch die Ausrichtung des Blattes variabel wird.

Auf der Bildfläche, d.h. unter den Darstellungsmodalitäten der Zweidimensionalität wird der dreidimensionale Raum so zum ,navigierbaren' Raum erklärt. Wie die zusammengesetzten Wandteile werden auch die Raumteile als Flächenformen aneinander gefügt. Erst der Betrachter kann diesen Entwurf imaginär zum dreidimensionalen Raum umformen, ganz so wie seine Position in der Entwurfzeichnung bereits markiert ist. Im Unterschied zu den schematisierten Raumelementen wird er als fotografierte Figur in die Projektionszeichnung hinein montiert. Er steht im Raum, auf der Schwelle innerhalb der Eingangstür. Er ist Teil des abstrakten Kabinetts und bleibt doch zugleich davon getrennt. Er steht inmitten des Bildes und muss sich doch als Betrachter davon distanzieren. 
Der Raum ist ein Kasten, er ist nach innen gewendet und nach außen geschlossen, so wie der White Cube ebenfalls einen geschlossenen Raum um das Kunstwerk bildet. Doch will dieser Raum bei Lissitzky alles andere als neutral sein. Er ist kein Ort der auratischen Sakralisierung, die sich demonstrativ im Bild des weißen Raums zeigt. Vielmehr will der Bilderraum mit den darin ausgestellten Bildern selbst zum Bildraum werden, zu einem Bildraum, der verschiedene Bilder und Bildelemente synthetisiert und in dem der Betrachter kein Fremdkörper ist.

Als ein solcher Bilderraum, der bereits am Beginn der Moderne eine Alternative zu dem zukünftigen Modell des White Cube bietet, wird dann auch die Vergleichbarkeit mit den anderen, den historisch zurückliegenden Ausstellungsräumen im 19. Jahrhundert, wie auch den zukünftigen, den virtuellen Museumsräumen des 21. Jh. deutlich: Bereits die Bezeichnung als Kabinett mag an die alten Bilderkabinette erinnern. Umso mehr schließt aber die lückenlose Gestaltung der Wand mit den ausgestellten Bildern und weiteren Dekorelementen an die übervollen Bilderwände der Gemäldekabinette an. Dort geht der Betrachter in der Bilderfülle der Kabinette auf. Er wird davon überwältigt, ganz so wie es die Repräsentationsfunktion der Räume verlangt.

Wie die Dresdner Galerie im Second Life schließlich zeigt, wird ein solcher Museumsraum in der digitalen Virtualisierung für den Betrachter nochmals auf eine ganz andere Weise verfügbar: Im virtuellen Raum entscheidet der Besucher eigenständig, was er sehen will und wohin er navigiert. Dabei müssen die ausgestellten Bilder und der Raum jedoch zwangsläufig auf der kleinen Fläche des Monitors zusammenfallen.

Lissitzkys abstraktes Kabinett steht zwischen diesen beiden Ausstellungsräumen. Wie bei den Dresdner Galerieräumen handelt es sich um einen Bilderraum. Dagegen wird der Betrachter jedoch nicht überwältigt, ihm wird sogar eine eigene Aktivität abverlangt. Gleichwohl ist der Raum dem Betrachter auch nicht ganz anheim gegeben, wie es im virtuellen Datenraum der Fall ist. Er bleibt ein realer Erfahrungsraum, den der Betrachter selbst zum Bildraum synthetisiert.

\section{Literatur}

Staatliche Kunstsammlungen Dresden, 〈URL: http://www.dresdengallery. com $\rangle$ - Zugriff am 10.07.09.

Fried, Michael: Art and Objecthood, in: Minimal Art: A Critical Anthology, New York: Battcock, Gregory 1968.

Goethe, J. W.: Aus meinem Leben. Dichtung und Wahrheit, 1808 - 1831.

Lissitzky, El: Prounraum. Grosse Berliner Kunstausstellung 1923, in: El Lissitzky - Maler Architekt Typograph Fotograf, Erinnerungen Briefe Schriften, Dresden: Lissitzky-Küppers, Sophie 1967. 
Lissitzky, El: Proun, in: Proun und Wolkenbügel, Dresden 1977.

Lissitzky, El: Der Suprematismus des Schöpferischen, in: Proun und Wolkenbügel, Dresden 1977.

Marx, Harald: Werke unsterblicher Meister aller Zeiten und Schulen. Die Dresdner Gemäldegalerie im Semperbau, in: Gemäldegalerie Alte Meister Dresden, Band 2, Köln: Marx, Harald 2005.

O’Doherty, Brian: In der weißen Zelle, 1996.

Osswald-Hoffmann, Cornelia: Zauber... und Zeigeräume: Raumgestaltungen der 20er und 30er Jahre, München 2003.

Stoichita, Victor I.: Das selbstbewusste Bild: Der Ursprung der Metamalerei, München 1998. 


\section{Markus Wacker/Matthias Lehmann/Ralph Stelzer}

\section{MySpace - Virtuelle Realitäten in dreidimensionalen Räumen}

\section{$1 \quad$ Einführung}

Schon seit einigen Jahren scheint es, dass Künstler den Anschluss an den technischen Fortschritt verloren haben und mit der immer schneller werdenden Entwicklung von Technologien nicht mehr Schritt halten können. Waren sie vor einigen Jahrzehnten sogar noch entscheidend am Entwicklungsprozess beteiligt, findet die Kunst im Bereich Virtuelle Realität nicht mal mehr Erwähnung. Das hier vorgestellte Projekt „MySpace“ zeigt, wie Kunst den Umgang und die Auseinandersetzung mit neuesten Entwicklungen umsetzen kann - mit der Idee, modernste Technik der Computergraphik als künstlerisches Medium einem breiteren Publikum zu öffnen, als Kunstobjekt einzusetzen und das Vorhaben mit einer Gruppe von Studenten des Studiengangs Medieninformatik der HTW Dresden zu realisieren.

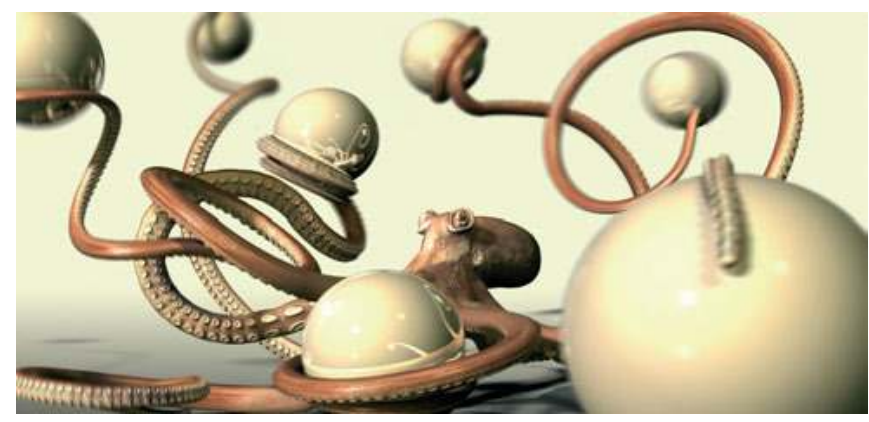

Abbildung 1: Einladung und digitaler Druck zum Myspace Projekt.

\section{Kunst und technische Medien}

Waren in den 50er und 60er Jahren des 20. Jahrhunderts Künstler durch ihre Arbeiten noch maßgeblich am Entstehungsprozess und der Weiterentwicklung technischer Medien beteiligt (beispielsweise John Cage oder Nam June Paik), so bleibt heute durch ihre zunehmende Kommerzialisierung und ihre rasante Entwicklung vielen Künstlern der Zugang zu technischen Neuerungen verschlossen. Dabei besteht von Anfang an eine wechselseitige Abhängigkeit zwischen kultu- 
reller und medientechnischer Entwicklung. ${ }^{1}$ Man kann sich des Eindrucks nicht erwehren, dass spätestens seit den frühen 90ern, nach dem Erscheinen von Netzkunst und Netzutopie, die Kunst der schnellen technischen Entwicklung hinterherhinkt. Schaut man sich beispielsweise auf den Seiten von Wikipedia ${ }^{2}$ um, so erscheinen unter den Anwendungsbereichen von „Virtueller Realität“ (VR) nur die Begriffe Industrie, Maschinenbau, Medizin und Architektur. Vom Begriff der Kunst oder Bildhauerei ist keine Spur. Dabei sollte sich gerade zeitgenössische Bildhauerei mit der Thematik „,virtueller oder digitaler Raum und dessen Realitäten und Gesetzmäßigkeiten“ auseinandersetzen.

„Virtuelle Realität“ beginnt immer größere Auswirkungen auf verschiedenste Bereiche unserer Gesellschaft zu haben. In der Soziologie wird sie neben Raum, Zeit, Sachlichkeit und Sozialität bereits als fünfte Sinn-Dimension, nämlich als „Virtuelle Dimension“ bezeichnet, welche in naher Zukunft immer nachhaltigere Folgen für Wissenschaft, Politik, Kunst, Massenmedien und selbst Erziehung haben wird. ${ }^{3}$ In einer erst kürzlich erschienenen Studie des VDI Technologiezentrums heißt es: "Der durch Virtuelle Realität (VR) angestoßene kulturelle Wandel wird vielfältig und umfassend sein [...] Erste Signale für das Entstehen einer eigenständigen Kultur mit neuen Lebensstilen zeichnen sich bereits ab. [...] Um zu klären, unter welchen Voraussetzungen VR gestaltbar ist und welche Gestaltungsinstrumente denkbar sind, ist frühzeitig eine umfassende interdisziplinär geführte Auseinandersetzung erforderlich. “4 Diese Auseinandersetzung sollte vor allem auch auf künstlerischem Sektor erfolgen, um unabhängig von wirtschaftlicher Zweckgebundenheit, Reflexionen und Visionen zu dieser neuen Medialität entwerfen zu können. Die Möglichkeiten der VR eröffnen eine Welt, in der einzelne oder alle physikalischen Gesetze außer Kraft gesetzt oder verschärft simuliert werden können. Die Nutzung der VR-Technik in Architektur, Wissenschaft oder Entwicklung und Konstruktion zeigt, wie spielerisch und ohne materiellen Einsatz Parameter variiert und neue Planungskonzepte entwickelt werden können. Dies übertragen auf die VR im Bereich der Kunst bedeutet, dass der Einzelne, also zunächst der Künstler und dann der Kunstrezipient, mehr Möglichkeiten des Ausprobierens und Experimentierens haben als in der realen Welt. Die Aufhebung physikalischer Beschränkungen ermöglicht beispielsweise auch dem körperlich Behinderten in einer virtuellen Welt als körperlich Unversehrter aufzutreten und so gegebene physiologische Beschränkungen zu überwinden und andersartige soziale Kontakte zu knüpfen. Auch die Möglichkeiten individueller Selbstdarstellung sind nicht mehr auf bestehende Äußerlichkeiten beschränkt.

1 Vgl. Frieling/Danields: Medien Kunst Interaktion, S. 8ff.

2 Wikipedia: Virtuelle Realität.

3 Vgl. Jokisch: Virtualität oder Cyberspace.

4 Zweck: Virtuelle Realität Spiel oder Kultur prägender Faktor? 
Mit dem Projekt „MySpace“ wurde eine Plattform für solche Auseinandersetzungen begonnen und den Studenten sowohl tiefere Einblicke in die technische Realisierung auf dem Gebiet der Computergraphik als auch die Möglichkeit der Anknüpfung an verschiedene Schnittstellen anderer Bereiche wie zum Beispiel Kunst und Kultur gegeben.

\section{Medientechnologie}

Seit den 90er Jahren ist die Medientechnologie zum bestimmenden Leitmotiv geworden, aus dem soziale, ökonomische und kulturelle Veränderungen hervorgegangen sind. Die Bedeutung von „Interaktivität“ ist heutzutage wesentlich an die elektronischen Medien gebunden. Die Technik des Interfaces und die Regeln der Software, in unserem Falle die der CAVE der TU Dresden, geben den Rahmen der präsentierten Interaktion in „MySpace“ vor, die zwischen Mensch und Maschine, zwischen Realraum und Datenraum stattfindet. Die Entwicklung solcher Interfaces ist wiederum seit den 90er Jahren eine der kreativsten Schnittmengen von künstlerischen und technologischen Ansätzen. Alle Techniken der Virtuellen Realität bilden eine Erweiterung der Wahrnehmung und stellen eine Verbindung der Datenstruktur mit dem Körper her. Der Betrachter erhält in unserem Falle eine neue Rolle, er tritt nicht nur als Rezipient, sondern auch als Akteur auf. Hinzu kommt, dass die interaktive Installation „My Space“ nur einen einzigen Betrachter in Aktion treten lässt, der damit einen bestimmten Platz einnimmt und somit zur Vervollständigung der Interaktion dazugehört. Von künstlerischer Seite wird der Gedanke, dass der Betrachter mit zur Installation und zum Darstellungsraum gehört, somit insgesamt zum Bildraum wird, und nicht von diesem getrennt und losgelöst betrachtet werden kann, in den Arbeiten von El Lissitzky besonders deutlich. Nähere Ausführungen sind in dem Artikel von Dr. des. Chr. Spies zu finden. ${ }^{5}$ In unserem Beispiel der MySpace-Installation in der CAVE ist der Besucher sogar nicht mehr ein Betrachter unter vielen, er tritt nicht mehr als Gruppe auf, sondern er wird zum „exemplarischen Betrachter“. Die tatsächliche Situation des „exemplarischen Betrachters“ im Ausstellungskontext ist natürlich oft alles andere als einsam. Andere Besucher sehen ihm vielleicht bei der Interaktion zu oder warten ungeduldig, bis sie selbst an der Reihe sind. Entscheidend bleibt die Einsamkeit vor dem Apparat, vor der Maschine.

\section{Die Technik der CAVE}

Seit September 2006 verfügt die TU Dresden zur Visualisierung komplexer dreidimensionaler Modelle über eine CAVE (CAVE Automatic Virtual Envi-

5 Siehe S. 65ff. 
ronment) (Abb. 2, 3 und 4). Eine CAVE ist ein abgeschlossener Raum der durch eine unterschiedliche Anzahl von Projektionswänden begrenzt wird. Auf jede dieser Wände erfolgt eine stereoskopische Projektion, die insgesamt nahezu den kompletten Sichtbereich des Betrachters abdeckt. Zusätzlich wird ständig der Standpunkt des Betrachters gemessen und daraus die korrekte Darstellung auf jede einzelne Wand berechnet. Die räumliche Darstellung ist dabei so realistisch, dass sich der Nutzer in die virtuelle Welt integriert fühlt. Die CAVE der TU Dresden hat fünf Projektionswände und gehört zu den modernsten Anlagen Europas. Neben der Projektionstechnik für anspruchsvolle dreidimensionale Modelle verfügt die CAVE zusätzlich über eine Audio-Anlage. Mit der Kombination visueller und akustischer Eindrücke wird eine verstärkte Immersion in die virtuelle Umgebung erreicht.

Als Stützkonstruktion der CAVE dient eine robuste Aluminium-Konstruktion, welche die Projektionswände und weitere technische Komponenten aufnimmt (Abb. 4). Die seitlichen Projektionswände und die Decke bestehen aus einem speziell beschichteten Acrylglas. Diese Beschichtung stellt eine homogene Lichtverteilung über die gesamte Projektionsfläche sicher und verhindert gleichzeitig störende Reflexionen in den Ecken. Die begehbare Bodenprojektionsfläche bezieht ihre Tragfähigkeit aus mehrfach miteinander verklebten Glasscheiben.

Die Projektion der Bilddaten erfolgt mit leistungsfähigen UXGA-Beamern (Auflösung 1600x1200 Pixel) der Firma Barco. Pro Wand werden jeweils zwei Beamer zur Darstellung des Bildes für das rechte bzw. linke Auge eingesetzt. Durch die Ausstattung der Beamer mit Infitec-Filtern (schmalbandigen RGBFiltern) kann eine ausgezeichnete Separation der projizierten Bilder für das jeweilige Auge realisiert werden, was ein räumliches Erleben der virtuellen Welt unabhängig von der Kopfhaltung garantiert. Die ebenfalls in Abb. 4 erkennbaren Spiegel ermöglichen die Verringerung des Abstandes zwischen Beamern und Projektionswand, indem der Lichtweg abgewinkelt wird.

Die Berechnung der projizierten Bilder erfolgt über ein PC-Cluster, welches aus elf PCs besteht. Jeder dieser PCs verfügt über 3GB Hauptspeicher und mit einer Nvidia Quadro FX4500 über ausreichende Grafikleistung. Zur Speicherung der Modelldaten dient ein Server mit 1 Terabyte Speicherkapazität. Hinzu kommt ein PC für die Bereitstellung der akustischen Signale sowie ein PC zur Verarbeitung der Positionsdaten des Nutzers.

Der Innenraum der CAVE wird mit Infrarotkameras aufgenommen, die in den Ecken positioniert sind. Über Infrarotlichtblitze werden reflektierende Kugeln auf Objekten in der CAVE sichtbar gemacht, und mit einem Bildverarbeitungssystem werden die beispielsweise auf der Brille des Betrachters 


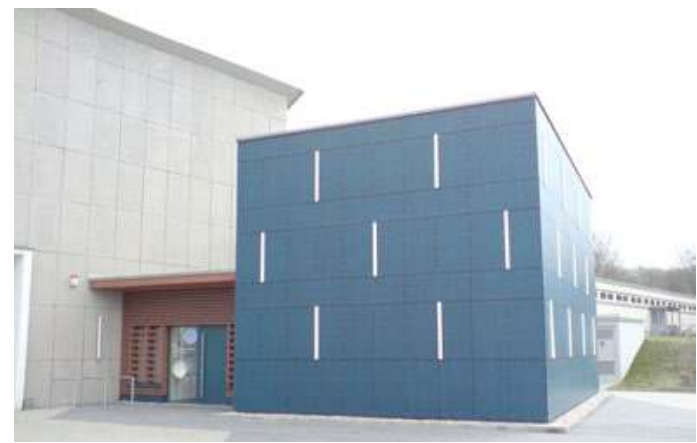

Abbildung 2: Die Außenansicht der CAVE der TU Dresden.

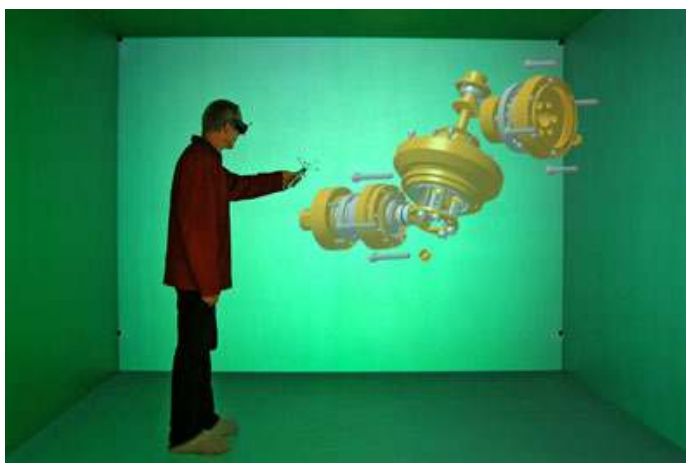

Abbildung 3: Innenraum der CAVE

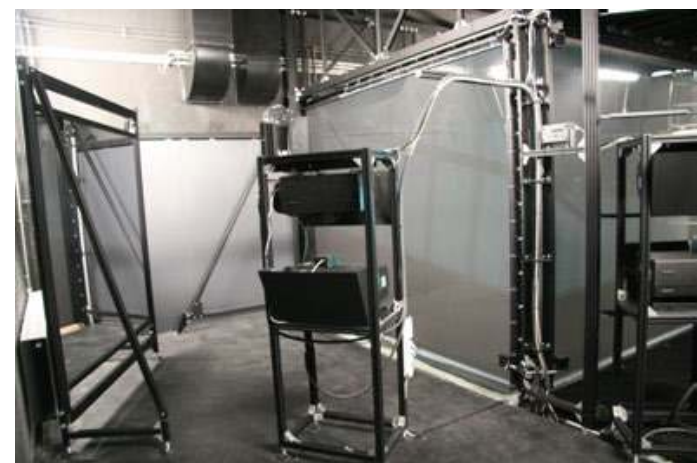

Abbildung 4: Aluminium-Tragkonstruktion der CAVE 
befindlichen Kugelanordnungen (Tracking-Ziele) identifiziert und daraus die Positionsdaten des Betrachters ermittelt (Abb. 5).

Im Rahmen der Produktentwicklung kann eine CAVE für die Bewertung von Produktmodellen in virtuellen Umgebungen eingesetzt werden. Die realitätsnahe Darstellung erweist sich besonders bei komplexen räumlichen Modellen als sehr hilfreich. Bei der Überprüfung der Passfähigkeit von Baugruppen unterschiedlicher Bearbeiter ist das CAD-neutrale Datenformat der visualisierten Modelle von Vorteil.

Besonders kommen diese Effekte in der Fahrzeugentwicklung zum Tragen, wo die Verwendung von Modellen in Originalgröße möglich ist. In deren Innenraum kann beispielsweise die Erreichbarkeit von Bedienelementen und das Sichtfeld untersucht werden. Ein Beispiel für die Zusammenarbeit mit der ansässigen mittelständischen Industrie, in diesem Fall mit der Firma Melkus zeigen die Abb. 6 und 7. Die Geometrie eines historischen Rennwagens wurde im ersten Beispiel per 3D-Scanning rekonstruiert und anschließend unter Verwendung von Menschmodellen (Abb. 7) in der CAVE beurteilt.

Bisher sind moderne Visualisierungstechniken in die tägliche Arbeit eines Produktentwicklers kaum integriert. Ein weiterer Forschungsaspekt ist daher die Einbindung von Anlagen wie der CAVE, in die tägliche Arbeit des Ingenieurs. Hier steht vor allem die einfache Nutzung und Steuerung der virtuellen Umgebung im Mittelpunkt. Der Ingenieur erhält die Möglichkeit, direkt aus dem CAD-System Modelle in der CAVE zu visualisieren oder Bewegungssimulationen in der virtuellen Umgebung zu starten.

Mit der Unterstützung von Professor Dr.-Ing. habil. Ralph Stelzer, Inhaber des Lehrstuhls für Konstruktionstechnik/CAD, stand die CAVE nun auch für das künstlerische Projekt MySpace zur Verfügung.

\section{$5 \quad$ Die Wahl des Titels}

Der Titel der Installation ist eine bewusste Referenz an die Internetplattform mit selbigen Namen (www.myspace.com). Tatsächlich bestehen Ähnlichkeiten im Aufbau der Internetplattform und der Installation in der CAVE. Zunächst gibt es jeweils einen begrenzten Raum, der innerhalb der Regeln frei bespielt werden kann. Es gibt eine vorgegebene Plattform (hier die Internetseite als Plattform, da Kugeln, die mit verschiedenen Inhalten bestückt werden). Sowohl die Internetseite als auch die Kugeln in der CAVE sind virtuelle Räume, in denen virtuelle Realitäten definiert und geschaffen werden können.

Gemeinsam ist auch, dass man, bevor man sich die Internetseite oder die Kugeln anschaut, nicht weiß, was einen erwartet. In virtuellen Räumen sind die Datenmenge, und damit einhergehend die präsentierten Inhalte, mit denen man konfrontiert wird, nicht überschaubar. 


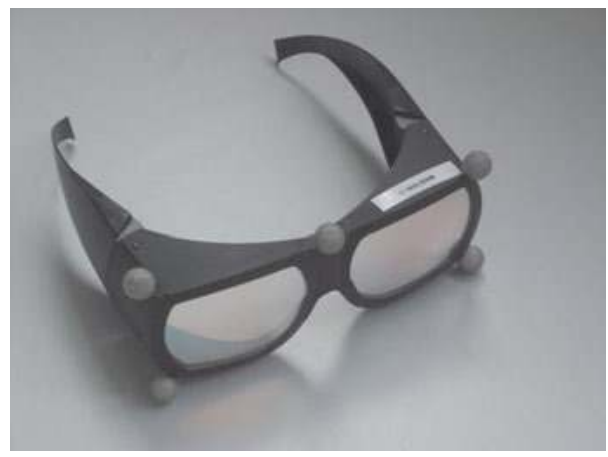

Abbildung 5: Infitec-Filterbrille mit reflektierenden Kugeln als Trackingziele

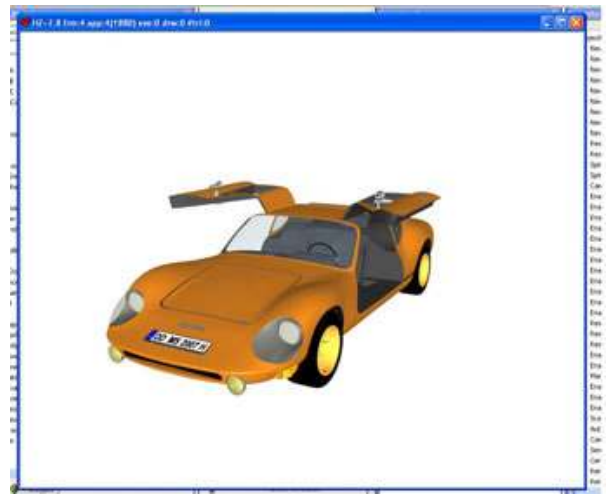

Abbildung 6: Modell des RS 1000

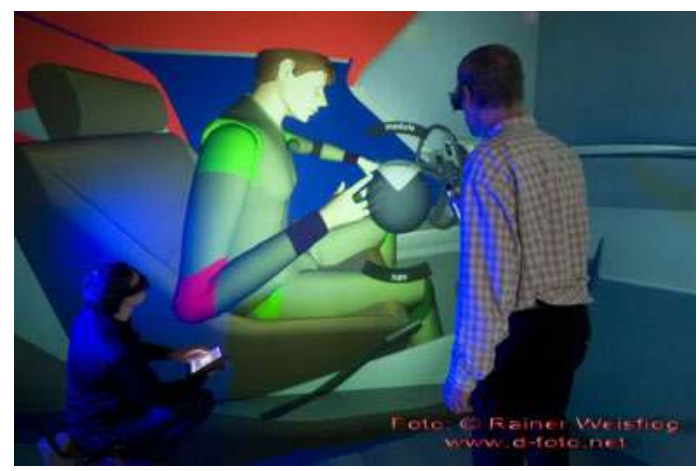

Abbildung 7: Menschmodell 


\section{Das künstlerische Projekt}

Mit dem Projekt MySpace wird eine begehbare virtuelle Welt innerhalb der CAVE der TU Dresden entwickelt, die durch ihr offen angelegtes Gestaltungsprinzip innerhalb eines vorgegebenen Konstruktes eine Vielzahl von möglichen virtuellen Realitäten wiedergibt. Hierbei ist die permanente und fortlaufende Vervollständigung der dargestellten virtuellen Inhalte wünschenswert. Die Studentinnen und Studenten des 3. Semesters (2007) Medieninformatik der HTW Dresden erhielten die Gelegenheit, im Rahmen der zu bestreitenden Projektarbeiten innerhalb ihres Studiums Bestandteile eines aus Kugeln bestehenden Konstruktes mit frei gewählten dreidimensionalen Inhalten zu füllen. Dabei werden Ihnen von der Seite des Künstlers keinerlei inhaltliche Vorgaben oder Beschränkungen gegeben, außer der Einschränkung, dass die Inhalte räumlich in eine Kugel passen müssen natürlich. Die Studierenden konnten Ihrer Phantasie freien Lauf lassen, und es sind sehr unterschiedliche Objekte zu verschiedensten Themen entstanden (siehe Abb. 8). Im Anschluss an die Fertigstellung der Objekte wurden diese zu einem Gesamtmodell in der CAVE zusammengeführt und mit auf die jeweiligen Szenen angepasster Hintergrundsmusik erweitert. Das Konzept der Kugel wurde auch in einigen weiteren Projekten der Konferenz weiterverfolgt. So verwendeten Cornelius Weidner und Rolf Kruse in Ihrer VR-Applikation „Empty Museum - full of Experiences“, die Kugel als Auswahlobjekte zur Navigation und für die Bereitstellung eines Panoramas. Der Benutzer musste in dieser Anwendung ebenfalls in die bereitgestellten Kugeln eintauchen, um entweder eine Auswahl zu treffen oder ein Panorama im Inneren der Kugel zu betrachten. Weit ausgefallenere Interaktionskonzepte und unkonventionelle Interaktionskonzepte, die durchaus eine weitere Betrachtung auch in diesem Rahmen verdienen wurden von Steffi Beckhaus von der Universität Hamburg vorgestellt.

Bei der Interaktion mit der Szene und der Navigation in der CAVE ist entscheidend, dass man mit Hilfe der über die Kameras verfolgten 3D-Brille (Abb. 5) die Möglichkeit bekommt, die Kugeln zu durchdringen und virtuell in deren Körperinneres zu schauen. Dann offenbaren sich dem Betrachter ganz unterschiedliche Inhalte: beispielsweise eine Insel, die sich aus dem Meer erhebt, eine Wüstenwelt oder die Darstellung eines 3D-Tetris-Spiels. Das heißt, der virtuelle Raum ermöglicht, materielle Grenzen von Körpern zu überwinden, Gesetzmäßigkeiten wie z.B. die Schwerkraft auszuschalten, neue Räume mit neuen Realitäten zu entwickeln, kurz: neue Welten zu definieren, zu schaffen. Diese Komponenten machen eine Auseinandersetzung mit virtuellen Realitäten so spannend - eben weil gängige Parameter überwunden und neu definiert werden können. 

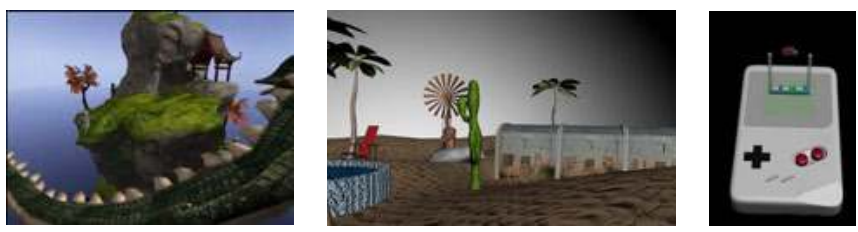

Abbildung 8: Einzelansichten zu verschiedenen studentischen Modellen der Kugelwelten.

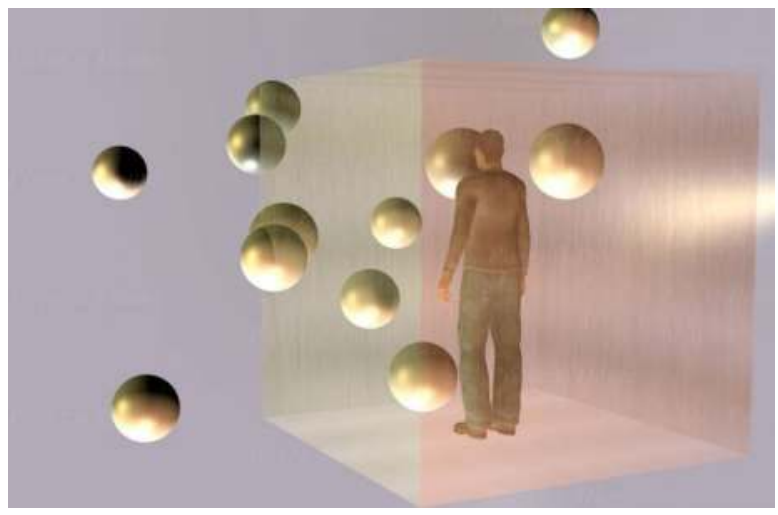

Abbildung 9: Konzept des Betrachters in der CAVE.

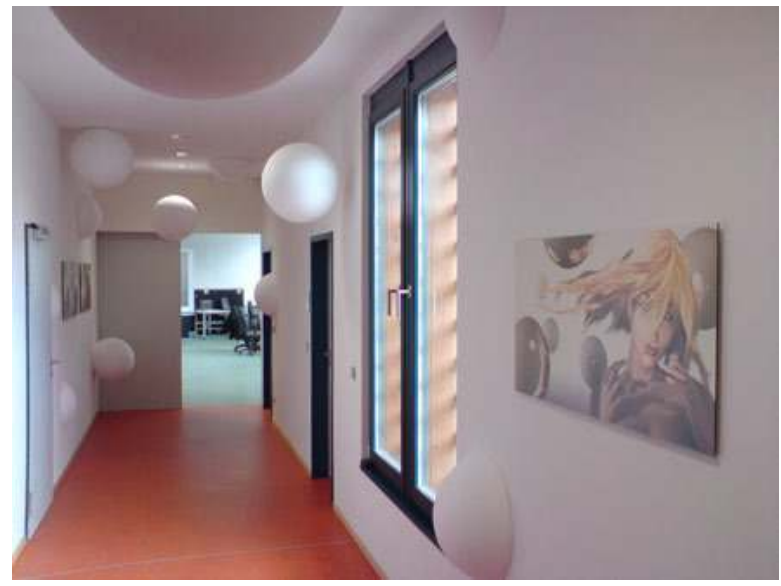

Abbildung 10: Blick in den Flur der CAVE mit der Begleitausstellung von Styroporkugeln und Digitaldrucken. 
Ein Besuch der Ausstellung und eine Besichtigung der virtuellen Welt in der CAVE gestalten sich wie folgt: Es gibt ein Konstrukt, bestehend aus einer Vielzahl von Kugeln, die innerhalb der CAVE eine Erscheinungsgröße von ca. einem Meter haben. Alle Kugeln „schweben“ im Raum und bewegen sich auf definierten und wiederholenden Bahnen, bzw. der Raum bewegt sich mit seinem gesamten ,Kugelinhalt“.

Jeder beteiligte Student hat eine Kugel mit einem bestimmten Inhalt seiner Wahl bestückt, bestehend aus einer modellierten 3D-Szene mit entsprechender Textur, Beleuchtung und gegebenenfalls Animation.

Die in der CAVE dargestellten Kugeln erscheinen von außen betrachtet als Körper mit geschlossener, weißer Oberfläche und Textur (Abb. 9).

„Durchbricht“ der Betrachter mit dem Kopf die Kugeloberfläche, so wird für ihn auch ihr Inneres sichtbar. Dieser Kugelinhalt wurde von den Studenten der HTW Dresden modelliert und gestaltet. Dabei sind die beteiligten Studenten für den Inhalt ihrer Kugeln selbst verantwortlich und erhielten vom Künstler lediglich Anregungen oder Richtungen, in die es bei der Bestückung der Kugeln gehen könnte (siehe Abb. 8). Die Interaktion des Betrachters beschränkt sich nicht nur darauf, dass er in die ankommenden Kugeln hineingehen und sie wieder verlassen kann, er steuert auch deren Geschwindigkeit und den an die Kugelwelt gekoppelten Sound. Die Kugel wird beim Eindringen um ein vielfaches langsamer auf ihrer Bahn und gewinnt wieder an Geschwindigkeit, sobald der Betrachter die Kugel verlässt. Er kann also selbst beeinflussen wie lange er sich den jeweiligen Kugelinhalt anschaut. Zusätzlich zu den präsentierten Kugelinhalten wurde eine musikalische Atmosphäre definiert, die die räumliche Wahrnehmung des Betrachters um ein Vielfaches erweitert. Der Sound einer jeden Kugel wird von der Seite langsam eingeblendet, von der sie sich einem Betrachter nähert und steigert sich noch einmal sprunghaft, wenn dieser die Kugeloberfläche „durchbricht“. Hier wird der Sound der sonoren Hintergrundatmosphäre unterbrochen. Die durch die moderne Anlage der CAVE wiedergegebene klangliche dreidimensionale Atmosphäre erweitert dabei die räumliche Wahrnehmung des Betrachters um ein Vielfaches.

\section{$7 \quad$ Begleitausstellung}

Parallel zu der öffentlichen Präsentation der CAVE-Installation „MySpace“ präsentiert Matthias Lehmann eine Begleitausstellung, die die Anlage des virtuellen Raumes in den materiellen Raum, nämlich den Eingangsbereich der CAVE überträgt. Zum einen ist eine Installation aus Styroporkugeln (Abb. 10) zu sehen, welche das Prinzip der CAVE insofern aufgreifen, als dass eine Welt innerhalb eines vorgegebenen Rahmens erschaffen wird: Eine Welt in einer Welt in einer Welt. Wesentlicher Unterschied ist dabei zum einen, dass in materiellen Räu- 
men die Begrenztheit der Welt in der Welt wesentlich schneller ersichtlich und darüber hinaus haptisch begreifbar ist. Zum anderen suggerieren die Styroporkugeln im Eingangsbereich dem Betrachter, dass diese alle eine Welt in der Welt besitzen. Nur überprüfen kann man diese Behauptung nicht, da man im Falle der Styroporkugeln sehr schnell mit ihren materiellen Grenzen konfrontiert ist. Im virtuellen Raum hingegen bleibt der Kugelinhalt virtuell aber bei jeder Kugel überprüf- und erlebbar, haptisch erfährt man im ungünstigsten Fall lediglich die schmerzliche Materialität der Projektionsflächen oder der Tür der CAVE. Zusätzlich präsentiert Matthias Lehmann Assoziationen zu VR anhand von vier Digitaldrucken auf Alu-Dibond (Abb. 10).

\section{Veranstaltungen}

Die Vernissage zur Ausstellung fand am 14. März 2007 an der CAVE mit großer Publikumsresonanz statt. Die Studentinnen und Studenten haben in dem Projekt enorme Ausdauer und Engagement gezeigt und außerordentliche Modellierergebnisse erzielt. Zu den Ergebnissen ist zu ergänzen, dass das Projekt für den größten Teil der Studierenden der erste Kontakt mit einem großen Modellierprogramm wie 3D Studio Max und das erste Projekt im Bereich der Computergraphik war. Der Erfolg der Studenten spiegelt sich zudem in Praktikumsangeboten verschiedener Firmen wieder, deren Vertreter bei der Eröffnung anwesend waren. Ganz besonderen Dank für den unermüdlichen Einsatz in der CAVE geht an dieser Stelle an Tino Grimmer, Michael Wegner und Erik Steindecker. Die Besichtigung der Ausstellung ist auf Anfrage an die Mailadresse myspace@mailbox.tu-dresden.de nach Absprache von Terminen prinzipiell jederzeit möglich.

\section{Literatur}

Frieling, R./Danields, D.: Medien Kunst Interaktion, 2000.

Jokisch, R.: Virtualität oder Cyberspace, 2000 〈URL: http://www.tu-berlin.de/ $\sim$ society/Jokisch_GB_Virtueller_Raum.htm $\rangle$.

Wikipedia: Virtuelle Realität, 〈URL: http://de.wikipedia.org/wiki/Virtuelle_ Realität - Zugriff am 24.03.2008.

Zweck, A.; Zukünftige Technologien Consulting (Hrsg.): Virtuelle Realität Spiel oder Kultur prägender Faktor? Düsseldorf 2006, S. 9. 



\section{Tim Jansen/Roland Kuck}

\section{Interaktion in virtuellen Welten}

Eine kollaborative Anwendung für virtuelle Umgebungen zu schaffen stellt besondere Anforderungen an das 3D Userinterface. Im folgenden beschreiben wir die Anwendung, die im Projekt Virtualisierung von Skulptur ${ }^{1}$ enstanden ist. Es galt kunsthistorischen und medientheoretischen Wissenschaftlern zu ermöglichen, ernsthafte Analysen durchzuführen. Das Interface ermöglicht unter anderem eine intuitive Navigation durch die virtuelle Galleria Borghese, sowie die Manipulation von virtuellen Skulpturen. Die entwickelte AVANGOAnwendung $^{2}$ in Kombination mit 3D Userinterface-Techniken macht diese komplexe Interaktion mit der virtuellen Welt möglich

\section{$1 \quad$ Kontext}

Um ein Museum als komplexes Gebilde sinnlicher Wahrnehmung in einer virtuellen Realität ernsthaft erfahrbar werden zu lassen, bedarf es erheblicher Grundlagenforschung und ausgefeilter Technik. Der Grad dieses Aufwands hängt dabei von der Zielgruppe ab, für die es bestimmt ist. Ein unvoreingenommener Museumsbesucher verfolgt andere Ziele und besitzt andere Voraussetzungen als ein ausgebildeter Kunsthistoriker. Während der normale Museumsbesucher eher die Wahrnehmung des Museums im Ganzen beabsichtigt ohne wirklich einzelne Aspekte bestimmter Exponate im Detail zu beleuchten, zielt die Wissenschaft meist auf tiefgehende Untersuchungen spezieller Objekte. Um der Detailtiefe gerecht zu werden, die ein Kunsthistoriker zur Analyse benötigt, müssen alle Komponenten des Systems akribisch aufeinander abgestimmt sein.

Die Grundlage der Untersuchungen bildet die barocke Skulpturgruppe Gian Lorenzo Berninis (1598-1680) in der Villa Borghese in Rom. Die überlebensgroßen Figuren in ihrer filigranen Ausarbeitung und ihrem kompakten Erscheinungsbild eignen sich hervorragend für die verschiedenen Fragestellungen. Zudem wirft die Skulpturengruppe Berninis eine konkrete Diskussionsfrage auf. Es ist zu untersuchen wie das Arrangement der Skulpturen in der Villa damals von Bernini geplant war. Die Villa Borghese wurde in den letzten Jahrhunderten mehrfach umgestaltet. Nur aus verschiedenen literarischen Ausführungen gibt es konkrete Vorschläge dafür, wie die Gestaltung der Villa einst ausgesehen haben könnte. In einem virtuellen Abbild der Villa inklusive der Skulpturgruppe könnten Wissenschaftler mit einfachen Mitteln die Aufstellung und das Zusam-

1 Gefördert im Forschungskolleg 615 Medienumbrüche der Deutschen Forschungsgemeinschaft (DFG).

2 Für AVANGO siehe Kuck et al.: Improving the AVANGO VR/AR Framework. 


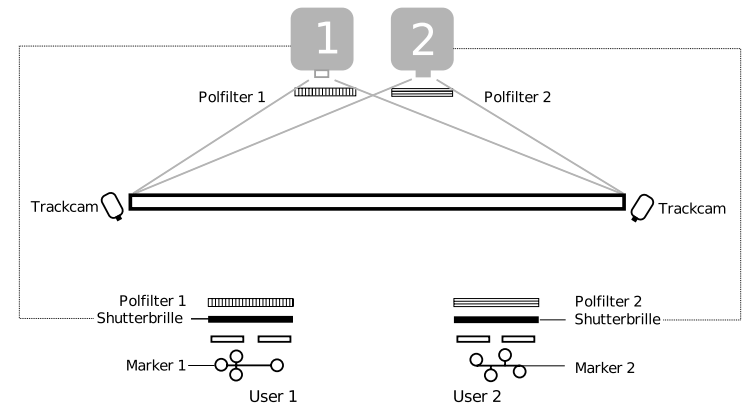

Abbildung 1: Aufsicht der TwoView-Displaykomponenten

menspiel der Skulpturen erproben und so wichtige Anhaltspunkte zur originalen Aufstellung erarbeiten.

Um eine angemessene und zielgerichtete Interaktion zu gewährleisten, ist das Interaktionsinterface und sein Funktionsumfang auf die speziellen Bedürfnisse der Zielgruppe und der Aufgabenstellung anzupassen. Diese Zielgruppe besteht in erster Linie aus Wissenschaftlern, die aus dem medientheoretischen und kunsthistorischen Bereich kommen. Durch diesen eingegrenzten Anwenderkreis ist es möglich, den Funktionsumfang stark auf die Bedürfnisse der Zielgruppe anzupassen und somit gewisse Vorraussetzungen für die Nutzung des Interfaces einzuführen. Es wurden ein Werkzeug zur Analyse kunsthistorisch relevanter Aspekte in Gebäuden, wie z.B. Museen, entwickelt. Da die Zielgruppe wenig Erfahrung in Computertechnik sowie der Nutzung von Interfaces in Stereoprojektionen hat, wurde auf intuitive Arbeitsabläufe und eine schnell erfassbare Interfacenavigation geachtet.

\subsection{TwoView-Display-System}

Um eine kollaborative Nutzung einer virtuellen Umgebung gewährleisten zu können, wurde am Fraunhofer IAIS das Multi-Viewer-Display TwoView ${ }^{3}$ entwickelt, welches es ermöglicht, zwei Anwendern zeitgleich ein separates stereoskopisches Bild zu bieten. Somit wird erreicht, dass beide Anwender ein räumlich absolut korrektes Bild wahrnehmen. Dies unterstützt die Präsens und die Echtheit der virtuellen Umgebung in hohem Maße und bietet erstmals die Grundlage für die ernsthafte kollaborative Analyse komplexer virtueller Daten. Das Projektionsverfahren besteht aus einer Kombination aus aktivem und passivem Stereobild, wie optischem Tracking zur Bestimmung von Betrachtersichtwinkel und -position. Der Aufbau des Systems ist in Abb. $1 \mathrm{zu}$ sehen. Das grafische System

3 Fraunhofer IAIS: TwoView/L-Shape. 
berechnet mit Wissen über die genauen Translations- und Orientierungskoordinaten der beiden Anwender separate Perspektiven der virtuellen Umgebung. Diese beiden Perspektiven werden jeweils über einen Stereoprojektor auf eine Projektionsfläche gesendet. Die Trennung der beiden Perspektiven wird über die unterschiedliche Polarisation der beiden Projektorbilder erreicht. Da diese Perspektiven aus stereoskopischen 3D-Bildern bestehen, werden diese über aktive Shuttertechnik separiert und den entsprechenden Augen der Betrachter zugewiesen.

\section{Interface}

Um einen Überblick über den Arbeitsablauf und die Gestaltung der Funktionselemente zu bieten, wird im folgenden eine detaillierte Beschreibung der Arbeitsschritte einer möglichen Nutzung des Interfaces aufgeführt. Dieser Ablauf behandelt nicht alle Features des Interfaces, sondern nur die Hauptfunktionen mit dem idealen Arbeitsablauf.

Der Anwender kontrolliert die Anwendung über einen PDA, der drahtlos mit dem Anwendungsrechner verbunden ist. Der erste Schritt einer jeden Nutzung ist daher die Verbindung zum AVANGO-Server. Nach erfolgreicher Verbindung steht das Hauptmenü zur Auswahl. Um einen Überblick über die Positionen der Skulpturen und des eigenen Standpunktes sowie eine Orientierung in der Umgebung zu schaffen, wird der Menüpunkt position angewählt. Hier erscheint der Grundriss der Galerie sowie die Positionen aller Objekte. Nun kann der Anwender ein vorgefertigtes Setting anwählen (siehe Abb. 2), um sich über bisherige Vorschläge der Positionierung zu informieren. Hat er sich für ein Positionssetting entschieden, kehrt er zum Hauptmenü zurück.

Um sich nun das Setting im Kontext der Galerie und der anderen Skulpturen anzuschauen, kann der Anwender in früheren Begehungen für gut befundene Touren, speziell für dieses Setting, anwählen. Wichtig ist, dass es für jedes vorgefertigte Positionssetting, speziell für dieses, individuell gefertigte Touren zur Auswahl stehen. Der Anwender wählt im Hauptmenü den Menüpunkt tourguide. Um eine effiziente Suche zu gewährleisten, wird durch die Wahl einer Kategorie die Auswahl eingeschränkt.

Die Kategorien sind:

- Skulpturen: Unter diesem Punkt werden Rundtouren um jede Skulptur, die sich in der Galerie befindet, angeboten.

- Räume: Es werden Touren angeboten, die die Skulptur in Beziehung zu dem Raum, in dem sie sich befindet, thematisiert. Es spielt nicht nur die Skulptur die vorherrschende Rolle, sondern in erster Linie die Raumbeschaffenheit. 
- Gebände: In dieser Kategorie können Touren durch das ganze Gebäude gefunden werden. Sowohl zwischen zwei Räumen als auch durch die ganze Galerie.

Ist die Kategorie gewählt, erhält der Anwender die möglichen Touren in einer Liste (siehe Abb. 2). Auf Grund der Tourvorschau im Grundriss trifft er seine Wahl und startet die Begehung.

Während der Begehung sorgt das Play-Panel für die Kontrolle über die verschiedenen Parameter der Navigation (siehe Abb. 3). Es lässt dem Anwender genug Raum, um voll und ganz in die virtuelle Welt einzutauchen, ohne sich um schwierige Navigationsaufgaben sorgen zu müssen. Durch Änderung der Position des mobilen Gerätes auf der horizontalen Ebene kann der Anwender die Geschwindigkeit beeinflussen. Die relative Translation wird zur Erhöhung und zur Verringerung der Geschwindigkeit genutzt. Sind die Werte zur vorhergehenden Position niedriger, d.h. hat der Anwender das Gerät heran gezogen, verringert sich die Geschwindigkeit der Fahrt. Schiebt der Anwender das Gerät jedoch von sich weg, wird die Fahrt je nach Abstand beschleunigt. Ein Schwellwert filtert geringfügige und ungewollte Veränderungen der Position heraus. Ein Button, der im walkaround-Modus aktiv ist und eine gewisse Größe der Oberfläche beansprucht (wenig Ablenkung des Anwenders durch Steuerung), ist für Play/Stop zuständig.

Um die Position einer Skulptur zu modifizieren, wird erneut der Hauptmenüpunkt position angewählt. Über die Wahl modify set erscheint der Screen zur Positions- sowie Rotationsmanipulation (siehe Abb. 4). In diesem kann nun die gewünschte Manipulation der Skulpturen sowie des Viewers vollzogen werden. Optional kann die Zoomfunktion zur akkuraten Ausrichtung herangezogen werden. Sind alle Ausrichtungen durchgeführt, kehrt der Anwender zum Hauptmenü zurück.

Um sich nun das modifizierte Positionssetting in einer Begehung anzuschauen, wird der Hauptmenüpunkt Pfaderstellung gewählt. Über den ersten Screen dieses Funktionszweigs können zur Laufzeit erstellte Pfade erneut begangen, sowie zur Editierung angewählt werden. Ebenso kann die Erstellung eines neuen Pfades initiiert werden. Es erscheint der Grundriss zur Pfaderstellung. (siehe Abb. 3)

Hier legt der Anwender zuerst die Start- und Endpunkte des Pfades durch einfache Klicken auf den Grundriss fest. Danach wird ein dazu passender Pfad berechnet und auf dem Grundriss eingezeichnet. Der Anwender hat jetzt die Möglichkeit weitere Punkt auf dem Grundriss zu markieren. Der Pfad wird dann so angepasst, dass er durch alle so markierten Punkte läuft.

Ist ein Pfad erstellt, so gelangt der Anwender in den Play-Modus, der die gleichen Funktionen enthält, wie zuvor in der Begehung einer Tour beschrieben. 

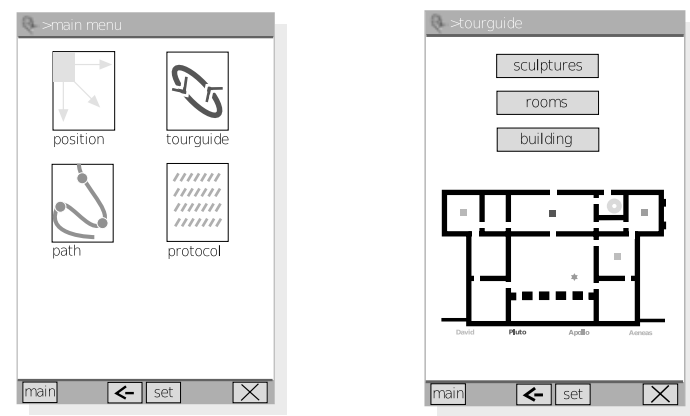

Abbildung 2: links: Hauptmenü, rechts: Auswahl einer Tour
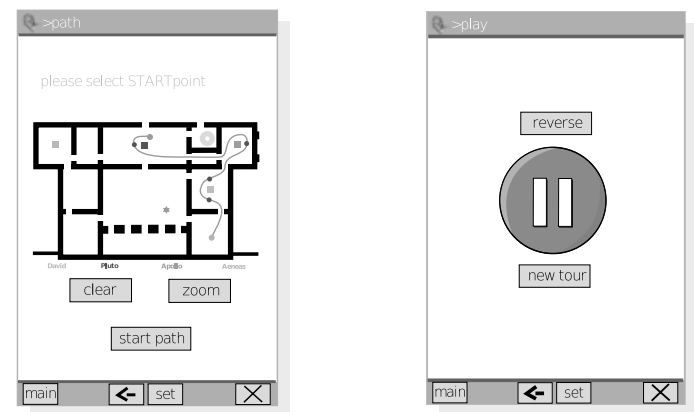

Abbildung 3: links: Abspielmodus, rechts: Bearbeiten eines Pfades
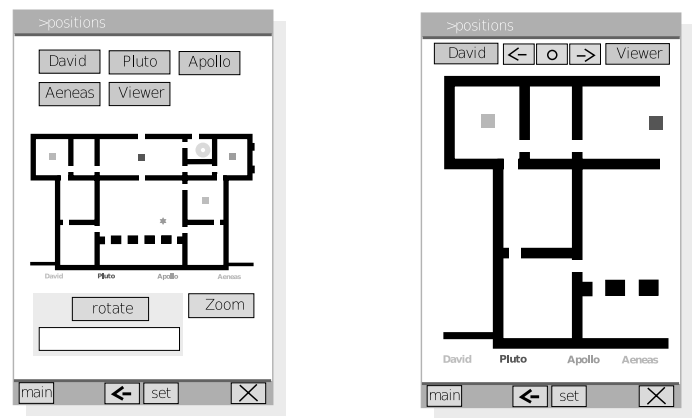

Abbildung 4: links: Positionierung der Skulpturen, rechts: Zoom-Ansicht 
Die Anwendung kann jederzeit über die Exit-Funktion beendet werden und ebenso ein erneuter Verbindungsaufbau angestoßen werden.

\section{Pfadberechnung}

Da die Pfadberechnung eine wichtigen Einfluss auf das Erleben der virtuellen Welt hat, wird diese im folgenden ausführlich beschrieben.

Das Pathfinding spielt als Teilbereich der Künstlichen Intelligenz in Computerspielen schon seit vielen Jahren eine große Rolle. Sowohl im 2D als auch im 3D Bereich ist so der Einsatz von intelligent wirkenden Computergegnern erst möglich. Aber auch als Navigationsform in 3D Umgebungen wird das Pathfinding neben der kontinuierlichen Eingabe von Navigationsbefehlen zunehmend eingesetzt. Auch in Anwendungen jenseits des Spielgenres findet diese Methode Verwendung, wie z.B. in Routenplanern und Navigationssystemen. Generell gehört das Pathfindung in das Gebiet der Terrain Analysis. Ziel ist die Generierung eines möglichen Weges zwischen zwei Punkten in einem gegebenen geografischen Suchraum. Der gesuchte Weg kann verschiedene Anforderungen besitzen. Es kann der kürzeste Weg zum Ziel, es kann irgendein Weg zum Ziel oder auch der Weg über einen dritten Punkt zum Ziel gesucht werden. Der grundlegende Faktor ist der Suchraum, der mit seinen vielseitigen Charakteristiken die Komplexität des Algorithmus stark beeinflusst.

\subsection{Partitioning}

Der erste Schritt ist die Vorbereitung des Suchraumes (visuelle Repräsentation), um im folgenden die Analyse zur Berechnung des idealen Pfades durchführen zu können. Diese Überführung der geografischen Karte in eine logische Repräsentation wird auch als Partitionierung bezeichnet. Die logische Repräsentation besteht meist aus einem ungerichteten Graphen mit einer unbestimmten Anzahl an Knoten und Kanten. ${ }^{4}$ Die Knoten repräsentieren einen bestimmten Teilbereich der Umgebung. Knoten, dessen Teilbereiche in der Umgebung nebeneinander liegen, werden durch Kanten miteinander verbunden. Diese Kanten haben jedoch keine vorgegebene Laufrichtung, sondern sind in beide Richtungen kombinierbar, d.h. von Knoten $A$ ist Knoten $B$ über Kante $k$ erreichbar und von $B$ ist $A$ über $k$ erreichbar. Es gibt verschiedene Verfahren das visuelle Modell in die logische Repräsentation umzuwandeln, z.B. durch Wegpunkte-Graphen oder durch Polygonale Zerlegung. Die Wahl der Technik hängt stark von der Beschaffenheit des Suchraumes ab. Liegt ein statischer Suchraum vor, kann das visuelle Modell manuell vorbereitet werden und es entfällt der hohe Performanceaufwand zur Berechnung der Partitionierung. Sind

4 Kastenholz: 3D-Pathfinding. 
jedoch dynamische Elemente Bestandteil der visuellen Repräsentation, muss die logische Repräsentation dynamisch generiert werden.

Im Fall der Anwendung dieses Projektes lag es nahe, die logische Repräsentation der virtuellen Umgebung über ein gleichmäßiges Raster zu erstellen. Da Betrachterhöhe bzw. Terrainhöhe gleichbleibend sind, konnte die dritte Dimension vernachlässigt werden. Weil jedoch dynamische Objekte in Form von Skulpturen vorhanden sind, konnte das Raster nicht ausschließlich manuell bereitgestellt werden, sondern muss zum Teil auf Grundlage des manuellen Rasters generiert werden. Im Gegensatz zu der üblichen Vorgehensweise, die Umgebung in kleine Parzellen zu unterteilen, wurde das Gebäude auf Grund der rechteckigen Raumform in ein großes Raster unterteilt. Jeder Raum repräsentiert einen Knoten in der logischen Repräsentation. Dementsprechend stellt jede Tür eines Raumes (Knotens) die Kante zu dem Nachbarraum(-knoten) dar.

So entstand die manuell vorgefertigte logische Repräsentation, die die statischen Teile der visuellen Repräsentation beinhaltet. Um den Graphen zu vervollständigen, müssen nun auf Grund der Skulpturpositionen die Subräume generiert werden. Enthält einer der Räume ein dynamisches Objekt (Skulptur), so wird er in vier Subräume unterteilt, die das Objekt umschließen (siehe Abb. 5). Diese Subräume sind gleichzusetzen mit Subknoten und ersetzen den zugehörigen Vaterknoten. Die Subräume, die geografisch im Bereich einer Tür des Vaterraums liegen, übernehmen diese Tür. Um von Subraum zu Subraum zu gelangen ist es nötig auch hier imaginäre Türen zu schaffen. Die jeweiligen Nachbarknoten erhalten relativ zum Objekt (Skulptur) die zugehörige Kante. Ist diese Generierung der Subgraphen durch alle Objekte erfolgt, ist die logische Repräsentation vollständig.

Auf Grund dieses Graphen (logischen Repräsentation) ist nun ein Algorithmus anzuwenden, der den kürzesten Weg innerhalb dieses Graphen findet. Eingangsparameter für graphenbasierte Algorithmen sind der Startknoten, der Endknoten und der Graph selbst. Es gibt verschiedene Algorithmen, die für diese Aufgabe geeignet sind, wie z.B. der Dijkstra- und der verwandte AstarAlgorithmus ${ }^{5}$. Diese Algorithmen arbeiten mit Gewichtungen der Knoten und der Kanten des Graphen auf der Suche nach dem Zielknoten. Im Bernini-Projekt wurde die Astar-Methode verwendet, die nicht nur die einfache Gewichtung aller Knoten berücksichtigt, wie die Dijkstra-Methode, sondern über eine HeuristikFunktion Schätzungen über die Restdistanz zum Zielknoten vornimmt.

\subsection{Berechnung der Betrachtersicht}

Auch die Blickrichtung muss vom System berechnet werden. Es existieren zwei Modi: Zum einen eine Betrachtersicht die tangential zum Kamerapfad folgt.

5 Lester: A* Pathfinding. 

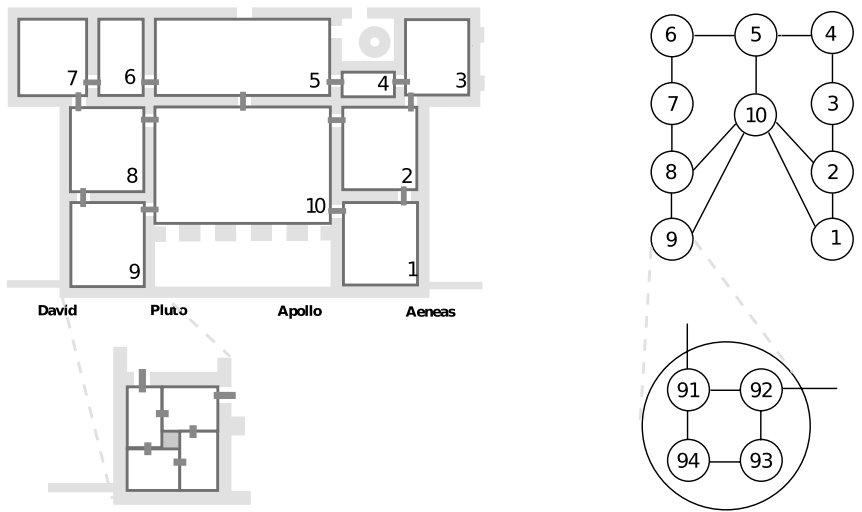

Abbildung 5: Raumsegmentierung und zugehöriger Graph

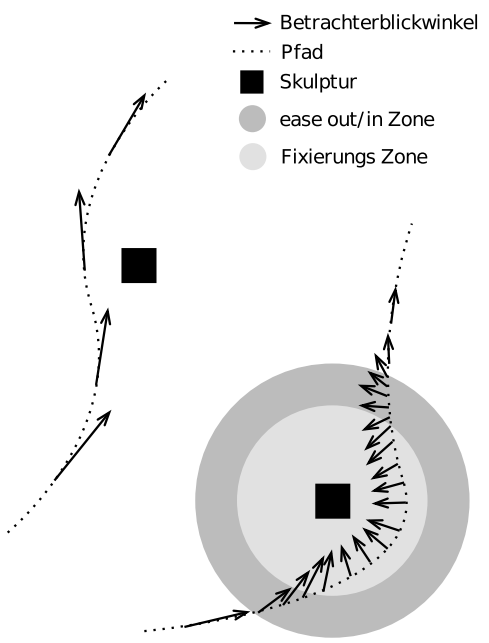

Abbildung 6: Steuerung der Blickrichtung 
Zum anderen eine Betrachtersicht, die interessante Objekte anvisiert, sobald eine bestimmte Reichweite zum Objekt erreicht wird. Interessante Objekte sind im Fall der Bernini-Anwendung alle Skulpturen, die sich in der Galerie befinden. Sobald sich ein Punkt auf dem berechneten Pfad in die Gravitationsreichweite eines Objektes bewegt, wird der Blickwinkel auf die Skulptur, abhängig von dem Pfadpunkt und der Skulpturposition, neu berechnet. Um eine weiche Interpolation zwischen dem normalen tangentialen Blickwinkel und dem Blickwinkel zum Objekt zu errechnen, wird mit Hilfe einer Gewichtung ein weicher Übergang geschaffen. Dies wird in Abb. 6 gezeigt.

\subsection{Mehrere Wegpunkte}

Die vom Anwender gesetzten Wegpunkt zwischen Start- und Endpunkt werden nicht in der Reihenfolge des Setzens abgelaufen. Stattdessen wird für alle möglichen Permutationen der Pfad nach der oben beschriebenen Methode berechnet. Aus all diesen Pfaden wird dann derjenige ausgesucht, der die kleinste Weglänge hat.

\section{Evaluation}

Zur Evaluation des Userinterfaces wurden mehrere Aufgaben definiert und anschliessend analysiert.

\subsection{Aufgaben}

Um in der Evaluation vergleichbare Ergebnisse zu erzielen, wurden Aufgabenstellungen erarbeitet. Im folgenden werden die Aufgabenstellungen der einzelnen Usertest kurz beschrieben.

\section{Usability-Test Nr. 1}

Der erste Usertest konzentrierte sich auf die Auswahl von geführten Touren, dem Tourguide. Da dies der erste Test war, wurde ebenso die Menüführung des 2D Interfaces auf die Probe gestellt. Zu Anfang des Tests wurde eine kurze Aufwärmphase in der Nutzung des Interfaces durchgeführt, um dem Anwender das Gefühl der Sicherheit zu geben und um die Prüfungssituation zu entspannen. Folgend bestand die Aufgabe darin, eine ganz bestimmte Tour durch die virtuelle Villa im Tourguide-Menü auszuwählen und diese mit Hilfe der Geschwindigkeitssteuerung an einer vorgegebenen Position zu stoppen. Die zweite Aufgabe beschäftigte sich ebenfalls mit der Geschwindigkeitssteuerung während der Begehung der Tour. Folgend wurde die Kategorisierung der Touren und somit die Menüführung gestestet. Mit einer kollaborativen Nutzung wurde der praktische Test beendet. 


\section{Usability-Test Nr. 2}

Im zweiten Usability-Test wurde das umfangreichste Modul getestet. Die Pfaderstellung stellt die höchsten Herausforderungen in ihrer Nutzung an den Anwender und somit auch an die Entwicklung des Interaktionsdesigns. Das Verständnis der Pfaderstellungstechnik wurde eruiert, indem verschiedenen Szenarien zur Aufgabe gestellt wurden. Es wurde z.B. die Planung von Pfaden in einem Raum, durch mehrere Räume, mit verschiedenen Wegpunkten und im Zusammenspiel von Skulpturen getestet. Dies wurde in Kombination mit verschiedenen Positionssettings, die der Proband selbständig je nach Aufgabe wählte, durchgeführt. Durch diese Aufgaben wurde zudem automatisch die Objektkodierung (Skulpturfarbgebung) im 2D-Grundriss und die automatische Fixierung der Skulpturen in der virtuellen Umgebung getestet.

\section{Usability-Test Nr. 3}

Der dritte und für diesen Prototypen abschließende Test behandelte in erster Linie die Positionierung sowie das Zusammenspiel aller implementierter Komponenten der Anwendung. Die Aufgaben bestanden aus zwei Teilen: Zum einen die Manipulation der Positionierung und zum zweiten die Navigation. So wurde z.B. die Kombination aus Positionierung zur Pfaderstellung und Positionierung zum Tourguide getestet. In der Positionierung wurden die verschiedenen Zielpositionen und Techniken, wie z.B. im Zoom-Modus positionieren, vorgegeben. Besonderer Testbereich in diesem Test war die Pfaderstellung in Kombination mit frei positionierbaren Objekten und in Bezug auf die automatische Skulpturfixierung.

\subsection{Auswertung}

Die folgenden Auswertungen werden aus den drei Hauptevaluationsmethoden zusammengefasst und zeigen somit die Tendenzen der Usability-Tests auf. Es wurden jeweils insgesamt sechs Probanden getestet, die sich im Alter zwischen 28 und 64 Jahren befanden. Die Hälfte der Probanden kamen aus dem kunsthistorischen Bereich und waren Neulinge auf dem Gebiet der Interaktion in virtuellen Umgebungen. Die übrigen Probanden waren erfahrene Anwender.

\section{Usability-Test Nr. 1}

In der Kennenlehrnphase wurde bereits klar, Dass die visuelle Gestaltung des Interfaces durchweg positiv aufgenommen wurde. Auch die Menüführung wurde nach kurzem Gebrauch sehr schnell als intuitives Werkzeug angenommen und verstanden. Nur kleine Änderungen der Menüführung ergaben sich aus den Tests, wie die Reihenfolge der Hauptmenüpunkte, die Notwendigkeit einer 
erneuten Bestätigung des Beendens der Anwendung und die Button-Position der Hauptsettings. Im Tourguidemenü wurde die Kategorisierung der Touren als verständlich empfunden, jedoch gab es Schwierigkeiten mit den Bezeichnungen (Namen) der Touren. Diese sollten eindeutiger in ihrer Aussage sein und schon zur Auswahl der Tour beitragen.

Insgesamt wünschten sich die Probanden eine größere Auswahl an vorgefertigten Touren. Die Vorvisualisierung der Tour im 2D-Grundriss wurde als sehr hilfreich empfunden. Während der Begehung wurde die Geschwindigkeitssteuerung als sehr intuitiv und leicht justierbar empfunden. Die Probanden störte jedoch die mangelnde Freiheit in der Navigation. Es wurden verschiedene Möglichkeiten gewünscht, wie das rückwärts Abspielen einer Tour und die Manipulation der Betrachterhöhe. Zudem wurden verschiedene Verbesserungsmöglichkeiten in der Steuerung einer Tour eruiert.

\section{Usability-Test Nr. 2}

Trotz einiger Änderungen, die auf Grund des ersten Usertests vorgenommen wurden, fanden sich die Probanden mit Hilfe der wenigen Erfahrung mit dem Interface aus dem ersten Test sehr gut zurecht. Dies spricht für ein nachhaltiges und gut erlernbares Interaktionsdesign.Während der Aufgaben zur Pfaderstellung wurde klar, dass diese eine gewisse Eingewöhnungsphase benötigt. Je nach computertechnischer Erfahrung des Users wurden hier starke Unterschiede im Verständnis und in der Umsetzung erkannt. Nach dieser Eingewöhnungsphase war es den Anwendern jedoch sehr schnell klar, wie das Interface zu bedienen ist, um gewünschte Pfade zu erhalten.

Die Technik wurde im nachhinein als sehr praktikabel empfunden. Die in Abschnitt 3 vorgestellten Techniken zur Pfaderstellung könnten in weiteren Tests evaluiert werden und auf Erlernbarkeit, Qualität und Schnelligkeit mit der implementierten Technik verglichen werden. Die Probanden wünschten sich jedoch eine Möglichkeit, den Pfad detailreicher erstellen zu können und regten eine Zoomfunktion an. Des weiteren wurde klar, dass um die einwandfreie Nutzung zu gewährleisten, Informationen und Feedbacks während der Pfaderstellung sehr hilfreich sein würden.

Während der Begehung des Pfades wurde die Skulpturfixierung als sehr ruckartig empfunden, d.h. das Umschwenken zur Skulptur und wieder zurück war eine sehr abrupte Bewegung. Der Test deckte bezüglich der Skulpturfixierung einige Mängel auf, die folglich behoben werden konnten. Es wurden von Test zu Test Variationen der Farbkodierung der Skulpturen im 2D-Grundriss implementiert, um ein ideales Ergebnis zu erzielen. Auch im zweiten Test war diese noch nicht angemessen. 


\section{Usability-Test Nr. 3}

Im dritten Test wurde in erster Linie die Positionierung als neues Modul getestet. Um Aufschlüsse über das Zusammenspiel erschließen zu können, wurden jedoch die Positionierung mit den übrigen Funktionsgebieten verknüpft. Die Positionierungstechnik wurde von den Probanden als gut empfunden. Auch der Zoom-Mode trug zu diesen Ergebnissen bei. Die Probanden konnten alle Objekte nach ihren Wünschen platzieren. Durch eine tiefergehende Feinjustierung könnten diese jedoch verbessert werden. Die Rotation von Objekten wurde von allen Probanden als intuitive Technik gewertet. Während der Positionierung waren die erfahrenen Probanden, die an den ersten Tests teilgenommen hatten, klar im Vorteil.

Erstnutzer mussten sich in die Technik einarbeiten. Hier ist auf die Evaluation der verschiedenen Pfaderstellungstechniken zu verweisen, wobei die implementierte Technik im Nachhinein als gut eingestuft wurde. Nur die Fixierung der Skulpturen entsprach noch nicht ganz den Ansprüchen der Anwender. Um eine weiche Interpolation in allen Kombinationen von Skulpturaufstellungen gewährleisten zu können, muss dieses Verfahren verfeinert werden. Die erfahrenen User wünschten sich als ergänzende Navigationsmethode eine freie Navigation.

Im Play-Mode wünschten sich die Probanden eine Orientierungshilfe, um $\mathrm{zu}$ wissen, wo sie sich in der virtuellen Umgebung befinden. Hier ist über ein 3D Menü in der virtuellen Umgebung nachzudenken, welches den Grundriss anzeigt (mehr in Kapitel 8). Die Nutzung des kompletten Systems wurde nicht als anstrengend empfunden und es trat keine Art von Cybersickness auf.

\section{$5 \quad$ Fazit}

Der Test zeigte, dass ein gewisses Vorwissen in der Handhabung von gängigen Interaktionsmethoden im Computerbereich notwendig ist, um dieses Interface nutzen zu können. Ohne dieses Vorwissen fehlen die nötigen Grundlagen, um ein solch komplexes Zusammenspiel zwischen 2D- und 3D Interfaces erfassen und auf Anhieb nutzen zu können. Zudem sollte eine Terminologie in Bezug auf die komplette Anwendung festgelegt werden, um Missverständnisse zu vermeiden und die Kommunikation von Anfang an zu verbessern.

Die Usability-Tests haben gezeigt, dass die Entwicklung des Interfaces in die richtige Richtung geht. Insgesamt wurden sehr viele Erkenntnisse gesammelt, in wie weit die Bedürfnisse der Zielgruppe erfüllt wurden. Es entwickelten sich immer wieder Situationen, in denen das Interface in den Hintergrund trat und die kunsthistorische Diskussion und das Erscheinungsbild der Villa in den Vordergrund rückten. Das Interface störte die kollaborative Betrachtung der virtuellen Umgebung nicht, sondern unterstützte sie vielmehr. Die Navigationsform lies 
den Anwendern die Freiheit, sich nicht darauf konzentrieren zu müssen, nicht gegen die nächste virtuelle Wand zu stoßen, sondern teilte die Interaktion in die Planungsphase und die Betrachtungs- und somit Diskussionsphase. Die Usability-Tests unterstützten die Entwicklung des Interfaces enorm. Durch ständige Fehlersuche und -beseitigung, Evaluation des Funktionsumfangs und die wirkliche Nutzung durch die Zielgruppe konnte die Entwicklung in diesem Maße vorangetrieben werden. Die Usability-Tests gaben der Entwicklung einen Rahmen und beeinflussten auch die Unterteilung des Entwicklungsprozesses in die nötigen Phasen.

\section{Literatur}

Fraunhofer IAIS: TwoView/L-Shape, 〈URL: http://www.iais.fraunhofer.de/ 645.html?\&L=0 - Zugriff am 16.12.2008.

Kastenholz, Daneil: 3D-Pathfinding, München 2006 - Technischer Bericht.

Kuck, Roland et al.: Improving the AVANGO VR/AR Framework: Lessons Learned, in: Schumann, Marco/Kuhlen, Torsten (Hrsg.): Virtuelle und Erweiterte Realität: 5. Workshop der GI-Fachgruppe VR/AR, Aachen 2008.

Lester, Patrick: A* Pathfinding, 〈URL: http://www.policyalmanac.org/games/ aStar'Tutorial.htm $\rangle$ - Zugriff am 21.09.2007. 



\section{Cornelius Weidner/Rolf Kruse}

\section{Die Begehung virtueller Realitäten}

Das Projekt,Empty Museum - full of experiences‘ zur Begehung Virtueller Realitäten entstand im Rahmen einer dreimonatigen Bachelorarbeit im Studiengang Media Production an der Hochschule Darmstadt unter der Betreuung von Prof. Dr. Ralf Steinmetz und Dipl.-Ing. Rolf Kruse.

Das Grundszenario für die Begehung ist ein leerer, realer Raum in dem unterschiedliche Inhalte virtuell präsentiert und dadurch räumlich erlebbar werden; es verbindet die Vorteile eines realen Museumsbesuches mit der Individualität einer Online-Präsentation. Klassische Ausstellungen bringen den Besuchern als Selbstverständlichkeit eine räumliche Erfahrung. Durch das umhergehen und umblicken entdecken diese die Ausstellungsobjekte und Ausstellunsräume in ihrer jeweiligen Perspektive im Raum. Online-Präsentationen oder allgemeine Ausstellungen im Netz ermöglichen eine individuellere inhaltliche Wahl. Die schnelle Anpassung der Inhalte oder sogar der spontane Wechsel von Ausstellungen ist den Wünschen des Benutzers überlassen.

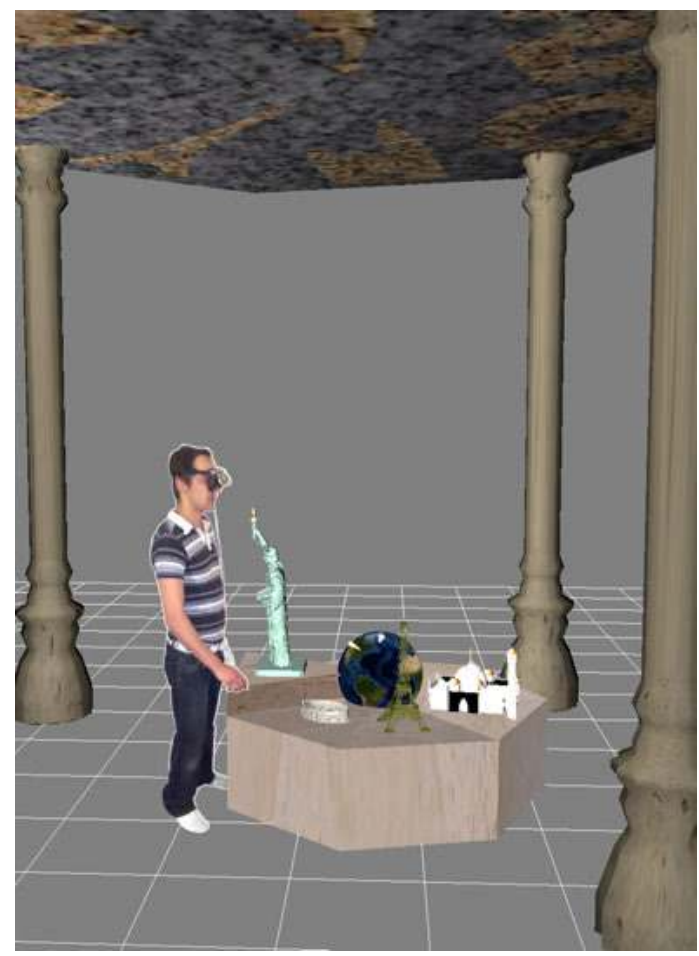

Abbildung 1: Realer Besucher im virtuellen Raum 
Das Projekt „Empty Museum“ zielte auf die Realisierung von Navigationsund Interaktionsprinzipien ab. Ohne zusätzliches Eingabegerät (free-hand) soll die Intraktion allein durch die physische Bewegung des Besuchers innerhalb des realen Raumes steuerbar sein. Dafür waren konzeptionelle Überlegungen zur prototypischen Umsetzung notwendig.

\section{Virtual Reality Technologie}

Der technische Kreislauf zur Begehung setzte sich folgendermaßen zusammen (Abb. 2):

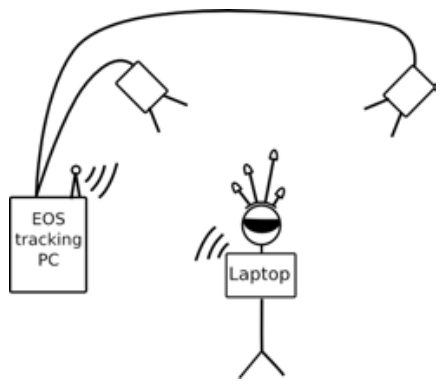

Abbildung 2: Skizze vom technischen Aufbau

Für das Head-Tracking wurde ein optisches 6-DOF-Tracking-System eingesetzt: Zwei Infrarot-Kameras erfassen einen Rigid-Body auf dem Kopf des Besuchers und übertragen die berechnete Position und Orientierung per WLAN an das Render-Notebook. Dieses trägt der Besucher auf dem Rücken und kann sich somit unbeschwert und frei bewegen. Die Stereo-Bilder werden in Echtzeit von Virtools 4 berechnet und auf einem Consumer-HMD als Ausgabemedium am Kopf des Besuchers wiedergegeben.

\section{Dramaturgie - der Benutzer wird zur Besucherln}

Hintergrund der gewählten Architekturszene war die (inoffizielle) Wahl der neuen 7 Weltwunder. Als aktuelles Ereignis fiel das Finale zur Wahl der Weltwunder auf den gleichen Zeitraum wie die Bachelorarbeit und beeinflusste somit die Wahl der auszustellenden Monumentalbauten. Die Entscheidung, allgemein bekannte Monumentalbauten der Neuzeit auszustellen sollte zeigen, daß virtuelle Ausstellungen räumlich größer dimensioniert sein können als reale. Die Wände der Realität stellen kein sichtbares Hindernis im virtuellen Raum dar und können somit ausgeblendet werden. Die Begehung (Abb. 3) gliedert sich in eine Willkommen-Szene, die Auswahlszene des zu begehenden Weltwunders 
und die konkrete Begehung der Weltwunder. Zentraler Teil der Arbeit war die Entwicklung von Prinzipien, zum Übergang zwischen den Szenen und der Auswahl von Teilszenen.

Die Herausforderung bestand in der Realisierung von intuitiven Interaktionsmechanismen, welche die Veränderungen des (virtuellen) Raumes und der enthaltenen Objekte allein auf Grund von Informationen über Position und Blickwinkel entlang der Zeit ermöglichen. Vergleicht man hierbei die Navigation im Internet durch Maussteuerung und die räumlichere Navigation in Videospielen mit Hilfe von Gamepads, so erscheint das gehen und umsehen ohne Eingabemedium sehr einfach. Außerdem sollte sich bei hoher Immersion nicht der Raum um den Besucher bewegen, sondern der Besucher im Raum mit einer auf ihn reagierenden Umgebung; so reagieren Auswahlobjekte z.B. beim „Chable“-Prinzip auf Sichtbereich und Distanz in Abhängigkeit zu Abstand und Blickrichtung des Besuchers: Sie bieten sich dem Besucher an, indem sie ihre Position verändern und sich vergrößern.

\section{Interaktionsprinzipien}

In Anlehnung an Web-User-Interfaces werden in einer weiteren Navigationsszene Auswahlelemente auf dem Boden dargestellt (Abbildung 4 2.v.R.). Ein Element wird ausgewählt, indem sich der Besucher darauf stellt.

Eine weitere Navigationsszene ist der virtuelle Ausstellungsraum. Er besteht aus einer Ansammlung von zweidimensionalen Vorschaubildern, beispielsweise an den virtuellen Wänden, welche auf mögliche Ausstellungsobjekte verweisen. Nähert sich der Besucher einem virtuellen Bild und betrachtet dieses, so taucht das jeweilige 3D-Objekt in der Mitte des Raumes auf und meldet akustisch sein erscheinen.

Die gezielt eingesetzte Überlagerung der virtuellen mit der realen Räumlichkeit bedarf einer geschickten Anpassung. Passt der reale Raum nicht in die Räume der virtuellen Welt, so müssen aus dem Alltag oder der Fiktion bekannte Navigationsmetaphern wie Fahrstühle, Teleporter, Sieben-Meilen-Stiefel oder ähnliche Manipulationen situationsbedingt eingesetzt werden.

Die starke Immersion in der virtuellen Realität und somit das fortschreitende Ausblenden der wirklichen Realität mit ihren Objekten verlangt eine Kollisionserkennung für reale Objekte. Um echte Zusammenstösse der Nutzer mit Raumbegrenzungen zu vermeiden, werden Repräsentanten bei zunehmender Nähe lagerichtig in die virtuelle Szene eingearbeitet (Abb. 5). Ebenfalls werden andere Besucher als abstrahierte Avatare in der virtuellen Umgebung repräsentiert, so dass eine Kollision umgangen und der soziale Faktor erhalten bleibt. 


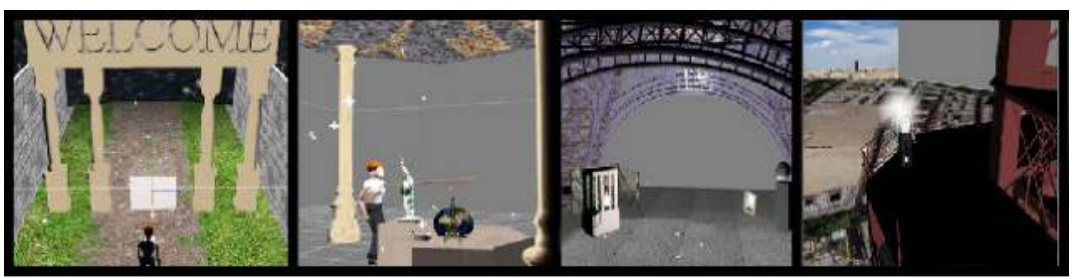

Abbildung 3: Realisierte Szenen: Willkommensszene, Auswahl des Weltwunders, Begehung unter dem Eiffelturm, Begehung der Aussichtsplattform

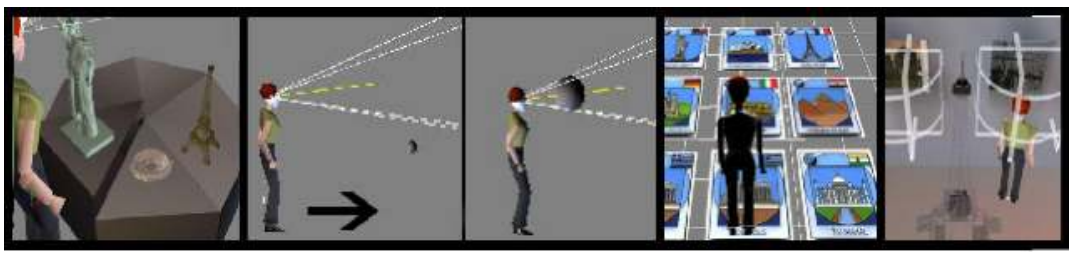

Abbildung 4: Navigation im Virtuellen Raum: Auswahltisch, sich anbietendes Objekt, Bodennavigation, Wand mit zweidimensionalen Vorschaubildern

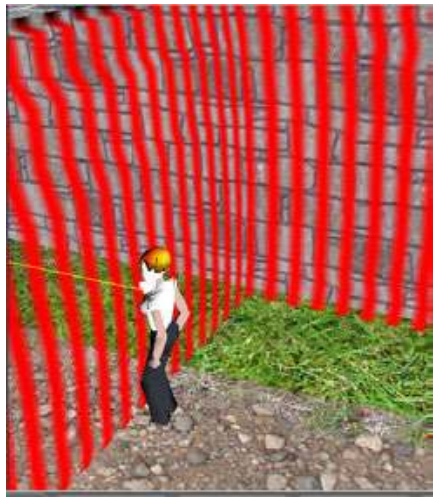

Abbildung 5: Kollisionserkennung der Realität im virtuellen Raum 


\section{$4 \quad$ Erste Tests}

Erste Vorführungen zeigen, dass es sich bei einer solchen Begehungen um eine intensive Erfahrungsmöglichkeit handelt. Das System bietet durch interaktive Simulation neue Erlebnismöglichkeiten von Räumlichkeiten, beispielsweise zerstörte Architektur aus der Vergangenheit, fantastische oder visionäre Gebäude. Grenzenlose Flexibilität unabhängig von Raum und Zeit bieten fantastische Erlebnismöglichkeiten. Technische Probleme bereitete beim Prototypen die geringe Reichweite $\left(6 \mathrm{~m}^{2}\right)$ der begehbaren Fläche und die noch geringe Performance von etwa 6 Bildern pro Sekunde. Jedoch reichte es aus um erste Erfahrungen mit öffentlichem Publikum zu sammeln. Es gab in den Räumlichkeiten immer nur eine Person, welche die Technik zur Begehung mit sich trug und eintauchen konnte. Der Rest vom Publikum konnte dabei nur von aussen zusehen wie sich der Besucher umsah und durch seine Brille scheinbar eine andere Umgebung wahrnahm. So hatten die Zuschauer trotz fehlender Visualisierung (oder gerade wegen) sichtbare Freude daran, direkte Erfahrungen des Besuchers direkt aus der Virtuellen Realität berichtet zu bekommen. Er berichtete live aus einer anderen Welt, während er physisch nur wenige Meter entfernt stand. 



\section{Severin Todt/Christof Rezk-Salama/Andreas Kolb}

\section{Virtuelle Rekonstruktion und interaktive Exploration der Schlossanlage Dillenburg}

Die vorliegende Arbeit präsentiert die Ergebnisse des interdisziplinären Projektes zur dreidimensionalen Rekonstruktion der Festungsanlage Dillenburg, die im Jahre 1768 vollständig zerstört wurde. Für die interaktive virtuelle Erkundung des Schlosses wurde in enger Zusammenarbeit zwischen ortsansässigen Historikern und Wissenschaftlern des Bereichs Computergraphik auf Basis historischer Textdokumente, Zeichnungen und Grundrisspläne ein hochqualitatives 3D Modell entwickelt. Mit dem Modell als Grundlage wurden Computeranimationen und zusätzliche historische Dokumente zu einem DVD Multimediaprojekt zusammengestellt, um einen einfachen Zugang zu der virtuellen Festung zu realisieren. Für die Ausstellung in den Räumlichkeiten des Museums Dillenburg wurde eine Echtzeit-Anwendung entwickelt, die eine virtuelle Begehung des Modells vor Ort ermöglicht. Ausgestattet mit einem Touch-Screen zur Interaktion und einem Großbild-Anzeigesystem sind die Besucher des Museums in die Lage versetzt, die virtuelle Rekonstruktion zusammen mit Zusatzinformationen intuitiv gemäß ihren Bedürfnissen zu erforschen. Mit der Multimediainstallation präsentieren wir eine neue museale Erfahrung, die es dem Besucher des Museums ermöglicht, die historische Stätte gemäß seiner Präferenzen zu erforschen und die jüngere Museumsbesucher animiert, sich mit der Geschichte auseinanderzusetzen.

\section{$1 \quad$ Einleitung}

Multimediaanwendungen sind zu einem essentiellen Bestandteil zur Informationsvermittlung in musealen Umgebungen geworden. Insbesondere interaktive Präsentationen motivieren den Besucher, sich auf neuen Wegen mit dem präsentierten Kulturerbe auseinanderzusetzen. Die Präsentation virtueller Modelle, archäologischer Artefakte, Monumente oder archäologischer Ausgrabungsstätten als interaktive Anwendung, ermöglicht es dem Besucher jegliches Kulturerbe frei nach seinen eigenen Interessen zu erforschen. Je mehr Freiheitsgrade dem Besucher im Rahmen dieser virtuellen Exploration zur Verfügung stehen, desto faszinierender wird das multimediale System empfunden. Umfassende Interaktionstechniken jedoch sind eng verbunden mit komplexen Interaktionsparadigmen und kostentintensiven Multimedia Ausstattungskomponenten. State-Of-The-Art Technologien sind daher zu einem wichtigen Bestandteil moderne Multimediainstallationen in Museen geworden.

Die Anwendung aktueller Technologien auf historische Daten hat das Potential jüngere Generationen zu motivieren, das Interesse an den Kulturschätzen 


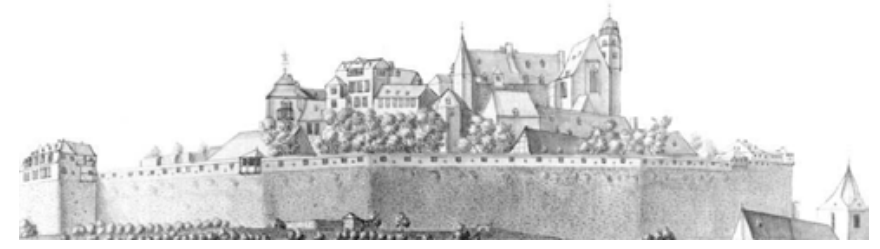

Abbildung 1: Die Festungsanlage Dillenburg vor der Zerstörung, illustriert von Catharina Helena Dörrien im Jahre 1760.

unsere Ahnen zu steigern. Auf der anderen Seite jedoch können Computertechnologien eine Hürde darstellen für ältere Generationen von Besuchern die nicht mit aktuellen Interaktionstechniken vertraut sind. Das perfekte Multimediasystem berücksichtigt die Aspekte beider Benutzergruppen. Solch ein System bietet ein duales Interaktionsparadigma, das Inhalte für untrainierte Besucher leicht und komfortable zugänglich macht, bietet gleichzeitig jedoch zusätzlich die Möglichkeit komplexerer Explorationstechniken für erfahrenere Benutzergruppen.

Diese Arbeit präsentiert die Ergebnisse unseres Projektes, das die virtuelle Rekonstruktion der Festungsanlage Dillenburg zum Ziel hatte. Eine der einzigartigen Herausforderungen dieses Projektes bestand in der Tatsache, dass lediglich sehr wenige Überreste der Festungsanlage heute noch existieren und für die Rekonstruktion Verwendung finden konnten. Daher basiert die Rekonstruktion in der Hauptsache auf textuellen Beschreibungen, historischen Zeichnungen und groben Skizzen des Schlosses. Als Beispiel ist in Abb. 1 die detaillierteste verfügbare historische Ansicht der Anlage dargestellt, gezeichnet im Jahre 1760 von der Biologin Dörrien unmittelbar vor der Zerstörung. Trotz der Detailtreue der vorliegenden Zeichnung stehen viele Aspekte dieser Zeichnung im Widerspruch $\mathrm{zu}$ anderen historischen Dokumenten verschiedener Quellen.

Die dreidimensionale Rekonstruktion der Anlage wurde in enger Zusammenarbeit mit den Historikern des örtlichen Museums Dillenburg manuell mit Hilfe eines 3D Modellierungsprogramms auf Basis historischer textueller Aufzeichnungen, Zeichnungen und erhaltener Grundrisspläne erstellt. Neben der 3D Rekonstruktion umfasst dieses Projekt weiter die Entwicklung einer interaktiven Anwendung zur virtuellen Exploration der Schlossanlage Dillenburg, die in die Ausstellung Festungsbau in der frühen Neuzeit im Museum Dillenburg integriert wurde. Die Multimediainstallation besteht aus einem intuitiven Interaktionsdevice, das zur Echtzeit-Interaktion in der dreidimensionalen Rekonstruktion genutzt wird, die mit Hilfe eines Großbild Display-Systems präsentiert wird.

Zur Umsetzung der interaktiven Navigation wurde ein Touch-Screen eingesetzt, um untrainierten Besuchern einen einfachen Zugang zu den Inhalten zu 
gewähren und komplexerer Navigationsmöglichkeiten für eine tiefere Exploration zu ermöglichen. Das präsentierte Modell kann mit Hilfe des Touch-Screen ohne Beschränkungen interaktiv erforscht werden und ist über Menüpunkte im Display Menü zusätzlich direkt als offline Animation verfügbar. Zusätzliches Informationsmaterial, wie textuelle Beschreibungen, Bilder und Erzählungen ist über Informationstafeln aus der interaktiven Anwendung heraus verfügbar. Mit der Präsentation digitaler Zusatzinformationen innerhalb der virtuellen Präsentation wird die Anwendung selbst zu einer virtuellen Ausstellungsfläche.

Im Folgenden ist die Arbeit wie Folgt strukturiert:

Im Abschnitt 2 geben wir einen kurzen historischen Überblick zur Festungsanlage Dillenburg. Abschnitt 3 beschreibt die virtuelle Rekonstruktion der Festungsanlage. Das Rendering und das Compositing der erstellten offline Animationen wird im Abschnitt 4 beschrieben. Das Real-Time Rendering sowie die Mensch-Computer Interaktion werden im Abschnitt 5 im Detail beleuchtet. Im Abschnitt 6 fassen wir diese Arbeit zusammen.

\section{Historischer Hintergrund}

Erstmalig erwähnt im Jahre 1255 diente das Schloss Dillenburg als Siedlungspunkt für die Stadt Dillenburg die ihre Stadtrechte im Jahre 1344 erlangte. Ausgrabungsfunde jedoch lassen den Schluss zu, dass das Schloss bereits im Jahre 1130 erbaut wurde.

Das Schloss wurde im Verlauf der Dernbach Fede in der ersten Hälfte des 14.Jahrhunderts erstmals zerstört. In den Jahren 1453 bis 1473 wurde es wieder aufgebaut und kontinuierlich über die folgenden Jahre erweitert. In dieser Periode erfolgte der Ausbau des Schlosses zu einer Festungsanlage. Die Hohe Mauer, eine Mauer von 300m Länge und 25m Höhe wurde im Jahre 1535 fertiggestellt und die Kirche der Stadt Dillenburg im Jahre 1552 in die Schlossanlage integriert (siehe Abb. 1 für eine Illustration der Festung). Fortlaufend erweitert, stellte die Festungsanlage im Jahre 1583156 Betten, jedes von ihnen doppelt belegt, und beherbergte im Jahre 1600 bis zu 3000 Soldaten.

Während des Dreißigjährigen Krieges (1618 - 1648) wurden weitere zehn 25 Pfund Kanonen und 30 zwölf Pfund Kanonen installiert. Schwarzpulver, ausreichend für 500 Schüsse von 1000 Musketen wurde eingelagert, um die Schlossanlage gegen Angriffe zu stärken. Nicht durch die Kriegsaktivitäten des Dreißigjährigen Krieges beeinträchtigt, war die Festung jedoch einer der Hauptschauplätze des Siebenjährigen Krieges (1756 - 1763) (siehe Abb. 2 für eine Darstellung der Kriegsaktivitäten). Im März 1759 wurde die Festungsanlage für einen kurzen Zeitraum von nur acht Monaten von französischen Kräften eingenommen, bis sie überraschend von Streitkräften in einer Stärke von nur 100 Soldaten zurückerobert wurde. Nur ein halbes Jahr später, ab dem 28. Juni des 
Jahres 1760, wurde die Festung erneut von einer französischen Militärmacht von 3000 Soldaten belagert. Nach 13 Tagen massiver Angriffe, ordnete Hauptmann Düring am 15. Juli das Hissen der weißen Flagge an. Mit der Kapitulation wurde die Festungsanlage den französischen Besatzungsmächten übergeben. Im Verlauf der kommenden vier Jahre blieb die Festung vom französischen Miltitär besetzt und diente als militärische Basis für die französischen Kriegsaktivitäten in Mitteleuropa.

Mit dem Ende des Siebenjäbrigen Krieges verlor Dillenburg für die französischen Streitkräfte an strategischer Bedeutung. Als Folge dessen wurde die Zerstörung angeordnet. Gebäude über Bodenniveau wurden zerstört und geschliffen, unterirdische Verteidigungsanlagen verfüllt, um einem Wiederaufbau vorzubeugen. Aus diesem Grund konnten bis heute in den Ausgrabungen der letzten Jahrzehnte nur wenige Überreste geborgen werden. Seit 1872 wurden verschieden Gebäude auf dem Gebiet der Festungsanlage Dillenburg erbaut, als Ersatz für die imposante Silhouette der Festung. Heutzutage beherbergen diese Gebäude einige der Museen der Stadt Dillenburg.

\section{$3 \quad$ Virtuelle Rekonstruktion}

Aufgrund der vollständigen Zerstörung der Festungsanlage Dillenburg konnten seit den ersten Ausgrabungen im Jahre 1849 nur wenige antike Artefakte geborgen werden. Die bruchstückhaften und teilweise widersprüchlichen his-

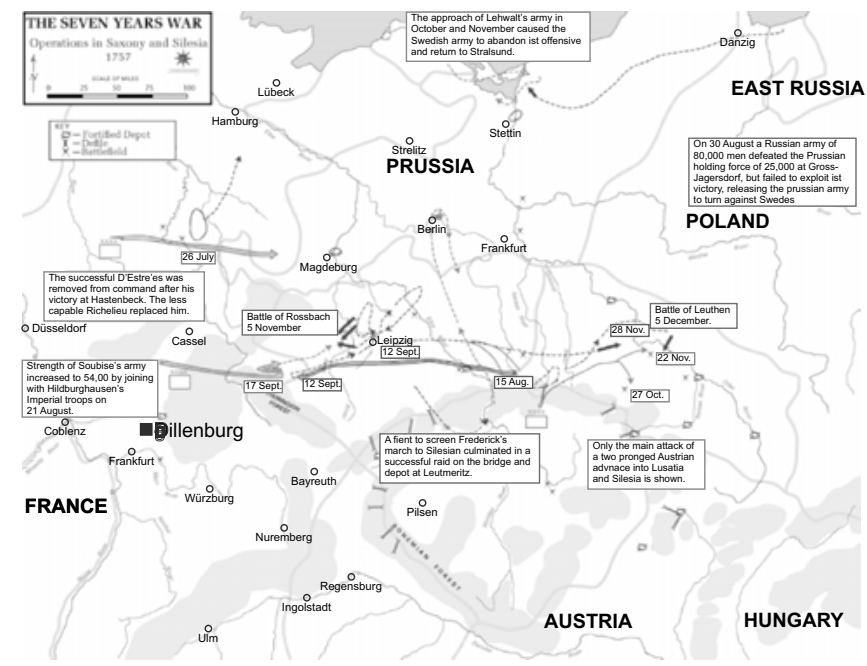

Abbildung 2: Siebenjähriger Krieg - Militärische Operationen, 1757. 
torischen Daten stellten für die Umsetzung der virtuellen Rekonstruktion eine besondere Herausforderung dar. Historische Textdokumente und Zeichnungen die von den Historikern vor Ort über die letzten Jahrzehnte gesichert wurden stellten bei der Rekonstruktion den größten Teil der verwendeten Hintergrundinformationen dar. Auf Basis dieser Dokumente wurde in einem langwierigen iterativem Verfeinerungsprozess unter Zuhilfenahme der 3D Modellierungssoftware Autodesk Maya ${ }^{T M} 8.0$ und in enger Zusammenarbeit mit den Historikern und dem Museum Dillenburg das 3D Modell erstellt. Über den gesamten Verlauf der Rekonstruktion hinweg wurden vorläufige Ergebnisse von den Historikern validiert und bei Bedarf den Details aus Zeichnungen und Textdokumenten angepaßt, um Widersprüche mit vorhandenen Dokumenten und Unsicherheiten weitestgehend auszuräumen.

\subsection{Grundriss Zeichnungen}

Mit der Anordnung der vollständigen Zerstörung der Festung Dillenburg im Jahre 1768 wurde das Schloss in den folgenden Jahren unter dem Kommando des Fähnrichs Pfau zerstört und die Trümmer abgetragen. Glücklicherweise erstellte der Fähnrich noch vor der Zerstörung detaillierte Grundrisspläne aller einzelnen Stockwerke der Festungsanlage (siehe Abb. 3).

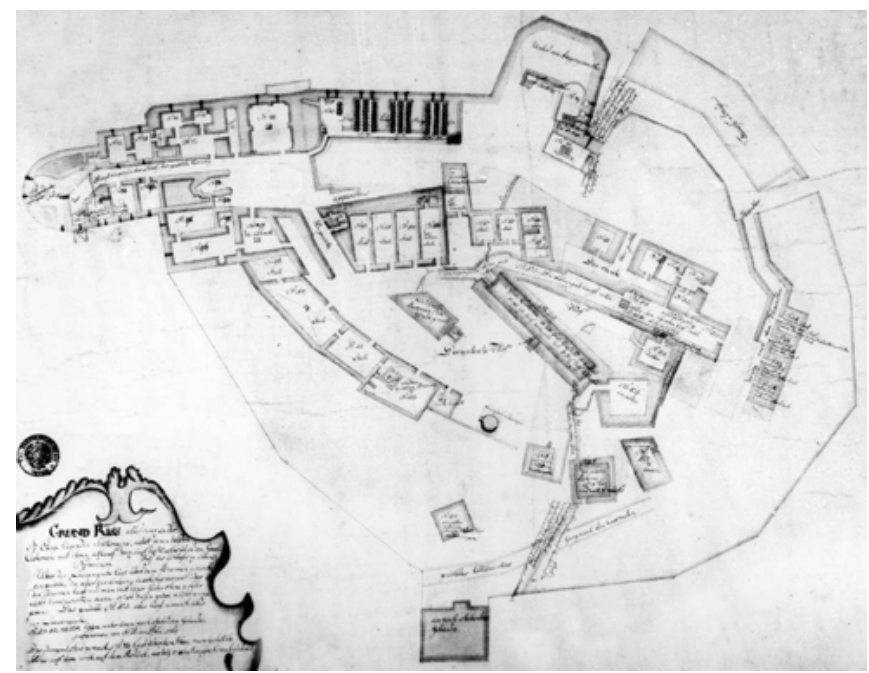

Abbildung 3: Plan des untersten Stockwerkes der Festungsanlage Dillenburg, gezeichnet von Fähnrich Pfau vor der Zerstörung im Jahre 1768.

Mit diesen Aufzeichnungen stehen die Grundrisse der insgesamt fünf Stockwerke zur Verfügung. Sie stellen die verlässlichste historische Quelle zur Positionierung und groben Modellierung der Gebäude dar. Bedauerlicherweise konnten 
zu den Aufzeichnungen des Fähnrichs keine genauen Maßangaben geborgen werden. In sich, jedoch, sind die Aufzeichnungen in hohem Maße konsistent. Die einzelnen Grundrisse konnten so unter Anwendung von quadratischen Optimierungsverfahren zum Abgleich korrespondierender Punkte mit akzeptabler Genauigkeit gegeneinander ausgerichtet werden.

Einzelne Gebäudefundstücke die mit den Ausgrabungen der vergangen Jahre konserviert werden konnten, wurden im Jahre 2005 im Auftrag des Museums Dillenburg amtlich vermessen. Für diese Fundstücke stehen GaußKrüger Koordinaten zur Verfügung. Die Registrierung dieser Fundstücke mit den Grundrissplänen von Pfau stellen den Größenmaßstab für die Pfauschen Pläne und damit eine verläßliche Datenbasis zur Verfügung. Die Registrierung erfolgte auf Basis fünf manuell ausgewählter korrespondierender Punkte, die sich in allen der historischen Pläne identifizieren ließen mit Hilfe eines Least-Square Five-Point Matching Algorithmus. ${ }^{1}$

\subsection{Gebäude-Modellierung}

Die Erstellung der 3D Modelle aller baulichen Konstruktionen erfolgte in zwei Phasen. In der ersten Phase wurde eine grobe polygonale Abschätzung der Gebäude modelliert, um die Lage, die Grundform und die Größe der Gebäude zu bestimmen. Während die Grundfläche, Lage und Größe der Gebäude direkt aus den Grundrißplänen abzuleiten waren, waren keine expliziten Informationen zur Höhe der Gebäude verfügbar. Einzig detaillierte textuelle Beschreibungen der realtiven Höhenunterschiede der Gebäude so wie verschiedene perspektivische Zeichnungen dienten der Bestimmung der Gebäudehöhen.

Die groben Geometrien der ersten Phase dienten den Historikern als Diskussionsgrundlage. Zu diesem Zweck wurden vorläufige virtuelle Ansichten des groben 3D Modells generiert. Erstmals stand den Historikern damit eine visuelle Möglichkeit zur Verfügung, historische Daten einer Richtigkeitsprüfung zu unterziehen, wie sie zuvor nicht möglich war. In einem iterativen Verfahren wurde die Form und die Position individueller Gebäude im Rahmen der verfügbaren historischen Daten verlässlich festgelegt. Die 3D Visualisierung der Modelle erlaubte den Historikern auch für solche Gebäude eine plausible Abschätzung der Gebäudegeometrie zu bestimmen, für die eine umfassende Beschreibung nicht verfügbar war.

In der zweiten Phase erfolgte die detaillierte Modellierung der Gebäude basierend auf der abgeschätzten 3D Gebäudeform. Details der Gebäude, wie die Anzahl der Stockwerke, die Anzahl und die Form der Fenster, Türen und Dachgauben wurden aus perspektivischen Zeichnungen verschiedener Blickrichtungen gewonnen. Diese Informationen dienten der groben strukturellen

1 Nister: An Efficient Solution to the Five-Point Relative Pose Problem. 
Anordnung von Gebäudedetails. Für jedes der Detailelemente wurde vorerst ein Platzhalterobjekt in das Gebäudemodell eingesetzt. Mit Hilfe dieser Platzhalter kann die Detailmodellierung sehr effizient erfolgen.

Für einzelne Gebäudedetails wurde eine Objektdatenbank aufgesetzt. Variationen verschiedener Türen, Fenster und Dachgauben wurden in dieser Datenbank hinterlegt. Jedes individuelle Element konnte so separat modelliert und begutachtet werden. Die im Vorwege in die Gebäude integrierten Platzhalter wurden dann mit einer Modellinstanz aus der Datenbank assoziiert. Spätere Änderungen an diesen Detailgeometrien wurden so automatisch in allen betroffenen Gebäuden übernommen (siehe Abb. 4).

Ein identischer Ansatz wurde ebenfalls auf einer höheren Abstraktionsebene eingesetzt. Die Masterdatei mit der kompletten Festung wurde ebenfalls aus einer Reihe von Objektreferenzen aufgebaut. Anstelle von Instanzen aus der Modelldatenbank wurden mit diesen Referenzen individuelle Dateien referenziert, jede mit dem 3D Modell eines individuellen Architekturteils der Festung. Durch diese Anordnung wurden Änderung an den Referenzdateien automatisch in der Masterdatei übernommen. Die Unterteilung der Gesamtgeometrie in einzelne Teilgeomtrien erlaubte auch hier die individuelle Modellierung, Begutachtung und Festlegung von 3D Modellen. Aufgrund der nicht verfügbaren historischen Angaben zum inneren Erscheinungsbild der Gebäude wurden diese nicht modelliert.

\subsection{Material und Textur Synthese}

Die Rohdaten für die Texturen der Wände wurden von Gebäuden der Festung Altena, einer Archeologischen Stätte der gleichen Epoche mit Baustilen ähnlichen denen der Dillenburg, aquiriert. Im Verlauf des Projektes stellte sich die Synthese von Materialien und Texturen als zeitaufwendiges und komplexes Vorhaben heraus. Idealerweise würden gleiche Materialien durch ein ähnliches, dennoch jedoch nicht identisches Erscheinungsbild zu identifizieren sein. Für einen überzeugenden visuellen Eindruck der Materialien wurde daher spezieller Aufwand betrieben. Nicht texturierte Oberflächenmaterialien konnten relativ einfach durch eine leichte Anpassung der Materialreflektivität oder ambienten Farbeinstellungen variiert werden.

Texturierte Oberflächen waren für variable Auflösungen und Level of Detail des Modells anzupassen, um einen überzeugenden Eindruck an allen Stellen des 3D Modells zu gewährleisten. Eine mögliche Lösung des Problems besteht in der Verwendung prozeduraler Texturen. Prozedurale Texturen können über die Anpassung der Generierungsparameter leicht und sehr flexibel angepaßt und variiert werden. Wie Performance Messungen zeigten, wirkt sich der Einsatz prozeduraler Texturen in hohem Maße negativ auf die Rendering-Performance aus. Da dieses Projekt unter strengen zeitlichen Vorgaben und mit limitierten 

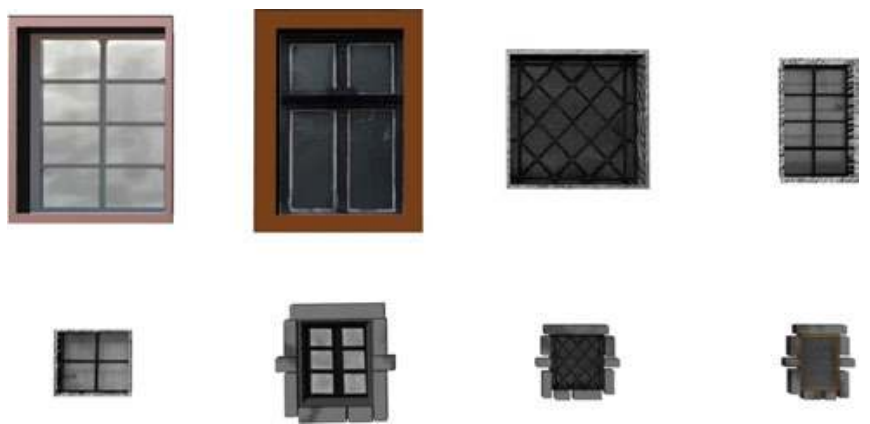

Abbildung 4: Auszug aus der Modelldatenbank - Hier eine Auswahl an Fenstervariationen.

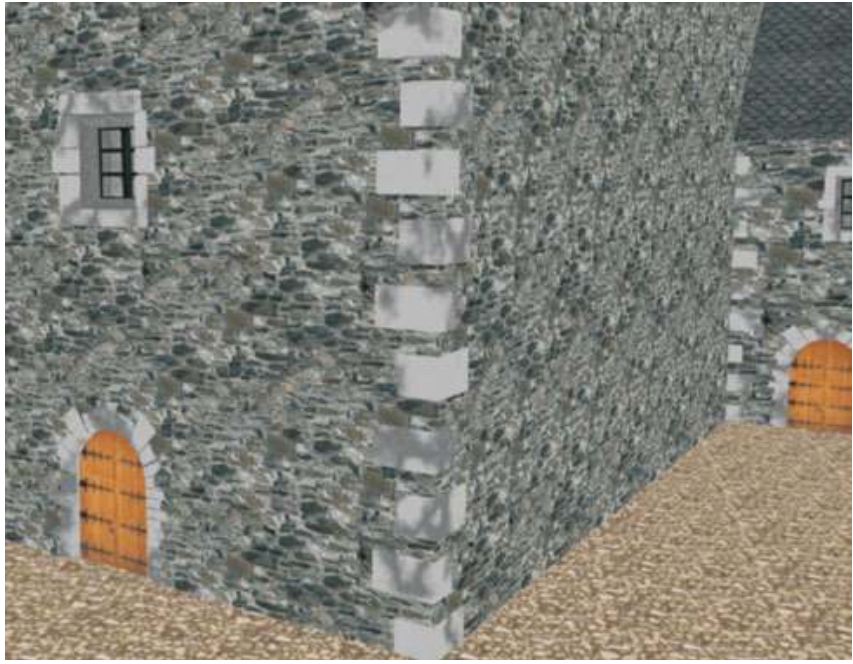

Abbildung 5: Rendering eines Ecksteinsegmentes. Prozedurale Texturen wurden auf einzelne größere Steinsegmente angewandt. Für größere Oberflächen wie Wände kamen Tiled Textures zum Einsatz. 
Ressourcen zu realisieren war, kamen prozedurale Texturen nur an ausgesuchten Stellen zum Einsatz (siehe Abb. 5).

Trotz der vorhandenen Vergleichsarchitektur in der Festung Altena, war die Akquisition hochaufgelöster Texturen für große Teile der zu erstellenden Materialien nicht möglich. Stattdessen wurden Texturen für einzelne Steine aus verschiedenen Mauerstilen akquiriert, die dann zu Tiled Textures zusammengefügt und auf große Areale des Dillenburg 3D Modells angwendet wurden. Die direkte Verwendung von Tiled Textures führt zu einer Erzeugung von störenden wahrnehmbaren visuelle Wiederholgunsstrukturen. Die Anordnung von Tiled Textures unter Verwendung spezieller Algorithmen wie Wang Tiles ermöglicht die Vermeidung solcher Wiederholungsartefakte. Diese Techniken sind jedoch nicht direkt in der von uns verwendeten 3D Modellierungsumgebung Maya verwendbar und bedürfen spezieller Methoden zur Texturakquisition. ${ }^{2}$

Mit Bricks 'n Tiles kam stattdessen ein Textur Synthese Tool zum Einsatz, das die effiziente Generierung großer Texturen von nur einem einzigen kleinen Ausschnitt der Textur ermöglicht. ${ }^{3}$ Die mit dieser Technik erstellten Texturen zeigen keine visuellen Wiederholungsartefakte für Nahaufnahmen. Für Aufnahmen die mit einer Panorameinstellung erfolgen, können Wiederholung in Texturen aufgrund von Speicherlimitierungen nicht ausgeschlossen werden. Trotz dieser Einschränkungen für Panoramaaufnahmen kam diese Technik erfolgreich für die erste Version dieses Projektes zum Einsatz, da ein Großteil der zur Generierenden Aufnahmen als Nahaufnahme angelegt wurde.

\subsection{Umgebungsmodell}

Das Umgebungsmodell konnte automatisch auf Basis vorliegender Datensätze der (Hessischen Verwaltung für Bodenmanagement und Geoinforamtion, HVBG) erstellt werden. Zu diesem Zweck wurde ein (Digitales Geländemodell DGM25) des Standortes aus dem Datensatz extrahiert. Für diesen Datensatz standen absolute Höhenangaben für jeden Gitterpunkt eines Gitters mit einer Gitterweite von $25 m$ zur Verfügung. Weiter waren jedem Gitterpunkt eindeutige Gauß-Krüger Koordinaten zugewiesen. Mit Hilfe dieser Koordinaten konnte der verfügbare Datensatz mit den amtlichen Vermessungen der Archeologischen Artefakte der Dillenburg und damit mit dem erstellten 3D Modell registriert werden (siehe Abschnitt 3.1). Die Erzeugung der 3D Repräsentation des Umgebungsmodells erfolgte durch eine automatische Überführung des Höhenfeldes in eine triangulierte und verfeinerte polygonale 3D Struktur.

2 Cohen et al.: Wang Tiles for image and texture generation

3 Begand: Bricks 'n Tiles. 


\section{$4 \quad$ Rendering}

Zur Durchführung des Rending wurden alle Referenzen der Masterdatei (siehe Abschnitt 3.2) aufgelöst und sämtliche Materialien, Texturen und Shaderelemente in dieser Datei zusammengeführt. Die resultierende Szene beinhaltet alle Gebäude, jedes von Ihnen als separates Objekt, das Umgebungsmodell, eingefügte Vegetation sowie die zur Ausleuchtung der Szene gesetzten Lichter. Die finale Version der Szene wurde ein letztes Mal begutachtet. Redundante Polygone und nicht genutzte Materialien und Texturen wurden entfernt, um die Szenenstruktur für beste Rendering Performance zu optimieren. Abschließend wurde ein Sky Dome, repräsentiert durch eine polygonale Hemisphere und texturiert mit einer Fischaugen-Aufnahme eines leicht bewölkten Himmels, eingefügt. Die Lichtquellen wurden gemäß der Hauptlichtrichtung der Himmelfotografie angepaßt. Die finale Version der Szene bestand aus 1.78 Millionen Polygonen und $66 \mathrm{MB}$ an Texturdaten. Das Rendering erfolgte in einem verteilten Prozess auf einem PC Cluster mit bis zu 15 Renderknoten. Insgesamt wurden mit Hilfe des Renderers Mental Ray for Maya über 24000 Bilder gerendert.

\subsection{Kamera Einstellungen and Animation}

Für das entgültige DVD Produkt wurden 13 verschiedene Kameraanimationen gerendert, jede von ihnen mit unterschiedlichem Kamera-Bewegungspfad. Der Rundflug durch die virtuelle Rekonstruktion, eine acht-minütige Animation, repräsentiert den Hauptteil der Animationen. Zwölf zusätzliche Kameraanimationen zeigen Details einzelner Gebäude und ergänzen den Rundflug.

Für eine homogenes Erscheinungsbild waren für alle der zu generierenden Animation identische Kameraeinstellungen passend zu allen Variationen der Bewegungspfade der Kamera zu wählen. Für die Aufnahmen wurde ein weiter Öffnungswinkel gewählt, um die komplette Ausdehnung der Gebäude erfassen zu können. Trotz der zu beobachtenden leichten Verzerrungen für Aufnahmen mit großem Öffnungswinkel haben sich diese Einstellungen zur Demonstration der Größe und Mächtigkeit der Festungsanlage als ideal erwiesen.

Für das finale Rendering wurde Mental Ray for Maya eingesetzt. Für diesen Renderer musste der ideale Kompromiss zwischen Rendering Performance und Bildqualität bestimmt werden. Die Evaluation des Renders hat eine maximale Reflektionstiefe von zehn Reflektionen, zehn Lichtbrechungen und eine maximale Tracetiefe von 20 als für unseren Produktionsprozess optimal identifizieren können.

Die Kamerapfade wurden unter Zuhilfenahme von Pfad-Animationen festgelegt. Pfad-Animation bieten im Vergleich zu Keyframe-Animationen eine Vielzahl von Vorteilen. Mit Pfad-Animationen steht eine direkt modellierbare visuelle Repräsentation des Bewegungspfades zur Verfügung. Der Animations- 
fluss kann mit einfachsten Hilfsmitteln über die Anpassung der Bewegungsgeschwindigkeit entlang des Pfades, die Anpassung der Blickrichtung und die einfache Änderung des Pfades angepaßt werden.

\subsection{Compositing}

Das Rendering der Szene erfolgte in mehreren verschiedenen Render-Passes für eine effiziente digitale Bearbeitung unter Verwendung von Adobe After Effects. Diese Strategie zeigt ihre Vorteile insbesondere in der Aufteilung visueller Attribute in verschiedene Layer. Die Helligkeit und das visuelle Erscheinungsbild der Szene kann in der Nachbearbeitung angepaßt werden, ohne dass ein erneutes Rendering der Szene notwendig wird.

Um ein höchstes Maß an Flexibilität zu erzielen, wurden fünf verschiedene Layer erzeugt (siehe Abb. 6):

1. Der Ambient Pass zeigt die Basisfarbe individueller Objekte mit den Texturen, jedoch ohne Beleuchtung, Reflektionen oder Schatten. Der Renderer berechnet dazu lediglich den ersten Schnittpunkt des aktuellen Sehstrahls mit der Geometrie und bestimmt für diesen die Basisfarbe an der Oberfläche.

2. Ambient Occlusion ${ }^{4}$ beschreibt eine einfache und dennoch sehr überzeugende Abschätzung der globalen Beleuchtung an einem Oberflächenpunkt und findet in der digitalen Produktion vielfache Anwendung. Für den Ambient Occlusion Render Pass werden ausgehend von einem Oberflächenpunkt Strahlen in jede Richtung der mit dem Punkt assoziierten Hemisphere evaluiert. Die Helligkeit des Oberflächenpunktes wird dann aus dem Prozentsatz der Strahlen berechnet, die keine anderen Teile der Geometrie schneiden.

3. Ein separater Shadow Pass berechnet die Schatten die von denen in der Szene platzierten Lichtquellen geworfen werden.

4. Spekulare Reflektionen und Highlights die aus direkter lokaler Beleuchtung resultieren werden in einem separaten Specular Pass berechnet.

5. Zuletzt wird in dem Depth Layer pro Pixel die Tiefeninformation für das gerenderte Bild bestimmt. Da die Tiefe jedoch bereits aus dem ersten Renderschritt, dem Ambient Pass bestimmt werden kann, ist für diesen Layer kein gesonderter Render Pass notwendig.

Für das letztendliche Bild werden alle erzeugten Layer kombiniert. Der Ambient Occlusion Layer wird als Faktor für den Ambient Layer verwendet und

4 Landis: Production-Ready Global Illumination; Zhukov/Iones/Kronin: An Ambient Light Illumination Model. 

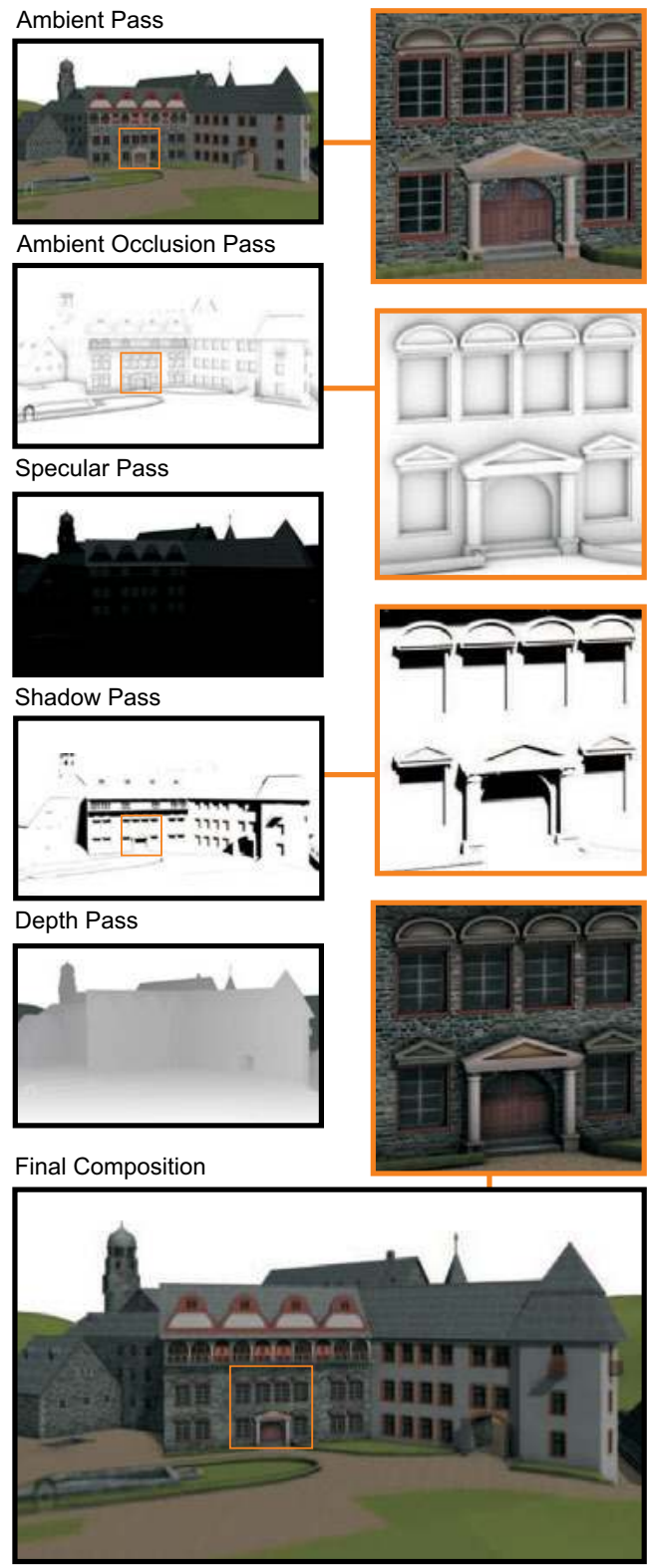

Abbildung 6: Die endgültige Darstellung der Szene ergibt sich aus dem Compositing der fünf einzelnen Renderings der separat gerenderten Passes.

resultiert in Verdunkelungseffekten an Kanten und tiefen Strukturen. Spekulare Highlights und Reflektionen werden sukzessive addiert. Der Shadow Layer wird 
mit diesem Resultat geblendet. Das dabei verwendete Blendgewicht erlaubt explizite Kontrolle über die Abdunkelung in den Schattenbereichen. Der Depth Layer findet zuletzt zur Erzeugung von Tiefeneffekten Anwendung.

\section{Interaktive Exploration}

Mit der erzeugten DVD steht ein traditionelles Medium zur Präsentation der rekonstruierten Festungsanlage zur Verfügung. In Kooperation mit dem Museum wurde das Projekt erweitert, um eine interaktive Exploration zu realisieren. Es wurde aktuelle Echtzeit Graphikhardware eingesetzt, um einen interaktiven Rundgang durch die virtuelle Festungsanlage Dillenburg zu realisieren. Dieser Abschnitt beschreibt die wichtigsten Aspekte bezüglich des Design und der Umsetzung eines solchen Systems.

\subsection{Echtzeit Rendering}

Moderne interactive 3D Anwendungen kombinieren eine große Vielfalt an Technologien wie Szenenmanagement, realitätsnahe Graphik mit überzeugenden Licht- und Schatteneffekten, Kollisionserkennung, Navigation und Interaktion. Im Rahmen dieses Projektes konnte diese Vielfalt an Technologien nicht von Grund auf neu umgesetzt werden. Aus der Open-Source Community stehen jedoch eine Vielzahl von 3D Render Engines zur Verfügung, die sich für dieses Projekt einsetzen lassen.

Nach der Evaluierung verschiedenster Open-Source Projekte, wurde eine der populären 3D-Engines names $O G R E^{5}$, kurz für "Object-Oriented Graphics Rendering Engine”, als optimale Lösung für dieses Vorhaben identifiziert. Diese Render-Engine stellt für fast alle der von uns definierten Anforderungen eine Lösung bereit. OGRE überzeugt durch eine große Benutzergemeinde, sehr aktive Foren und Wiki Webseiten, die, wann immer nötig, den nötigen Support bieten. Obwohl einige unsere Anforderungen, wie zum Beispiel die Bereitstellung eines Sound-Managers nicht direkt geleistet werden, bleibt die Render-Engine dank des objektorientierten Designs leicht um zusätzliche Funktionalitäten zu erweitern und anzupassen.

\subsection{Konvertierung der Szene}

Nach der Wahl der 3D Render-Engine begann die Konvertierung der Szene in echtzeitfähige Formate. Der mit OGRE verfügbare Maya-Exporter ermöglichte die effiziente Konvertierung aller Geometrien. Alle polygonalen Strukturen und Materialien der einzelnen Gebäude, auch die Decal-Texturen wurden in das native OGRE Format überführt. Die Größe der exportierten Geometrien und

5 Ogre 3D: Open Source Graphics Engine. 
Texturen machte ein Rendering in Echtzeit und damit die flüssige Interaktion unmöglich. Aus diesem Grund wurde das gesamte Modell einer Revision unterzogen, mit dem Ziel, hochdetaillierte Elemente händisch zu vereinfachen.

Für die interaktive Navigation durch die virtuelle Festungsanlage wurde zusätzlich eine vereinfachte Kollisionsgeometrie erzeugt. Die Kollisionsgeometrie wurde in der Folge in OGRE und in Verbindung mit dem Plugin zur Kollisionserkennung eingesetzt.

Gefolgt vom erfolgreichen Import der Szenengeometrie, war eine passende Beleuchtungsstrategie zu entwickeln. Während die offline Animationen mit Mental Ray Raytracing Technologien gerendert wurden, mussten für das Echtzeitrendering neue Oberflächenshader entwickelt werden, um dem visuellen Eindruck der offline Animation möglichst nahe zu kommen. Die Entscheidung viel auf die Umsetzung der gesamten Beleuchtung mit Hilfe bildbasierter Beleuchtung und vorberechneter Texture Maps.

Unsere Beleuchtungstechnik basiert auf einer Reihe von Environment Maps die mit verschiedenen spekularen Exponenten vorgefiltert wurden. ${ }^{6}$ Gloss Maps beschreiben die Reflektivität einer Oberfläche. Normal Maps kommen zur Modellierung von rauen Oberflächen und kleinen Oberflächenstrukturen zum Einsatz. Mit diesen Techniken kann realitätsnahe lokale Beleuchtung in Echtzeit erfolgen.

Globale Beleuchtungseffekte und Schatten konnten ohne signifikanten Einfluss auf die Rendering Performance hinzugefügt werden. Sämtliche Schatteninformationen wurden zu diesem Zweck unter Verwendung von Maya's Baking Funktionalität vorberechnet. Das Baking erlaubt die einfache und schnelle Erstellung von Texturen mit Beleuchtungsinformationen. Solche Texturen wurden für die im Abschnitt 4.2 beschriebenen Ambient Layer und den Shadow Pass erzeugt. Schon die Kombination nur dieser zwei Texturen resultiert in einem sehr überzeugendem visuellen Eindruck. Abb. 8 zeigt das finale Rendering, hier mit überblendeten Navigationselementen.

\subsection{Benutzer Interaktion}

Die zum Einsatz kommende Interaktionstechnik muss verschiedenen Benutzergruppen einen einfachen Zugang zu den 3D Inhalten ermöglichen. Benutzergruppen des Museums Dillenburg unterscheiden sich in großen Teilen bezüglich des technischen Hintergrundverständnisses und ihrer Möglichkeiten und Erfahrungen mit der Mensch-Computer unterscheiden sich weiter durch ihr Interesse an historischen Daten und ihren Enthusiasmus für Kulturschätze. Aus diesen Gründen ist die Interaktionstechnik so zu gestalten, dass sie für die untrainierten Besucher einfach zu bedienen ist und dennoch ausreichend komplex ist, um bei

6 Kautz/McCool: Approximation of Glossy Reflection with Prefiltered Environment Maps. 
weniger interessierten Besuchern das Interesse an der virtuellen Ausstellung zu wecken.

\section{Interaktionsdevice}

Um den verschiedenen technischen Qualifikationen der Besucher gerecht zu werden, muss ein Interaktionsdevice sowohl flexibel als auch einfach zu bedienen sein. Das Device muss robust sein und böswilligen Beschädigungen widerstehen. Es muss intuitiv in der Benutzung sein aber gleichzeitig umfassende Interaktionsmöglichkeiten bieten. Letztlich muss sich das Gerät dezent in die museale Ausstellung integrieren.

Die im täglichen Gebrauch befindlichen Devices Mouse und Tastatur bieten das höchste $\mathrm{Maß}$ an Flexibilität, sind jedoch stark anfällig gegen Manipulationen. Es ist schwierig sie dezent in die Ausstellung zu integrieren und ihre Handhabung wird schnell zu komplex, wenn die Interaktion eine einfache Selektion mit der Mouse und einfache Texteingaben überschreitet. Joystick, 3D-Mouse oder Trackball bieten eine intuitivere Handhabe, finden bei älteren Besuchern im alltäglichen Leben jedoch nur in Ausnahmen Anwendung. Sie sind weiter beschränkt in ihrer Flexibilität und benötigen in den meisten Fällen weitere Erklärungen oder Einweisungen, um eine reibungslose Benutzung zu ermöglichen.

Ein Touch-Screen bietet als Instrument zur Navigation die perfekte Möglichkeit für eine Vielzahl interaktiver Multimedia Anwendungen. Viele Besucher sind mit Touch-Screens aus ihrem alltäglichen Leben, zum Beispiel bei der Verwendung von Geld- oder Fahrkartenautomaten vertraut. Touch-Screens sind robust gegen Vandalismus und können elegant in existierende Ausstellungen integriert werden.

Das letztlich im Museum Dillenburg installierte System besteht aus einem 12LCD Touch-Screen zur Interaktion und einem großformatigem Breitbild LCD Bildschirm (siehe Abb. 7). Die interaktive Anwendung wird auf einem Intel Core 2 Duo Prozessor mit 2.4 GHz und 3.5 GB Hauptspeicher und NVidia Geforce 8800 GTX Graphikchip mit 768 MB onboard Speicher betrieben.

\section{Navigation}

Die Benutzer Interaktion wurde als duales Verfahren implementiert, dass die Bedürfnisse nach einer einfachen aber dennoch vielseitigen Navigation erfüllt. Die Einfachheit des Touch-Screen ist zugleich eine der besonderen Stärken des Devices zur Benutzerinteraktion. Zur Navigation ist von dem Benutzer lediglich der Bereich von Interesse zu berühren. Für die berührten Displaykoordinaten wird ein Strahl in die Szene verfolgt und der erste Schnittpunkt mit der Geometry bestimmt, um den selektierten Bereich in der 3D-Szene zu identifizieren. Für den 


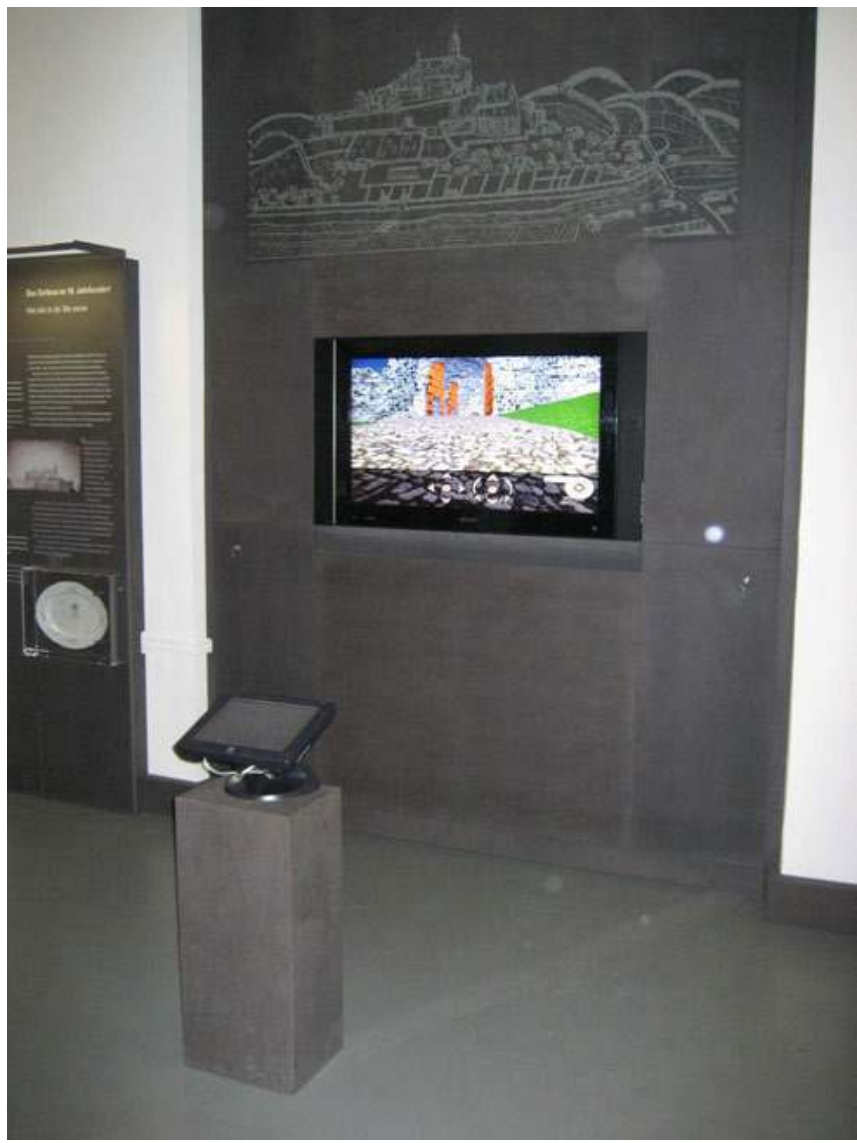

Abbildung 7: Die interaktive Installation im Museum Dillenburg. Der Touch-Screen direkt vor dem Display System dient der Navigation in der 3D-Szene.

Geometrieschnittpunkt wird in der Folge aus einer Liste im Vorwege definierter Blickpunkte derjenige ausgewählt, von dem die optimale Sicht auf den gewünschten Bereich gewährleistet ist. Die Szene wird in einem automatischen Prozess in der Form angepaßt, dass sich der Betrachter entlang eines vordefinierten Pfades von seiner aktuellen Position zu der bestimmten Betrachterposition bewegt. Diese Art der „Zeige und Bewege“ Navigation macht es insbesondere untrainierten Benutzern leicht, sich durch das virtuelle 3D Modell der Festungsanlage Dillenburg zu navigieren, ist jedoch in den Bewegungsmöglichkeiten beschränkt. Ähnlich wie bei QuickTime VR Anwendungen ist der Benutzer auf vordefinierte Blickpunkte und die Bewegungspfade zwischen ihnen eingeschränkt. ${ }^{7}$

7 Chen: QuickTime VR. 


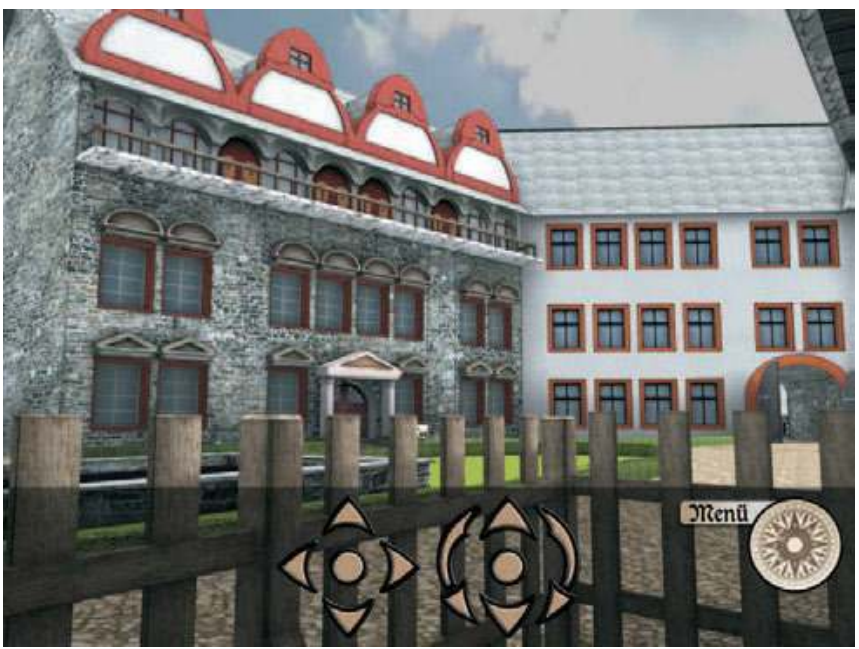

Abbildung 8: Interaktive Darstellung der 3D-Szene wie sie auf dem Touch-Screen dargestellt ist. Die GUI Elemente finden zur Steuerung der Kamera Translation und Rotation Anwendung.

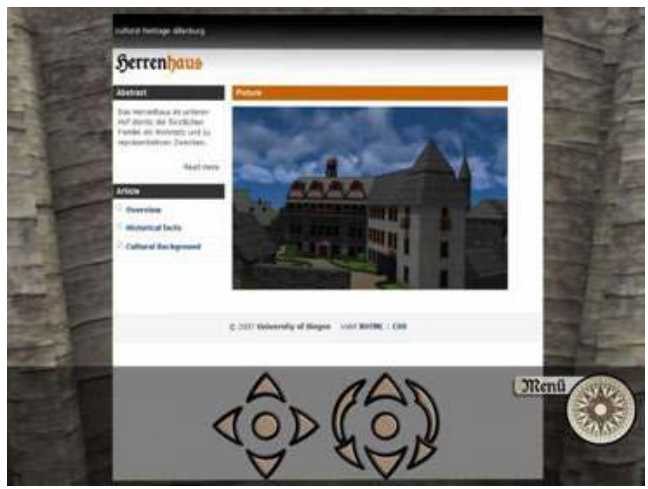

Abbildung 9: Beispiel einer virtuellen HTML basierten Informationstafel. 
Mit der Umsetzung visueller Interaktionselemente auf dem Touch-Screen zur Anpassung der lateralen und longitudinalen Bewegung der Kamera sowie des Pitch und Yaw der Kamera Blickrichtung lassen sich dem Benutzer zusätzliche Freiheitsgrade bei der Navigation einräumen. Um eine optimale Darstellung auf dem Präsentationsdisplay zu gewährleisten werden diese GUI Elemente ausschließlich auf dem Touch-Screen dargestellt (siehe Abb. 8). Diese Art der Navigation ist verwandt mit der in Spieleanwendungen zum Einsatz kommenden Navigationsmöglichkeiten und wird vor allem von technisch versierten Nutzern bevorzugt. Sie ermöglicht dem Besucher, die 3D-Szene ohne Einschränkungen zu erkunden.

\section{Zusatzmaterialien}

Mit der Bereitstellung zusätzlicher detaillierter Informationen zur Geschichte und technischen Details der Festungsanlage Dillenburg aus der interaktiven Anwendung heraus, wird die Anwendung selbst zu einem Hypermedium und avanciert zu einer virtuellen Ausstellung.

Angelehnt an traditionellen Anordnungen in realen musealen Ausstellungen, hält die interaktive Anwendung mit der Umsetzung virtueller Informationstafeln an speziellen Punkten der Szene kontextsensitive Information für den Benutzer bereit. Die virtuellen Informationstafeln gliedern sich fließend in den Gesamteindruck der virtuellen Szene ein. Die Informationen auf den Informationstafeln werden als HTML Inhalte dargestellt, die sich aus Videos, Erzählungen, Bildern und Texten zusammensetzen können. Mit der OGRE 3D Render Engine steht ein HTML Render Plugin zur Verfügung, dass die Verwendung von HTML Inhalten als Material auf polygonalen Objekten ermöglicht. Das Plugin interpretiert den HTML Inhalt und rendert die Resultate in Texturen, die in der Folge auf das polygonale Objekt angewandt werden (siehe Abb. 9). Die Inhalte der Textur werden mit jeder Änderung des HTML Inhaltes und jeglicher Benutzerinteraktion automatisch aktualisiert. Für die Interaktion werden die Benutzeranfragen direkt zur Inhaltsanpassung an das Render Plugin geleitet. Über die Verwendung des HTML Materials lassen sich so die im Vorwege offline generierten Videos auch in der interaktiven Anwendung verfügbar machen. Da die HTML Inhalte unabhängig von der interaktiven Anwendung generiert oder direkt aus dem Internet bezogen werden können, kann die Verwaltung der Zusatzinformation sehr effizient erfolgen.

\section{$6 \quad$ Zusammenfassung}

In dieser Arbeit haben wir unsere virtuelle Rekonstruktion der Festungsanlage Dillenburg, bestehend aus traditioneller computeranimierter Videos sowie der Umsetzung eines interaktiven Multimediasystems präsentiert. Seit dem 
23. Mai 2007 sind beide Komponenten fester Bestandteil der Ausstellung Festungsbau in der frühen Neuzeit im Museum Wilhelmsturm in Dillenburg.

Die Arbeiten zur virtuellen Rekonstruktion haben gezeigt, dass 3D Modelle ein wertvolles Hilfsmittel zur Interpretation und Validierung historischer Daten darstellen. Schon die initialen sehr groben 3D Modelle konnten den Historikern neue Möglichkeiten zur Dateninterpretation bieten. Die 3D Modelle waren Grundlage vieler Diskussionen bezüglich der Lage und Form der Gebäude. Daraus resultierend, konnten schon in einer frühen Modellierungsphase viele Widersprüche gelöst und Unklarheiten eindeutig geklärt werden. Mit der virtuellen Rekonstruktion wurde der direkte Vergleich verschiedener Interpretationen historischer Daten möglich und lieferte somit die verlässliche Datenbasis für die entgültige Modellierung. Von der Richtigkeit der neuen Erkenntnisse überzeugt, wurden neue Pläne zu weiteren Ausgrabungen im Areal der historischen Stätte auf Basis der virtuellen Rekonstruktion verfasst.

Für das Museum Dillenburg stellt die Multimedia Installation einen wertvollen Mehrwert für die existierende Ausstellung dar. Die Echtzeit Anwendung erlaubt dem Besucher die selbständige Erkundung der virtuellen Rekonstruktion nach eigenen Vorlieben. Die Anwendung ermöglicht weiteren Zugriff auf Hintergrundinformationen zur Geschichte der Festung und den technischen Details. Die Benutzerinteraktion wurde mit dem Ziel umgesetzt, sowohl für untrainierte als auch technisch versierte Benutzer einen angepassten Zugang zur Materie zu gewährleisten. Wir sind zuversichtlich, dass unsere Multimedia Anwendung zukünftig beide Benutzergruppen gleichermaßen motivieren wird, Interesse für die Geschichte der Festungsanlage Dillenburg zu zeigen.

\section{$7 \quad$ Danksagung}

Wir danken den Studenten der Fachgruppe Computergraphik und Multimediasysteme an der Universität Siegen für die 3D Modellierung der Festungsanlage Dillenburg und die Umsetzung der interaktiven Anwendung: Tim Horz, Matthias Hoffmann, Jan Kunze, Albert Prizkau, Michael Schantin, Jens Schlemper, Sascha Schlude, Jeong Il-Sin. Ohne die enge Kooperation mit dem Museumsverein Dillenburg e.V., insbesondere Armin Rau, Thomas Schmidt, Karlheinz Striffler, Walter Dehnert wäre dieses Projekt nicht durchführbar gewesen.

\section{Literatur}

Begand, Christian: Bricks 'n Tiles, 〈URL: www.3d-rekonstruktionen.de/ bricksntiles $\rangle$ - Zugriff am 27.04.2007.

Chen, Shenchang Eric: QuickTime VR: an image-based approach to virtual environment navigation, in: SIGGRAPH '95: Proceedings of the 22nd 
annual conference on Computer graphics and interactive techniques, New York, NY 1995, S. 29-38.

Cohen, Michael F. et al.: Wang Tiles for image and texture generation, in: ACM Trans. Graph. 22 2003, Nr. 3, S. 287-294.

Kautz, J./McCool, M.: Approximation of Glossy Reflection with Prefiltered Environment Maps, in: Proc. Graphics Interface, 2000.

Landis, H.: Production-Ready Global Illumination, in: ACM SIGGRAPH Course Notes 16, 2002.

Nister, D.: An Efficient Solution to the Five-Point Relative Pose Problem, in: IEEE Conference on Computer Vision and Pattern Recognition, 2 2003, S. 195-202.

Ogre 3D: Open Source Graphics Engine, 〈URL: www.ogre3d.org〉 - Zugriff am 27.04.2007.

Zhukov, S./Iones, A./Kronin, G.: An Ambient Light Illumination Model, in: Proc. Eurographics Rendering Workshop, 1998. 


\section{Roland Kuck/Matthias Krauß/Gerold Wesche}

\section{Prozedurale Modelle}

Virtuelle Welten können auf viele Arten erlebt werden. Eine frühe Variante, z.B. das Spiel Rogue, ${ }^{1}$ zeigte dem Spieler lediglich einfache Zeichen-basierte Grafik (siehe Abb. 1). Mit dem Fortschritt der Computergrafik und deren Anwendung in Spielen und Filmen verbindet man heutzutage mit virtuellen Welten aber immer eine realistische Darstellung. Diese benötigt noch eine Menge händischer Arbeit und ist damit zeit- und kostenintensiv. Soll die Welt dann auch noch grenzenlos wirken, ist dies auf händische Art und Weise nicht mehr zu bewältigen.

Ein Ausweg sind sogenannte prozedurale Modelle. Interessanterweise sind solche generativen Verfahren schon lange im Einsatz; so sind die Spielwelten in dem eben erwähnten Rogue nach bestimmten Regeln und unter Verwendung von Zufallszahlen erstellt und so für den Spieler jedes Mal neu. Mit steigenden Möglichkeiten der Darstellung wird die Einsatz dieser Methoden aber komplexer und es müssen unterschiedliche Verfahren für den jeweiligen Einsatzzweck entwickelt werden.

Im folgenden werden wir die verschiedenen Arten beschreiben. Schließlich werden wir uns auch mit der Frage beschäftigen, wie sich die Verfahren kombinieren und effizient für virtuelle Welten nutzen lassen.

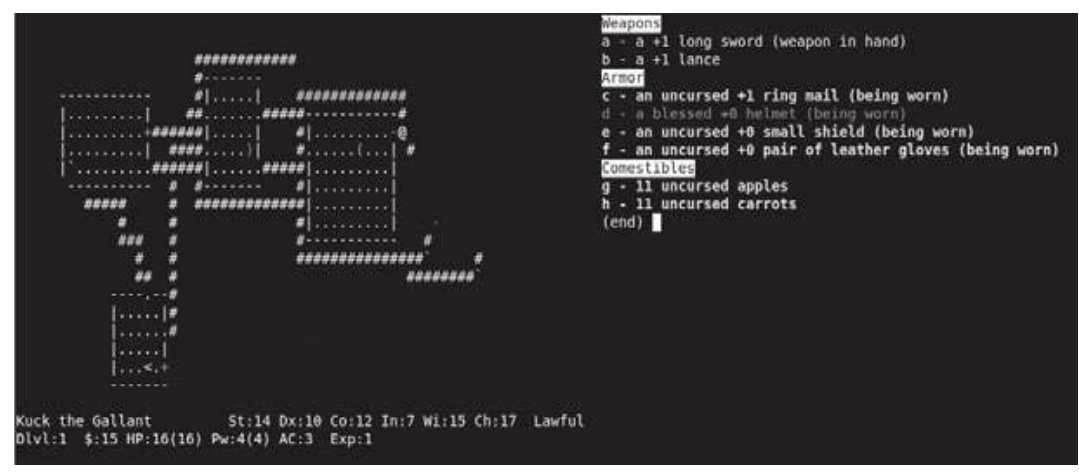

Abbildung 1: Szene des von Rogue inspirierten Spiels Nethack

1 Wikipedia: Rogue. 


\section{Definition}

Prozedurale Modelle sind Modelle, die aus einer gegebenen Prozedur und einem kleinen (Initial-)Datensatz erzeugt werden. ${ }^{2}$ Daraus ergeben sich direkt mehrere Merkmale:

- Automatische Erzengung mit keiner oder geringer manueller Arbeit. Dies resultiert vor allem aus der Anforderung an einen kleinen Datensatz. Idealerweise sollten die Daten und deren Auswirkung auf das entstehende Modell für den Bearbeiter nachvollziehbar sein, so dass das Modell gezielt angepasst werden kann.

- Wenig direkte Eingriffsmöglichkeiten für Änderungen. Dies ist die Kehrseite der automatischen Erzeugung. Notwendigerweise werden Modelle erzeugt, die nur indirekt kontrolliert werden können. Je nach Einsatzzweck gibt es verschiedene Möglichkeiten, dieses Problem in den Griff zu bekommen. Diese werden später detailierter beschrieben.

- Detailreichtum, Komplexität. Durch die gegebene Prozedur können fast beliebige Mengen an neuen Daten erzeugt werden und damit eine ebenso unbegrenzte Komplexität erzeugen.

- Geringer Speicherplatz bzw. Resourcenverbrauch zum Speichern des Modells. Der kleine Datensatz erlaubt das Erzeugen des vollständigen Modells nur genau dann, wenn es wirklich gebraucht wird. Aus GeschwindigkeitsGründen kann es dennoch notwendig sein, die prozedurale Erzeugung ganz oder in Teilen vorzuziehen und z.B. ein erzeugtes Modell im Speicher abzulegen, bzw. zu cachen.

Generell eignen sich prozedurale Modelle damit für viele Einsatzzwecke. Ideal sind sie für die Entwicklung von Prototypen. Hier kann der Eindruck durch die erhöhte Detailmenge schon in frühen Phasen besser eingeschätzt werden. Aufgrund geringer Spezifikationen sind sie aber auch einfacher anzupassen: die Stadt Köln im Mittelalter kann in die Stadt Köln heute verwandelt werden, in dem man die Regel der Generierung aus den Topographiedaten entsprechend anpasst.

Auch technisch können prozedurale Modele neue Möglichkeiten eröffnen. So kann ein bestehender Datensatz, der z.B. manuell erstellt oder automatisch erfasst worden ist, entsprechend angereichert werden, um auch unter näherer Betrachtung noch gut auszusehen. Die Darstellung und Beleuchtung kann je nach verwendeten Verfahren sogar direkt benutzt werden, um eine sowohl

2 Alvy Ray Smith bezeichnete dies als Database Amplification in Smith: Plants, fractals, and formal languages 
auf Qualität als auch auf Geschwindigkeit optimierte Darstellung zu erreichen. Beispiele sind automatisierte, gut kontrollierbare Level-of-Detail Mechanismen.

\subsection{Klassifizierung}

Prozedurale Verfahren lassen sich in nach der Art der verwendeten Prozedur einteilen. Als direktes Verfahren kann man die Verwendung einer speziellen Prozedur für einen Anwendungsfall bezeichnen. Dies wird z.B. häufig bei der Erzeugung von Pflanzen und Bäumen benutzt: In der Arbeit von Weber und Penn ${ }^{3}$ wird ein Baum in einem rekursiven Verfahren erzeugt, wobei für jede Stufe eine eigene Funktion Geometrie erzeugt. Die Grundstruktur ist somit fest vorgegeben und kann nur über eine Vielzahl spezifischer Parameter gesteuert werden. Dennoch lassen sehr verschiedene Art von Bäumen damit erzeugen. Die Anzahl der Parameter ist zwar groß, dafür ist ihre Bedeutung klar erkennbar. Konkrete Änderungen an einem Baum lassen sich so gut durchführen.

Grammatik-basierte Verfahren arbeiten mit einer abstrakten Definition der zu erstellenden Geometrie. Es müssen Ersetzungsregeln definiert werden, die ein Symbol durch eine Menge anderer Symbole ersetzt. Werden eine Menge an Regeln angewandt, enstehen eine Folge an Symbolen, die dann als Beschreibung einer Geometrie interpretiert werden kann. Dies eignet sich sehr gut für Modelle, die klar strukturiert sind, wie z.B. Pflanzen ${ }^{4}$ oder Gebäude ${ }^{5}$.

Bei der additiven Synthese werden Basisfunktionen moduliert und summiert um eine neue Funktion zu erzeugen. Dies wird vor allem für die Erzeugung von Oberflächen und Materialien, die stark strukturiert sind, wie z.B. Marmor und Holz, benutzt. Im Abschnitt 2 werden wir näher darauf eingehen.

Zur Erzeugung von Animationen von vielen einzelnen Objekten werden regelbasierte Verfahren eingesetzt. Ein einzelnes Objekt erhält einen Satz Regeln, der beschreibt, wie es sich aufgrund seiner Sinneseindrücke verhalten soll. Durch eine Simulation kann dann für eine Vielzahl von Objekten ein stimmiges Gesamtresultat berechnet werden.

\section{$2 \quad$ Umsetzung und Anwendbarkeit}

Als Beispiel, wie die oben skizzierten Verfahren umgesetzt und angewendet werden können, gehen wir im Folgenden näher auf die additive Synthese ein.

3 Weber/Penn: Creation and rendering of realistic trees.

4 Prusinkiewicz/Lindenmayer: The alogrithmic beauty of plants.

5 Müller et al.: Procedural Modeling of Buildings 


\subsection{Basisfunktionen und Synthese}

Zur Synthese wird eine kleine Menge an Basisfunktionen benötigt. Diese unterscheidet sich nach Art der Anwendung. Soll z.B. ein Helligkeitswert für jeden Punkt im Raum ermittelt werden, z.B. um einen massiven Körper darzustellen, wird eine Funktion der folgenden Art benötigt:

$$
f: \mathbb{R}^{3} \rightarrow \mathbb{R}
$$

Diese Funktion wird zur Synthese nun im Wertebereich skaliert, d.h. gestreckt oder gestaucht, und gewichtet addiert:

$$
s(x)=\sum_{k=0}^{n} \alpha_{k} f\left(\beta_{k} x\right),
$$

mit $\alpha_{k}$ als Gewichtungsfaktor und $\beta_{k}$ als Frequenz der $k$ Funktionen.

Basisfunktionen müssen mehrere Eigenschaften erfüllen um sinnvoll einsetzbar zu sein:

- $\quad$ Eine Basisfunktion muss wiederholbar sein, d.h. gleiche Eingabewerte müssen die gleichen Ausgabewerte erzeugen. Dies ist notwendig, um beim Berechnen mehrerer Bilder, wie dies u.a. bei der Echtzeit-Computergrafik der Fall ist, immer das gleiche Modell darstellen zu können.

- $\quad$ Die Funktion muss Bandbreiten-limitiert sein, idealerweise normiert, d.h. mit einer Maximalfrequenz von 1. Die Notwendigkeit dieser Eigenschaft für die Darstellung wird in Abschnitt 2.2 näher erklärt, hier sei nur darauf hingewiesen, dass durch die Begrenzung der Maximalfrequenz eine Skalierung im Definitionsbereich erst möglich ist und dies für das Formen von Mustern eine wichtige Voraussetzung ist.

- Analog zur Maximalfrequenz muss auch der Wertebereich beschränkt sein, idealerweise wieder in einem normierten Bereich, etwa von $[0,1]$ oder $[-1,1]$.

Diese Eigenschaften werden von vielen Funktionen erfüllt, wie z.B. der Sinus-Funktion. In der Tat lassen sich sämtliche andere Funktionen durch Summation von gewichteten Sinus-Basisfunktion verschiedener Frequenzen darstellen.

Interessanter sind aber Basisfunktionen, die mit Hilfe von (Pseudo-)Zufallszahlen funktionieren, häufig auch Noise $^{6}$ genannt werden. Die Noise-Funktion

6 engl. für Rauschen 

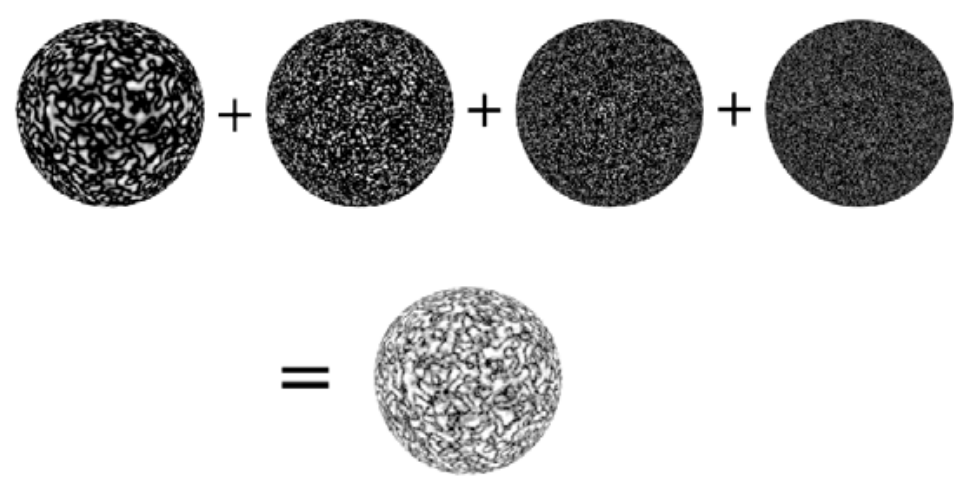

Abbildung 2: Mehrere Noise-Funktionen mit unterschiedlicher Maximalfrequenz ergeben addiert eine granitartige Struktur

von Perlin ${ }^{7}$ ist dabei einer der bekanntesten Vertreter und ist darüber hinaus relativ schnell zu berechnen ${ }^{8}$. In Abb. 2 sieht man ein einfaches Beispiel dazu.

\subsection{Adaptive Komplexität}

Zur Generierung von Bilder in der Computergrafik werden heutzutage eigentlich immer Rasterisierungverfahren eingesetzt, die die Farbwerte einzelner weniger Lichtstrahlen ermitteln. Bei diesen Verfahren ist es notwendig, dass Modelle nicht zu viele Details enthalten, weil ansonsten sogenannte Aliasing-Artefakte auftreten. Dies äussert sich z.B. bei Animationen in einem flackernden Rauschen. Je besser ein Modell diese Details adaptiv zur Verfügung stellen kann, desto weniger muss auf aufwendige und rechenintensive Gegenmassnahmen, sogenanntes Anti-Aliasing, zurückgegriffen werden.

Die additive Synthese bietet hierfür eine sehr einfache und effektive Lösung. Die einzelnen Funktionen haben als Voraussetzung eine Maximalfrequenz, d.h. eine maximale Menge an Details. Bei der Berechnung des Farbwertes kann nun eine je nach Blickpunkt auf das Modell maximal darstellbare Frequenz berechnet werden und alle Funktionen bei der Synthese mit einer höheren Frequenz nicht verwenden. Normalerweise wird dies nicht mit einem harten Schwellwert sondern einem Übergangsbereich realisiert, in dem das Gewicht der Funktion schrittweise reduziert wird.

7 Perlin: An Image Synthesizer.

8 Ein Verbesserung des Verfahrens wurde von Perlin selber in Perlin: Improving Noise beschrieben. Andere Verfahren finden sich z.B. in Ebert et al.: Texturing and Modeling 
Diese einfache Massnahme erhöht nicht nur die visuelle Qualität, sie ist auch ein einfaches Level-of-Detail-Verfahren, welches immer weniger Details berechnet und damit weniger Rechenzeit verbraucht, je weiter sich der Betrachter von dem Modell entfernt.

\subsection{Reduzierung von Laufzeitkosten}

Die adaptive Synthese aus dem vorherigen Abschnitt sorgt dafür, dass der Rechenaufwand an die darzustellende Komplexität gekoppelt ist. Die Laufzeit kann aber auch beliebig gross werden, wenn entsprechend viele Details dargestellt werden sollen, z.B. weil der Betrachter sehr nahe am Modell steht.

Hinzu kommt, dass die Berechnung der Basisfunktionen an sich auch höhere Laufzeitkosten verursachen, als z.B. ein einfacher Zugriff auf eine Textur ${ }^{9}$ und die Daten immer wieder neu berechnet werden: So verändern sich Modelle oft nur allmählich über die Zeit. Eine Wand aus Marmor hat z.B. immer das gleiche Muster und auch der Blickwinkel oder Abstand wird sich nicht viel verändern, wenn der Betrachter längere Zeit vor einer Skulptur steht und die Wand nur im Hintergrund sichtbar ist.

Die einfachste Reduzierung der Rechenleistung ist auf Kosten des Speicherbedarfs der Anwendung möglich und besteht darin, das Modell komplett vorzuberechnen. Dies wird baking ${ }^{10}$ genannt. Bei einem prozeduralen Oberflächenmodell wird ein passendes Bild berechnet und als Textur auf die Geometrie aufgebracht.

Durch die festgelegte Auflösung des Bildes geht einer der Vorteile der prozeduralen Modelle verloren: Der Betrachter sieht ab einem Punkt keine weiteren Details mehr. Gemildert werden kann dies durch Detail-Texturen. Hierbei werden ähnlich der Basisfunktionen mehrere Texturen summiert, wobei die Detail-Textur erst von nahen sichtbar ist und kachelbar ist, d.h. nahtlos aneinander gelegt werden kann.

Sollen echte Details sichtbar sein, muss eine entsprechend grosse Textur verwendet werden. Sogenannte Clipmaps $^{11}$ sind ein Verfahren, das es erlaubt grosse Texturen zu verwenden, die nur in Teilen im Speicher der Grafikkarte vorliegen müssen, um dargestellt werden zu können. Bei geringen Veränderungen des Blickpunktes ergeben sich hierbei auch nur geringe Änderungen an der Untermenge der Bilddaten, so dass bei kontinuierlichen Bewegungen der zusätzliche Rechenaufwand minimal ist.

9 Insgesamt steigt die Rechenleistung schneller als die zur Verfügung stehende Speicherbandbreite. Von daher reduzieren sich die realen Kosten der Berechnung immer mehr im Vergleich zum einfachen Texturzugriff.

10 engl. für backen

11 Tanner/Migdal/Jones: The clipmap: a virtual mipmap. 
Anstelle die Daten aus dem Hauptspeicher bzw. von Festplatte nachzuladen, können die Daten aber auch bei Bedarf erst erzeugt werden. Ein solches hybrides Verfahren vereint die Vorteile der prozeduralen Verfahren, in dem (fast) beliebig viele Details sichtbar sind, zugleich aber Daten nicht für jedes Bild erneut berechnet werden müssen. Auch wird lediglich ein Ausschnitt der Daten benötigt, d.h. der zusätzliche Speicherbedarf ist moderat.

Die bisher beschrieben Verfahren lassen sich nicht nur auf Farbwerte anwenden. Speziell Clipmaps können z.B. auch gut benutzt werden, um aus einem Bild, welches Höhenwerte anstelle von Farbwerten speichert, eine Höhenkarte zur Laufzeit zu erstellen. ${ }^{12}$

\subsection{Komposition}

Die oben angesprochenen Detail-Texturen sind ein Beispiel für die allgemeinere Technik der Komposition. Ähnlich der Synthese von Basisfunktionen können verschiedene Verfahren kombiniert werden, um so ein optimiertes Gesamtergebnis zu erhalten. Dies ist vor allem deswegen interessant, weil auf diese Art und Weise auch ein Künstler in den Prozess einwirken kann und so ein für ein bestimmten Anwendungszweck optimiertes Ergebnis enstehen kann.

Auch wenn der Art der Kombination eigentlich keine Grenzen gesetzt sind, werden wir uns im folgenden auf eine Untermenge beschränken, in denen sich die zu kombinierenden Funktionen als eine Abbildung eines Punktes im Raum auf einen Farbwert ansehen lassen. Den Farbwert kann als Zahlentupel definiert werden, welcher - wie in der Computergrafik üblich - die Mischanteile von Rot, Grün und Blau angibt. Zusätzlich wird ein sogenannter $A \not p h a-W e r t^{13}$ definiert, der wie die Farbwerte in einem normierten Wertebereich von $[0,1]$ liegt und den Anteil der Verdeckung angibt.

Die Eingabefunktionen können sowohl prozedural erzeugt sein, als auch z.B. in Form von Bild- bzw. Texturdaten von einem Künstler eingebracht werden. In Abb. 3 ist dafür ein Beispiel zu sehen.

Es gibt verschiedene Operatoren, die diese Eingabefunktionen zur einer neuen verknüpfen:

- $\quad$ Stellen die Eingaben mehrere Ebenen eines Materials dar, können sie mittels des sogenannten Over-Operators visuell realistisch übereinander gelegt werden.

12 Siehe Losasso/Hoppe: Geometry clipmaps: Terrain rendering using nested regular grids und Asirvatham/Hoppe: Terrain rendering using GPU-based geometry clipmaps. Auch hierbei wird eine (prozedural) erzeugte Detail-Textur benutzt werden, um den Realismus zu erhöhen.

13 Eingeführt in Porter/Duff: Compositing digital images. In dieser Arbeit ist auch der oben beschriebenen Over-Operatoren der Compositing Algebra definiert. 

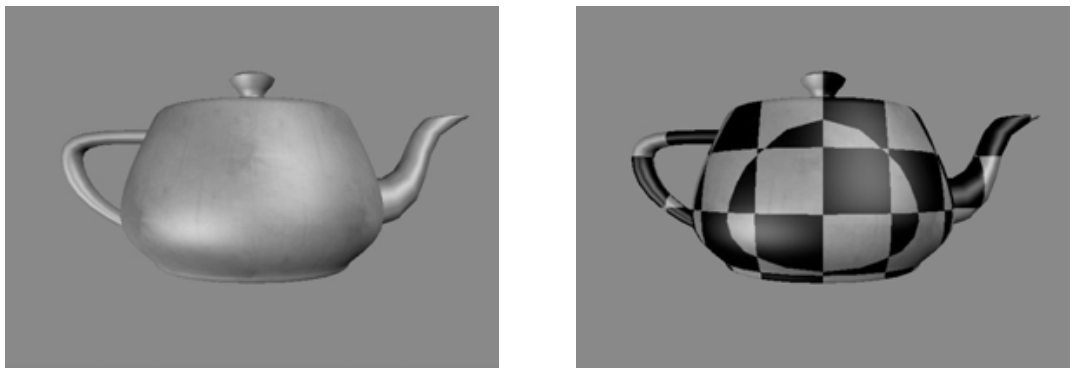

Abbildung 3: Das linke Bild zeigt eine texturierte Teekanne. In dem rechten Bild wurde eine Schachbrettmuster auf die Teekanne angewendet, wobei die weißen Flächen durch die Textur des linken Bildes ersetzt wurde.

- $\quad$ Ein kontinuierlicher Wechsel zwischen mehreren Materialien kann durch eine weitere (Selektions-)Funktion beschrieben werden. So kann z.B. eine abstrakte, manuell erstellte Karte angeben, wo Grass, Sand und Wasser sind und an entsprechenden die jeweilige Eingabefunktionen ausgewählt werden.

- $\quad$ Als Hilfsoperatoren ist es nützlich Funktionen skalieren und mit logischen Operatoren verknüpfen zu können, z.B. um die Selektionsfunktion aus anderen Eingaben herzuleiten.

\section{Ganzheitliche Prozesse}

Der letzte Abschnitt zeigte bereits, dass die Kombination von mehreren Verfahren eine Vereinfachung der Handhabbarkeit, sowie einer weitere Steigerung der visuellen Komplexität und Qualität mit sich bringt.

Von daher erscheint es sinnvoll, die Frage nach einem ganzheitlichen Prozess zu stellen, der prozedurale Verfahren sowie manuell erstellte Modelle aller Art erfasst und direkt kombinierbar macht. Er sollte folgende Eigenschaften besitzen:

- Modelle sollen kombinierbar sein, wobei der Prozess die Anordnung der Modelle zueinander unterstützen sollte. Hierbei sind Informationen über Einschränkungen und Hilfestellungen notwendig, die ein Modell als Metadaten enthalten muss.

- $\quad$ Da eine globale Sicht aller Daten und Verknüpfungen vorliegt, kann eine Realisierung des Gesamtmodells an einen speziellen Kontext angepasst werden. So kann auf Geräten mit geringer Leistung, wie z.B. auf einem Mobiltelefon, das Modell automatisch in geringere Komplexität erstellt werden. Dies schließt sowohl die Reduzierung einzelner Komponenten, aber auch das Weglassen oder Ersetzen von Komponenten ein. 
- Die Hilfestellung bei der Erstellung kann weiter fortgeführt werden, indem der Prozess nicht nur bei der Kombination, sondern auch schon bei der Auswahl der einzelnen Modelle hilft, z.B. in dem in entsprechenden Bibliotheken gesucht werden kann.

- Die vorhandenen Strukturen können genutzt werden, um eine semantischen Informationen abzuleiten, die dann von der Anwendung verwendet werden können.

Solch ein Prozess hat für alle beteiligten Personen Vorteile:

- $\quad$ Die Autoren von Modellen profitieren von einem breiteren Publikum, da die Modelle besser wiederverwendbar sind.

- Anwendungsentwickler können sich mehr auf den Inhalt und weniger auf die notwendige Technologie konzentrieren. Die erlaubt vor allem die Einbindung von Personen mit geringen Technik-Know-how in den Entwicklungsprozess.

- $\quad$ Die Benutzer profitieren von besseren und vielfältigeren virtuellen Welten.

Ein Beispiel kann dieses Vorgehen verdeutlichen: Ein Spielautor möchte eine Szene mit einer Hütte im Wald in einer typischen hügeligen mitteleuropäischen Landschaft erstellen. Dazu modelliert er spielrelevante Aspekte in der Hütte mit traditionellen Mitteln. Zudem gibt er in seinen Basisdaten die grundlegenden Daten in maschinenlesbarer Form an: Hügelige Landschaft, geographische Lage, die Hütte, ein Weg.

Der Steuerungsprozess der Geometrieerzeugung verfügt über ein Portfolio von Generatoren und über einen Regelsatz, der allgemeines, szenenunabhängiges Wissen über räumliche Eigenschaften der Welt enthält. Aufgrund der Basisdaten wählt der Prozess einen Hügelgenerator, der die Grundfläche der Szene erstellt und mit Metadaten über die Grundbeschaffenheit versieht, die den nachfolgenden Schritten helfen.

Das Grundwissen über allgemeine Beschaffenheit von Welten enthält beispielsweise Wissen darüber, dass auf Wegen und in der unmittelbaren Umgebung von Gebäuden normalerweise keine Bäume stehen. So kann er die entsprechenden Bereiche für Vegetation sperren.

Der Steuerprozess wählt im Folgenden einen Vegetationsgenerator, der Pflanzen und weitere Objekte wie z.B. Steine oder Flüsse in der Szene verteilt. Die Metadaten des Landschaftsgenerators helfen nun, geeignete Pflanzentypen aus einem Repertoire zu finden. Die Pflanzen selbst werden wiederum von spezialisierten Generatoren erzeugt.

Auf diese Weise können Umgebungen, weitläufige Landschaften und ähnliches automatisch aber kontrolliert erzeugt werden. Trotzdem bleiben die 
Basisdaten übersichtlich und verständlich. Der Autor kann seine Angaben nach ersten Durchläufen verfeinern, um das gewünschte Ergebnis zu erhalten.

Dieser Prozess schafft bereits für eine einzige Szene erhebliche Arbeitserleichterungen. Aber darüber hinaus können etliche Szenenvariationen geschaffen werden, beispielsweise um spezifische Eigenheiten und Beschränkungen verschiedener Geräteklassen zu berücksichtigen. Der Regelsatz kann diese Eigenheiten besser berücksichtigen, wenn der Autor Prioritäten in seinen Grunddaten angibt. So kann z.B. verhindert werden, dass der Generator bei einer Szene für beschränkte Mobilgeräte wichtige Aspekte der Szene ausspart.

\section{$4 \quad$ Fazit}

Prozedurale Modelle werden heutzutage bereits vielseitig eingesetzt. Die steigende Grafikkartenleistung wird ihren Einsatz auch im Echtzeit-Bereich und somit für virtuelle Welten weiter voranbringen. Dennoch wird es immer notwendig sein, dass ein Künstler Anpassungen vornehmen kann, und in einer geeigneten Kombination aller Verfahren lässt sich dies, auch schrittweise, realisieren.

Der skizierte ganzheitliche Prozess kann hierbei helfen, zuerst muss er aber konkretisiert und auf seine Zielsetzung hin überprüft werden. Der Prozess kann dann in der Rolle einer Basistechnologie für virtuelle Welten das Erstellen und Anpassen dieser deutlich vereinfachen und somit dazu beitragen, dass virtuelle Welten selber als Basistechnologie angesehen werden können, um Wissen und Erfahrungen zu vermitteln.

\section{Literatur}

Asirvatham, Arul/Hoppe, Hugues: Terrain rendering using GPU-based geometry clipmaps, in: GPU Gems 2, Reading, CA 2005, S. 27-45.

Ebert, David S. et al.: Texturing and Modeling: A Procedural Approach, San Francisco, CA 2002.

Losasso, Frank/Hoppe, Hugues: Geometry clipmaps: Terrain rendering using nested regular grids, in: SIGGRAPH 2004, New York, NY 2004, S. 769-776.

Müller, Pascal et al.: Procedural Modeling of Buildings, in: SIGGRAPH 2006, New York, NY 2006, S. 614-623.

Perlin, Ken: An Image Synthesizer, in: SIGGRAPH 85, New York, NY 1985, S. 287-296.

Perlin, Ken: Improving Noise, in: SIGGRAPH 2002, New York, NY 2002, S. 681-682. 
Porter, Thomas/Duff, Tom: Compositing digital images, in: SIGGRAPH '84, New York, NY 1984, S. 253-259.

Prusinkiewicz, Przemyslaw/Lindenmayer, Aristid: The alogrithmic beauty of plants, New York, NY 1991/1996.

Smith, Alvy Ray: Plants, fractals, and formal languages, in: SIGGRAPH '84, New York, NY 1984, S. 1-10.

Tanner, Christopher C./Migdal, Christopher J./Jones, Michael T.: The clipmap: a virtual mipmap, in: SIGGRAPH 98, New York, NY 1998, S.151-158.

Weber, Jason/Penn, Joseph: Creation and rendering of realistic trees, in: SIGGRAPH 95, New York, NY 1995, S. 119-128.

Wikipedia: Rogue, 〈URL:http://de.wikipedia.org/wiki/Rogue_ (Computerspiel) $)$ - Zugriff am 06.12.2008. 



\section{Autoren}

Doris Agotai, Dr. sc. ETH, dipl. Arch. ETH, ist wissenschaftliche Mitarbeiterin im Forschungsbereich „Virtual Environments“ am Institut für 4D-Technologien (FHNW). Sie lehrt an der ETH Zürich, an der Zürcher Hochschule der Künste (ZhdK) und an der Hochschule Liechtenstein und ist Autorin von „Architekturen in Zelluloid. Der filmische Blick auf den Raum“ (Bielefeld 2007).

Steffi Beckhaus, Prof. Dr.-Ing., ist Professorin für Informatik an der Universität Hamburg. Sie leitet die im.ve Arbeitsgruppe, die das Thema ,interactive experiences“ aus der Perspektive der Virtual Reality, der Computergrafik und der Medienkunst bearbeitet. Ihre Forschungsinteressen sind unter anderem die menschliche Wahrnehmung, die Navigation und Interaktion in virtueller Realität und menschzentrierte, intuitive, reichhaltige Computerschnittstellen.

Manfred Bogen, Ph.D., ist Abteilungsleiter „Virtual Environments“ am Fraunhofer-Institut für Intelligente Analyse- und Informationssysteme IAIS. Sein primärer Forschungsschwerpunkt ist die Ermöglichung von Kommunikation und Kooperation. Dieser Schwerpunkt wandelte sich von Rechnerkopplung und Electronic Mail in den frühen Jahren über Multimedialen Diensten mit und ohne World Wide Web zur Virtual und Augmented Reality in Virtual Environments heute.

Mario Doulis, Prof., Dipl.-Des. FH, ist Professor für Interface Design an der Hochschule für Technik FHNW und Professor für Neue Medien an der Merz Akademie Stuttgart. Er leitet den Forschungsbereich „Virtual Environments“ am Institut für 4D-Technologien i4Ds der FHNW, forscht zum Thema Interface Design mit Schwerpunkt Spatial Interface und ist Mitglied in mehreren Gremien im Bereich 3D User Interface. Mario Doulis lehrt in den Gebieten Informationsvisualisierung und User Interface Design.

Tim Jansen, Dipl.-Ing. Medientechnik, arbeitet freiberuflich im Bereich der Entwicklung von 2D und 3D User Interfaces für interaktive Installationen, Rich Internet Applications und mobile Anwendungen. Im Kontext seiner Diplomarbeit stand die kollaborative Interaktion in virtuellen Umgebungen und die Entwicklung eines 3D User Interfaces im Mittelpunkt.

Andreas Kolb ist seit Ende 2003 Leiter des Lehrstuhls für Computergraphik und Multimediasysteme der Universität Siegen. Sein akademischer Hintergrund ist Computergraphik, Visualisierung und Virtuelle Realität. Er hat seine Doktorarbeit 1995 an der Universität Erlangen unter der Betreuung von Prof. Hans-Peter Seidel durchgeführt. Andreas Kolb ist Mitglied der ACM, der GI, der EG, 
sowie der universitären Forschungszentrums für Sensorsystem (ZESS) an der Universität Siegen. Seine Forschunginteressen umfassen Computergraphik und Computer Vision, insbesondere die Visualisierung wissenschaftlicher Daten und Sensordaten, der Echtzeitverarbeitung von Sensordaten, bildbasiertes Rendering und Virtuelle Realität.

Matthias Krauß, Dipl. Inform., hat Informatik an der Uni Hildesheim und Uni Bremen studiert. Zwischen 2002 und 2006 arbeitete er als wissenschaftlicher Mitarbeiter im internationalen, hochschulübergreifenden Studiengang Digitale Medien in Bremen. Seit 2006 arbeitet er am Fraunhofer-Institut für Intelligente Analyse- und Informationssysteme IAIS. Seine Forschungsinteressen beinhalten Visualisierung, Interface- und Interaktionsdesign sowie Computergrafik.

Rolf Kruse gründete - nach dem Architektur-Studium und lehrreichen Jahren bei „Art + Com“ in Berlin - 1997 das „Labor für Mediale Architekturen“ für die Entwicklung innovativer räumlicher Medienschnittstellen. 2002 wurde ihm vom Fraunhofer IGD der Aufbau und die Weiterentwicklung des Cybernarium als geschäftsführender Creative Director übertragen. Seit 2005 realisiert er als Geschäftsführer der Invirt GmbH mit einem interdisziplinären Team mediale Anwendungen, die komplexe wissenschaftliche und kulturelle Themen für ein breites Publikum erlebbar machen. Neben der Veröffentlichung von Artikeln in Fachbüchern vermittelt er sein Wissen seit 2006 an Studenten der Hochschule Darmstadt (seit 2008 als Vertretungsprofessor).

Roland Kuck, Dipl.-Inform., arbeitet beim Fraunhofer-Institut für Intelligente Analyse- und Informationssysteme IAIS. Seine Forschungsschwerpunkt sind Verfahren und Systeme zur Echtzeit-Darstellung von komplexen Materialien und Körpern.

Christof Rezk-Salama ist wissenschaftlicher Assistent am Lehrstuhl für Computergraphik und Multimediasysteme der Universität Siegen. Er hat an der Universität Erlangen-Nürnberg Informatik studiert und 2002 als Stipendiat im DFG-Graduiertenkollegs „3D Bildanalyse und Synthese“ mit Auszeichnung promoviert. Christof Rezk Salama hält regelmäßig Vorlesungen, Kurse und Seminare über Computergraphik, wissenschaftliche Visualisierung, Charakteranimation und Graphikprogrammierung. Er hat praktische Erfahrung in unterschiedlichen Anwendungsgebieten der Computergraphik, darunter Medizin, Geologie und Archäologie. Gemeinsam mit internationalen Coauthoren hat er das Buch „Real-Time Volume Graphics“ (AKPeters, 2006) über die Echtzeitdarstellung von Volumendaten (z.B. Tomographieaufnahmen aus der Medizin) veröffentlicht.

Jens Schröter, Prof. Dr., lehrt „Theorie und Praxis multimedialer Systeme“in der Medienwissenschaft der Universität Siegen. Seine Forschungsschwerpunkte 
sind Theorie und Geschichte digitaler Medien, Theorie und Geschichte der Fotografie, Dreidimensionale Bilder, Intermedialität

Christian Spies, Dr. phil, ist Assistent am kunsthistorischen Seminar der Universität Basel und am nationalen Forschungsschwerpunkt Bildkritik, eikones. Seine Arbeitsschwerpunkte sind Bildtheorie und -geschichte der Moderne, Bilderstreite der Moderne, monochrome Malerei und Künstlervideo. Jüngste Veröffentlichungen sind Die Trägheit des Bildes. Bildlichkeit und Malerei zwischen Malerei und Künstlervideo, München 2007, Movens Bild. Zwischen Evidenz und Affekt, München 2008 (gemeinsam mit Gottfried Boehm und Birgit Mersmann).

Severin Todt hat sein Studium an der Fachhochschule erfolgreich mit dem Titel Diplom Medieninformatik(FH) abgeschlossen und im postgradualen Studiengang an der Fachhochschule Wedel den Titel Master of Science erworben. An der Universität Siegen hat er mit dem Titel Diplom Informatiker das Studium der Angewnadten Informatik abgeschlossen. Seit Oktober 2003 arbeitet er als wissenschaftlicher Mitarbeiter am Lehrstuhl für Computergraphik an der Universität Siegen. Er hält dort regelmäßig Vorlesungen und Seminare zu den Themen Computergraphik, 3D Modellierung, Charakter Animation und Graphikprogrammierung. Derzeit schließt er seine Doktorarbeit zu dem Thema „Real-Time Acquisition and Rendering of Spherical Light Fields“ ab. Seine Forschungsinteressen umfassen bildbasierte Rendering Technologien, Virtual Reality Technologien und deren Einbindung in industrielle Prozesse sowie Mensch-Maschine Interaktion.

Markus Wacker ist Professor für Computergraphik an der Hochschule für Technik und Wirtschaft (FH) Dresden. Seine Forschungsschwerpunkte sind Virtual Reality mit Navigation, Interaktion und Simulation, Gestenbasierte Interfaces (Multitouch), technische Modellierung und Visualisierung. Umgesetzte und laufende Projekte: Integrative Produktentwicklung virtueller Prototypen (INPROVY), Navigations- und Interaktionskonzepte in einer Cave, Entwicklung eines Multitouch-Tischs und neuartige Anwendungen von Mehrfingergesten, Visualisierung des Dresdner Zwingers und des Nymphenbads, Output4business: Verknüpfung von Wissenschaft und Wirtschaft.

Cornelius Weidner schloß 2000 bei CSC-Ploenzke seine Ausbildung zum Anwendungsentwickler ab und war bis 2002 dort Angestellter als Junior Professional Consultant. Mit sich steigerndem Interesse an digitalen Medien wechselte er 2002 zum neuen englischsprachigen Studiengang Media Production an der Hochschule Darmstadt, vollzog ein Jahr davon im Auslandsstudium an der Escuela Politechnica Superior Valencia im Fach Communicación Audiovisual, und vertiefte sein Interesse an Virtuellen Realitäten mit einem Praktikum am 
Cybernarium Darmstadt und der Abschlußarbeit zur Begehung von Virtuellen Realitäten zum B.A. in Media Production. Seit 2008 ist er Angestellter der Invirt GmbH, welche sich intensiv der Nutzung Virtueller Realitäten und deren Schnittstellen zum Menschen widmet.

Gerold Wesche, Dr., arbeitet als Senior Researcher am Fraunhofer-Institut für Intelligente Analyse- und Informationssysteme IAIS. Seine Arbeitsgebiete umfassen Virtual Environments, Visualisierung, 3D-User-Interfaces, geometrische Modellierung und Kurven und Flächen des Computer-Aided-Design. Er ist Autor und Ko-Autor zahlreicher Publikationen in wissenschaftlichen Journalen und für internationale Konferenzen, einschließlich ACM VRST, IEEE Virtual Reality und Eurographics Workshop on Virtual Environments. Er hat an der Universität des Saarlandes über Modellierung von Freiformflächen in virtuellen Umgebungen promoviert.

Gundolf Winter, Prof. Dr., lehrt Kunstgeschichte an der Universität Siegen. Er ist Projektleiter B7 „Virtualisierung von Skulptur“ am Forschungskolleg „Medienumbrüche“. Seine Forschungsschwerpunkte sind Bildkonzepte der Moderne, Medientheorie der Skulptur und der Architektur.

Hans Peter Wyss, Dipl. Künstler HGK, Dipl. Multimedia Producer SAE, ist Forschungsassistent im Bereich „Virtual Environments“ am Institut für 4DTechnologien (FHNW). Er forscht zum Thema Interaction- und Interface Design für virtuelle Umgebungen und beschäftigt sich mit Aspekten interaktiver Medienkunst. 